\begin{abstract}
Title of Thesis:

Thesis Directed By:

\author{
MICRO AIR VEHICLE SCALE GUST-WING \\ INTERACTION IN A WIND TUNNEL
}

Zachary F. Smith, Master of Science, 2018

Associate Professor Anya Jones

Department of Aerospace Engineering
\end{abstract}

Studying isolated gust effects on simple airfoil models in a controlled environment is a necessity to further the development of MAV gust response and control laws. This work describes the creation of a vertical gust generator in a low speed, low turbulence wind tunnel through the use of an actuated fan placed below the tunnel and ducted through its floor. Gusts of up to $40 \%$ of the freestream velocity were created. Characterization of the gust generator is shown, and its interaction with a stationary wing at several angles of attack is evaluated. The actuated gust profile is also compared to that of a pitched wing in a gust-less environment with many visible similarities. 


\title{
MICRO AIR VEHICLE SCALE GUST-WING INTERACTION IN A WIND TUNNEL
}

\author{
by \\ Zachary F. Smith \\ Thesis submitted to the Faculty of the Graduate School of the \\ University of Maryland, College Park, in partial fulfillment \\ of the requirements for the degree of \\ Master of Science \\ 2018
}

Advisory Committee:

Associate Professor Anya R. Jones, Chair

Professor James Baeder

Assistant Professor Stuart J. Laurence 
(C) Copyright by

Zachary F. Smith 2018 


\section{Acknowledgements}

I would like to thank Dr. Jones and Dr. Hrynuk for giving me this opportunity to pursue research co-sponsored by the University of Maryland and the Army Research Laboratory. Their guidance throughout this project was immeasurable. I would also like to extend a thanks to Howard Carpenter and Jeffery Westrich at ARL for their design insight and manufacturing support that helped to make the gust generator a reality. And to Chris Kroninger for his review of my reports and presentations as well as for helping me obtain funding through ORAU and MAST-CTA.

I must thank everyone that has shared in my journey at the University of Maryland both inside and outside of STAL. Special thanks to Drs. Field, Gino, Peter, Andrew, and Andre for being a source of insight from people who have "been there". And to Jonathan and Hulya for helping me survive classes and keeping me sane. And a final thanks to my family and friends outside of the University for their support. With a special thanks to my fiancé, Caitlyn.

Research was sponsored by the Army Research Laboratory and was accomplished under Cooperative Agreement Number W9911NF-16-2-0176. The views and conclusions in this document are those of the authors and should not be interpreted as representing the official policies, either expressed or implied, of the Army Research Laboratory or the U.S. Government. The U.S. Government is authorized to reproduce and distribute reprints for Government purposes notwithstanding any copyright notation herein. This research was also supported by the Micro Autonomous Systems and Technology (MAST) Collaborative Technology Alliance (CTA) under cooperative agreement number W911 NF-08-2-0004. 


\section{Table of Contents}

Acknowledgements ............................................................................. ii

Table of Contents ................................................................................. ii

List of Tables .........................................................................................

List of Figures ..................................................................................... vi

List of Abbreviations ..............................................................................

Chapter 1: Introduction ........................................................................... 1

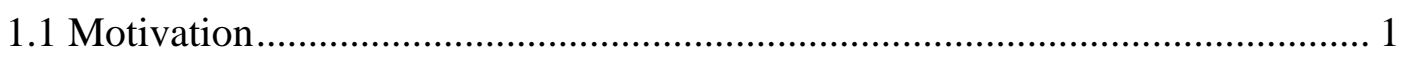

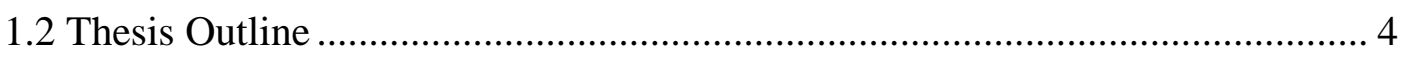

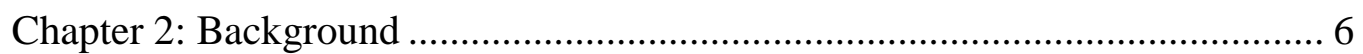

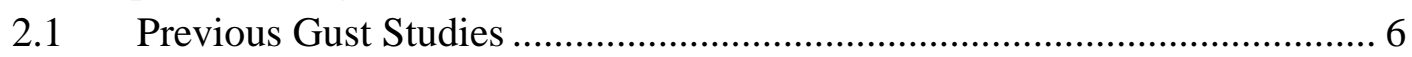

2.1.1 Full Scale and Outdoor Testing ........................................................... 6

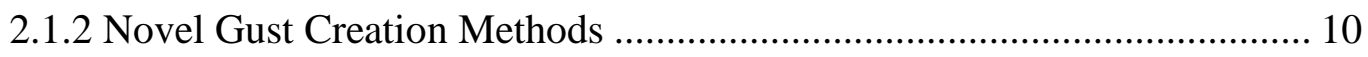

2.1.3 Oscillating Vanes and Shutter Gust Generators...................................... 17

2.1.4 Plunging and Pitching Wings to Mimic Gusts........................................ 23

2.1.5 Vertical Gust Generators in Water Tunnels and Tow Tanks.................... 26

2.1.6 Computational Methods for Gusts .......................................................... 30

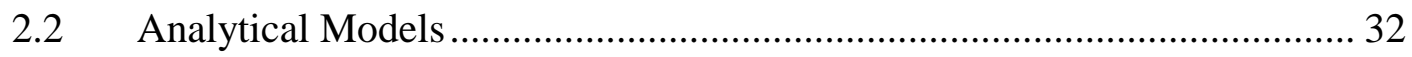

2.3 Scope of Present Research ................................................................... 34

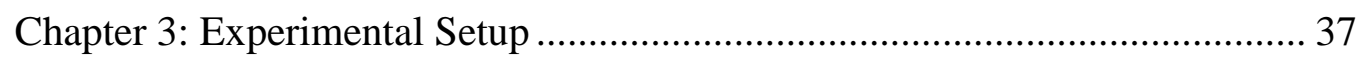

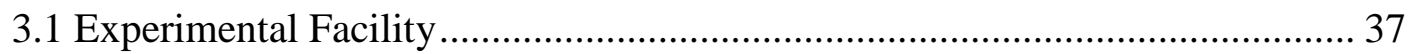

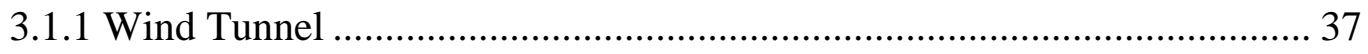

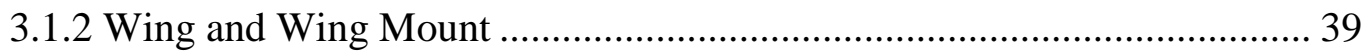

3.2 Gust Design......................................................................................... 40

3.2.1 Gust Design Goals .................................................................... 40

3.2.2 Fan Research and Selection ............................................................. 41

3.2.3 Final Design ................................................................................... 44

3.3 PIV: Particle Image Velocimetry ........................................................... 47

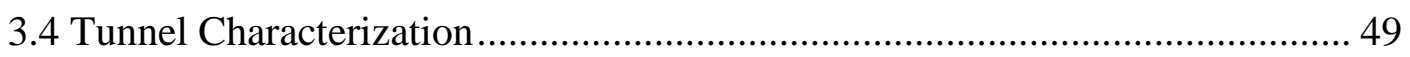

3.5 Conducted Experiments ............................................................................. 56

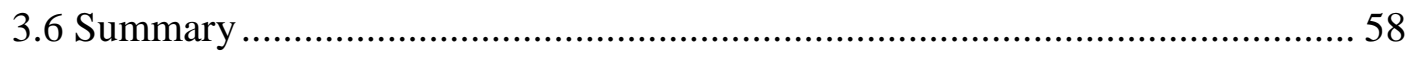

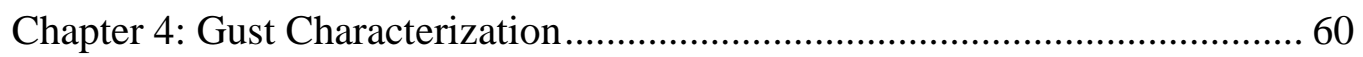

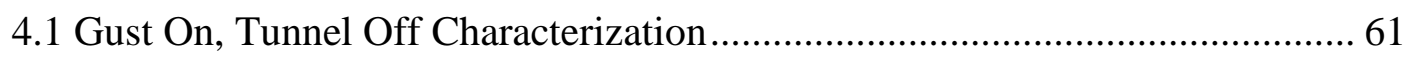

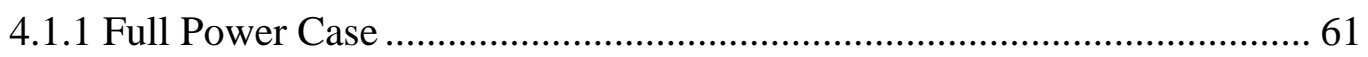

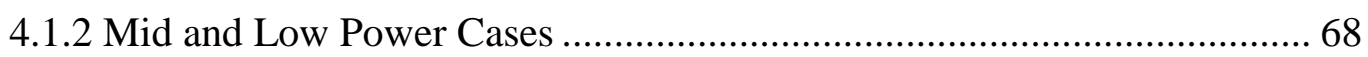




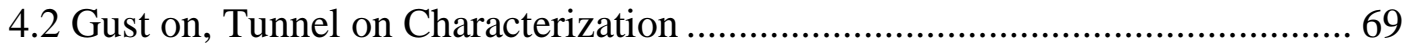

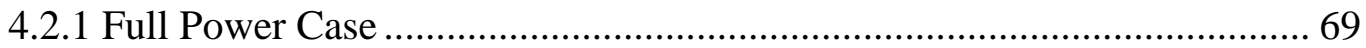

4.2.2 Mid and Low Power Cases ................................................................... 79

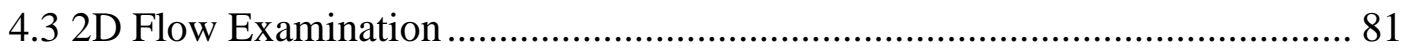

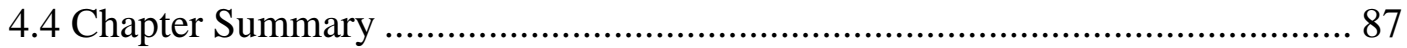

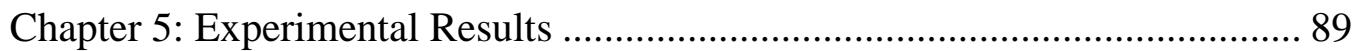

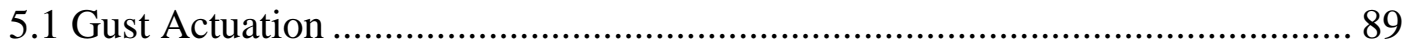

5.1.1 Full Power Phased Gust Actuation ........................................................ 90

5.1.2 Mid and Low Power Phased Gust Actuation............................................. 96

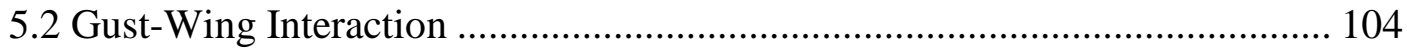

5.2.1 Full Power Gust-Wing Interaction, 0 Degrees......................................... 106

5.2.2 Full Power, 0 Degree Effective AoA Case Compared to Static Wing .... 109

5.2.3 Power 5 and 10-degree Effective AoA cases with Static Wing Comparison

5.2.4 Mid Power Gust-Wing Interaction......................................................... 121

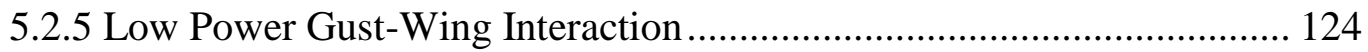

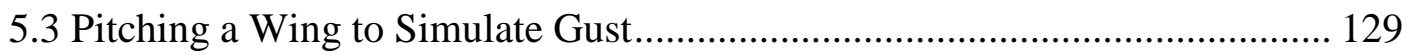

5.3.1 0-degree Initial Angle Pitch Maneuver.................................................. 129

5.3.2 5 and 10-degree Initial Angle Pitch Maneuvers ...................................... 140

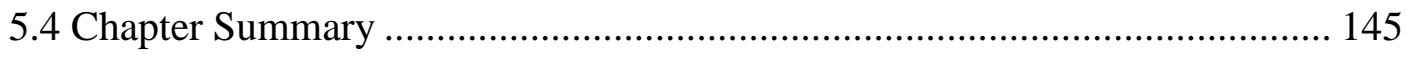

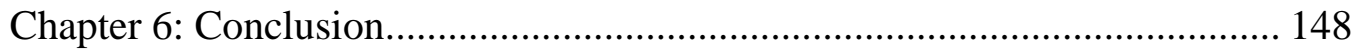

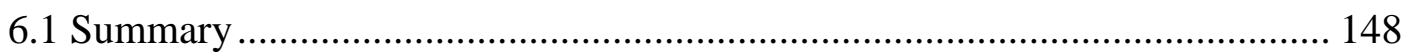

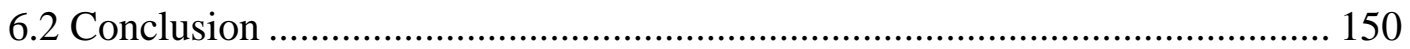

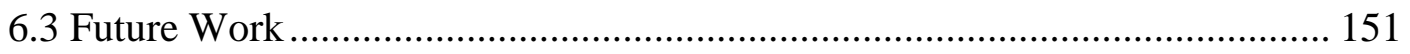

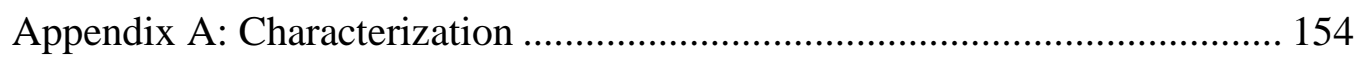

A.1 Gust on Tunnel Off Mid and Low Power Cases ........................................ 154

A.2 Gust on Tunnel on Mid and Low Power Cases ......................................... 163

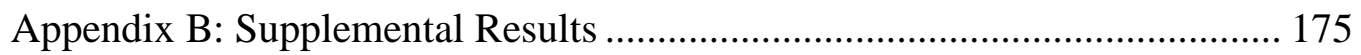

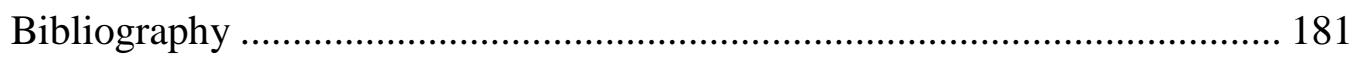




\section{List of Tables}

Table 1: UAS groups defined by the Department of Defense [12]........................... 2

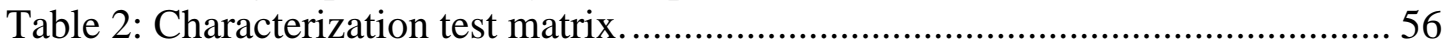

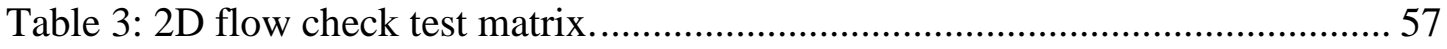

Table 4: Gust wing interaction and actuated gust cases. ...................................... 58

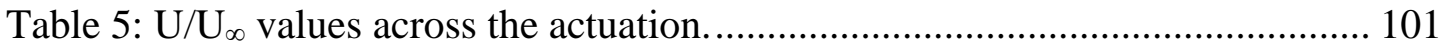

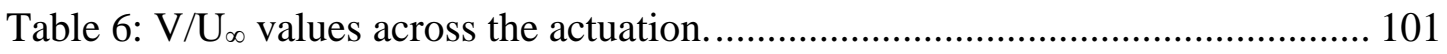

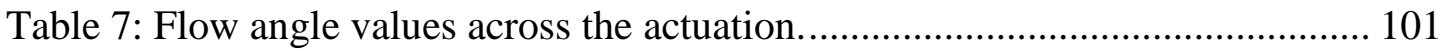

Table 8: Selected $t^{*}$ values from actuation process.............................................. 103 


\section{List of Figures}

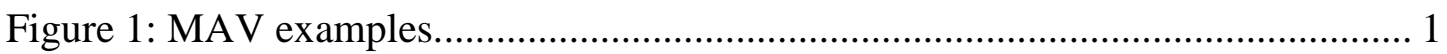

Figure 2: Examples of fans used for MAV testing indoors........................................ 8

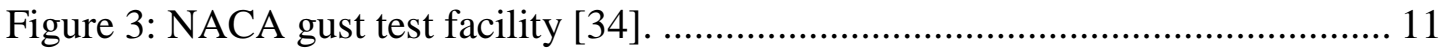

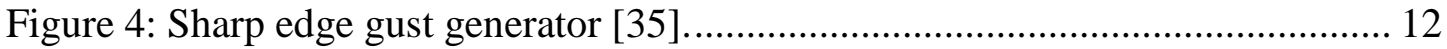

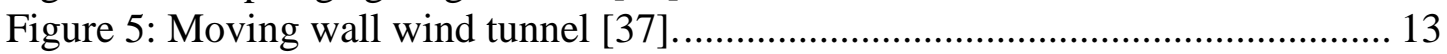

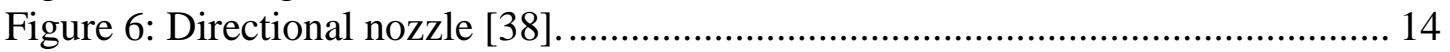

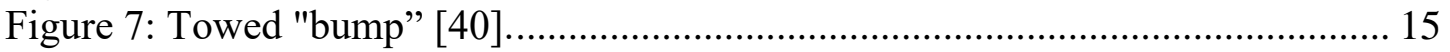

Figure 8: Active grid turbulence apparatus [44] .................................................... 17

Figure 9: Sketch of oscillating vanes [39] .......................................................... 18

Figure 10: Oscillating vane tunnel insert [39]..................................................... 18

Figure 11: Semi-span vanes [45] .................................................................. 20

Figure 12: Circulation controlled airfoil [49] ...................................................... 22

Figure 13: Sketch of Perrotta's flow from gust generator [73] ................................ 27

Figure 14: PIV results of a wing entering a gust from Perrotta and Jones [73]......... 28

Figure 15: Top-hat gust generator design [74] ....................................................... 29

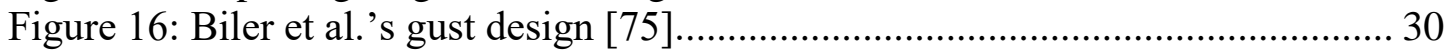

Figure 17: Vertical flow near top of building. ........................................................... 36

Figure 18: 3D model of the MAWT Facility......................................................... 37

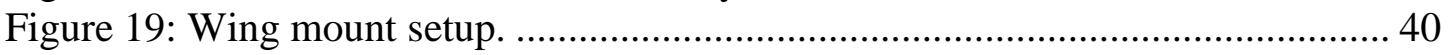

Figure 20: Initial sketch of gust generator............................................................ 41

Figure 21: Top view example fan nozzle position and size. Image to scale................ 42

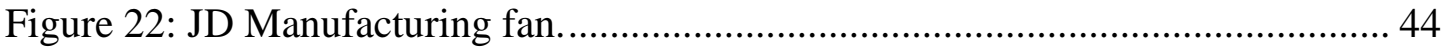

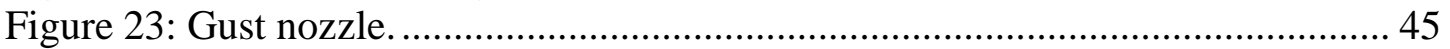

Figure 24: Gust entrance location on tunnel floor. .................................................... 46

Figure 25: 3D model of final setup with wind tunnel missing. .................................. 47

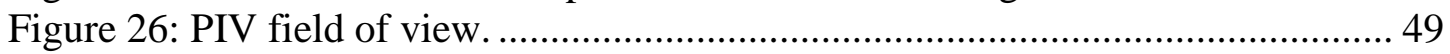

Figure 27: $\mathrm{U} / \mathrm{U}_{\infty}$ contour of the tunnel freestream..................................................... 50

Figure 28: $\mathrm{V} / \mathrm{U}_{\infty}$ contour of the tunnel freestream.................................................... 51

Figure 29: Tunnel freestream RMS initial characterization. ................................... 52

Figure 30: Tunnel freestream characterization with updated $\Delta \mathrm{t}$.................................. 54

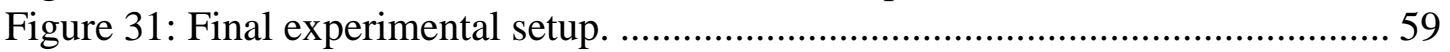

Figure 32: PIV laser locations on the wing, looking from above................................ 60

Figure 33: V velocity contours of the full power gust, no freestream. ......................... 61

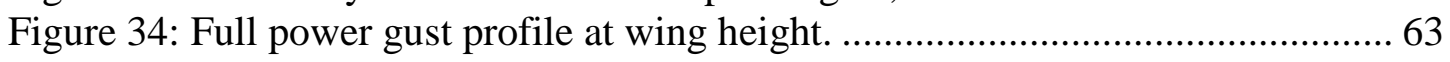

Figure 35: Vertical gust velocity vs. height in tunnel. Full power case....................... 64

Figure 36: U velocity contour of full power vertical gust, Position 1, no freestream. 66

Figure 37: Full power vertical gust RMS values. Position 1..................................... 67

Figure 38: V contour of various vertical gust powers. Position 1. ..............................6 68

Figure 39: Steady state gust freestream interaction, full power................................... 69

Figure 40: Extracted $U / U_{\infty}$ velocities at wing location for full power case................. 72

Figure 41: Gust ratio for full powered gust. .......................................................... 73 
Figure 42: Gust ratio extraction at wing height for the full power case ................... 75

Figure 43: Flow angle for the gull power gust freestream interaction...................... 76

Figure 44: Flow angle data extraction at wing height for full power case. ................ 77

Figure 45: RMS values for the full power gust at steady state at position 1. ............. 78

Figure 46: Full, mid, and low power comparisons for gust at Position 1................. 80

Figure 47: Change in U Velocity based on span location for full gust power............ 82

Figure 48: Change in V velocity based on span location for full power gust............. 83

Figure 49: Change in U velocity based on span location for mid power gust............ 85

Figure 50: Change in V Velocity based on span location for mid power setting. ...... 85

Figure 51: Change in U Velocity based on span location for low power gust. ........... 86

Figure 52: Change in $\mathrm{V}$ velocity based on span location for low power gust. ............ 86

Figure 53: Full-power actuated gust displaying contours of $\mathrm{U} / \mathrm{U}_{\infty}$ velocity.............. 90

Figure 54: Full power actuated gust displaying contours of gust ratio...................... 92

Figure 55: Full power actuated gust contoured to flow angle. ............................... 93

Figure 56: Flow properties vs. convective time for the full power gust case............ 94

Figure 57: Flow properties vs. convective time for the mid power gust case. ...........97

Figure 58: Flow properties vs. convective time for the low power gust case.............99

Figure 59: Vorticity contour for the gust at full power in an empty tunnel............. 104

Figure 60: Effective angle of attack in a gust. .................................................... 106

Figure 61: Actuating full power gust; wing at 0-degree initial effective angle of

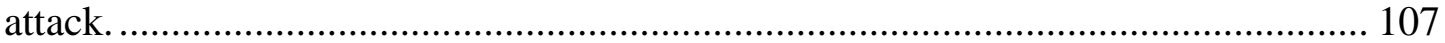

Figure 62: Actuating full power gust; wing at 0-degree initial effective, new

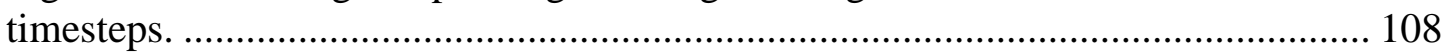

Figure 63: Static wing at positive angles of attack in lab frame of reference........... 110

Figure 64: Static wing at positive angles of attack in wing frame of reference........ 110

Figure 65: Pre full-power-gust/static wing comparison; 0-degree effective angle... 111

Figure 66: Early full-power-gust/static wing comparison; 5-degree effective angle.

Figure 67: Mid full-power-gust/static wing comparison; 13 and 15-degree effective

angles. .......................................................................................... 113

Figure 68: Late full-power-gust/static wing comparison; 13 and 15-degree effective

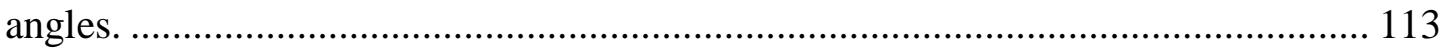

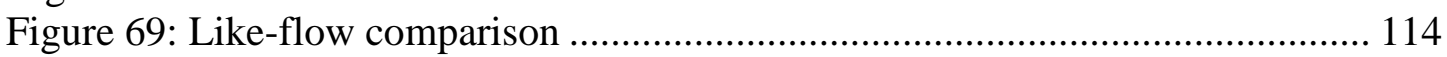

Figure 70: Actuating full power gust; wing at 5-degree initial effective angle........ 115

Figure 71: Pre full-power-gust/static wing comparison; 5-degree initial effective

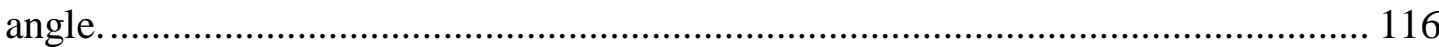

Figure 72: Early full-power-gust/static wing comparison; 10-degree effective angle.

Figure 73: Mid full-power-gust/static wing comparison; 5-degree initial effective angle.

Figure 74: Late full-power-gust/static wing comparison; 5-degree initial effective angle.

Figure 75: Actuating full power gust; wing at 10-degree initial effective angle...... 118

Figure 76: Pre full-power-gust/static wing comparison; 10-degree effective angle. 119 Figure 77: Early full-power-gust/static wing comparison; 15-degree effective angle. 
Figure 78: Mid full-power-gust/static wing comparison; 10-degree initial effective angle.

Figure 79: Late full-power-gust/static wing comparison; 0-degree initial effective angle.

Figure 80: Actuating mid power gust; wing at 0-degree initial effective angle. ...... 122

Figure 81: Pre mid-power-gust/static wing comparison; 0-degree initial effective angle.

Figure 82 Mid mid-power-gust/static wing comparison; 0-degree initial effective angle.

Figure 83: Late mid-power-gust/static wing comparison; 0-degree initial effective angle

Figure 84: Actuating low power gust; wing at 0-degree initial effective angle........ 125 Figure 85: Early low-power-gust/static wing comparison; 0-degree initial effective angle.

Figure 86: Late low-power-gust/static wing comparison; 0-degree initial effective

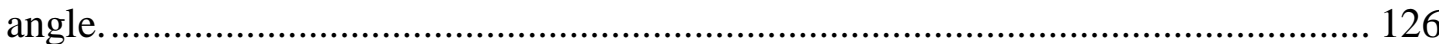

Figure 87: Actuating low power gust; wing at 5-degree initial effective angle........ 126 Figure 88: Early low-power-gust/static wing comparison; 5-degree initial effective angle.

Figure 89: Late full-power-gust/static wing comparison; 5-degree initial effective angle.

Figure 90: Actuating low power gust; wing at 10-degree initial effective angle...... 128 Figure 91: Early mid-power-gust/static wing comparison; 10-degree initial effective angle.

Figure 92: Late full-power-gust/static wing comparison; 10-degree initial effective angle.

Figure 93: Change in angle vs. convective time for the pitching wing. ................... 130

Figure 94: Wing pitched from 0 to 14 degrees to simulate full power gust. ............. 131

Figure 95: Comparison of pitching wing to wing in gust....................................... 132

Figure 96: Flow angle comparison for a wing pre-gust and in a steady gust. .......... 133

Figure 97: Gust ratio comparison for a wing pre-gust and in a steady gust. ............ 134

Figure 98: Estimated flow angle without gust's horizontal effect. ............................ 135

Figure 99: Comparison of pitching profiles......................................................... 136

Figure 100: Wing pitching from 0 to 10 degrees................................................... 137

Figure 101: Early gust-pitch comparison at 0-degree initial effective angle............ 138

Figure 102: Mid gust-pitch comparison at 0-degree initial effective angle............... 138

Figure 103: Late gust-pitch comparison at 0-degree initial effective angle. ............ 139

Figure 104: Wing pitching from 5 to 19 degrees to simulate full power gust. .......... 141

Figure 105: Mid gust-pitch comparison at 5-degree initial effective angle............... 142

Figure 106: Late gust-pitch comparison at 5-degree initial effective angle. ............. 142

Figure 107: Wing pitching from 10 to 24 degrees to simulate full powered gust. ... 143

Figure 108: Mid gust-pitch comparison at 10-degree initial effective angle............ 144

Figure 109: Late gust-pitch comparison at 10-degree initial effective angle. .......... 144

Figure 110: $\mathrm{U}$ velocity contour of full powered gust, no freestream........................ 154

Figure 111: V velocity contours of mid power gust, no freestream. ……………..... 155

Figure 112: Mid power gust profile at wing height. ............................................... 156 
Figure 113: Mid power gust strength vs height.

Figure 114: U velocity contour of mid powered gust, no freestream. .................... 158

Figure 115: Mid Power U and V RMS as percentage of nominal freestream at

Position 1.

Figure 116: V velocity contours of low power gust, no freestream........................ 159

Figure 117: Low power gust profile at wing height. .......................................... 160

Figure 118: U velocity contour of low powered gust, no freestream. ..................... 161

Figure 119: Low power U and V RMS as a percentage of nominal freestream flow at

Position 1.

Figure 120: Mid power gust with freestream displaying contours of $U / U_{\infty} \ldots \ldots \ldots \ldots . . . . .163$

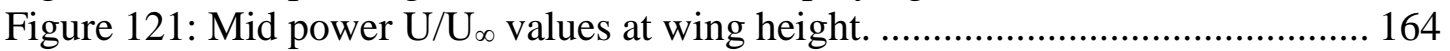

Figure 122: Mid power gust ratio contour with freestream................................ 165

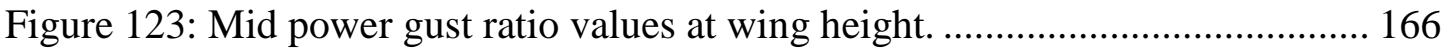

Figure 124: Mid power flow angle contours with freestream................................. 167

Figure 125: Mid power flow angle values at wing height. ................................... 168

Figure 126: Mid power U and V RMS as a percentage of nominal freestream flow at

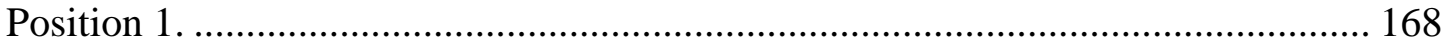

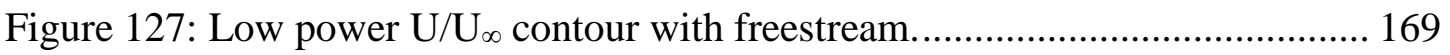

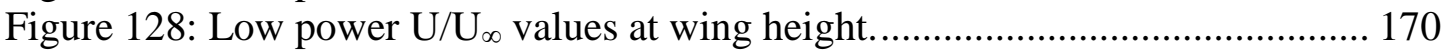

Figure 129: Low power gust ratio contour with freestream. ............................... 171

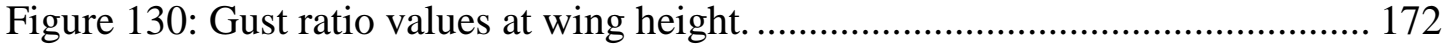

Figure 131: Low power flow angle contour with freestream. ............................... 173

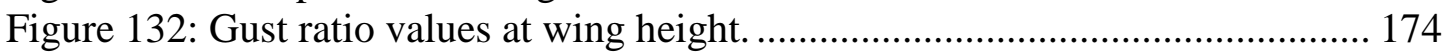

Figure 133: Low power U and V RMS as percentage of nominal freestream at

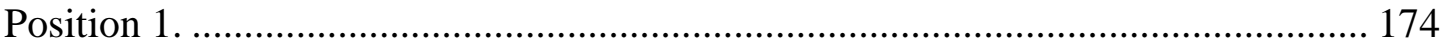

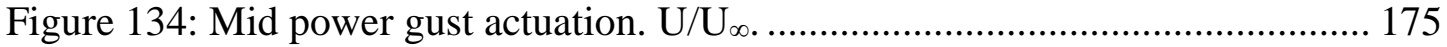

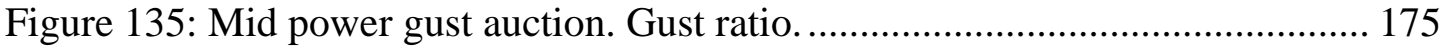

Figure 136: Mid power gust actuation. Flow angle. ............................................. 176

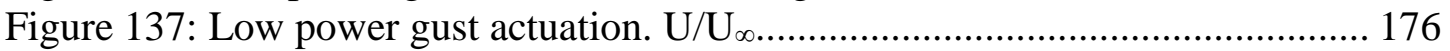

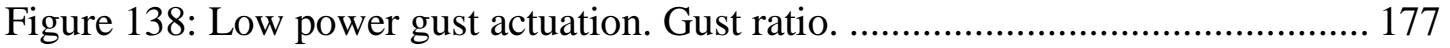

Figure 139: Low power gust actuation. Flow angle. ........................................... 177

Figure 140: High wing angles for a static wing, both lab and wing reference frames

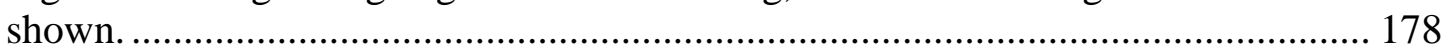

Figure 141: Actuating mid power gust; wing at 5-degree initial effective angle. .... 179

Figure 142: Actuating mid power gust; wing at 10-degree initial effective angle. .. 180 


\section{List of Abbreviations}

\begin{tabular}{ll}
$\alpha$ & Effective Angle of Attack \\
$c$ & Wing Chord, Meters \\
$c f m$ & Cubic Feet Per Minute \\
$t$ & Time, Seconds \\
$t^{*}$ & Convective Times, $\frac{U_{\infty} c}{t}$ \\
$\omega$ & Pitching Frequency \\
$x$ & Freestream Flow Direction \\
$y$ & Normal to Freestream Flow Direction \\
$A o A$ & Angle of Attack \\
$A R L$ & Army Research Laboratory \\
$C F D$ & Computational Fluid Dynamics \\
$D A R P A$ & Defense Advanced Research Projects Agency \\
$F D M$ & Fused Deposition Modeling \\
$F V M$ & Field Velocity Method \\
$H W A$ & Hot Wire Anemometer \\
$L E S$ & Large Eddy Simulation \\
$L E V$ & Leading Edge Vortex \\
$M \infty$ & Mach Number of Freestream \\
$M A V$ & Micro Air Vehicle \\
$M A W T$ & Microsystem Aeromechanics Wind Tunnel \\
$M D F$ & Medium Density Fiberboard \\
$N A C A$ & National Advisory Committee for Aeronautics \\
$N A S A$ & National Aeronautics and Space Administration \\
$P I V$ & Particle Image Velocimetry \\
$R M S$ & Root Mean Squared \\
$T E V$ & Trailing Edge Vortex \\
$U$ & Velocity of the flow in the horizontal direction \\
$U \infty$ & Freestream Velocity, m/s \\
$U A S$ & Unmanned Air System \\
$U A V$ & Unmanned Aerial Vehicle \\
$V$ & \\
\hline$U_{\infty}$ & Gelocity of the flow in the vertical direction \\
&
\end{tabular}




\section{Chapter 1: Introduction}

\subsection{Motivation}

There is an ever-increasing push to make aerial drones smaller and more compact. In 1995, the Defense Advanced Research Projects Agency (DARPA) created several grant programs and industry design incentives to spur the creation of a new class of air vehicles, which they termed Micro Air Vehicles (MAVs). Some of the results of this program can be seen in Figure 1. MAVs have become increasingly popular for a variety of activities from surveillance to defense to agricultural endeavors, but they are still most commonly used for recreational purposes [1].

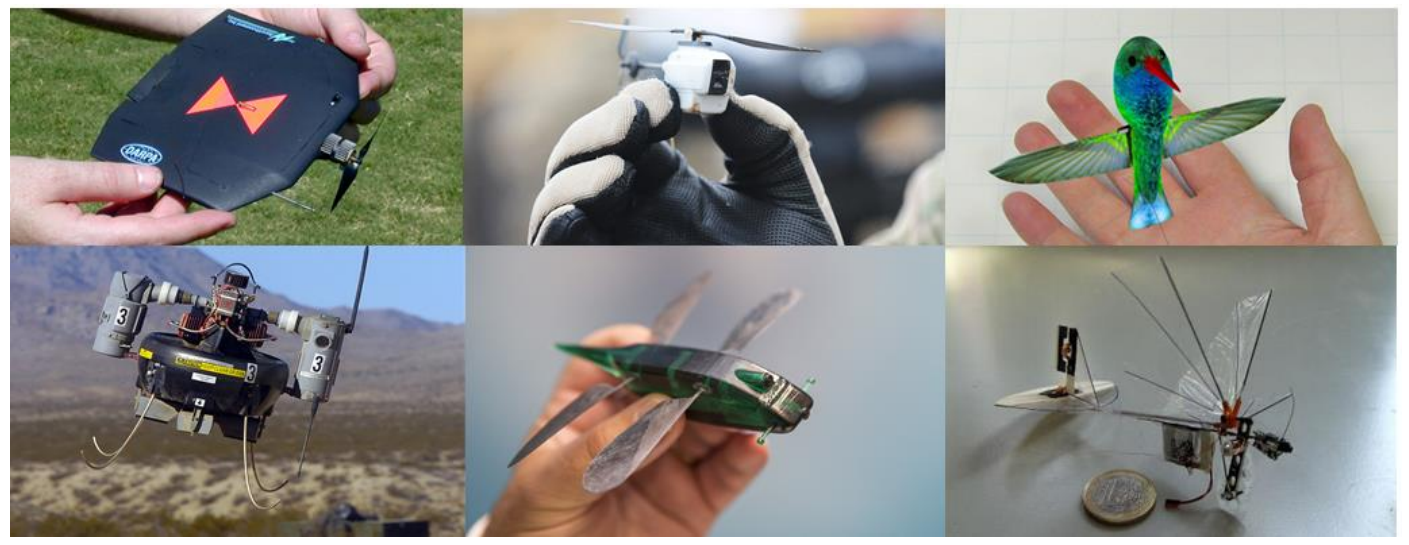

Figure 1: MAV examples.

Top left: Black Widow [2], Top center: PD-100 Black Hornet [3], Top right: Nano Hummingbird [4], Bottom left: T-Hawk [5], Bottom center: Animal Dynamics' Dragonfly [6], Bottom right: The Delfly Micro [7].

For a vehicle to be considered an MAV as outlined by DARPA, it was required to have a maximum dimension of $15 \mathrm{~cm}$ and weigh less than 100 grams including any payloads [8]. This would categorize all MAVs under Group 1 of the newer military Unmanned Air System (UAS) grouping [9], given in Table 1. The maximum airspeed of the Group 1 UAS is given as 100 knots or $51.44 \mathrm{~m} / \mathrm{s}$. This would equate to a 
Reynolds number of $\sim 850,000$ (assuming an arbitrary chord length of $0.25 \mathrm{~m}$ and flight at standard sea level) for the largest aircraft in this group. Mueller notes that current MAVs have Reynolds numbers between 10,000 and 100,000 [1], putting them on the lower side of Group 1. It is likely that future UAV groupings defined by the military will include a group for just MAVs due to their increased prevalence in the military.

Table 1: UAS groups defined by the Department of Defense [12].

\begin{tabular}{|l|c|l|c|}
\hline $\begin{array}{c}\text { UAS } \\
\text { Category }\end{array}$ & $\begin{array}{c}\text { Max Gross } \\
\text { Takeoff Weight }\end{array}$ & Normal Operating Altitude (Ft) & \multicolumn{1}{|c|}{ Airspeed } \\
\hline Group 1 & $<20$ pounds & $<1200$ above ground level (AGL) & $<100$ Knots \\
\hline Group 2 & $21-55$ pounds & $<3500$ AGL & $<250$ Knots \\
\cline { 1 - 1 } Group 3 & $<1320$ pounds & $<18,000$ mean sea level (MSL) & \\
\cline { 1 - 1 } Group 4 & $>1320$ pounds & & \\
\cline { 1 - 1 } \cline { 4 - 5 } Group 5 & & $>18,000 \mathrm{MSL}$ & \\
\hline
\end{tabular}

As UAS designs became smaller, their forward flight velocity began to decrease as well [1]; and thus, these new aircraft became more susceptible to unsteady environments [10]. Unlike passenger aircraft that see typical updrafts of less than 5\% of their flight speed [11] [12], MAVs encounter gusts of the order of their flight speed. One of the possible outcomes desired for a gust interaction is that in the presence of a gust, these vehicles will be able to adjust their flight path or wings to negate the effect of the gust. However, this is currently not the case in commercially available MAVs. A relatively small updraft $(<1 \mathrm{~m} / \mathrm{s})$ can cause an MAV to rapidly gain altitude, flip, and ultimately crash. A similar updraft would have minimal impact on the flight of a full-scale aircraft; and thus, the control response for that case can be approximated by a linear disturbance model [13] [14]. Recent work has shown that MAVs could also use large gusts similar to how a glider uses a thermal and climb in altitude purposefully while maintaining stability [15]. This would help to increase the energy efficiency of an MAV and could significantly increase total flight time. 
Theoretical control models for MAVs are common but often ignore the aerodynamics governing the physical gusts impinging on the MAV. This is in part due to a lack of understanding of the aerodynamics at these scales. By ignoring some of the physics involved, the control models lack both aerodynamic and propulsive efficiency needed for extending MAV flight times. Fundamental studies on gust encounter aerodynamics are key toward the development of robust control response models for future MAVs.

MAVs are often flown indoors for demonstration purposes. However, once these aircraft are taken out of their indoor test conditions and introduced to the natural environment, they quickly lose performance and efficiency. This is caused by the complexities of the natural flying environment at small scales. Flying animals constantly change their wing geometry and flight motions to accommodate for changes in flow conditions [16] [17] [18]. To truly gain maximum flying efficiency at these scales, MAVs must also become dynamic systems with active feedback and control. Before engineers can determine how to make an MAV change to accommodate unsteady flows, it must first be determined how these unsteady flows interact with key MAV components, like wings or rotors. The research being presented here looks at one such unsteady flow an MAV may encounter: a vertical gust scenario. This study evaluates the creation of a novel vertical gust generator in a wind tunnel and its subsequent interaction with a wing. Further exploration is done by comparing the flow field created by this gust generator to that of a pitching wing.

While aircraft response to a gust has long been the subject of discussion and research, many past studies have focused on full scale aircraft and neglected the 
extreme unsteadiness to which MAVs are subject. Küssner notes that the first gust encounter studies were conducted as early as 1914 [19]. Even with the long history of research, there is a significant lack of literature when it comes to fundamental fluid dynamic experimentation on MAV scales in relation to gust resilience. The flow structures around the wing and the effect of a gust on unsteady aerodynamic forces and moments has not yet been realized. This is at least in part because it is difficult to design a test apparatus that properly simulates an MAV-gust interaction. A lack of MAV scale experiments has led to much speculation as to what happens when the updraft magnitude is comparable to that of the vehicle's forward flight velocity. The current research study aims to tackle these issues by generating a controlled vertical gust in a wind tunnel specifically tailored to MAV scales.

\subsection{Thesis Outline}

The current report is separated into six chapters, of which this introduction to the problem at hand is the first. The second chapter is a literature review highlighting previous attempts to study gust interactions at both full scale and MAV scales. The third chapter is a description of the experimental method used to study the gust for this project. The fourth chapter presents and discusses the data collected from the characterization of the gust generator. The fifth chapter contains the results from the actuation of the gust both with and without a wing in the flow. These results are then compared to both a static and pitching wing in a gust-free environment. The sixth and final chapter is a conclusion of the results gathered from this project, a discussion of the overall success of the gust generator, and a short description of some future works planned or suggested for this apparatus. 
The present research provides several key advancements to the study of MAVscale gust interactions and gives some insight into how to compare a moving-fluid problem to that of a moving body. Key contributions of the current work include:

1. The creation of a novel gust generator in a wind tunnel resulting in a vertical gust with a maximum speed equal to $40 \%$ of the nominal freestream.

2. The breakdown of flow field evolution over time of a gust in a low Reynolds number flow around a wing.

3. The comparison of the gust-wing interaction to that of a pitching wing. 


\section{Chapter 2: Background}

\subsection{Previous Gust Studies}

Gust testing has been performed for over a hundred years, so numerous test apparatuses have been developed. This section will provide an outline of numerous test setups and devices followed by a review of the devices' application to the specific problem being analyzed. For another extensive overview, the author recommends the paper written by Murrow et al. [20] which contains a chronological review of previous gust facilities and gust studies from 1915 to (their) present in 1989. The following review covers the key works through this time period and continues through more modern efforts.

\subsubsection{Full Scale and Outdoor Testing}

Designers of MAVs often require evidence that their aircraft will be suitable for at least a minimally gusty environment. An obvious solution to this is to simply fly the aircraft outside in somewhat windy conditions and attempt to record flight test data where possible. This testing method is difficult as one cannot easily conduct real-time field tests of MAVs for gust response because data transmitters are too heavy for MAV scales and unexpectedly large disturbances could cause the vehicle to crash, rendering the test useless. For larger designs lacking the MAV classification and significant available payload weight, it is possible to install a telemetry device on the aircraft to extract flight data. However, to extract a full envelope of flight dynamics and performance information, a full battery of sensors is required. In one such case, Jordan

et al. used a telemetry system to collect flight dynamics characteristics for an aircraft 
with a seven-foot wing span; a span far outside the MAV range [21]. With a slightly smaller data recording system, Usui et al. were able to test the flight characteristics of a $1.82 \mathrm{~m}$ wing span aircraft [22]. Just the transmitter for these types of systems was around 15 grams, or $\sim 15 \%$ of many MAV's total flight weights. Depending on which sensors are required for testing, the sensor alone could easily exceed the 100-gram flight weight required for the "MAV" designation [23]. Therefore, it is clearly not possible to fly an MAV with the current recording devices needed to collect the full dynamic characteristics of the aircraft. Even if these options were to become smaller in the future, their weight would significantly alter the flight dynamics of the vehicles being tested. Furthermore, these outdoor tests often require FAA approval, substantial amounts of unrestricted airspace, or even outdoor netted facilities for safety; all of which are hard to obtain or expensive to build.

The ability to capture flow data will also still be missing from such tests. Adapting current flow field measurement techniques to outdoor settings and flight tests is an extremely complicated feat. There are many variables that cannot be controlled in such a setting, and with techniques such as particle image velocimetry, safety becomes a large issue. Capturing the flow field is crucial to the advancement of the aerodynamic development of such craft and therefore should not be over looked when selecting a test procedure or apparatus for flight ready vehicles.

Another testing strategy for MAVs has been to fly them indoors, but over large fans to simulate gusts. Examples of fans used in this way can be seen in Figure 2. Flying indoors past or over artificial gusts (i.e. fans) is more controlled than outdoor flying and does provide a somewhat controllable gust interaction. It is also easier to set up a 
motion capture system in indoor spaces, outlined by How et al. in their work describing their test facility [24]. These motion capture systems have allowed researchers to capture the flight of the aircraft and use computer programs to isolate some vehicle dynamics from the flight path and wing motion of the vehicle. However, these types of tests do not typically allow for the collection of force measurements or flow data; both of which are critical in order to create better MAV designs. They only show how an already designed and built aircraft responds to unsteady gust-like environment. There is also doubt as to the repeatability of such a test as commercially available fans are not manufactured to high tolerances and room boundary conditions make any quantitative comparisons difficult.

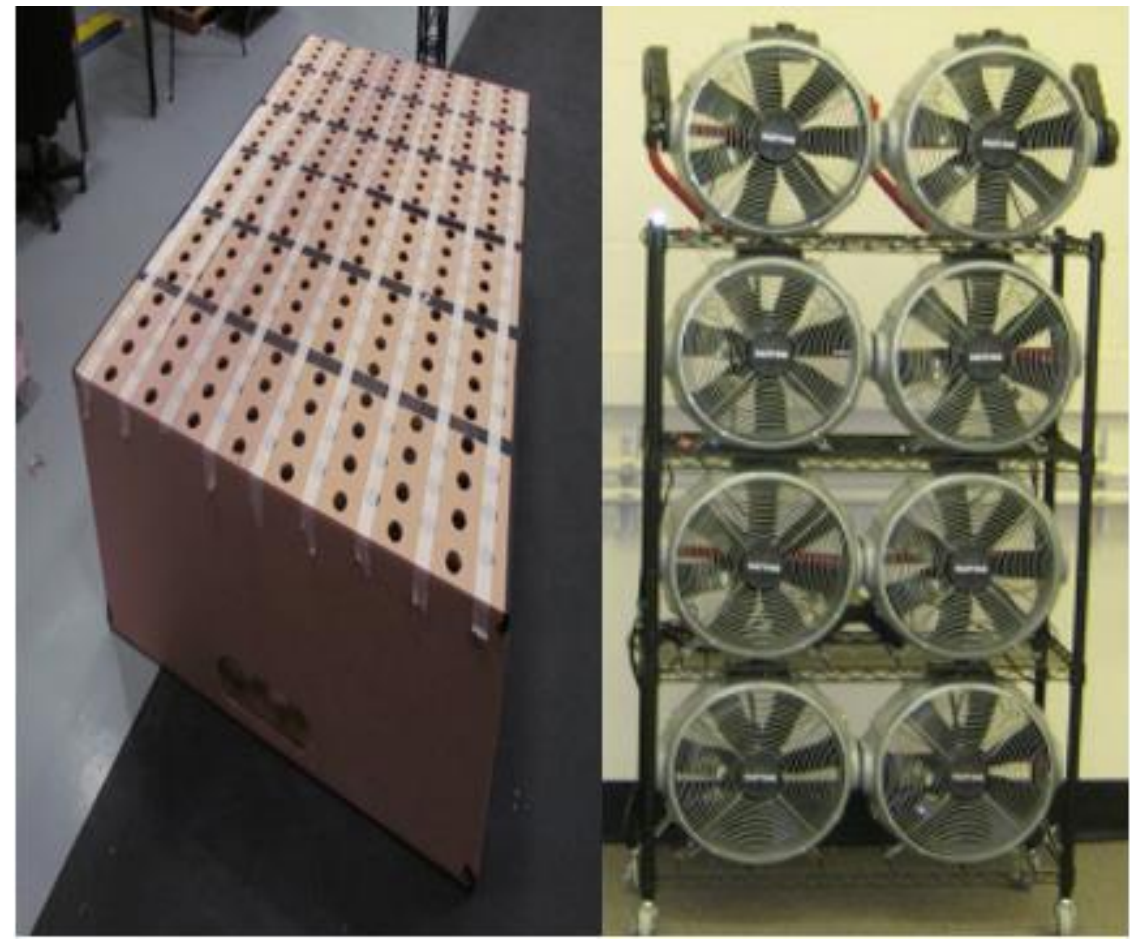

Figure 2: Examples of fans used for MAV testing indoors. Left: stand mounted horizontal gust [25], Right: floor mounted vertical gust [15].

NASA has also developed a test apparatus for larger UAS designs that allows the user to attach the article or aircraft on which testing is desired to an F-104 [26]. This 
allows the aircraft to experience speeds and altitudes it may experience in flight without flying under its own power. This method helps to confirm the objects' structural design and measure some forces the vehicle will experience. Unfortunately, these tests need to take place at flight speeds and altitudes far out of the range for which MAVs are designed, and again lack any flow field evaluation. There may be potential to conduct similar tests with an unpowered MAV tethered in a similar way to a larger drone that then flies at MAV-like conditions. However, this review did not find evidence of any such tests being conducted.

The final test apparatus that is becoming increasingly popular is simulation/ simulator environments [27]. Sivakumar and Tan [28] used X-Plane [29] to simulate a follower-leader UAV swarm in windy conditions. The simulation results provided their team with data that predicted the flight path of aircraft and captured some flight dynamics. However, this work lacked comparable field testing to validate their results. Murch et al. [30] were able to create a test plane with off-the-shelf parts and collected flight test data from this aircraft to validate an in-house flight simulation by comparing gathered flight dynamics information to that of the simulator. Work by Gavrilets et al. took a similar approach with an X-cell 60 helicopter, which was used to validate inhouse simulations for use in determining the flight characteristics [31]. However, as with other methods, simulation results will not capture flow field data, leaving a gap in fundamental vehicle response behavior. This review was unable to find a simulation that was compared to actual flight test data from highly unsteady or turbulent flight conditions. This leads to some doubt as to the reliability of simulation results once the models are introduced to increasingly unsteady environments. 


\subsubsection{Novel Gust Creation Methods}

As stated, experimental research into gust interactions with aircraft have taken place for over 100 years. Throughout this time, researches have created a large range of apparatuses with the hopes of best testing these gust interactions. Each researcher took a different approach based on their end goal or the flight conditions their vehicle of interest would experience. This section highlights some of the more novel techniques that have been used over the years and the various outcomes created.

Küssner's work, along with work done by Rhode and Lundquist, simply monitored gust loads of an aircraft in flight [19] [32]. Their work focused on large scale aircraft and because of this, they assumed gusts were simply "changes in lift caused by changes in angle of attack and relative airspeed." Furthermore, the work was largely interested in structural design for gust loads and not so much the aerodynamics of a gust encounter. Following the desire for more studies of the impacts of gusts on aircraft structures in a more controlled setting, NACA built the first experimental gust set up [33], shown in Figure 3. This design worked by rolling the test model down a ramp and more or less launching it into free flight across a large open fan and into a netted catch rig. The fan was controlled to produce gusts between 2 and $10 \mathrm{ft} / \mathrm{s}(0.61$ to $4.57 \mathrm{~m} / \mathrm{s})$. For these gust speeds, the model was typically launched at $60 \mathrm{ft} / \mathrm{s}(18.29 \mathrm{~m} / \mathrm{s})$ and the researchers specified Reynolds numbers between 125,000 and 180,000 for their tests. In one test case however, a Reynolds number of 85,000 was achieved by launching the model at $40 \mathrm{ft} / \mathrm{s}$. The models tested are diagramed in a figure in the report, but due to the image quality, the specific wing dimensions cannot be determined. Assuming standard sea level properties; however, it can be estimated that the models tested had 
chords between 4 and 6 inches based on the Reynolds numbers and flight velocities specified. This study was mostly interested in the force seen by the wing for structural considerations; and therefore, other properties were not measured. It was concluded that the force increase on the wing was linearly dependent on gust velocity. This is one of the best ways of experimenting with gusts to date. The vehicle flies under its own power into a gust, the flow field can be captured with modern techniques, and presumably the gust strength and shape can be altered with a little modification of the test rig. Obviously, such a system has its drawbacks, such as space requirements and the inability to test just a wing model, but the aircraft was able to experience all the forces it would while flying into a gust. This setup was used for various other tests including Reisert's work to verify a scale model of the XBDR-1 Airplane [34].

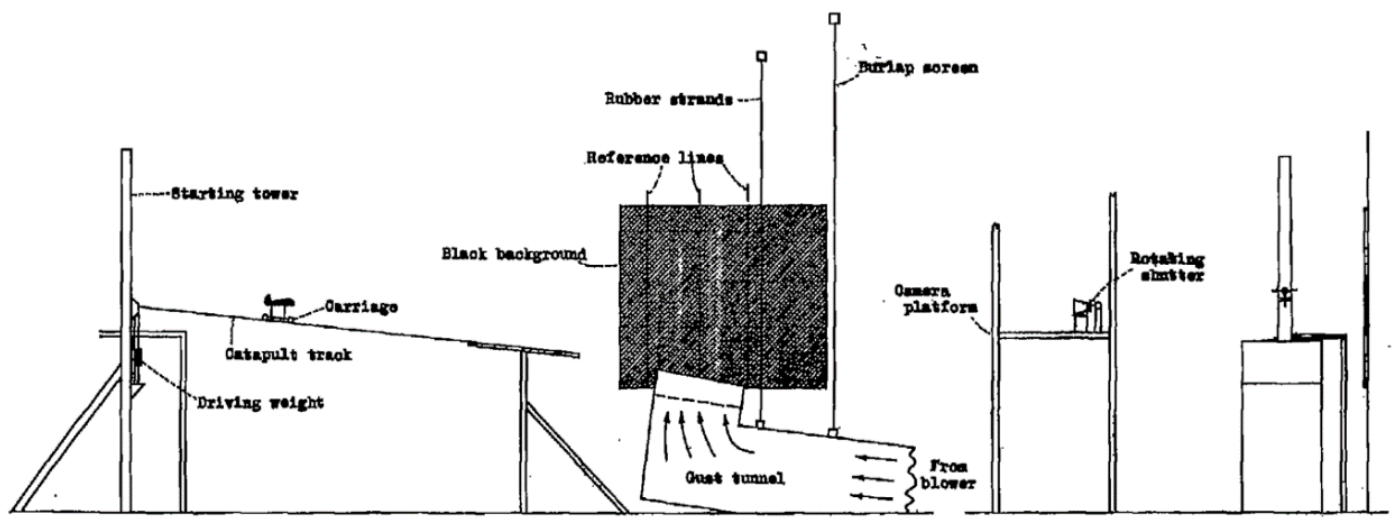

Figure 3: NACA gust test facility [34].

Not all past experiments focused on fixed wing aircraft. Maglieri and Reisert developed a system that would test a helicopter blade as it passed through a sharp edge gust [35]. A gust with a top-hat flow profile was desired for comparison with linear theories. This was achieved by sucking the air upwards as well as blowing from the bottom, as shown in Figure 4. However, the flow field for this gust was not evaluated and therefore the exact shape of the gust is not known. Gust velocities of 5, 7.5, and 10 
$\mathrm{ft} / \mathrm{s}(1.52,2.29,3.05 \mathrm{~m} / \mathrm{s})$ were tested. As with many of the previous tests, the primary focus and conclusions of this work was structural. Maglieri and Reisert determined that a teetering rotor saw less of the effects of the gust than a fixed-at-root rotor [35].

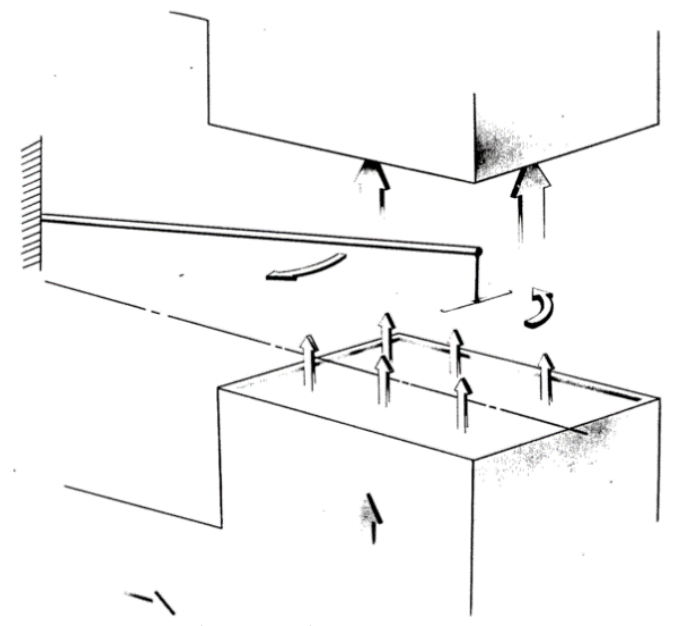

Figure 4: Sharp edge gust generator [35].

Hunt et al. published a report in 1961 for the Aeronautical Research Council in the UK that highlighted some unique gust experiments done on delta wing gust interaction [36]. Their set up involved attaching a delta-wing model to a sled and propelling it via rockets down a 2000 -foot-long track. At a point along the track, the delta wing passed in front of the open end of a wind tunnel blowing a jet of air across the path of the delta wing, simulating a gust. This produced a jet of air 15 feet wide and 4.5 feet deep. At the track centerline the mean velocity of this jet was $46 \mathrm{ft} / \mathrm{s}(14 \mathrm{~m} / \mathrm{s})$. Only pressure data was collected for this experiment. While this method does simulate the real-world gust encounter, such a large track and a strong gust is clearly not practical for testing at the MAV scale. Even if these parameters were to be scaled down, it is suspected that attempting to mount an MAV on any size track would hinder the ability to collect relevant data from such a test since the mounting hardware would largely affect the flow field of such a small craft. 
Holmes designed a wind tunnel from scratch with the purpose of trying to create both vertical and horizontal gusts [37]. In doing so he created a tunnel with actuated wall geometry, shown in Figure 5. The floor and roof of the tunnel could be moved up and down in phase to create a sinusoidal updraft in the tunnel, or out of phase to create a pulsing streamwise gust. The tunnel was characterized at both 27 and $54 \mathrm{ft} / \mathrm{s}(8.2$ and $16.5 \mathrm{~m} / \mathrm{s}$ ) and vertical gust ratios of up to $9.5 \%$ of the freestream were observed. While this setup was successful in producing the desired gusts, it requires a researcher to build a new tunnel from scratch in order to test gusts. Furthermore, it could not create a step or impulse gust profile at the test article, rather the article saw an oscillation of the freestream speed or direction.

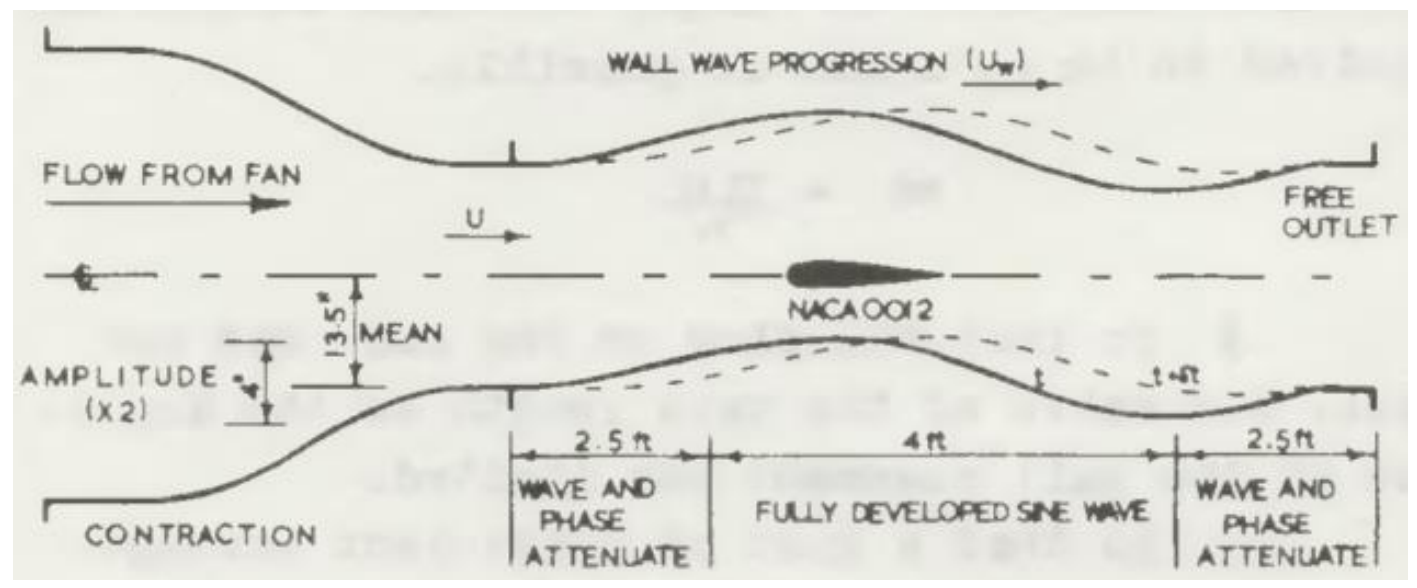

Figure 5: Moving wall wind tunnel [37].

Patel et al. put together a comprehensive paper outlining the development of a gust facility at Queen Mary College in London in 1977 [38]. The generator involved putting a directionally controllable nozzle at the contraction of an open return wind tunnel. This nozzle had flexible walls and was controlled by a hydraulic actuator that would deflect these walls and help to redirect the flow based on user inputs, as shown in Figure 6. For the initial characterization, the tunnel was run between 12 and $20 \mathrm{~m} / \mathrm{s}$ 
with a maximum gust amplitude of 35 degrees recorded with no test article in place. While this design did create impressive results, it most likely requires a new tunnel to be built by a researcher in order for it to be incorporated. Furthermore, the angle created by the nozzle is directly proportional to how close to the front of the test section the test article was. This means that the "gust" created was only an angle of attack change of the flow and not a vertical ( $\mathrm{V}$ component) velocity being introduced. Therefore, no increase in horizontal ( $\mathrm{U}$ component) velocity can be seen; and in fact, it is suspected that this component would have actually decreased as the flow was redirected.

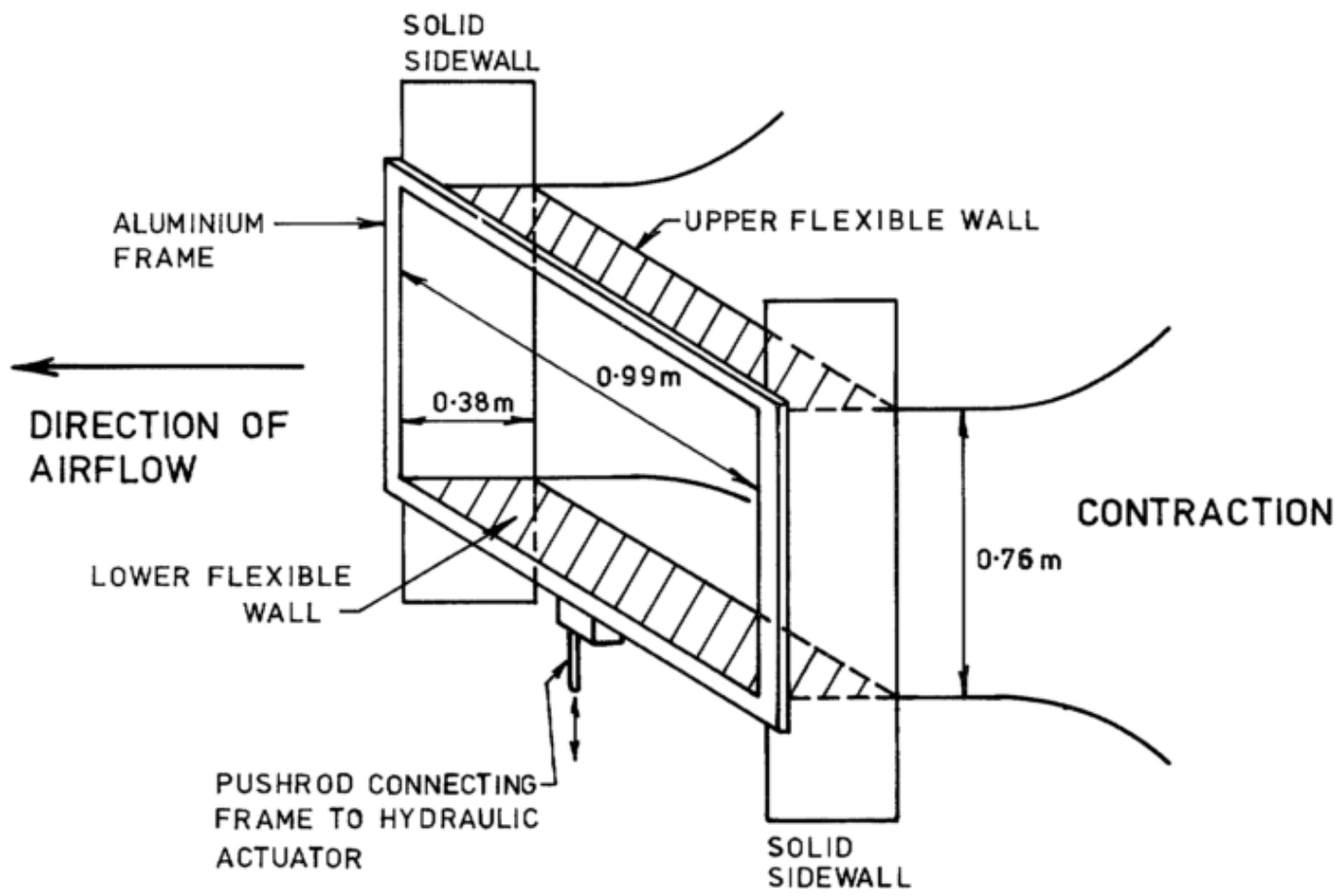

Figure 6: Directional nozzle [38].

Garby et al. and Kuethe et al. studied the use of a moving "bump" at the bottom of the tunnel, seen in Figure 7 [39] [40]. Their idea was to tow a full span rounded wedge on the bottom of the tunnel from an upstream position to a downstream one, passing under the test article. The tunnel was run at speeds of 20, 55, and $72.5 \mathrm{ft} / \mathrm{s}(6.1$, 16.8 , and $22.1 \mathrm{~m} / \mathrm{s}$ ), equating to a Reynolds number range of $40,000-148,000$ for the 
wing tested. The bump was towed at speeds of 10.1, 12.5, and $14.3 \mathrm{ft} / \mathrm{s}(3.1,3.8,4.4$ $\mathrm{m} / \mathrm{s}$ ). The goal of the bump towing system was to redirect the flow upwards as this bump passed under the test model. However, they discovered that this type of setup did not produce a strong vertical gust. In fact, it produced an increase in lift by acting as a blockage of the lower third of the tunnel, increasing the flow speed around the test article. It appeared that while the flow was directed around the wedge, some moving upwards, it was quickly redirected along the horizontal streamline and this "updraft" never reached the test article.

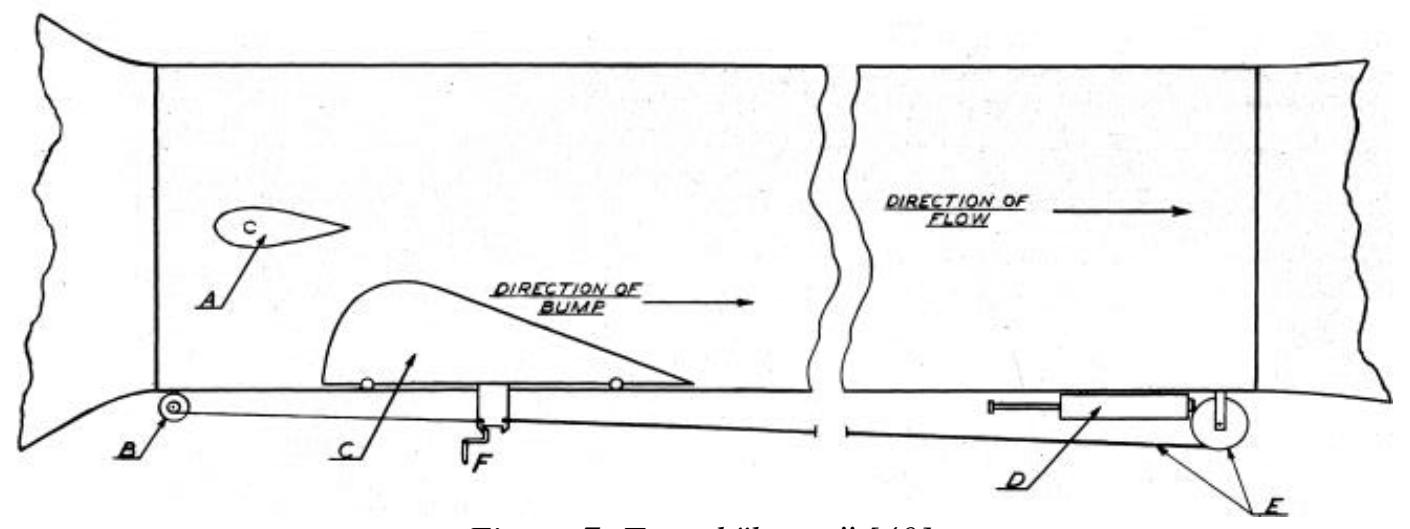

Figure 7: Towed "bump" [40].

Around the same time as Garby's work, Hakkinen et al. designed a gust system for NASA in a Massachusetts Institute of Technology wind tunnel [41]. This system used a large wing upstream of the test article that was "plunged" to create a shed vortex, which diffused while convecting downstream. This method created unsteady transverse velocities at their test wing location. Test cases were run at 50 and $79 \mathrm{mph}$ (22 to 36 $\mathrm{m} / \mathrm{s}$ ) and a NACA 0010 with a 1 -foot chord was chosen as the test subject. This resulted in Reynolds numbers between 500,000 and 1,000,000. Their results showed that an increase in lift was observed for the stationary wing as the plunged wing's reduced frequency was increased. However, a critical issue with such a setup was observed by 
Hakkinen: “...the magnitude of the airstream oscillation was small, and difficulty was encountered in distinguishing it from tunnel turbulence." It is expected that the oscillations were small due to the high tunnel speeds used for the tests. That being said, this method has been improved upon and is a method still being used for some MAV scale testing today [42] [43]. However, this design still cannot create exceptionally large flow angle changes or steady gusts.

In 2009, Roadman and Mohseni worked to tailor a wind tunnel specifically to testing MAVs [44]. To achieve this, they selected an active grid turbulence mechanism, Figure 8, which used actuated small slats to create the desired effect. These slats control when and how much flow was allowed into the tunnel at a time. It was thought that lateral gusts could be produced by choking the flow across the entire mouth of the tunnel while longitudinal gusts could be created by choking the flow on only the top or bottom half of the tunnel. For the characterization, the tunnel was run at $12 \mathrm{~m} / \mathrm{s}$. Hot Wire Anemometer (HWA) measurements showed high turbulence levels rather than gust-like behavior. The researchers highlighted the importance of creating an actual active gust generating system as opposed to having a turbulence generating one in future experiments since the system was limited in the strength of gusts it could create. 


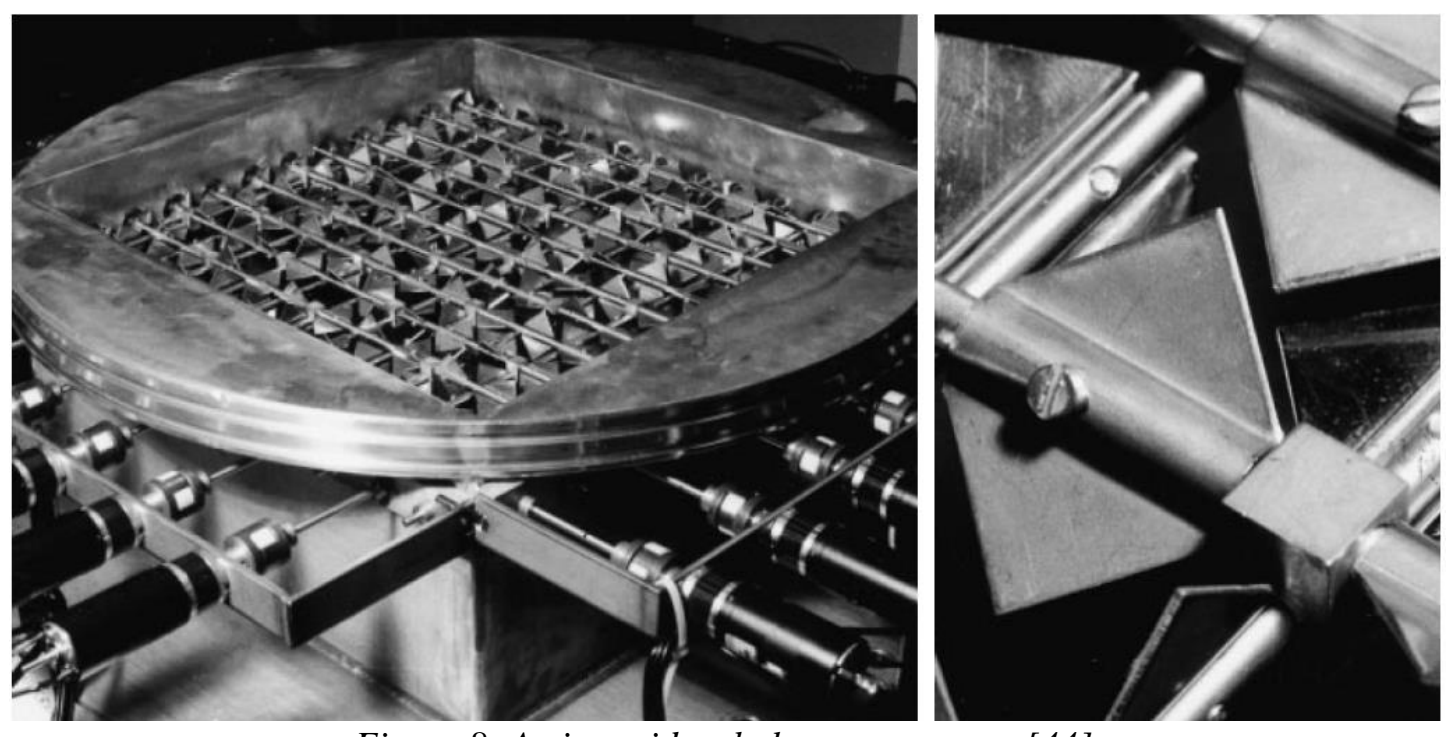

Figure 8: Active grid turbulence apparatus [44].

\subsubsection{Oscillating Vanes and Shutter Gust Generators}

The most commonly successful mechanisms used to create a gust in a wind tunnel are oscillating vanes (or airfoils) and shutter systems upstream of the test section. Such studies have been done as early as 1957 [39]. Garby et al. likely designed the first gust generator using oscillatory vanes, which they referred to as "Venetian Blinds", shown in Figure 9 and Figure 10 [39]. The vanes were attached to a motor and pitched from 0 degrees to 10 degrees and back in $1 / 25^{\text {th }}$ of a second, creating an oscillating up and downdraft at the test location. The vortex sheets shed by the slats during this motion passed downstream and created an artificial gust as they passed over the test article. It was shown that the slat actuation caused a 12-degree angle of attack change when the tunnel was run at $15 \mathrm{ft} / \mathrm{s}(4.6 \mathrm{~m} / \mathrm{s})$. Tests up to $60 \mathrm{ft} / \mathrm{s}(18.3 \mathrm{~m} / \mathrm{s})$, equating to a Reynolds number of $\sim 180,000$ for their model, were run and were noted to have created flow angle changes up to 10 degrees. One of the key issues that Garby et al. showed with their design is that there was no way of making an ever-present gust with this vortex 
generator, so quasi-steady values of the lift could not be obtained. Another issue with their design was that the wing experienced a flow angle change between -4 and -6 degrees just prior to the steep positive increase. This behavior is more akin to a largescale turbulent structure interaction than a true vertical impinging gust on an MAV.
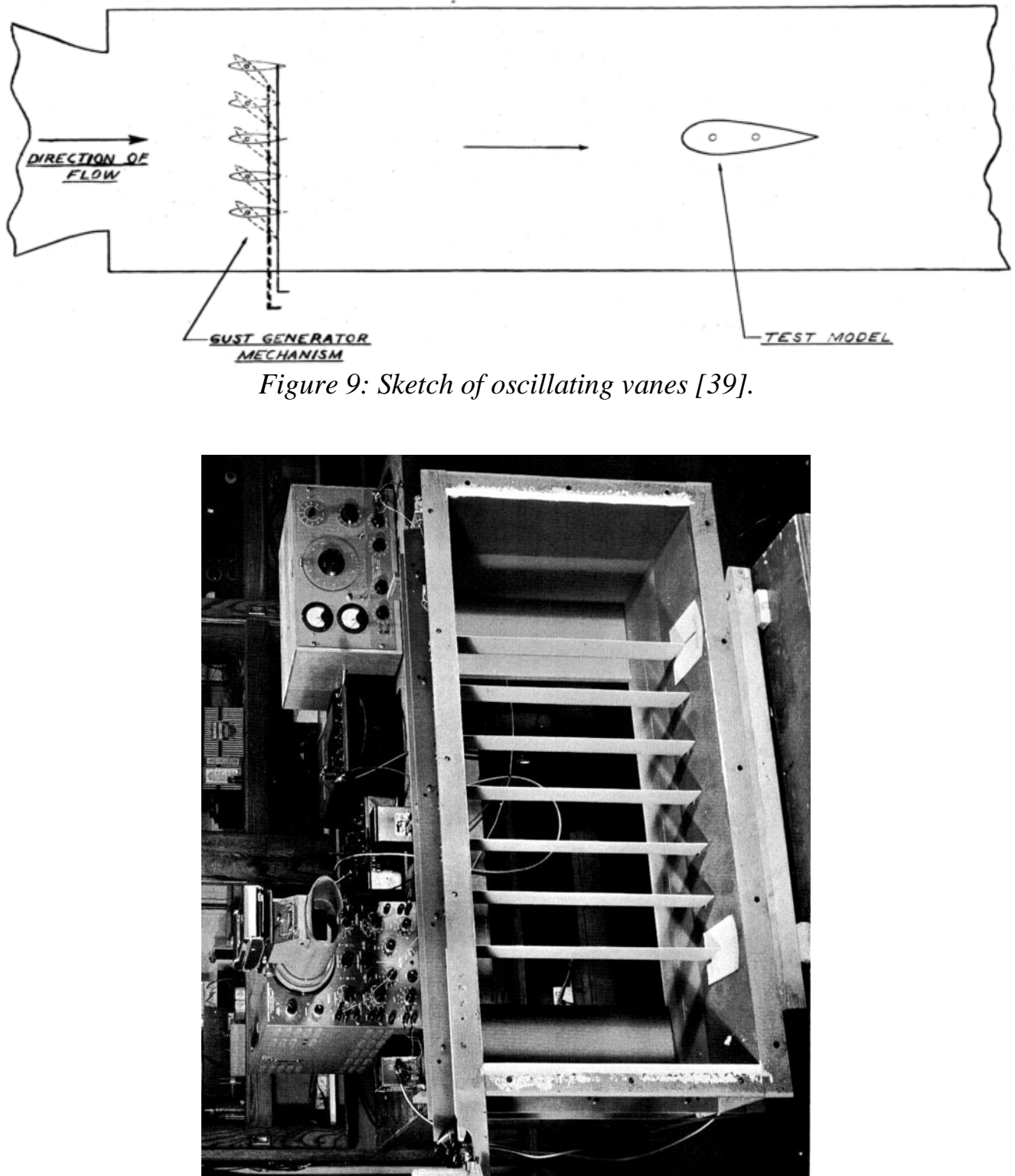

Figure 10: Oscillating vane tunnel insert [39].

A few years after Garby's work, Reid et al. attempted to build a similar configuration for NASA in the Langley low-turbulence pressure tunnel [45]. A pair of 
semi-span vanes were mounted in the throat of the tunnel and oscillated about the quarter chord between $\pm 13^{\circ}$ at rates between 2 and $20 \mathrm{~Hz}$, shown in Figure 11. Reid noted that because of the narrow rectangular cross section of this tunnel, the vanes were mounted on one wall only, and the opposite wall became a reflective plane. The vanes were 1 foot in span, which equated to one third of the tunnel width. For this configuration, the tunnel was run with Mach numbers between 0.39 and 0.80 , equating to a Reynolds number for their sting-mounted model between $18 \times 10^{6}$ and $38 \times 10^{6}$. It was shown that airstream angles of the order of $15 \%$ of the vane turning angle could be achieved as well as a constant amplitude and phase over about $20 \%$ of the tunnel width. This work was extended by Gilman et al. to study gust load effects in transonic conditions in the Langley transonic dynamic tunnel [46]. In this case, the semi-span vanes were mounted on both walls and the tunnel was run at Mach 1.0, equating to a Reynolds number of $\sim 45 \times 10^{6}$. A scale model was tethered inside the tunnel and changes in flow angles of about two degrees were achieved. In both cases, the tests produced what at the time were considered adequate flow angle changes at such high speed. But as mentioned earlier, at MAV scales, higher angle of attack changes are needed. 


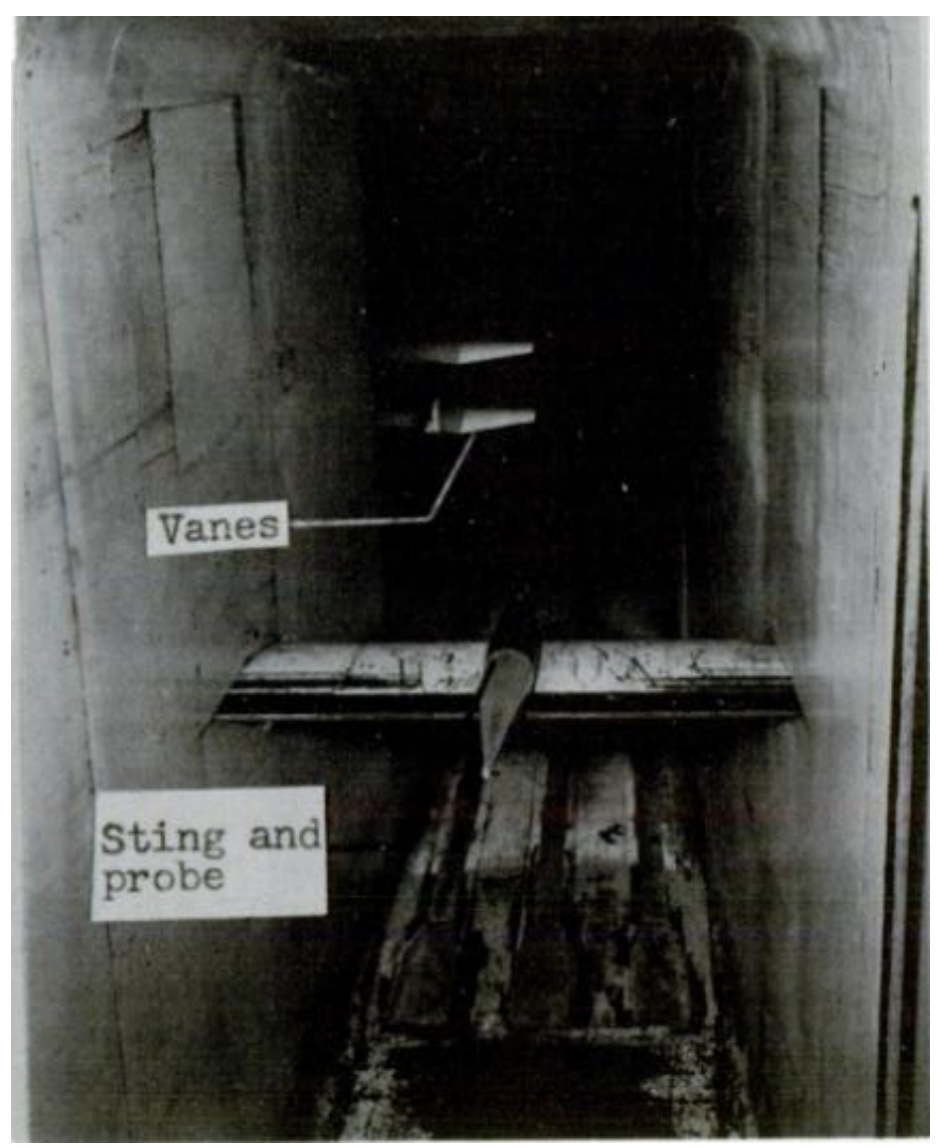

Figure 11: Semi-span vanes [45].

As understanding of the gust generating mechanisms improved, so did the end results of the generators being tested. In 1969, Buell et al. compared several vane configurations to determine an optimal case for their tunnel. They showed that by oscillating vanes with an amplitude of 16 degrees, they could obtain gust amplitudes of up to $45 \%$ of the free-stream velocity [47], but only under certain conditions. In this case, the tunnel was run between 50 and $250 \mathrm{ft} / \mathrm{s}$, or between 15.2 and $76.2 \mathrm{~m} / \mathrm{s}$, with no test article in place. However, due to the small portion of the test section that sees this vertical gust and the fact that the gust direction changes between positive and negative based on the sign of the vortex shed, Buell et al. concluded that oscillating 
vanes are not suitable for creating two or three-dimensional gusts representative of atmospheric turbulence.

In 1970, Umbarger [48] created gusts using the same principle but instead of using a mechanically controlled vane, his vanes were controlled with a jet being actuated over a trailing edge flap. The idea was that the actuated jet would be able to increase the lift generated by this airfoil flap, much like active flow control does today, and create a larger shedding vortex. The tunnel was characterized at speeds between 50 and $100 \mathrm{ft} / \mathrm{s}$, or 15.3 and $30.5 \mathrm{~m} / \mathrm{s}$. As with previous attempts, there was a fairly substantial change in angle of attack at the planned location of the test subject, about 11 degrees in this case, but the same issues presented themselves as in the study by Garby et al.

In 1974, Ham et al. developed a wind tunnel that used harmonic circulation control of twin parallel airfoils to achieve the harmonic lift variation required for gust generation [49]. Basically, this unique design pushed air out of an orifice at the trailing edge of a wing, where the trailing edge had been replaced by a cylinder; see Figure 12. The pitch angle of the airfoil was also controlled to create the desired gust effect at the test article. The tunnel was run at 40, 60, 82.5, 100, and 120 miles per hour $(17.9,26.8$, $36.9,44.7,53.6 \mathrm{~m} / \mathrm{s}$ ) and gust velocities of around $10 \%$ of the freestream were measured. The resulting gust was similar to that of more traditional oscillating vane experiments in that its motion was sinusoidal, but design complexity was a major drawback of this setup. 


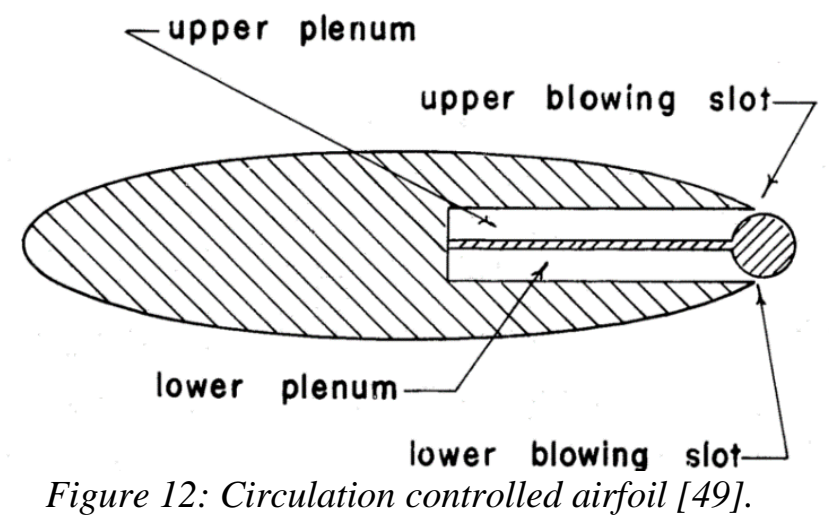

Tang et al. used rotating slotted cylinders placed in the trailing edge of airfoils to help create larger shedding vortices than standard oscillating vanes would [50]. A 12inch chord NACA 0012 was used as the test article and the tunnel was operated at 20 $\mathrm{m} / \mathrm{s}$. This equates to a Reynolds number of $\sim 400,000$. This experiment reproduced many of the same forces observed in oscillating vane experiments, as a sinusoidal updraft was seen to produce an increased lift of about $16 \%$, but overall the design was very complex in both creation and implementation.

Many more pitching vane designs have been created over time with the primary differences between each version being the setup specifications (pitching frequency, distance from model, freestream velocity, vane chord, number of vanes used, and tunnel size) and whether or not the entire vane pitches or just a trailing edge flap. While this type of experiment is a decent approximation of large scale aircraft-gust interaction, this method is not viable for MAV scale gust interactions since the gust to freestream ratios seen by MAVs are often much larger than these methods can create. Furthermore, no tests with oscillating vanes have been conducted at Reynolds numbers on the lower side of the MAV regime. As the point of this effort was not to create an entire review paper based on such designs, the reader is encouraged to review the 
following references, as only minor design changes have been observed between them: [51] through [62].

\subsubsection{Plunging and Pitching Wings to Mimic Gusts}

Another common practice for the creation of gust-like flows is to use wing maneuvers, e.g. pitching or plunging wings. The idea behind this assumes it is possible to recreate the relative flow by moving the model instead of moving the fluid. The validity of that assumption for steady experiments is settled but remains a topic of discussion in the research community for unsteady experiments. While some work has been done to validate the assumption, which will be discussed later, it is understudied as it is both costly and time consuming to test both moving fluid and moving models for potentially the same result.

The experimental examination of low Reynolds number unsteady flows and flows around oscillating/moving bodies are mostly performed in water channels or tow tanks rather than wind tunnels. This is primarily due to the fact that it is much easier to obtain forces and the desired Reynolds number in water as opposed to air. Granlund et al. showed that an oscillating freestream like that created by the oscillating vane designs presented earlier was in close agreement with the sinusoidal plunge of an airfoil in a water tank [63], suggesting the use of this method as a potential way to model gusts with simpler experimental apparatuses.

Other work by Carta compared a dynamically pitching wing to a plunging wing [64]. For these experiments, a wind tunnel was used and operated at $30.5 \mathrm{~m} / \mathrm{s}$ and 61 $\mathrm{m} / \mathrm{s}$. With their wing model having a 6-inch chord, the resulting Reynolds numbers was between $\sim 300,000$ and 600,000. The work showed that for small amplitude pitch at pre- 
stall conditions, there was a fairly decent correlation between the two data sets. However, as the airfoil entered the transition regions by increasing the amplitude of the maneuver, the correlation began to break down. Once large amplitudes were reached, the correlation had all but vanished.

Suada et al. were able to show that the measured fluid dynamic forces on a sinusoidally plunging wing were greater than those estimated by quasi-steady analysis [65]. This highlights the need to extend numerical estimations of such a problem beyond linear cases, as is commonly done. If a plunging wing is not well-estimated by quasi-steady analysis, surely a wing in a transverse flow will not be as well.

Shyy et al. outlined the importance of plunging and pitching wings in respect to modeling natural flyers [66]. It is known that these animals have the ability to passively reject gusts [67]. Therefore, the hypothesis has been presented that if one can model their wing motions as a simple plunge or pitch, it may be possible to model a gust's effects the same way. Furthermore, in a separate paper, Shyy et al. presents the basis of a computational model that was used to simulate the flow field for these motions [68]. Wokeock and Radespiel also made a similar comparison between experimental and numerical studies of pitching and plunging wing that showed good correlation to the experimental results [69].

Baik et al. studied a range of plunge amplitudes and frequencies and varied the effective angle of attack from -6 to 22 degrees in order to understand and track the Leading-Edge Vortex (LEV) being shed by such maneuvers [70]. Further discussion was had over the importance of this LEV in the creation of the high spike in lift seen 
in such maneuvers. This vortex and lift spike have been shown to be present in a gustwing interaction as well [71].

A potentially similar model motion to plunging is the pitching motion of a wing. In previous gust generating studies, specifically those created by oscillating vanes, a gust encounter was measured by the change in angle of attack the wing sees. This presents the hypothesis that a gust can be seen as an increase in effective angle of attack on a wing and the reason an increase in lift and eventual flow separation is shown. Thus, it makes sense that some have analyzed a pitching motion in regard to unsteady flows and gust encounters. One such work by Stevens and Babinsky examines both the flow structure itself as well as the forces and moments generated by such a motion [72]. Their pitch rig allows motion about the leading edge of the wing as well as the mid chord of the wing. Experiments were conducted in a water tow tank at Reynolds number of 10,000 . The lift spike that occurred at the beginning of the pitch motion was explored and the LEV's contribution to this spike explained. It was shown that having the pitching axis at the front of the wing produced a $20 \%$ higher lift spike than pitching about a point further back. Furthermore, a force model was produced and compared to the collected data. The model was shown to more closely match leading edge pitching than mid chord pitching.

Finally, Perrotta and Jones compared gust-like maneuvers, that is moving the wing opposite to the notional gust velocity, to a wing's interaction with a gust from a gust generator [71]. The gust generator developed by Perrotta and Jones will be discussed in the next section of this report, but the data collected from it was compared with various plunge maneuvers. The ratio of plunge distance versus distance traveled 
created various gust-like profiles that were thought to mimic various gust strengths and widths. Pitching maneuvers were also studied. The results showed that plunge and pitch maneuvers over predicted the lift coefficients measured by the wing in their vertical gust. Perrotta and Jones deemed that part of this over-prediction was an added mass effect, but there were still other forces at play. They concluded that it was not entirely possible to predict this type of gust encounter with a simple plunge or pitch of a wing. This result makes sense as a pitch and plunge motion should produce more lift than a simple sine squared gust profile. Pitch and plunge maneuvers will affect the entire wing chord simultaneously, whereas a gust encounter will only affect the portion of the wing that is currently in a gust. The results of Perrotta and Jones suggest that linear pitch and plunge maneuvers likely cannot recreate a gust interaction with a sine squared gust, but it is currently unknown whether other gust profiles may be recreated with these maneuvers.

\subsubsection{Vertical Gust Generators in Water Tunnels and Tow Tanks}

The use of water tow tanks allows for creation of certain unique types of gusts. By towing the test subject through a medium, there is less concern about blockage than there is in a wind tunnel. Furthermore, it is possible to move a wing into a gust which would not be acting on the wing at the start of its motion, while in a wind tunnel, a gust must be actuated to impinge on a static wing. Perrotta and Jones were able to use this to develop a novel gust generator in a tow tank through which a wing is pulled [73]. They constructed a generator that pumps a jet of fluid vertically from the bottom of a tow tank and towed a flat plate across it, as illustrated in Figure 13. Experiments were conducted at Reynolds numbers of 10,000, 20,000, and 40,000 with the plate held at 
angles of attack of 5, 10, 15, 20, and 45 degrees and constant gust velocity. Various tow velocities were used to obtain varying gust ratios. The gust ratio is defined as the vertical speed seen by the wing divided by the wing's tow velocity (or freestream flow), $\frac{V}{U_{\infty}}$. Wing-fixed time averaged Particle Image Velocimetry (PIV) was used to capture the flow field and forces were measured for all cases. A picture of these PIV results can be seen in Figure 14. The flat plate, having a chord of $5 \mathrm{~cm}$, was positioned so it would pass over the gust $15 \mathrm{~cm}$ above the gust nozzle. At this location, the stationary gust had a span of $16 \mathrm{~cm}$ with a peak velocity of $0.34 \mathrm{~m} / \mathrm{s}$. It was shown that as the wing entered the gust, a large LEV was produced creating a sharp, but temporary, increase in lift. Interestingly, as the trailing edge vortex (TEV) forms, a negative lift spike was generated for a brief period. When compared to traditional numerical models, such as Küssner's function, it was shown that the initial force from the gust matched closely, but the transient forces after the gust peak did not.

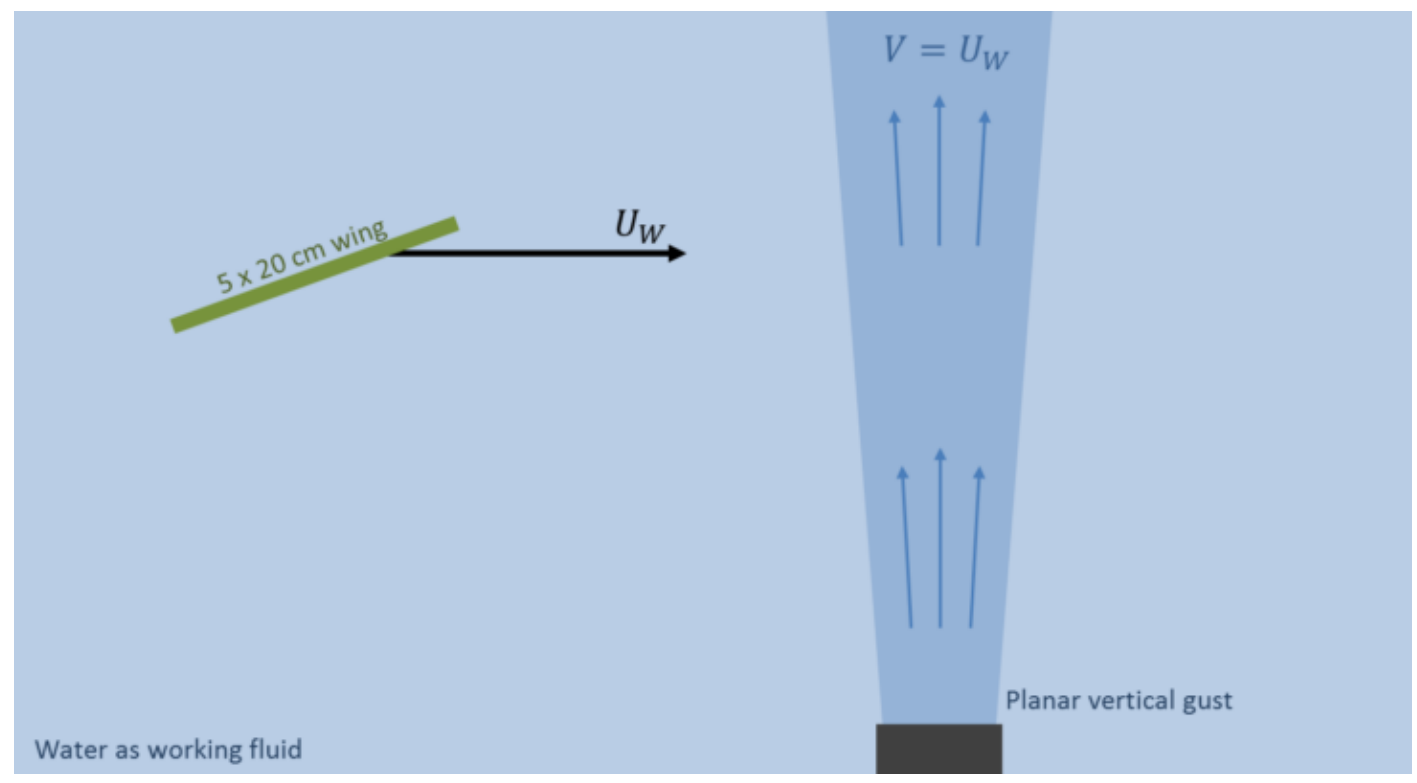

Figure 13: Sketch of Perrotta's flow from gust generator [73]. 


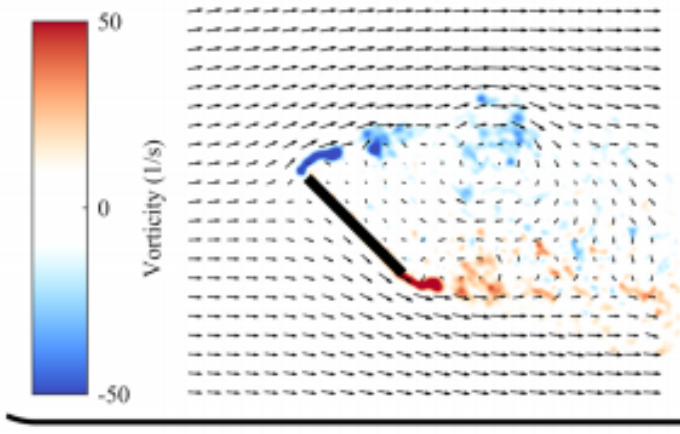

(a) $t^{*}=-2$

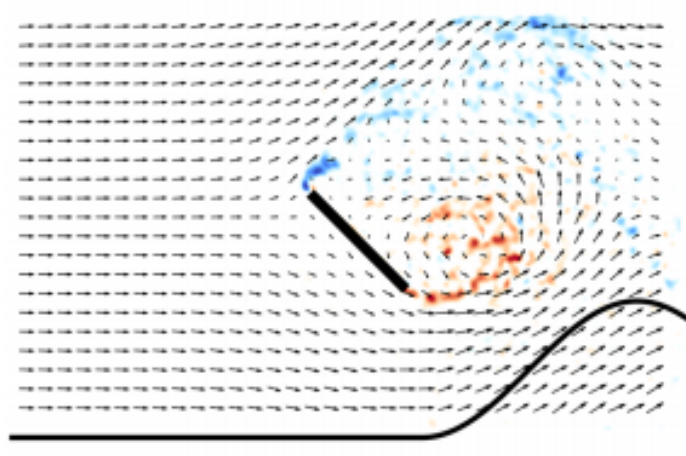

(c) $t^{*}=4$

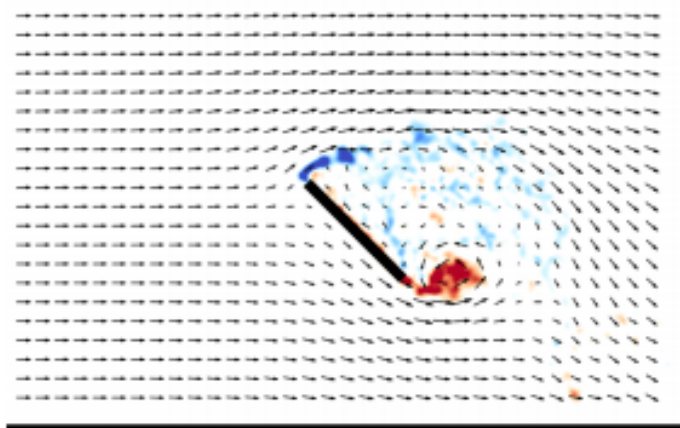

(e) $t^{*}=10$

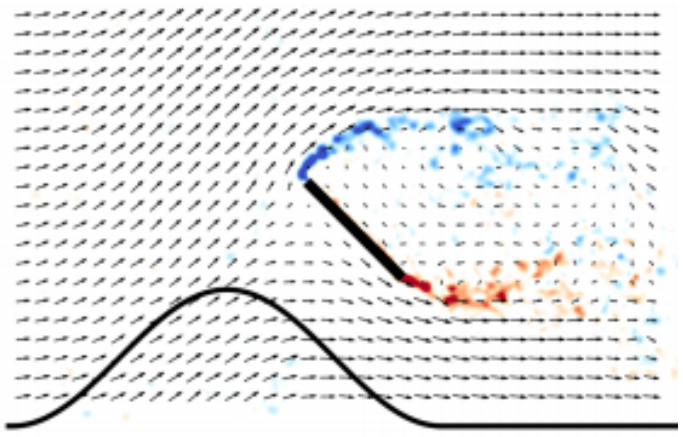

(b) $t^{*}=1$

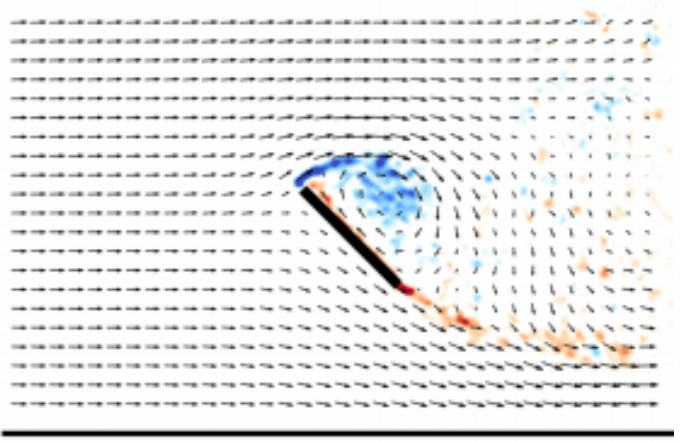

(d) $t^{*}=7$

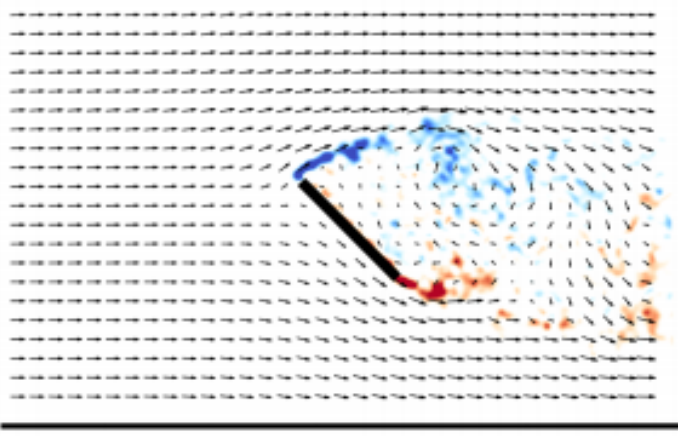

(f) $t^{*}=13$

Figure 14: PIV results of a wing entering a gust from Perrotta and Jones [73].

Corkery et al. used a similar approach in a tow tank with hopes of creating a tophat shaped gust [74]. Creating a top-hat shaped gust is important for comparison with many linear theories, such as Küssner's, which will be discussed later in this chapter. The design of this top-hat shaped gust is shown in Figure 15. Water was pumped across a tow tank and it was collected in an inlet that sucks in the flow expelled. This setup was shown to help minimize the diffusion that would occur without this inlet side. 
Initial results from this generator showed good correlation with the desired top-hat shape as well as good correlation to the predicted values from Küssner's function.

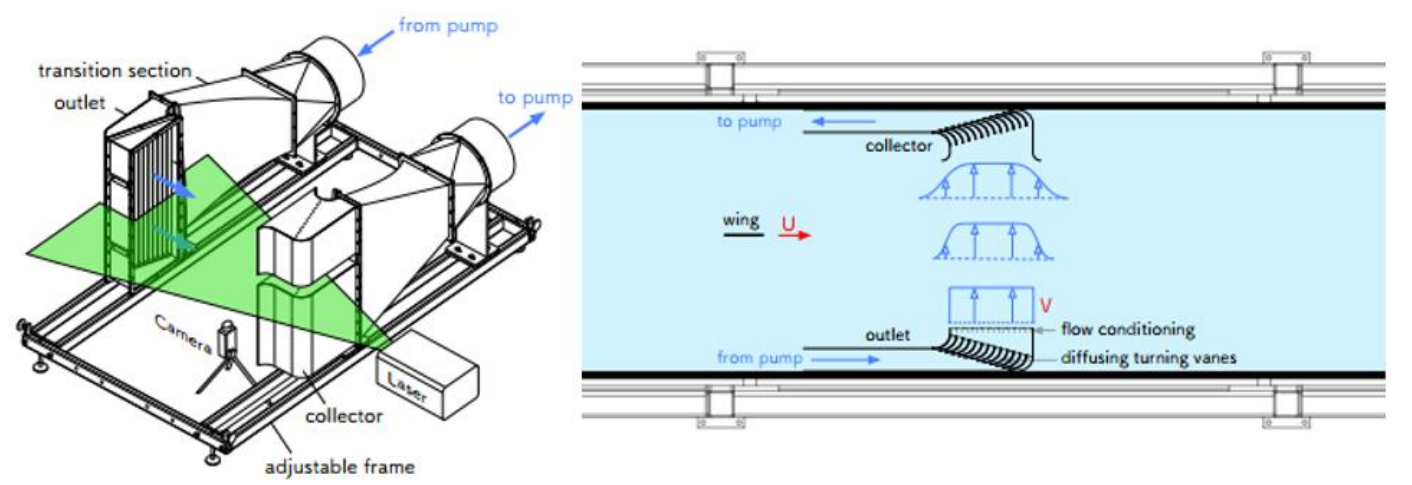

Figure 15: Top-hat gust generator design [74].

Biler et al. revised the design of the generator created by Perrotta and Jones to create a more controllable and wider gust generator [75]. The updated generator can be seen in Figure 16. The data collected with the revised generator was compared to results from a computational fluid dynamics (CFD) study by Badrya [75]. Several pump speeds were tested creating various gust velocities; however, the Reynolds number was held constant at 40,000. Based on the angle at which the flat plate entered the gust and the gust ratio $\left(\frac{V}{U_{\infty}}\right)$, the flat plate experienced various effective angles of attack with respect to the leading edge of the plate. Similar effective angles of attack, produced by different combinations of geometric angle and gust ratio, were compared to each other. The work concluded that there was a correlation between the effective angle of attack the plate saw and the peak lift produced. The experiments were then used to validate a computational method designed to simulate gust wing interactions. A novel approach to Field Velocity Method (FVM) was taken, a source term was added to classical FVM to account for gust wing interaction and showed close quantitative agreement with the experimental results. 


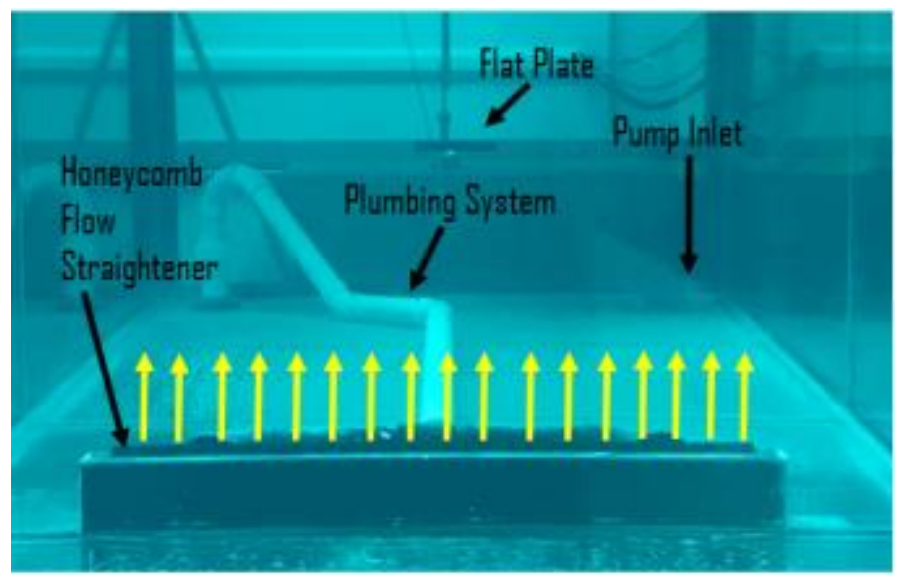

Figure 16: Biler et al. ’s gust design [75].

While these techniques have been shown to consistently generate large transverse gusts at MAV scales, the current effort focuses on a solution that could be used in air. Testing in air may allow for the possibility of testing an actual MAV in the created gust, a feat that would otherwise require significant waterproofing if done in water. These experiments also lack a way to obtain a step function gust with steady state postgust results around a wing. Wings in these studies only interact with a gust for a few chord lengths of travel, while it is quite possible that an MAV will see a gust that lasts many chords of travel. It can also be noted, by comparing Corkery et al.'s work to the other two works, that the community is still not sure if a sine squared or Top-hat shape gust is best. It is even quite possible that both gusts can occur in nature and that an MAV scale interaction must be studied in both cases.

\subsubsection{Computational Methods for Gusts}

Numerical examination of a gust is a crucial tool in future gust work as shown by Biler et al [75]. Yi et al. compared wind tunnel test data they collected to a structural numerical model they developed [54]. A flexible tapered wing was tested. Its root chord was $0.263 \mathrm{~m}$ and its tip chord was $0.07 \mathrm{~m}$. The tunnel was run from $20 \mathrm{~m} / \mathrm{s}$ until flutter 
could be analyzed at $50 \mathrm{~m} / \mathrm{s}$; equating to Reynolds numbers of $\sim 90,000$ to $\sim 900,000$ depending on speed and location on wing. The gust generating device used was an oscillating vane setup. Tip deflection of the wing under these gust loads was measured and compared to their model. "Fair to good quantitative agreements" were found between the two.

Golubev and Visbal created a computational fluid dynamics (CFD) study that simulated 3 types of gusts: time-harmonic gust, sharp edge gust, and Taylor Vortex [76]. For all cases, $M_{\infty}=0.1$ and the Reynolds number was 10,000 . Each set of results was compared to the others as well as a data from a pitching airfoil with a similar induced angle of attack. In doing so, they operated under the assumption that on some level, these gusts are comparable to that of a pitching airfoil. The comparison revealed the importance of both viscous and inviscid forces in modeling such gust phenomena and that there is in fact a difference between a pitch and these gust encounters as other works have concluded.

Golubev et al. presented an Implicit Large Eddy Simulation (LES) model that captured the flow on a NACA 0012 wing being affected by a vortical flow observed in oscillating vanes experiments [77]. Results were shown for a Reynolds number of 60,000 . They went on to explore the impacts of the remnants of the diffuse vortex crossing above versus below the wing after it is shed from two separate positions upstream.

Another notable computational study was presented by Zaide and Raveh, who examined gust effects on an arbitrary shaped wing with Mach numbers ranging from 0.11 to 0.7 [78]. Indicial responses and responses to a sharp-edge gust were computed 
for NACA0004, NACA0006, and NACA0012 airfoils. The reduced-order models predicted the lift and pitching moment histories accurately throughout the subsonic and transonic regimes.

Yang and Obayashi studied gust effects numerically at supersonic conditions, specifically Mach 2 [79]. They used a Navier-Stokes based solver and a Reynolds number of $27.5 \times 10^{6}$ for their computations. A gust of $50 \mathrm{ft} / \mathrm{s}(15.24 \mathrm{~m} / \mathrm{s})$ was simulated and shown to have very little impact on the flight of the aircraft.

Lancelot et al. also compared experimental results to a high fidelity numerical study [53]. The typical oscillating vane set up was used to create the gust in the experimental case. The tunnel, and matching CFD, were set for 15,21 , and $25 \mathrm{~m} / \mathrm{s}$. The vanes used were placed at the end of an open return wind tunnel and the gust was propagated into an open room. A hotwire placed in the gust was used to collect data. This data was then compared to that of a CFD simulation with very good correlation.

While computational studies are key to the fluid dynamics field, many of these CFD models cannot be benchmarked against experimentally measured gusts or gustwing interactions. This will continue to cast doubt on their validity until such experimental data is acquired.

\subsection{Analytical Models}

Wagner's indicial function of lift has long been used to predict the forces experienced by a flat plate in transient pitch maneuvers [80]. Wagner numerically modeled the lift generated from a wing abruptly accelerated to a constant streamwise velocity, in doing so defining the well-known Wagner-function. For an airfoil at a constant angle of attack, the lift starts at 50\% of the steady lift and asymptotically 
approaches the steady lift. This is due to the creation of a vortex that is shed after the airfoil starts moving. As the airfoil moves away from the initial location, the bound circulation gradually increases towards the value at steady lift.

Similarly, Küssner's function of lift has been used as a representation for a wing entering a spatially varying gust [80]. Küssner developed this function to show how lift changes as a function of time in a gust. The method and assumptions used are similar to Wagner's function. Küssner separated his airfoil into chordwise sections for the analysis done and showed how each section of the airfoil would experience the gust at various times. Thus, it was concluded that an airfoil entering a gust behaves differently than a wing encountering an abrupt start motion. The key difference is that Küssner's model allows different incoming flow angles while Wagner's is fixed across the entire wing.

Theodorsen used a 2D elementary flow solution to Laplace's equation to develop the velocity potential functions for a pitching and plunging flat plate with a flap [81]. Theodorsen assumed small perturbations, prescribing a flat wake behind the airfoil extending to infinity. The airfoil motion was also required to be harmonic. This assumption allowed the vortex sheet extending from the trailing edge to infinity to be integrated, leading to a solution in the form of Bessel functions. Through this solution Theodorsen showed that the lift due to circulation was a function of the reduced frequency, $k=\frac{\omega \frac{c}{2}}{V}$, where $\mathrm{c}$ is chord of the wing, $\mathrm{V}$ is flow velocity, and $\omega$ is pitch frequency. This reduced frequency is an important parameter in the pitching or plunging wing problem. Therefore, if it is shown that the created gust can be modeled as such, it will be possible to extract the gust's reduced frequency and possibly model 
it with Theodorsen's function. However, due to the harmonic condition required for the airfoil's motion, this comparison will only truly fit for gusts that are themselves harmonic, such as those developed by oscillating vanes.

Wagner, Küssner, and Theodorsen's results are the most widely used for modeling the flow of a wing through a transient gust. There are, however, serious limitations to these methods. First, all three models are based on flat plates, which simplifies the problem because all vorticity is generated from either the leading or trailing edge. Second, they over-simplify the gust by assuming sharp-edged gust encounters, while it is thought that real-world gusts have varied profiles. Finally, on some level, they all rely on small perturbations for the derivation of their respective theories, which at MAV scales has been shown to break down. These theories, while useful in some cases, would need to be adapted to show greater correlation to realworld, experimentally testable, MAV gust scenarios.

\subsection{Scope of Present Research}

While prior work has shown that the creation of gusts in controlled environments is possible, the previous systems all lack at least one key feature the MAV-gust interaction community requires. The non-linearity and unanticipated behavior of airfoils at low Reynolds numbers remains a barrier for the use of higher Re data for MAV design. The use of oscillating vanes in a wind tunnel lacks the ability to create gusts at the test article that last longer than a brief period. Furthermore, it has not been shown that vanes are able to create the desired gust magnitudes required for MAV scales. Experiments in water have been shown to be effective, but the current effort aims to design an apparatus that would perform just as well, if not better, in air. This 
study also wants to create a moving fluid experiment as opposed to a moving model, as moving models have been studied fairly extensively. Finally, there is need for further data with which to validate computational solutions, and thus the experiments needed to be carried out in a physical space, not a computational one.

The current research highlights the design and development of a wind tunnel gust generating system. The proposed gust-generating system will be designed to create a vertical gust in constant freestream flow in a wind tunnel, similar to an updraft an MAV may encounter. The goal for this novel system was to create a gust ratio of between 25 and $40 \%$ of the freestream velocity. A fully functional innovative gust generator will be characterized for MAV scale gusts. This study will show the feasibility of the proposed vertical gust generator for testing real world gusts in a controlled laboratory setting. Since there are assumed to be a variety of gust profiles seen in nature, it was decided to isolate one flight scenario. The ideal profile was set as a gust seen by an MAV hovering near the edge of a building as presented by White et al. [82]. As a horizontal flow approaches a building, it is thought to bend around the top of the building, producing both an updraft and an increase in horizontal flow. This idea is portrayed in Figure 17. If the gust generator developed here is better at creating this type of profile than previous attempts and matches closely with what would be expected in real world scenarios as hypothesized, the door will be open to more complete testing of various models to help determine the aerodynamic effects of gusts on MAV scale wings. 


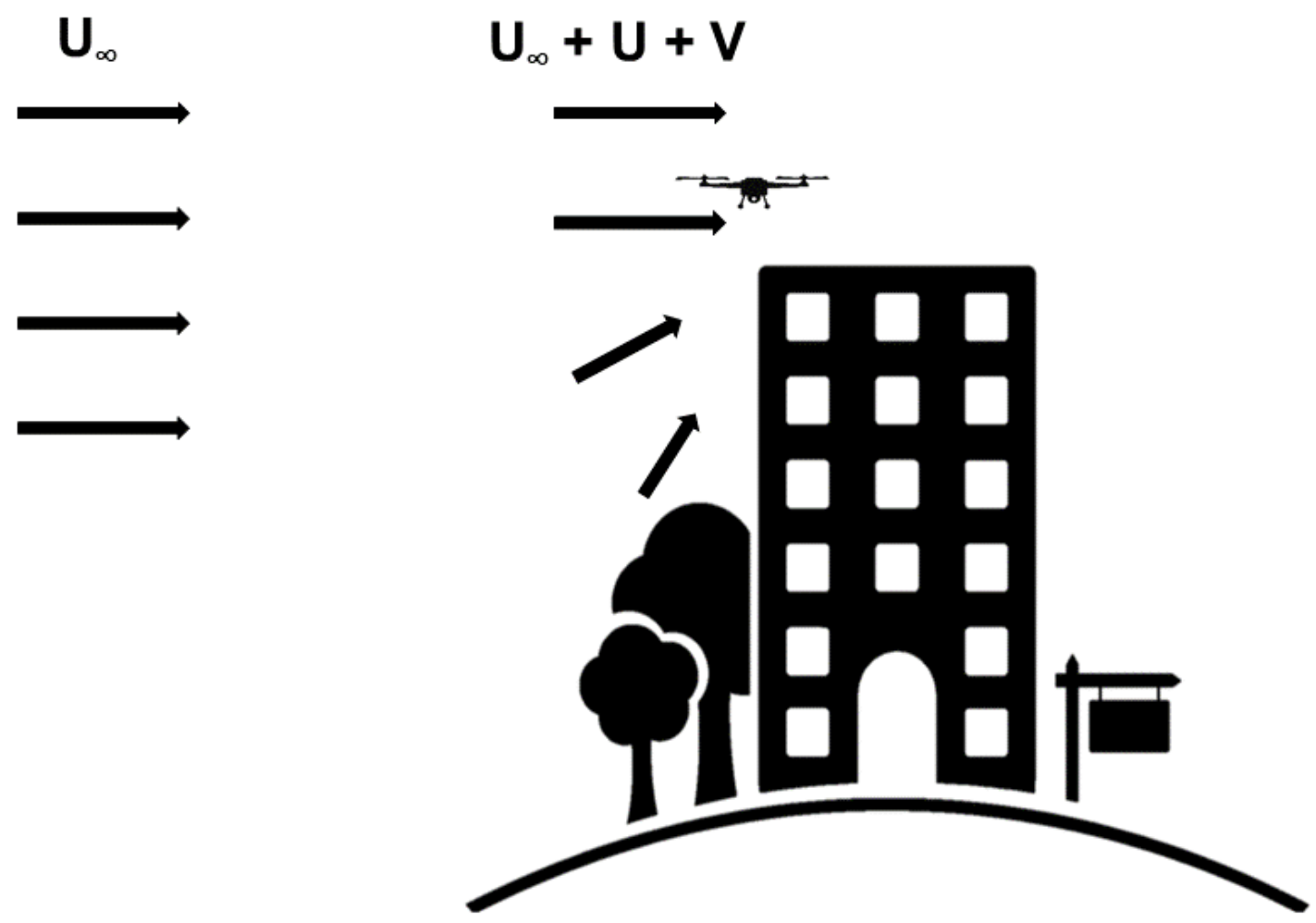

Figure 17: Vertical flow near top of building.

Additionally, the current study will briefly touch on the fundamental aerodynamics question of whether experimental gusts in air and water are quantitatively the same. This is highly relevant to experimental MAV research because the low Reynolds numbers that characterize MAV scales are more easily attained in water while the vehicles maneuver in air. This development of a system specifically tailored to airfoil sections at MAV scales in air will hopefully allow scientists and engineers to extend understanding beyond the linear theories, which rely on the assumptions of attached flow and small perturbations to a regime governed by large disturbances, flow separation, and the growth of large coherent vortical structures. 


\section{Chapter 3: Experimental Setup}

\subsection{Experimental Facility}

\subsubsection{Wind Tunnel}

The experiments presented here were conducted in the Microsystem Aeromechanics Wind Tunnel (MAWT) facility; a closed loop Aerolab wind tunnel at the US Army Research Laboratory (ARL), shown in Figure 18. This wind tunnel has a $3 \times 3 \times 6 \mathrm{ft}(0.91 \times 0.91 \times 1.82 \mathrm{~m})$ test section and is capable of operating between speeds of $1 \mathrm{~m} / \mathrm{s}$ and $100 \mathrm{~m} / \mathrm{s}$ with measured turbulence intensities of $0.1-0.3 \%$ depending on flow speed.

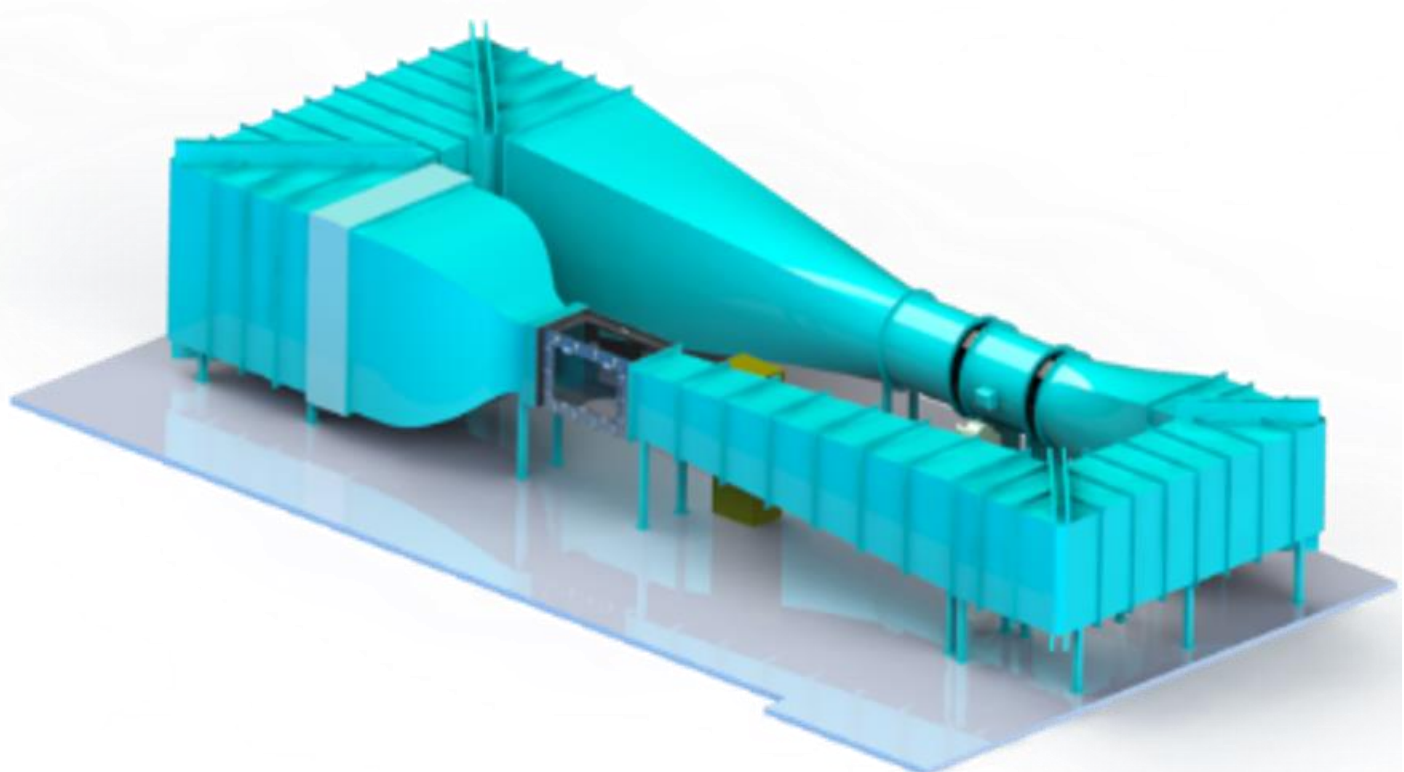

Figure 18: 3D model of the MAWT Facility.

An aluminum frame mounted to a traverse system, not shown in Figure 18, surrounds the test section and is intended for easy change of spanwise measurement location for the Particle Image Velocimetry (PIV) system, which is mounted to the outside of the traverse frame. The ceiling and walls of the test section are highly 
reconfigurable and allow for creation of custom acrylic or medium density fiberboard (MDF) inserts. The floor of the test section is mostly aluminum but has a 30-inch wide removable base for a traditional yawing test setup. An already existing experimental setup can be used to mount a full-span wing model at the mid-height of the tunnel test section. Wing pitch angle was controlled by a Galil 4020 servo controller which was connected to a servo motor with an attached 4000 counts per revolution encoder. A simple PID control system built into the Galil software was used to control the wing angle. The Galil Controller can provide servo update rates as high as $32 \mathrm{kHz}$, and processes commands as fast as 40 microseconds [83]. A physical target attached to the wing was used to level the wing to $0^{\circ}$ incidence angle using the PIV camera. The error associated with wing placement was calculated to be $0.20^{\circ}$ using the pixel accuracy of the target and camera scaling. The controller was effective at holding the wing angle of attack to the exact motor count prescribed. However, the resolution of the encoder introduced some uncertainty since the number of counts per degree is not a rational number $\left(1^{\circ}=11.111\right.$ counts). If that uncertainty is assumed to be 1 count $\left(0.09^{\circ}\right)$ and is then combined with the zeroing uncertainty, the total uncertainty in wing angle is approximately $0.22^{\circ}$. The gust actuation and the pitching motion were controlled through LabVIEW software while the tunnel itself was controlled with a control program designed for such a tunnel by Aerolab. For all tests, the tunnel fan was operated at 37.5 RPM, which was shown in previous calibrations to equate to a nominal tunnel speed of $1.5 \mathrm{~m} / \mathrm{s}$. 


\subsubsection{Wing and Wing Mount}

The wing presented in the results is a NACA 0012. The wing had a chord length of $0.12 \mathrm{~m}$ and spanned the entire width of the tunnel. This resulted in a gust-off Reynolds number of $\mathrm{Re}=12,000$ and a physical aspect ratio is noted as 7.62; although for experiments the aspect ratio was assumed to be infinite as it spanned the entire tunnel width. It should be noted that the activation of the gust generator could alter the flow in ways that will alter the Reynolds number. This effect is further discussed later in this report. The wing itself is made from machined aluminum coated with a black paint to smooth the surface and minimize reflections when used with the particle image velocimetry laser.

The wing mount can be seen in Figure 19. On both ends of the wing, holes were machined and tapped with threading. To install the wing, the outer wall is removed, and the wing was screwed into a threaded rod which attached to the Galil servo. The wing was then held in place with a stand as the opposite wall was reinstalled. A threaded rod was placed through the wall and screwed into the wing and the stand was removed. 


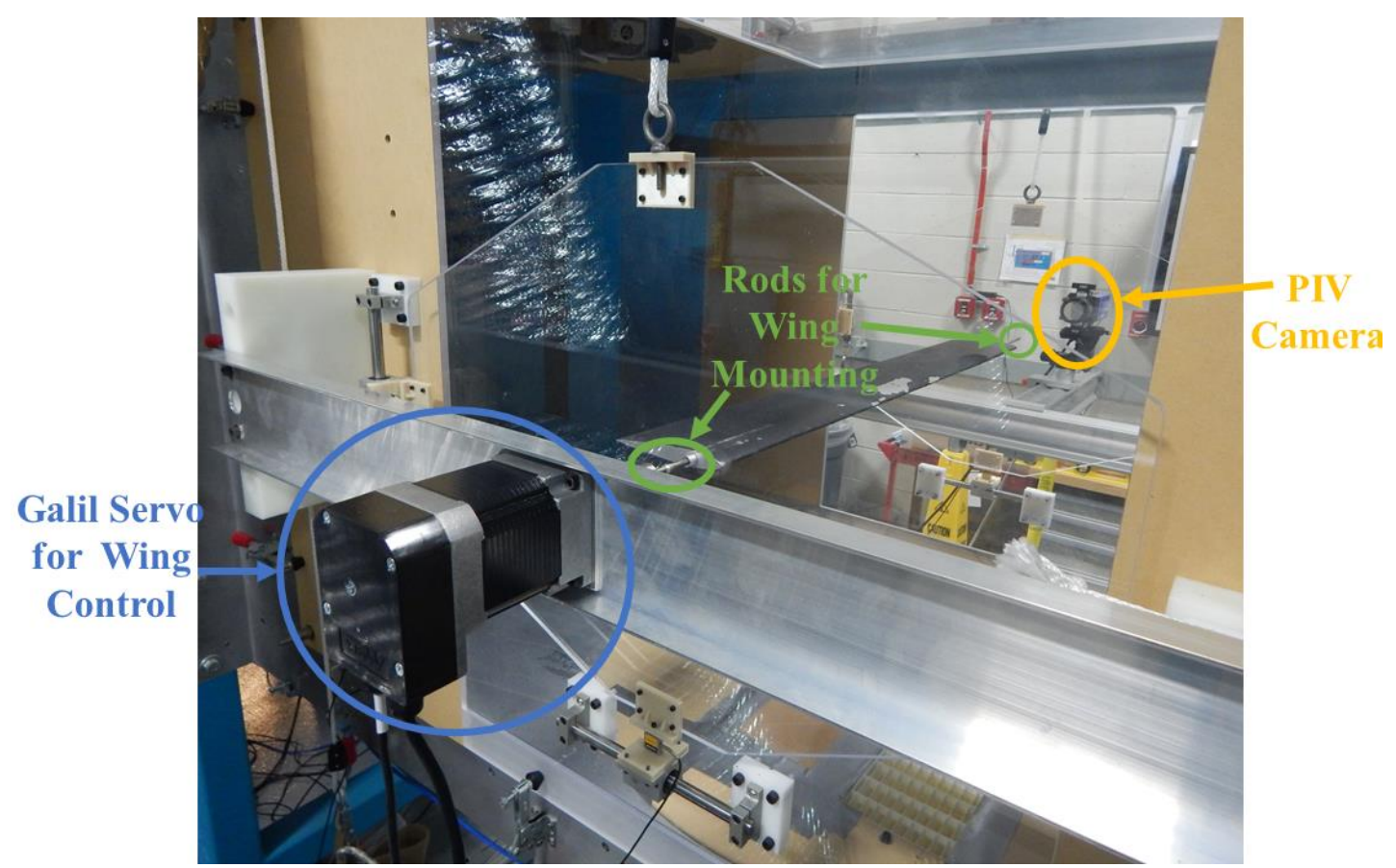

Figure 19: Wing mount setup.

\subsection{Gust Design}

\subsubsection{Gust Design Goals}

The initial conceptual design for the gust generator can be seen in Figure 20. The basic idea was to inject flow through the floor of the tunnel to simulate a transverse gust. It was desired to create a gust ratio, as defined by previous works as the ratio between the vertical velocity in the flow to the mean freestream velocity, $\frac{V}{U_{\infty}}$, greater than 0.3 with the gust generator. While this is not as large as some of the previously examined gust generators, this magnitude was selected as a proof of concept design criteria. There was also desire to minimize turbulence and maintain laminar flow both inside and outside of the gust as much as possible. Because the flow field of the gust was of primary interest, a closed return loop was desired to ensure PIV seeding particles were present in the gust as well as to conserve mass throughout the new wind tunnel- 
gust generator system. Finally, it was desired to have the ability to start and stop the gust as quickly as possible.

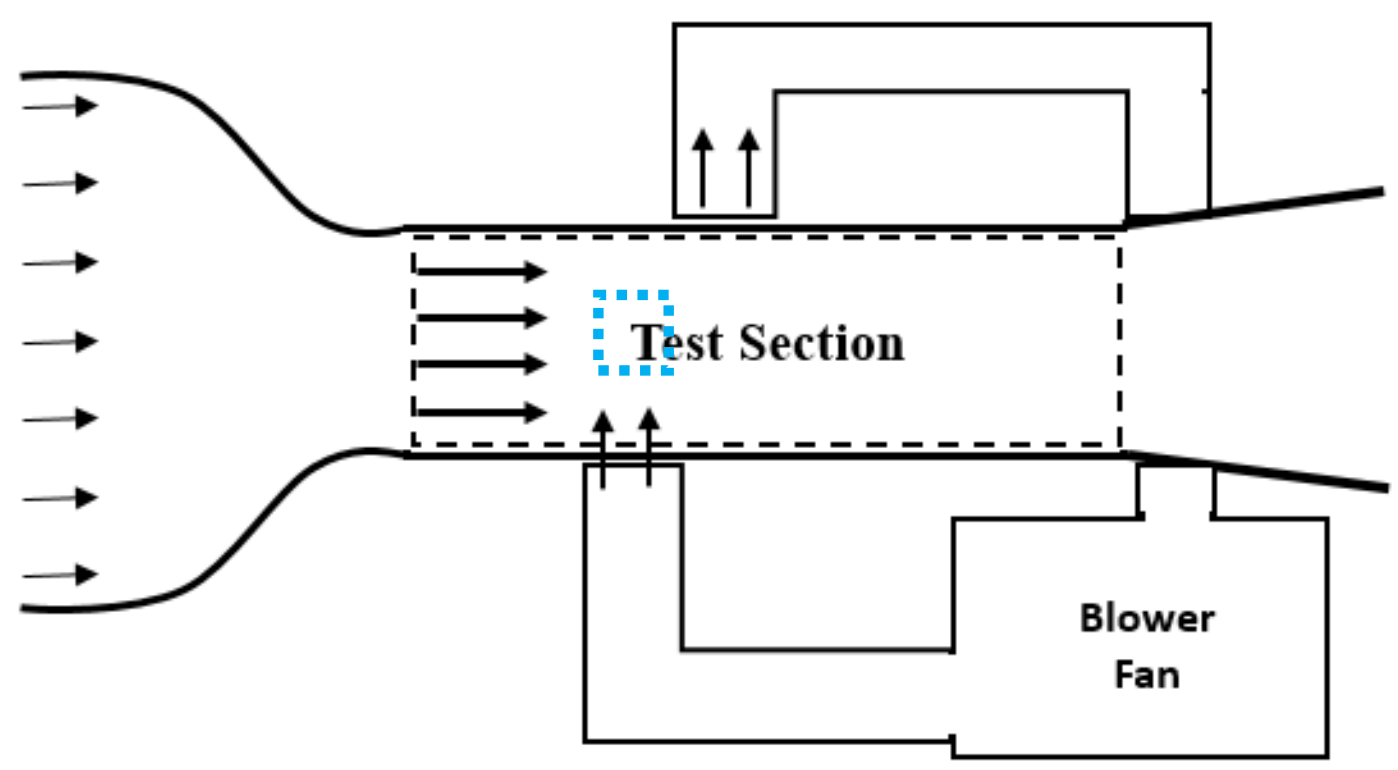

Figure 20: Initial sketch of gust generator.

Blue dashed box represents estimated PIV field of view.

\subsubsection{Fan Research and Selection}

It was found that the common metric used for comparing fan power is the cubic feet per minute ( $\mathrm{cfm}$ ) of air the fan could move. Therefore, to determine the best fan selection, it was first required to know what flow rate was desired. This was done using a simple mass flow rate equation:

$$
\dot{m}=\rho_{1} U_{1} A_{1}=\rho_{2} U_{2} A_{2}
$$

Since both mediums were assumed to be room temperature air, the density values would cancel and thus the flow rate would be entirely based on velocity of the flow and area it passes through. Thus, the jet size and strength at the bottom of the tunnel had to be determined in order to select a fan with the proper strength. 
As previously noted, the tunnel floor had a removable 30-inch diameter plate. This would be the main area the gust would duct through. The desire was stated to make the gust itself enter as a rectangle in the bottom of the tunnel in an attempt to make a sharp-edged gust. Furthermore, the gust needed to be as wide as possible in the spanwise direction to help minimize 3D effects. It was determined that the gust nozzle should minimally be $12 \mathrm{~cm}$ wide in the freestream flow direction to match the chord of the wing being tested. Finally, there was desire to place the gust both in front of and behind the wing to test the effect of gust location on wing performance. After exploring the geometry of the tunnel, it was determined that there was a maximum forward position the gust could go before decreasing the span of the gust significantly, (see Figure 21). This position was chosen as the maximum forward position and resulted in a subsequent gust span of $50.8 \mathrm{~cm}$, or $55 \%$ of the tunnel span. After this location was determined, the gust nozzle was designed to be 5 inches (1.06c) wide in the freestream flow direction. This was slightly larger than the wing chord which would hopefully increase the area over which the gust's effects could be seen, but not so large as to significantly slow the initial gust velocity coming from the nozzle.

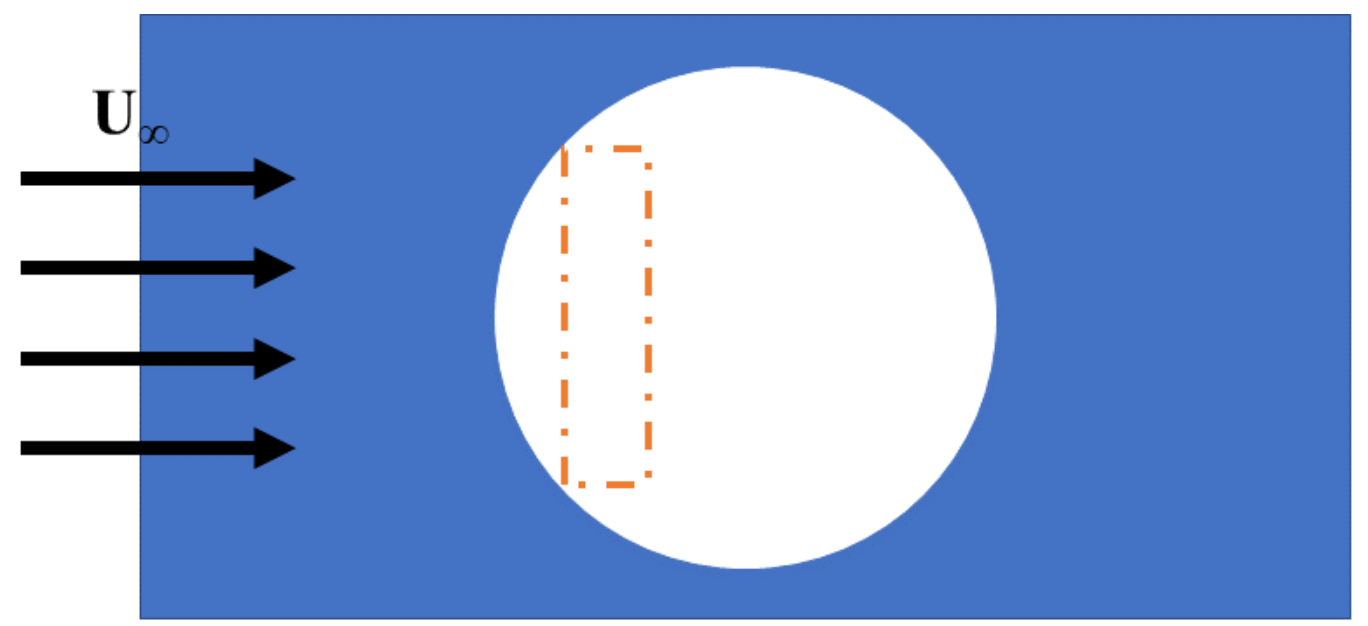

Figure 21: Top view example fan nozzle position and size. Image to scale. Orange dashed box: chosen size with forward position. 
The final selection criteria was the gust jet velocity. The wind tunnel freestream velocity was planned to be $1.5 \mathrm{~m} / \mathrm{s}$ to match other experiments being conducted at the MAWT facility. This speed was on the slower side of fixed wing MAV flight, but still a common speed for rotorcraft of this size and comparable to that of some animals. Furthermore, it would produce a Reynolds number small enough to compare to some water tank experiments, $\sim 12,000$. It was hypothesized that a gust nozzle exit velocity of 2 to 3 times that of the tunnel velocity would create the desired gust ratio at the test article. It was also assumed that losses from the ducting and gust nozzle itself would further drop the fan exit velocities by as much as $50 \%$. Thus, a goal was set to pick a fan that could produce speeds of around $10 \mathrm{~m} / \mathrm{s}$, or 1970 feet per minute. To achieve this, the selected fan would need to be able to produce flow rates of 1300 to $1400 \mathrm{cfm}$.

A fan was found and chosen that met all the desired requirements: a JD Manufacturing VES 161C, shown in Figure 22. This variable speed fan can produce up to 1440 cubic feet per minute flow rate and has a preinstalled plug. However, the two key features that made this fan so attractive for this project were the box frame and the front slats. The box frame made the installation of a larger fan casing and close loop ducting system easy to install. Furthermore, it was thought that the slats could be controlled to open and close as desired; functionally starting and stopping the fan's flow, and thus the gust. 

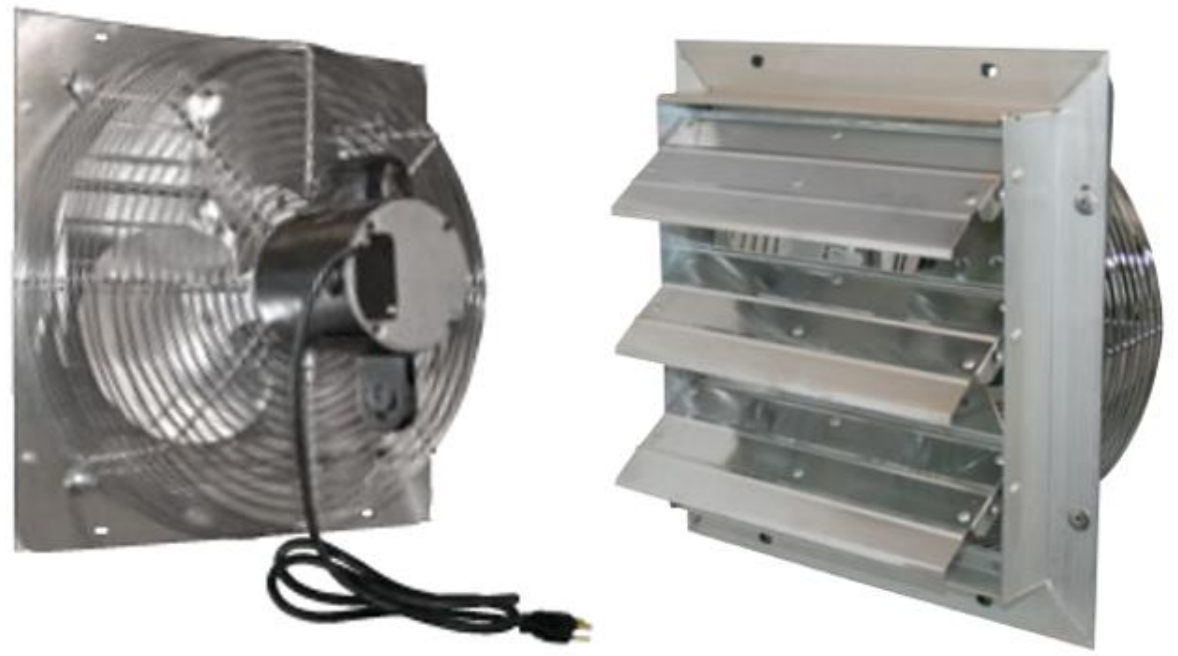

Figure 22: JD Manufacturing fan.

A Gino Development 09-0123 TruePower Rheostat was used to control the fan speed settings. Fan speeds selected were a full-power, mid-power, and low-power setting. The mid and low power speeds were selected arbitrarily and were not specifically set to an inlet jet speed.

\subsubsection{Final Design}

The gust generator was designed as a closed-loop, fan-driven jet that entered the wind tunnel from the bottom. Recirculating air was drawn from the ceiling downstream of the gust inlet. Gust intensity at the test model location was controlled by a variable speed fan connected to the rheostat device. A Galil 4000 counts per revolution servo motor controlled by the Galil 4020 controller was connected to the shutter system built into the front of the fan and was used to rapidly start and stop the gust. A fan housing was created out of plywood to help hold the fan stationary and create as much of an air tight fit as possible. A honeycomb flow straightener was added inside the fan housing to reduce turbulence levels. The honeycomb was made of aluminum, 4 inches thick, 
with 0.25 " holes. A contraction section made of cardboard connected the fan housing to a $25.4 \mathrm{~cm}$ flexible duct, which in turn was connected 90-degree aluminum elbow. This elbow connected to an expander that was created with ABSM30 on a Fortus $400 \mathrm{mc}$, which is a fused deposition modeling (FDM) type 3D printer. It had guide vanes on the inside to evenly spread the flow from the round duct to a rectangular floor inlet measuring $12.7 \mathrm{~cm}$ x $50.8 \mathrm{~cm}(1.06 \mathrm{c}$ x $4.23 \mathrm{c})$ and can be seen in Figure 23.

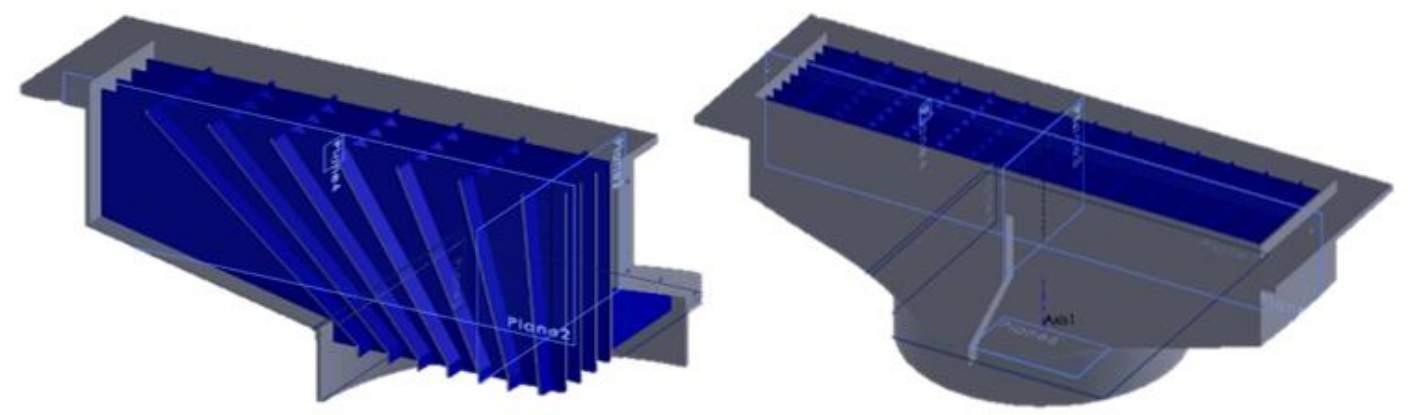

Figure 23: Gust nozzle.

This expander was attached to a cut pressboard circle that set into the hole at the bottom of the tunnel. The pressboard had an outer diameter of 32 inches with a smaller inner lip of 30 inches that fit snuggly into this hole. It did create a bump at the bottom of the tunnel of 0.25 " but was sanded to make this bump as smooth as possible to reduce any turbulence created. It was deemed to be far enough away from the wing as to not affect the flow at this location, a fact confirmed later during the flow characterization.

Due to the fact that the wing mount was at a fixed location in the tunnel, the gust was designed to be adjustable in both its power and position to optimize the interaction at the wing location. It was possible to position the gust inlet at four streamwise locations relative to the tunnel mid $\mathrm{x}$-axis location (also the quarter chord of the wing): $13 \mathrm{~cm}$ ahead (1.08c), $8.5 \mathrm{~cm}$ ahead (0.71c), $8.5 \mathrm{~cm}$ behind (0.71 $)$, and $13 \mathrm{~cm}$ behind (1.08c). These positions from farthest upstream to furthest downstream will be here on 
referred to as Position 1, Position 2, Position 3, and Position 4 respectively and can be seen in Figure 24.

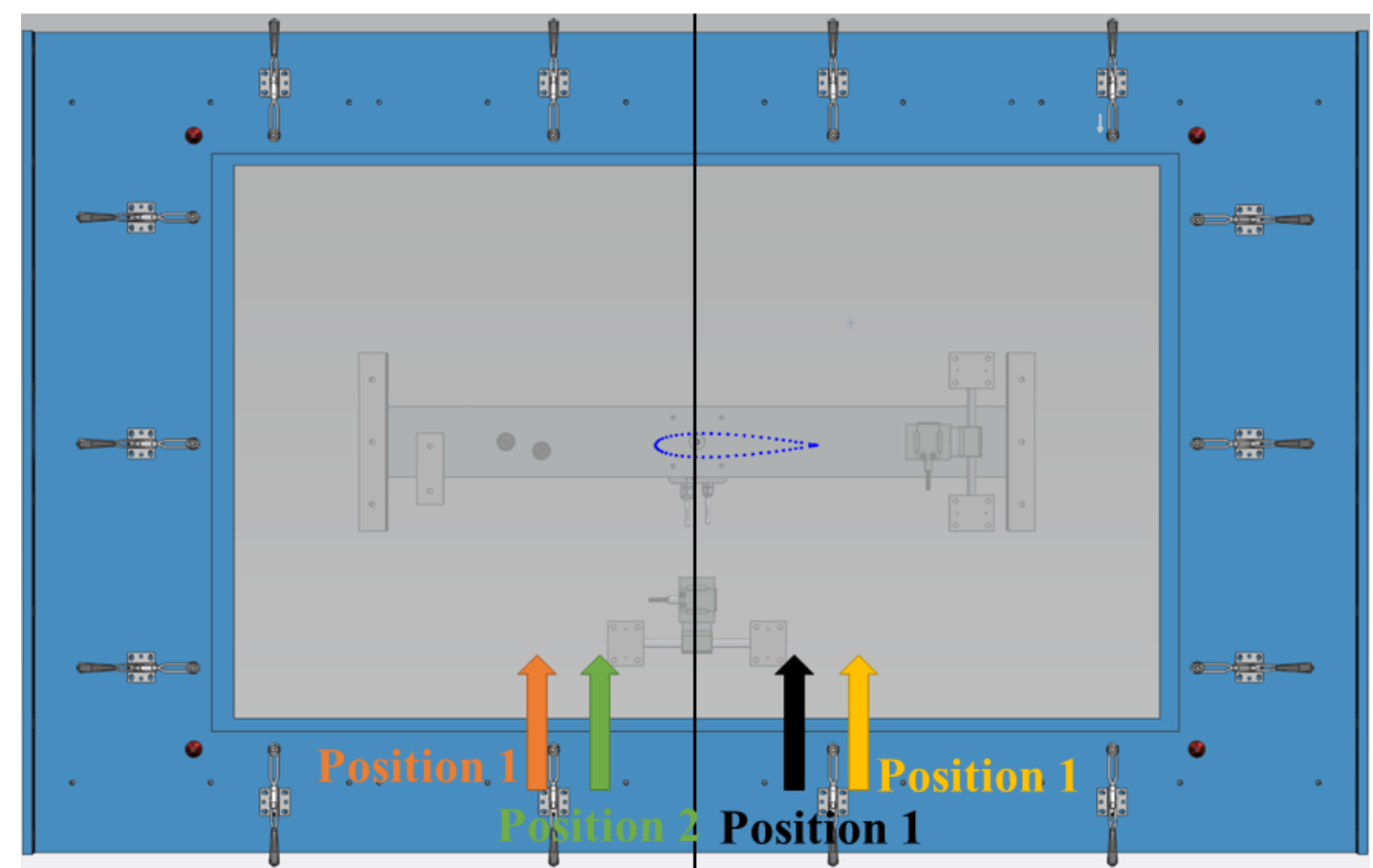

Figure 24: Gust entrance location on tunnel floor.

Figure 25 shows a 3D model of the final setup. The return duct was placed at the top of the tunnel, but as far downstream as possible to minimize effects seen by the wing. The center of this duct ended up being 30 inches $(6.35 \mathrm{c})$ downstream of the tunnel midpoint which is the same as the wing's quarter chord location. It was also made of a pressboard in the same style as the tunnel roof slats. It was approximately 16 inches in the chordwise direction with a 12-inch hole in it. Another flexible duct was attached to this hole and fed back through a cardboard expansion into the fan box mount. All connection points were sealed with duct tape to reduce leakage. 


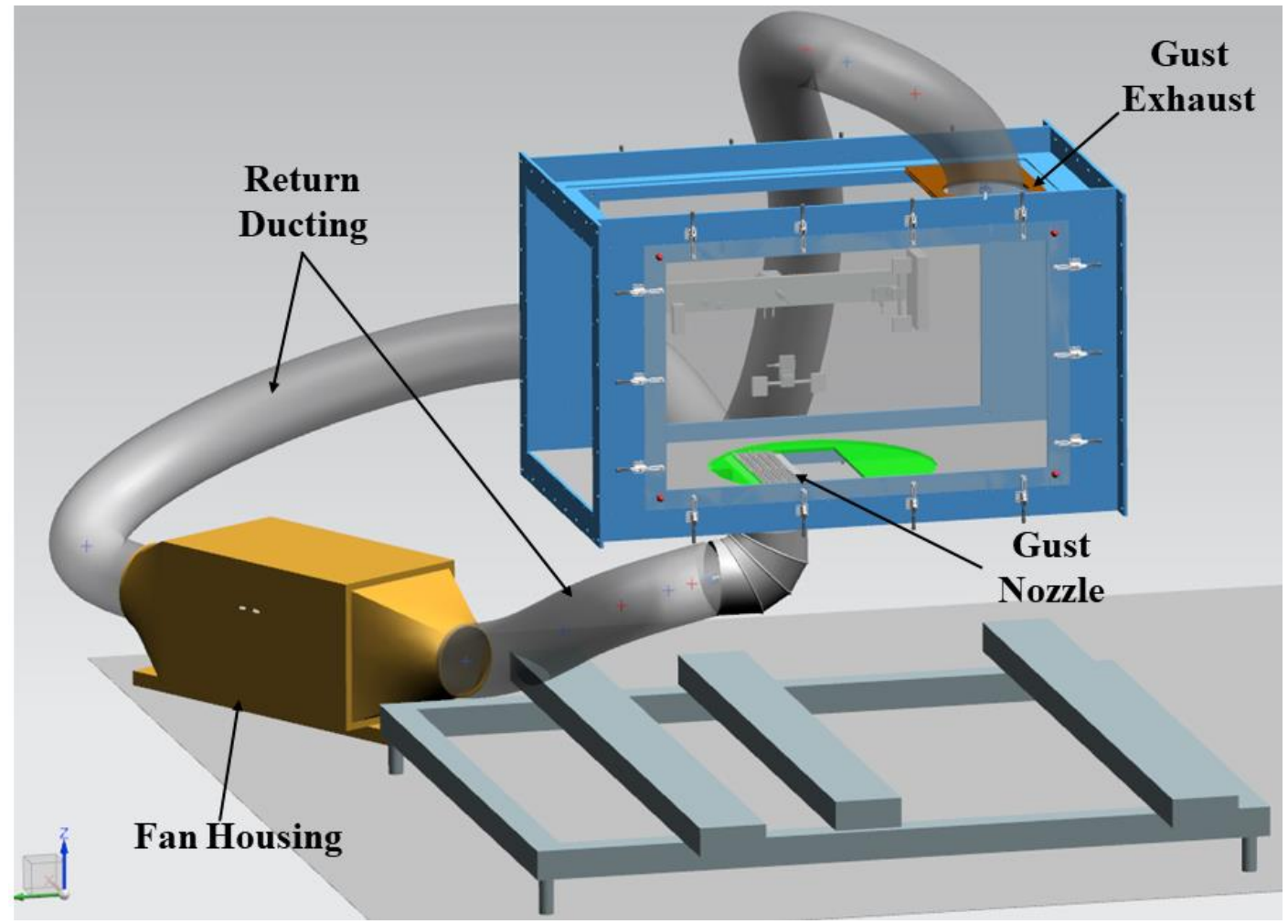

Figure 25: 3D model of final setup with wind tunnel missing.

\subsection{PIV: Particle Image Velocimetry}

The fluid motion was the primary interest of this study, so the data sets presented here are largely PIV results. PIV is a common measurement technique widely used for fluids research. As a brief review, PIV works by the firing of a high-speed laser that illuminates a seeding particle in the flow. The locations of these illuminated particles are captured on a high-speed camera in rapid succession. Regions of particles are mapped between images using image correlation techniques, which are in this case built into the LaVision DAVIS 8.4.0 software. Displacements from the image correlation are converted to velocity vectors using the known time between images. For a more in depth review of PIV, the author suggests a review paper by Prasad [84] and the textbooks by Adrian and Westerweel [85] and by Lourenco et al. [86]. 
PIV data was recorded using a LaVision Imager Pro X 4M (2112 × 2072 pix $)$ camera and a Litron Nano PIV laser illumination (532 nm). The seed particles used were atomized Di-Ethyl-Hexyl-Sebacat (DEHS) oil droplets that spread evenly throughout the flow. A $60 \mathrm{~mm}$ Nikon lens was used, and the resulting field of view was approximately $32.5 \times 32.5 \mathrm{~cm}$, which covered roughly the middle-third of the test section, Figure 26. Correlation was done using a multi-step correlation. A square $32 \times 32$ pixel window with no window overlap was used to pre-target a 24-pixel radius circular window with a $25 \%$ window overlap. Two passes were done with the smaller window and the process resulted in a vector spacing of approximately $3 \mathrm{~mm}$ between vectors. A $\Delta t$ between images of $150 \mu$ s was used for all tests unless otherwise specified. For all data, when vector fields are shown, every $6^{\text {th }}$ vector is displayed to declutter the images presented. 


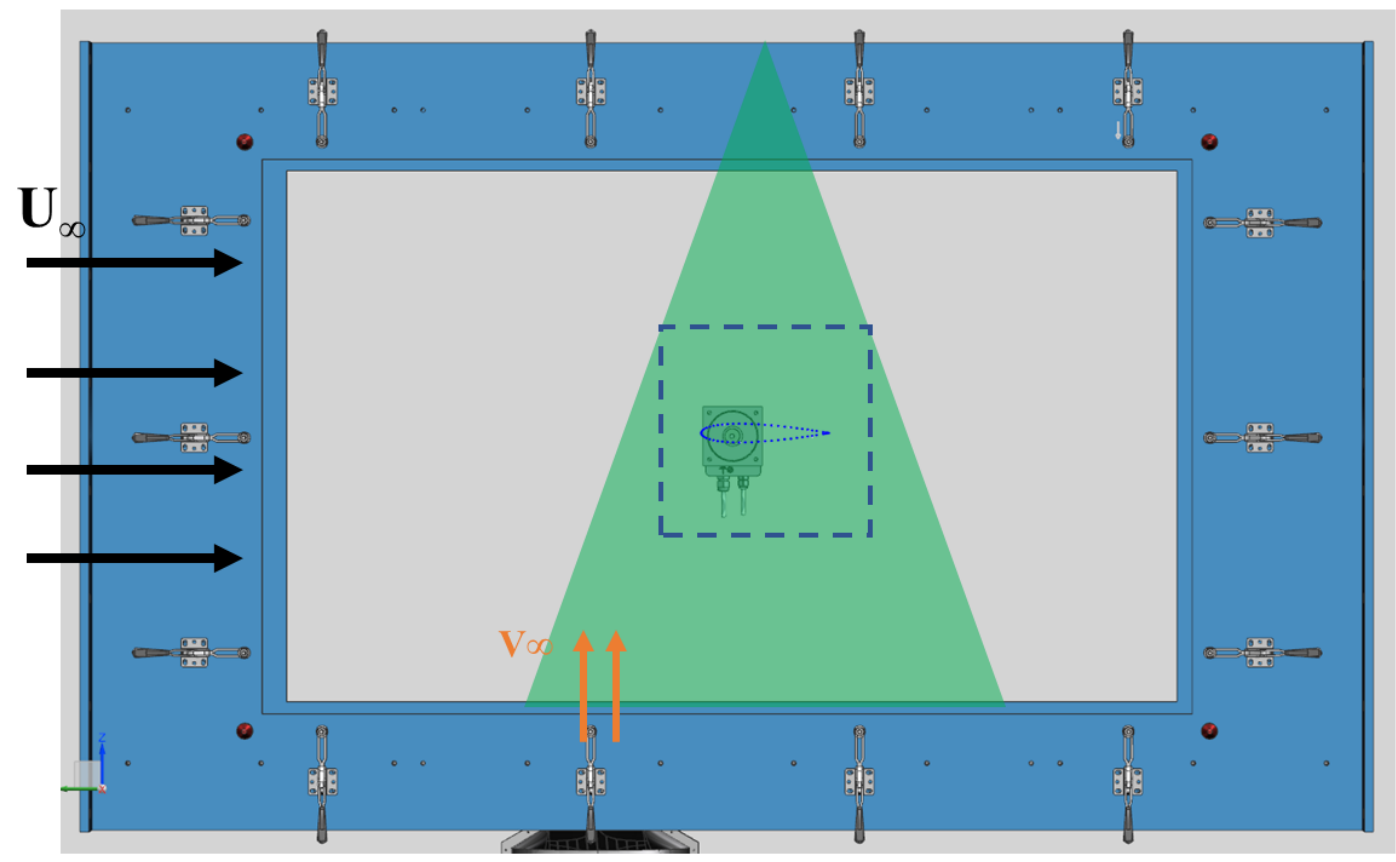

Figure 26: PIV field of view.

Side view of test section. The dashed box represents the field of view of PIV images. Black arrows indicate direction of the freestream flow and the orange arrows represent the location of the gust Position 1. Green triangle is a representation of the laser sheet. Wing location shown as dotted line.

\subsection{Tunnel Characterization}

Initial characterization was done to confirm that the installation of the gust generator did not affect the mean freestream flow generated by the wind tunnel. Confirmation had to be made that the tunnel could obtain the same set speed and that there was no alteration of background flow variation, measured here as root mean squared (RMS) velocity. The outlet side of the gust generator remained stationary for each test, however, the inlet jet location was moved, and the flow was measured at each of the 4 locations specified in the previous section. During characterization the tunnel was empty (i.e. no wing or other test article was in place) and was set to run at $1.5 \mathrm{~m} / \mathrm{s}$. Figure 27 shows the contour of the horizontal (U) velocity seen in the flow normalized 
by the expected tunnel freestream $(1.5 \mathrm{~m} / \mathrm{s})$. Figure 28 shows the contour of the vertical

(V) velocity in the flow normalized by the expected freestream velocity.

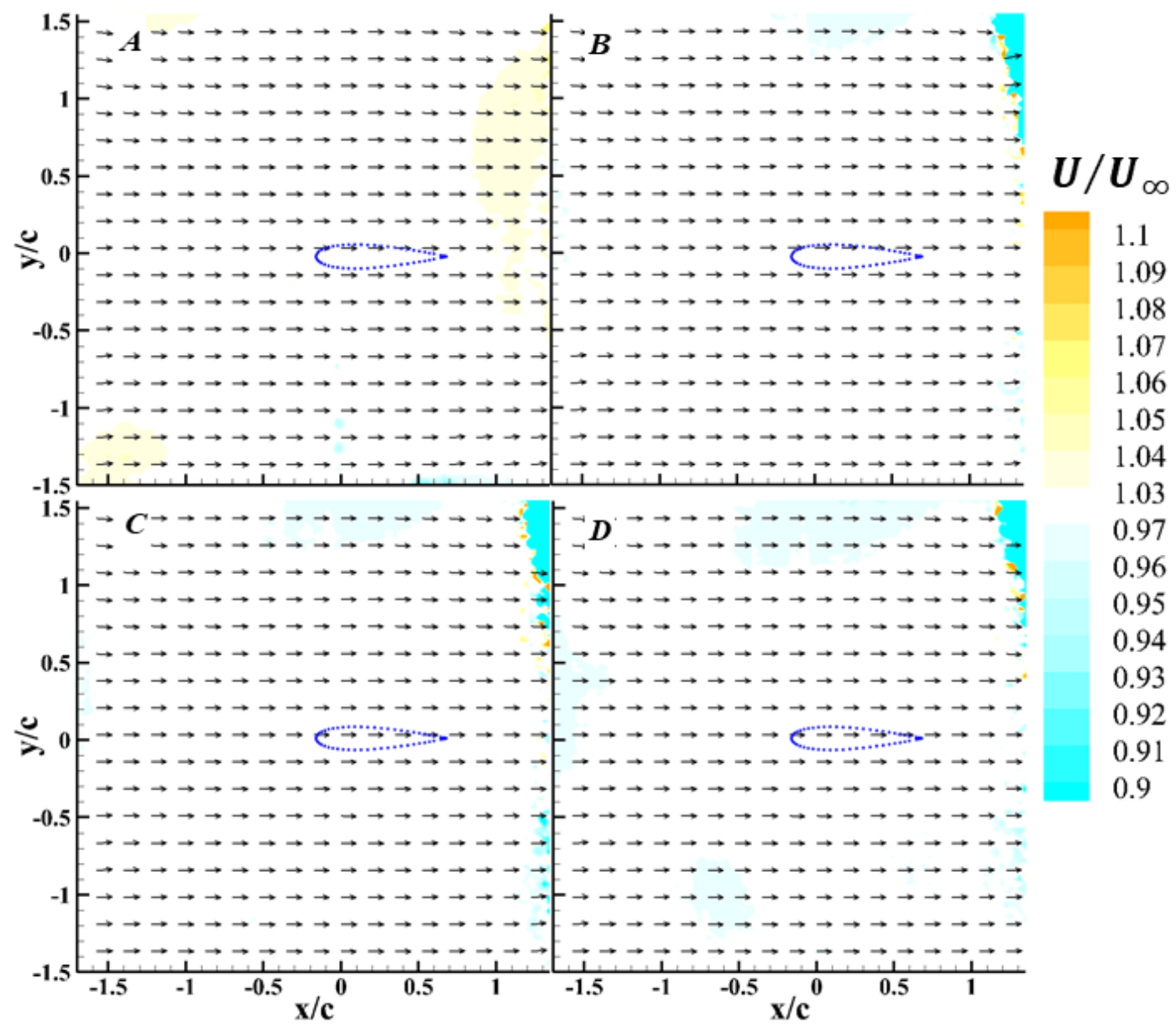

Figure 27: $U / U_{\infty}$ contour of the tunnel freestream.

A) Gust position 1. B) Gust position 2. C) Gust position 3. D) Gust position 4. 


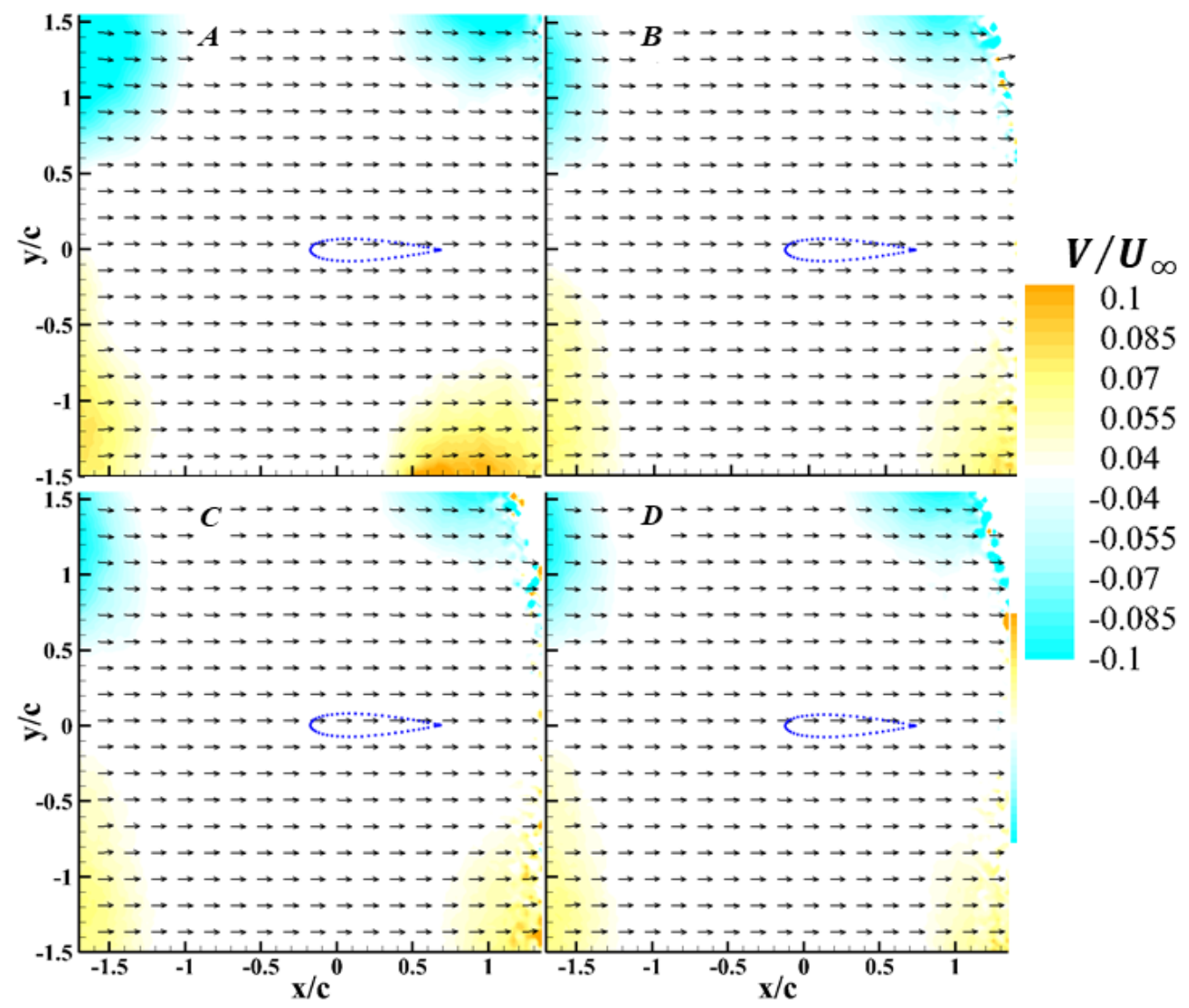

Figure 28: $V / U_{\infty}$ contour of the tunnel freestream.

A) Gust position 1. B) Gust position 2. C) Gust position 3. D) Gust position 4.

Each case above shows no change in the mean flow at the wing's location with the gust generator installed. The data shows that there was no mean vertical flow at the wing location with the gust generator installed but off, and that the mean horizontal (U) velocity of the flow was right at the desired $1.5 \mathrm{~m} / \mathrm{s}$. There were some discrepancies at the corners of the images, especially in the $\frac{V}{U_{\infty}}$ contours, but this is likely due to the large field of view and lens effects. Furthermore, there was no change seen in these images based on the position of the gust generator. This confirmed that the position the gust generator will be placed should only consider the gust's impact on the wing with no need to worry about freestream impacts. 
Figure 29 shows the U direction RMS, V direction RMS, and total RMS as a percentage of the nominal freestream flow for the gust in the farthest upstream position, Position 1. The RMS plots with the gust positioned at the other 3 positions are highly similar and therefore are left out of this report for brevity.
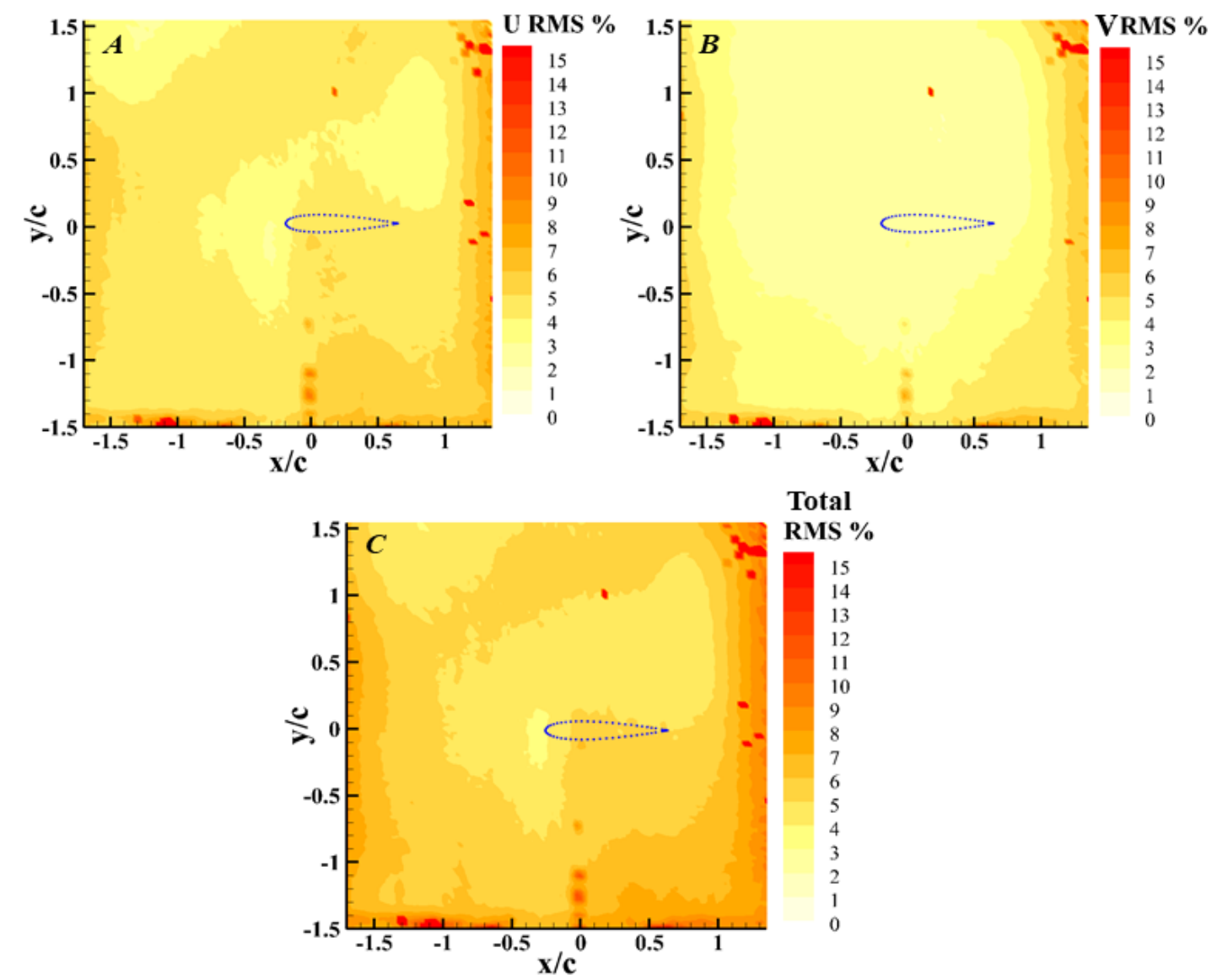

Figure 29: Tunnel freestream RMS initial characterization.

In this case the RMS values appear to be quite a bit higher than that which would be desired for experiments. It was previously concluded that the installation of the gust generator had no effect on the mean freestream, but with the velocity fluctuations shown in these RMS values, some speculation was had as to the truth in this statement, especially since it was known that the tunnel itself had been shown previously to have turbulence intensities of less than $1 \%$. However, it was hypothesized that the values of 
the $\Delta \mathrm{t}$ used for the PIV processing could have been a major factor. As specified, a $\Delta \mathrm{t}$ of $150 \mu$ s was used throughout the experiments. This was chosen as the optimal $\Delta \mathrm{t}$ value to resolve the range of flow speeds present when the gust interacted with the wing. However, with no gust or test model present the flow was much more consistent and thus a different $\Delta t$ may be required.

To accurately estimate the background RMS intensity of the flow with the gust generator installed, the value of $\Delta \mathrm{t}$ was optimized to the freestream speed and the RMS values were recalculated. Using the built in DaVIS $\Delta t$ optimization tool, an optimized value was calculated $(\Delta \mathrm{t}=499 \mu \mathrm{s})$ and the characterization was redone at Position 1. The results from these tests are shown in Figure 30. Although this freestream optimized $\Delta t$ produced better characterization results, it would not be capable of resolving later experiments with the gust active. For comparison, it was important to display the characterization completed with the $\Delta \mathrm{t}$ that will be used for future tests as it will aid in any future discussion of turbulence levels. 

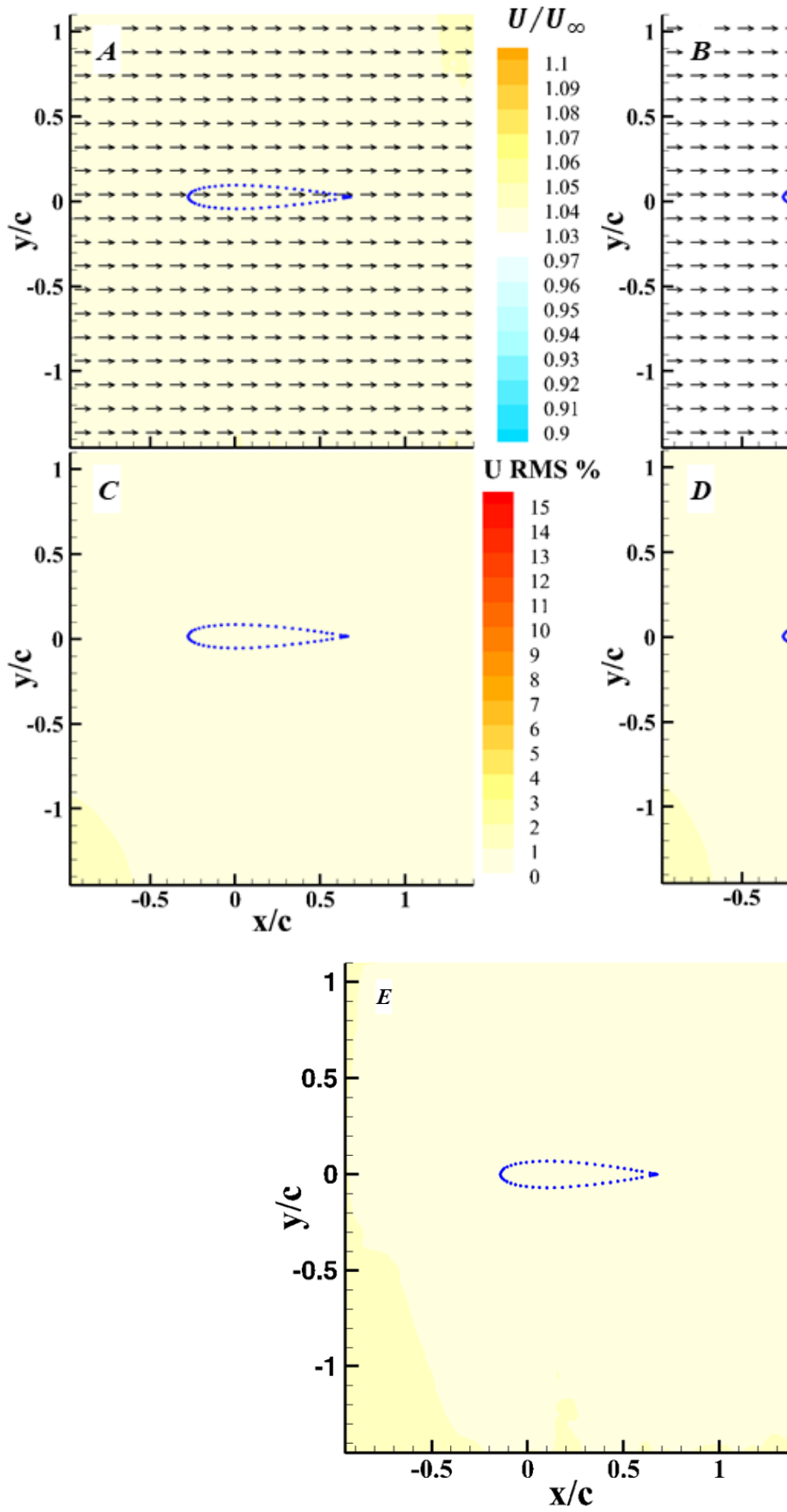
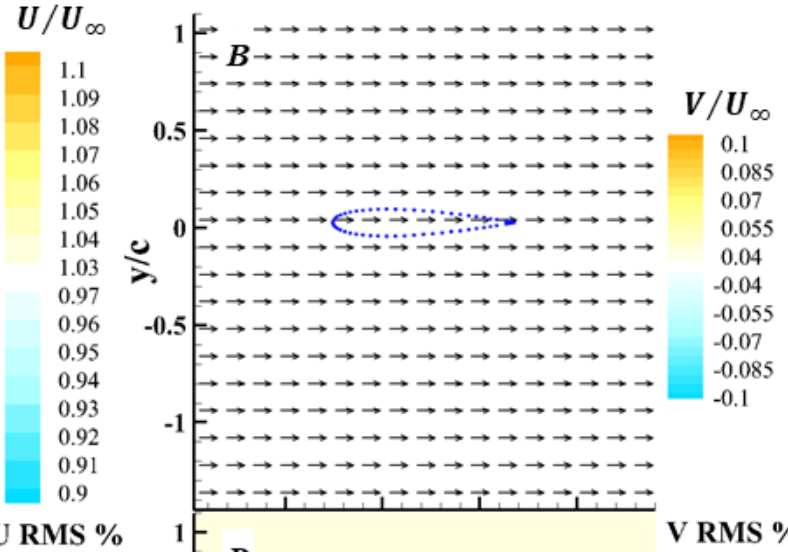

U RMS \%

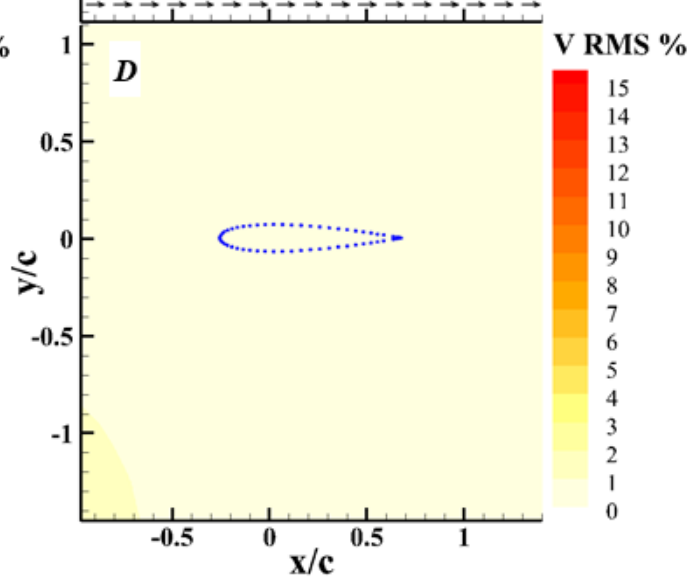

\section{Total}

RMS \%

Figure 30: Tunnel freestream characterization with updated $\Delta t$.

The RMS values using the optimized $\Delta \mathrm{t}$, as seen in images $\mathrm{C}, \mathrm{D}$, and $\mathrm{E}$ appear to have dropped to less than $2 \%$ throughout the flow. Furthermore, the $\frac{U}{U_{\infty}}$ and $\frac{V}{U_{\infty}}$ values also appear to be more uniform in these images. It was unexpected to see that the $\frac{U}{U_{\infty}}$ 
contour is not 1 throughout the flow. Instead, the contour hovered closer to a value of 1.04 , indicating a freestream flow of actually $1.56 \mathrm{~m} / \mathrm{s}$. It is extremely unlikely that this increase is an effect of the gust generator as neither the vertical (V) velocity nor the RMS values were altered. Since the tunnel speed was controlled by a fan RPM and the fan RPM to create a tunnel flow of $1.5 \mathrm{~m} / \mathrm{s}$ was previously calculated with a hot wire system, it is entirely possible that this change in horizontal flow speed is occurring due to changes in air density brought on by changes in flow temperatures between the original hot wire characterization and the current tests. Furthermore, this change of only 3 or $4 \%$ of the flow velocity was not considered a great concern while moving forward since most of the tests focus largely on the vertical components of the gusting flow.

These results lead to the final conclusion that there was no effect on the mean freestream of the flow when the generator was installed. It is important to note however that this shows one of the drawbacks of PIV data acquisition. There is obviously a $\Delta t$ value that is best at various points in a flow based on the speeds and directions of the flow at that location. However, currently the entire flow field is required to have one set $\Delta \mathrm{t}$ value when recording the PIV images. As the primary goal of this research was to examine the gust itself, this researcher picked a $\Delta \mathrm{t}$ that would show the best results inside the gust, sacrificing a small amount of accuracy in the flow outside of the gust. This point is important to remember when looking at future RMS plots in this report.

With the baseline uncertainty, measured here by the total RMS velocity, an estimate of velocity uncertainty and uncertainty in the Reynolds number can be made. While the RMS at $\Delta \mathrm{t}=150 \mu$ s was high, suggesting an uncertainty in velocity of $+/-7 \%$ of freestream near the wing, the method for collecting and calculating this RMS 
indicates that as an extreme upper bound on the uncertainty in velocity. Optimizing the value of $\Delta \mathrm{t}$ showed that uncertainty was isolated to noise within the PIV, a significant portion of which was removed using a technique discussed in section 3.5. This optimization process also provided a better estimate for the uncertainty in Reynolds number associated with the experiments. Accounting for only velocity variations of $5 \%$, and not for changes in density or viscosity, the uncertainty in Reynolds number, based on the optimized $\Delta \mathrm{t}$ PIV results, was $\operatorname{Re}=12,000+/-600$.

\subsection{Conducted Experiments}

Following the freestream characterization, the gust was characterized with both no freestream and the nominal freestream. Furthermore, tests were conducted to determine if the flow produced was actually $2 \mathrm{D}$ in nature. The results from these characterizations can be seen in Chapter 4 of this report and the full test matrix for these characterizations can be seen in Table 2 and Table 3 .

Table 2: Characterization test matrix.

\begin{tabular}{|c|c|c|c|c|c|c|c|}
\hline \multirow[b]{2}{*}{$\begin{array}{c}\text { Position } 1(13 \mathrm{~cm} \\
\text { upstream })\end{array}$} & \multirow{2}{*}{$\begin{array}{c}\text { No } \\
\text { Gust } \\
\mathrm{U}_{\infty}=1.5 \\
\mathrm{~m} / \mathrm{s}\end{array}$} & \multicolumn{2}{|c|}{ Full Power Gust } & \multicolumn{2}{|c|}{ Mid Power Gust } & \multicolumn{2}{|c|}{ Low Power Gust } \\
\hline & & $\begin{array}{c}\mathrm{U}_{\infty}=0 \\
\mathrm{~m} / \mathrm{s}\end{array}$ & $\begin{array}{c}\mathrm{U}_{\infty}=1.5 \\
\mathrm{~m} / \mathrm{s}\end{array}$ & $\begin{array}{c}\mathrm{U}_{\infty}=0 \\
\mathrm{~m} / \mathrm{s}\end{array}$ & $\begin{array}{c}\mathrm{U}_{\infty}=1.5 \\
\mathrm{~m} / \mathrm{s}\end{array}$ & $\begin{array}{c}\mathrm{U}_{\infty}=0 \\
\mathrm{~m} / \mathrm{s}\end{array}$ & $\begin{array}{c}\mathrm{U}_{\infty}=1.5 \\
\mathrm{~m} / \mathrm{s}\end{array}$ \\
\hline $\begin{array}{l}\text { Position } 2(8.5 \\
\text { cm upstream) }\end{array}$ & $\begin{array}{c}\mathrm{U}_{\infty}=1.5 \\
\mathrm{~m} / \mathrm{s}\end{array}$ & $\begin{array}{c}\mathrm{U}_{\infty}=0 \\
\mathrm{~m} / \mathrm{s}\end{array}$ & $\begin{array}{c}\mathrm{U}_{\infty}=1.5 \\
\mathrm{~m} / \mathrm{s}\end{array}$ & $\begin{array}{c}\mathrm{U}_{\infty}=0 \\
\mathrm{~m} / \mathrm{s}\end{array}$ & $\begin{array}{c}\mathrm{U}_{\infty}=1.5 \\
\mathrm{~m} / \mathrm{s}\end{array}$ & $\begin{array}{c}\mathrm{U}_{\infty}=0 \\
\mathrm{~m} / \mathrm{s}\end{array}$ & $\begin{array}{c}\mathrm{U}_{\infty}=1.5 \\
\mathrm{~m} / \mathrm{s}\end{array}$ \\
\hline $\begin{array}{c}\text { Position } 3(8.5 \\
\text { cm downstream })\end{array}$ & $\begin{array}{c}\mathrm{U}_{\infty}=1.5 \\
\mathrm{~m} / \mathrm{s}\end{array}$ & $\begin{array}{c}\mathrm{U}_{\infty}=0 \\
\mathrm{~m} / \mathrm{s}\end{array}$ & $\begin{array}{c}\mathrm{U}_{\infty}=1.5 \\
\mathrm{~m} / \mathrm{s}\end{array}$ & $\begin{array}{c}\mathrm{U}_{\infty}=0 \\
\mathrm{~m} / \mathrm{s}\end{array}$ & $\begin{array}{c}\mathrm{U}_{\infty}=1.5 \\
\mathrm{~m} / \mathrm{s}\end{array}$ & $\begin{array}{c}\mathrm{U}_{\infty}=0 \\
\mathrm{~m} / \mathrm{s}\end{array}$ & $\begin{array}{c}\mathrm{U}_{\infty}=1.5 \\
\mathrm{~m} / \mathrm{s}\end{array}$ \\
\hline $\begin{array}{c}\text { Position } 4(13 \mathrm{~cm} \\
\text { downstream })\end{array}$ & $\begin{array}{c}\mathrm{U}_{\infty}=1.5 \\
\mathrm{~m} / \mathrm{s}\end{array}$ & $\begin{array}{c}\mathrm{U}_{\infty}=0 \\
\mathrm{~m} / \mathrm{s}\end{array}$ & $\begin{array}{c}\mathrm{U}_{\infty}=1.5 \\
\mathrm{~m} / \mathrm{s}\end{array}$ & $\begin{array}{c}\mathrm{U}_{\infty}=0 \\
\mathrm{~m} / \mathrm{s}\end{array}$ & $\begin{array}{c}\mathrm{U}_{\infty}=1.5 \\
\mathrm{~m} / \mathrm{s}\end{array}$ & $\begin{array}{c}\mathrm{U}_{\infty}=0 \\
\mathrm{~m} / \mathrm{s}\end{array}$ & $\begin{array}{c}\mathrm{U}_{\infty}=1.5 \\
\mathrm{~m} / \mathrm{s}\end{array}$ \\
\hline
\end{tabular}


Table 3: 2D flow check test matrix.

\begin{tabular}{|c|c|c|c|}
\hline & Full Power Gust & Mid Power Gust & Low Power Gust \\
\hline Tunnel centerline & $U_{\infty}=1.5 \mathrm{~m} / \mathrm{s}$ & $U_{\infty}=1.5 \mathrm{~m} / \mathrm{s}$ & $U_{\infty}=1.5 \mathrm{~m} / \mathrm{s}$ \\
\hline 2" from centerline & $U_{\infty}=1.5 \mathrm{~m} / \mathrm{s}$ & $U_{\infty}=1.5 \mathrm{~m} / \mathrm{s}$ & $U_{\infty}=1.5 \mathrm{~m} / \mathrm{s}$ \\
\hline 4" from centerline & $U_{\infty}=1.5 \mathrm{~m} / \mathrm{s}$ & $U_{\infty}=1.5 \mathrm{~m} / \mathrm{s}$ & $U_{\infty}=1.5 \mathrm{~m} / \mathrm{s}$ \\
\hline 6" from centerline & $U_{\infty}=1.5 \mathrm{~m} / \mathrm{s}$ & $U_{\infty}=1.5 \mathrm{~m} / \mathrm{s}$ & $U_{\infty}=1.5 \mathrm{~m} / \mathrm{s}$ \\
\hline 8" from centerline & $U_{\infty}=1.5 \mathrm{~m} / \mathrm{s}$ & $U_{\infty}=1.5 \mathrm{~m} / \mathrm{s}$ & $U_{\infty}=1.5 \mathrm{~m} / \mathrm{s}$ \\
\hline 10" from centerline & $U_{\infty}=1.5 \mathrm{~m} / \mathrm{s}$ & $U_{\infty}=1.5 \mathrm{~m} / \mathrm{s}$ & $U_{\infty}=1.5 \mathrm{~m} / \mathrm{s}$ \\
\hline
\end{tabular}

Beyond the steady state characterization, the PIV was synchronized to the opening of the fan slats of the gust fan. Data was acquired at $28 \mathrm{~Hz}$ using a microcontroller to trigger the gust with respect to the PIV laser. Each time delay was offset by $1 / 28^{\text {th }}$ of a second from the previous, achieving the $28 \mathrm{~Hz}$ characterization with a $7 \mathrm{~Hz}$ camera. A set of 50 repeated trials were done at every timestep and the vector fields from these repeated trials were remapped onto a regular grid based on the technique used in Cohn and Koochesfahani [87]. Prior to remapping the vectors onto the regular grid, poor quality vectors were identified and removed using a Mahalanobis distance calculation. In short, the Mahalanobis distance is an operator used to calculate the distance of any point from a distribution of points. In this case, the velocity in each remapped region was used as the distribution of points and each velocity vector within that region was compared to the distribution of velocities. The key advantage of this method of identifying poor quality vectors is that the vectors are compared to their neighboring vectors and not an arbitrary value for the entire flow field. For these experiments a Mahalanobis distance of 12 was used as the cutoff and this typically resulted in the removal of less than 100 vectors in regions where over 2000 vectors are included in the distribution.

Data was recorded and processed this way for an empty tunnel at all three specified gust powers. Furthermore, a wing was placed in the tunnel and its interaction 
with the actuated gust was studied at angles of attack of 0,5 , and 10 degrees at all three powers. For later comparisons the gust was kept off and a wing was held stationary at angles between 0 and 35 degrees in 5-degree increments. Time averaged PIV of the flow field was again recorded around the wing at each angle. For these tests, 500 paired PIV image pairs were recorded. Finally, a wing was pitched at a speed and magnitude matching the effective angle it experienced during actuation of the gust. These tests were recorded at $35 \mathrm{~Hz}$ with 50 time-averaged image pairs per step. These results can be seen in Chapter 5 and the test matrix pertaining to them can be seen in Table 4 .

Table 4: Gust wing interaction and actuated gust cases.

\begin{tabular}{|c|c|c|c|c|c|}
\hline \multirow{9}{*}{$\begin{array}{l}\text { Wing } \\
\text { Position }\end{array}$} & $\begin{array}{c}\text { Full Power } \\
\text { Gust }\end{array}$ & $\begin{array}{l}\text { Mid Power } \\
\text { Gust }\end{array}$ & $\begin{array}{c}\text { Low Power } \\
\text { Gust }\end{array}$ & $\begin{array}{c}\text { No Gust, } \\
\text { Stationary } \\
\text { Wing } \\
\end{array}$ & $\begin{array}{c}\text { No Gust, } \\
\text { Pitching } \\
\text { Wing } \\
\end{array}$ \\
\hline & No Wing & No Wing & No Wing & 0 degrees & \multirow{2}{*}{$\begin{array}{c}0 \text { to } 14 \\
\text { degrees }\end{array}$} \\
\hline & 0 degrees & 0 degrees & 0 degrees & 5 Degrees & \\
\hline & 5 Degrees & 5 Degrees & 5 Degrees & 10 Degrees & \multirow{2}{*}{$\begin{array}{l}5 \text { to } 19 \\
\text { degrees }\end{array}$} \\
\hline & 10 Degrees & 10 Degrees & 10 Degrees & 15 degrees & \\
\hline & & & & 20 Degrees & \multirow{2}{*}{$\begin{array}{l}10 \text { to } 24 \\
\text { degrees }\end{array}$} \\
\hline & & & & 25 Degrees & \\
\hline & & & & 30 Degrees & $\begin{array}{l}0 \text { to } 10 \\
\text { degrees }\end{array}$ \\
\hline & & & & 35 Degrees & \\
\hline
\end{tabular}

\subsection{Summary}

Figure 31 gives one final look at the experimental apparatus designed for this research and described in this chapter. The wind tunnel used for these experiments was described. The gust generator created and the design choices that went into the selection were explained in full. The installation of the generator was explained, and the wind tunnel was characterized to show that there was no negative impact on the tunnel's 
freestream flow with the generator installed. Furthermore, two $\Delta \mathrm{t}$ values were explored to show that there would be various optimal values depending on the flow conditions being explored. Finally, the remaining tests for this report were described and test matrices laid out.

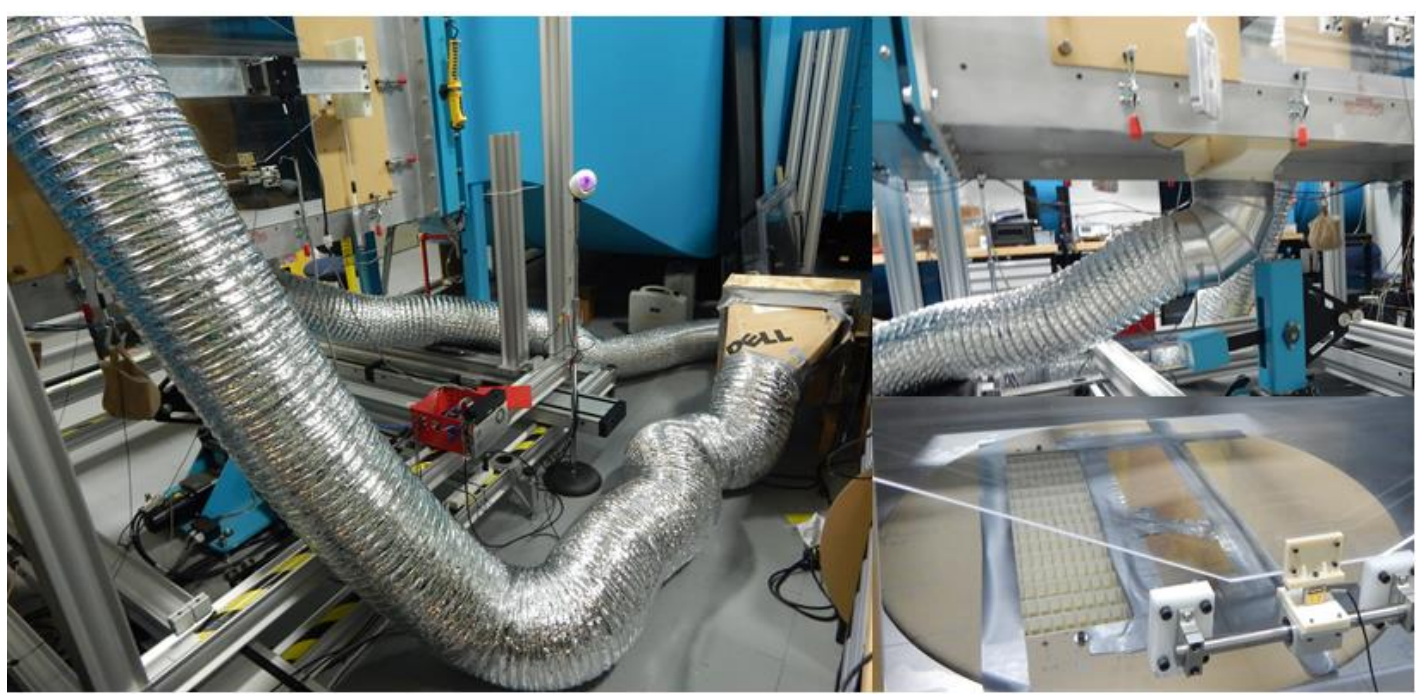

Figure 31: Final experimental setup. 


\section{Chapter 4: Gust Characterization}

The flow field created by the gust generator was characterized using 1500 PIV image pairs collected on the centerline plane of the wind tunnel, shown in Figure 32. This flow field was characterized with the gust inlet at the four locations outlined in the experimental setup section to determine the best positioning for maximum gust magnitude while limiting negative flow characteristics, such as turbulence, at the wing location. Characterization data was taken for the free gust, that is a gust without any freestream flow, and for the gust and freestream flow running at the same time. Furthermore, three separate gust powers were tested for each case and location. Once the initial characterization was completed, the flow was examined to determine the spanwise behavior and any deviation from the goal of creating a 2D gust behavior. Noting that the entire experiment is symmetrical about the center-span, characterization was done with both the tunnel and gust on at 2-inch $(0.42 \mathrm{c})$ increments from the centerline to 10 inches (2.12c) to the right of the centerline as shown in Figure 32.
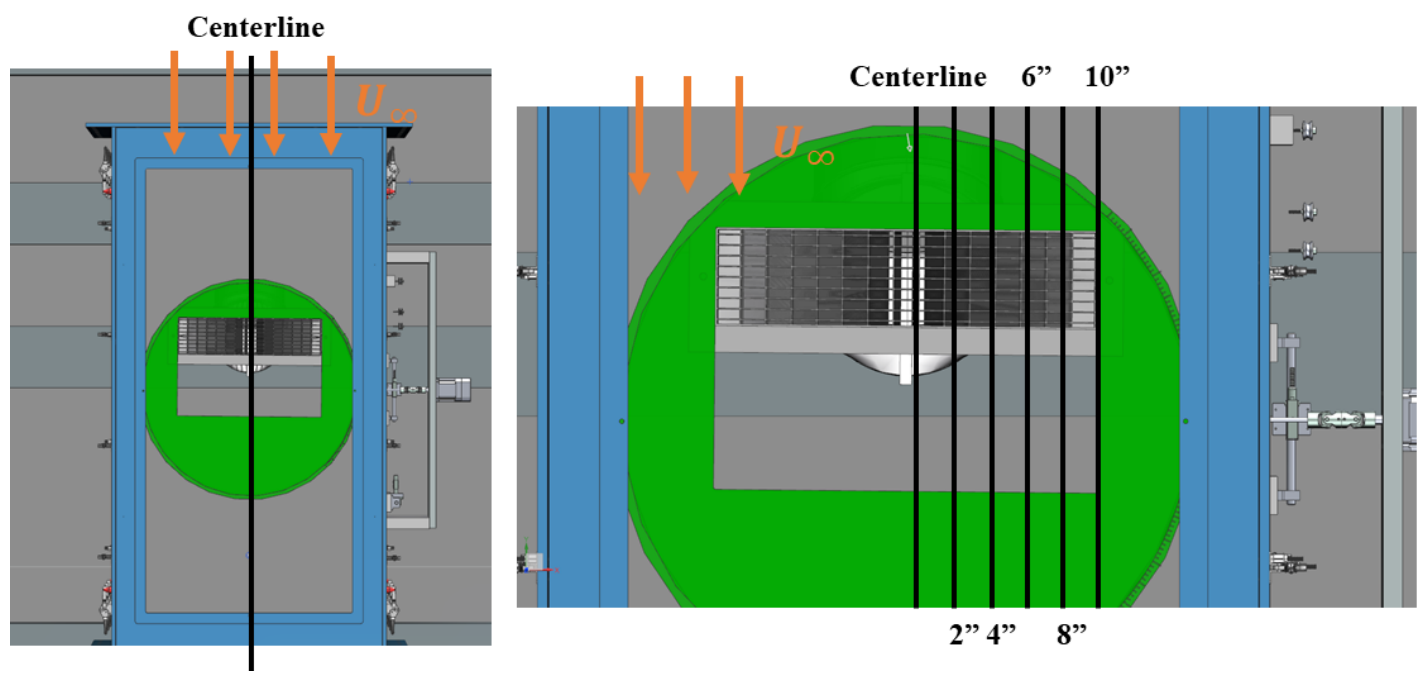

Figure 32: PIV laser locations on the wing, looking from above. 


\subsection{Gust On, Tunnel Off Characterization}

\subsubsection{Full Power Case}

After the initial characterization of the tunnel on and gust installed but turned off shown in Chapter 3, data was taken to examine the gust's static profile with the tunnel turned off. The results for the full power case can be seen in Figure 33, with contours showing vertical (V) velocity. Note: the wings shown in these images, and in all images in this section, are the locations the wing would be in these field of views. No wing was actually present in these tests.

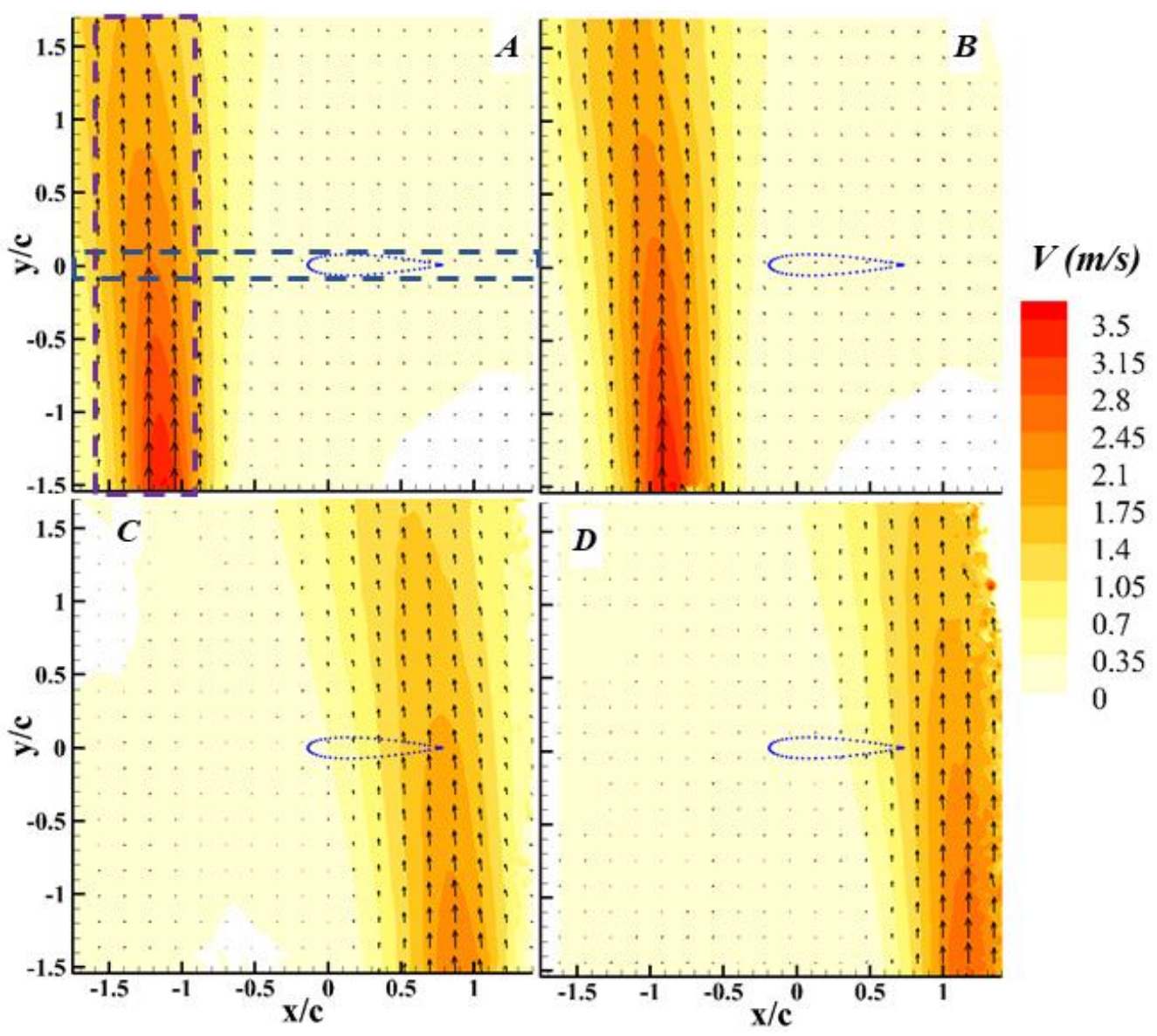

Figure 33: V velocity contours of the full power gust, no freestream. A) Gust position 1, B) Gust position 2, C) Gust position 3, D) Gust position 4. 
A few things become apparent when these images are examined closely. First, the gust at Positions 1 and 2 looks similar in shape and strength with only a change in the gust location in the field of view. The same can be said about Positions 3 and 4 . The goal was that all 4 images would be the same with only this change in location in the field of view. However, due to space restriction of the facility, in order to create the final two gust positions, an extra 180-degree turn had to be added to the flexible ducting. This turn was approximately $80 \%$ of the way through the flexible ducting path, and therefore fairly close to the gust nozzle. This turn so close to the entrance clearly had a negative impact on the gust's overall velocity and can be seen throughout the characterization plots in this section and the next.

The gust generator is shown to have produced a strong vertical velocity in the tunnel reaching about $3.5 \mathrm{~m} / \mathrm{s}$ at the bottom of the field of view and $2.5 \mathrm{~m} / \mathrm{s}$ at the planned wing location for Positions 1 and 2. Positions 3 and 4 have a maximum flow velocity of about $2 \mathrm{~m} / \mathrm{s}$ present at the bottom of the field of view and $1.5 \mathrm{~m} / \mathrm{s}$ at the planned wing location. The exact gust shape, width, and strength of the gust was determined by plotting the data extracted along the midpoint of the field of view (i.e. where the wing would be placed), shown by the blue dashed box in Figure 33 (A). The gust profiles at the marked height for all 4 positions are shown in Figure 34. 


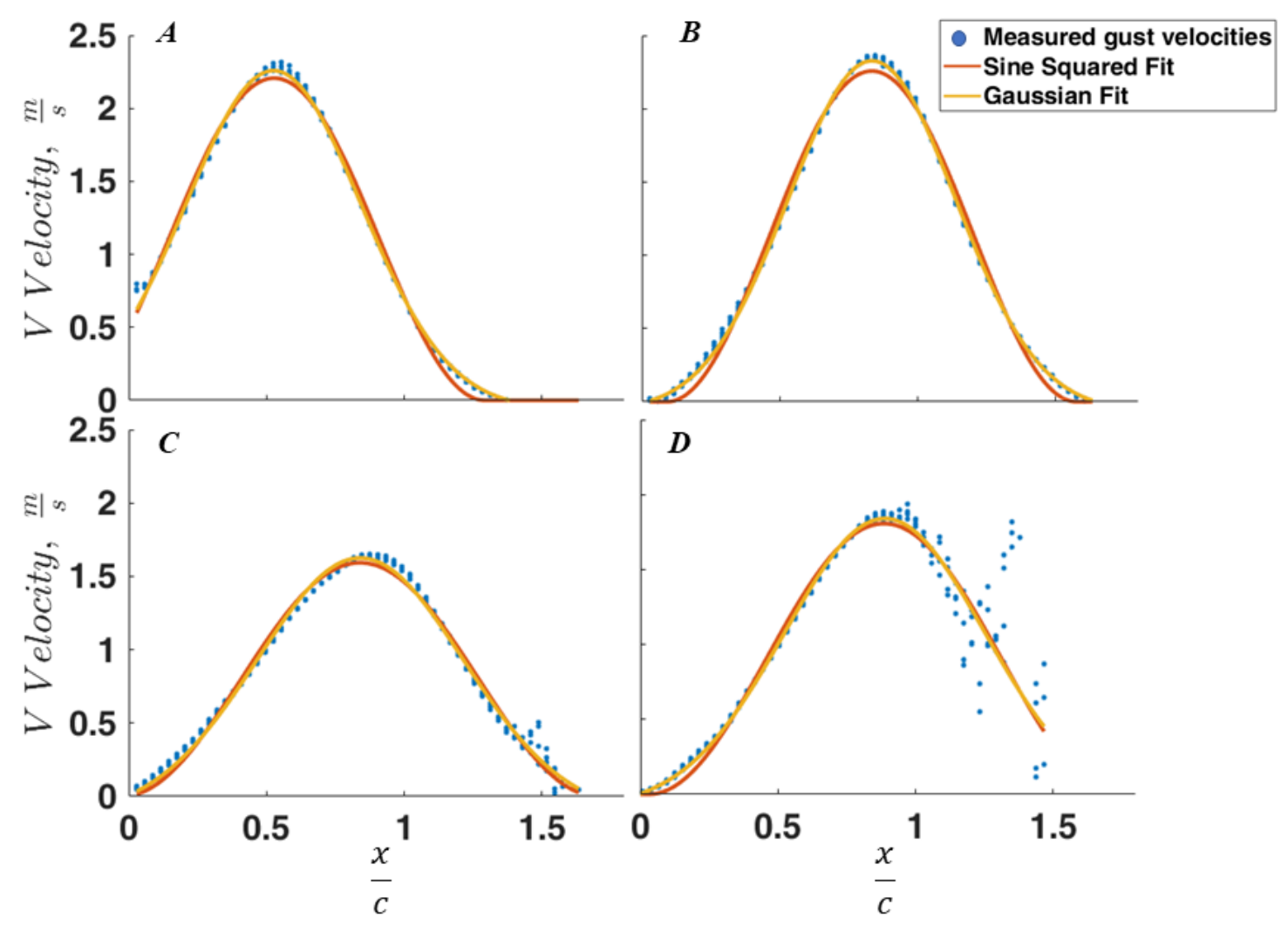

Figure 34: Full power gust profile at wing height.

A) Gust position 1, B) Gust position 2, C) Gust position 3, D) Gust position 4.

The blue dots in Figure 34 are the extracted velocities at a given $x / c$ location for the flow within the blue dashed box, Figure 33 (A). By extracting several points at a single $x / c$ location, as was done through the use of an extraction box, it was thought the trend would be more easily seen throughout these figures and later data sets presented. Furthermore, it would make any data spread, such as that seen in Figure 33 (D) more visible and warrant the appropriate attention. Note that data was extracted for the entire dashed box, but the plots were trimmed, and $x / c$ was repositioned to show just the gust profile. Both a best fit of a sine squared and Gaussian profile are plotted over the collected data points. The sine squared profile was shown to be a best fit for a similar gust experiment done in a water tow tank [73] [75]. However, for this case, the Gaussian profile is the better match. Figure 34 shows that for the further upstream 
positions (Position 1 and 2) the wing would see a vertical velocity of $2.45 \mathrm{~m} / \mathrm{s}$, while at Position 3 and 4 the velocity would be $1.70 \mathrm{~m} / \mathrm{s}$. In all four cases, the gust width was roughly 1.5 chords wide at the wing height. The left tail of the gust in Position 1 was not captured in the field of view. In Figure 33 (D), the data becomes rather sporadic towards the later portion of the curve. This region was known to be right on the edge of the PIV laser sheet and therefore, it is thought that some lack of lighting could be causing this issue. A similar extraction was done but in the vertical direction of the field of view shown via the purple box in Figure 33 (A). This was done to show how the gust slowed down as it entered the tunnel and traveled upward. These results can be seen in Figure 35.

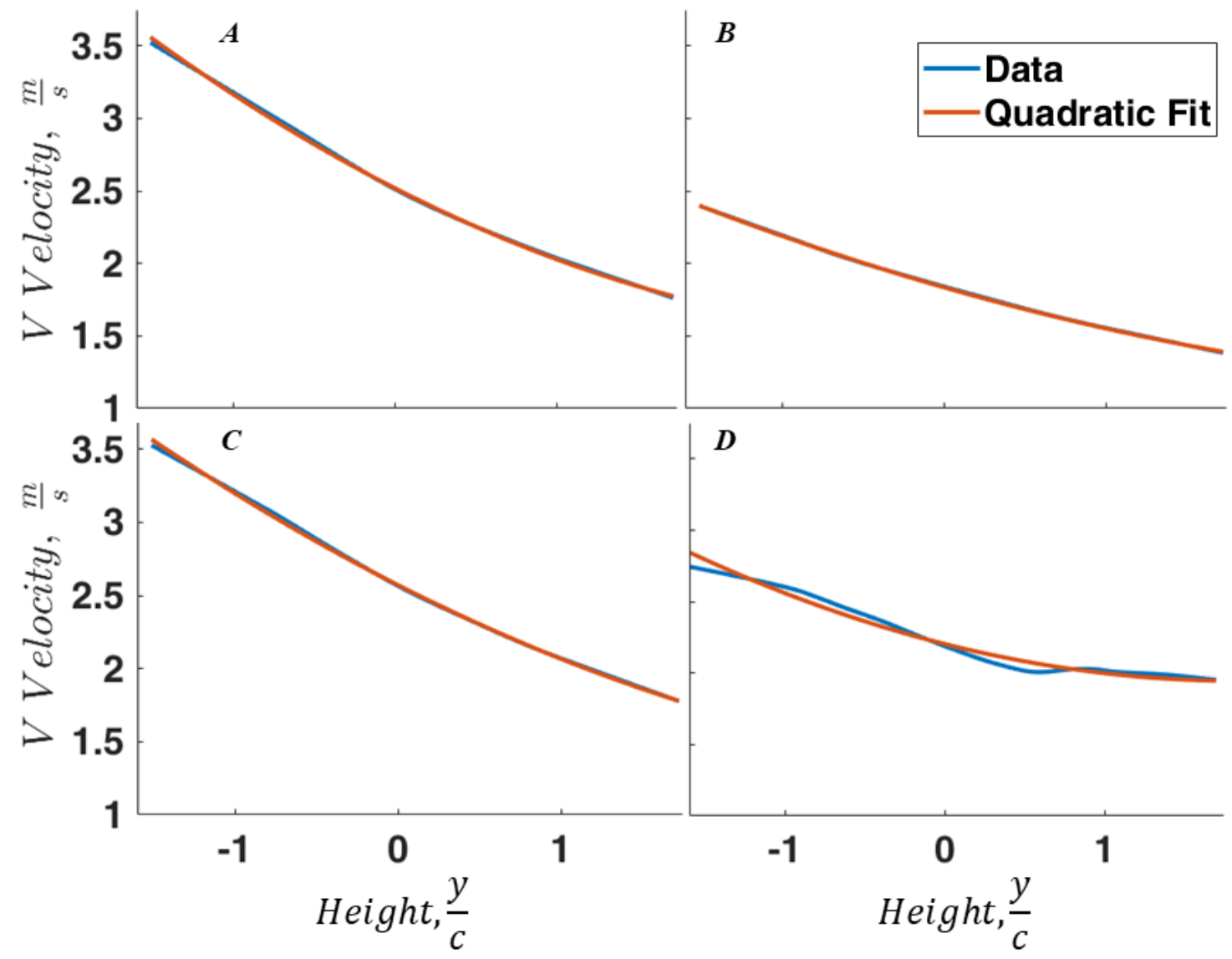

Figure 35: Vertical gust velocity vs. height in tunnel. Full power case. A) Gust position 1, B) Gust position 2, C) Gust position 3, D) Gust position 4. 
In Figure 35, the maximum gust velocity at each height in the tunnel was found and plotted creating the blue line. A best fit quadratic curve is overlaid in orange. Since the gust in Position 4 (Figure 35 (D)) was so close to the edge of the field of view, it fluctuated a bit more than the other lines. As is expected, the vertical (V) velocity slowed down as the flow approached the top of the wind tunnel. This decrease was seen to be quadratic, but with such a small arc radius, it could be loosely estimated as linear. Using this idealized linear fit, an estimated velocity of the gust leaving the nozzle of the gust generator can be extrapolated. For Position 1 (Figure 35 (A)), the maximum velocity at $y / c=-1.5$ is $3.46 \mathrm{~m} / \mathrm{s}$ and at $y / c=1.5$ is $1.84 \mathrm{~m} / \mathrm{s}$. Therefore, the slope over this region is -0.543 . Since the tunnel has a mid-height of 3.81 chords, with a velocity of $2.45 \mathrm{~m} / \mathrm{s}$ at mid-height, the estimated flow velocity leaving the gust generator is 4.52 $\mathrm{m} / \mathrm{s}$.

During the characterization of the gust it was discovered that the gust entered the tunnel at a small angle. This angle was calculated based on the average $x / c$ location at which the maximum gust strengths were found. From these locations, a sloped average line was created, and the angle of this line was estimated to be the angle of the jet. It was found to be 4.2 degrees. To further examine the effect of this slant, the horizontal (U) velocity values for the full power case at Position 1 is shown in Figure 36. 


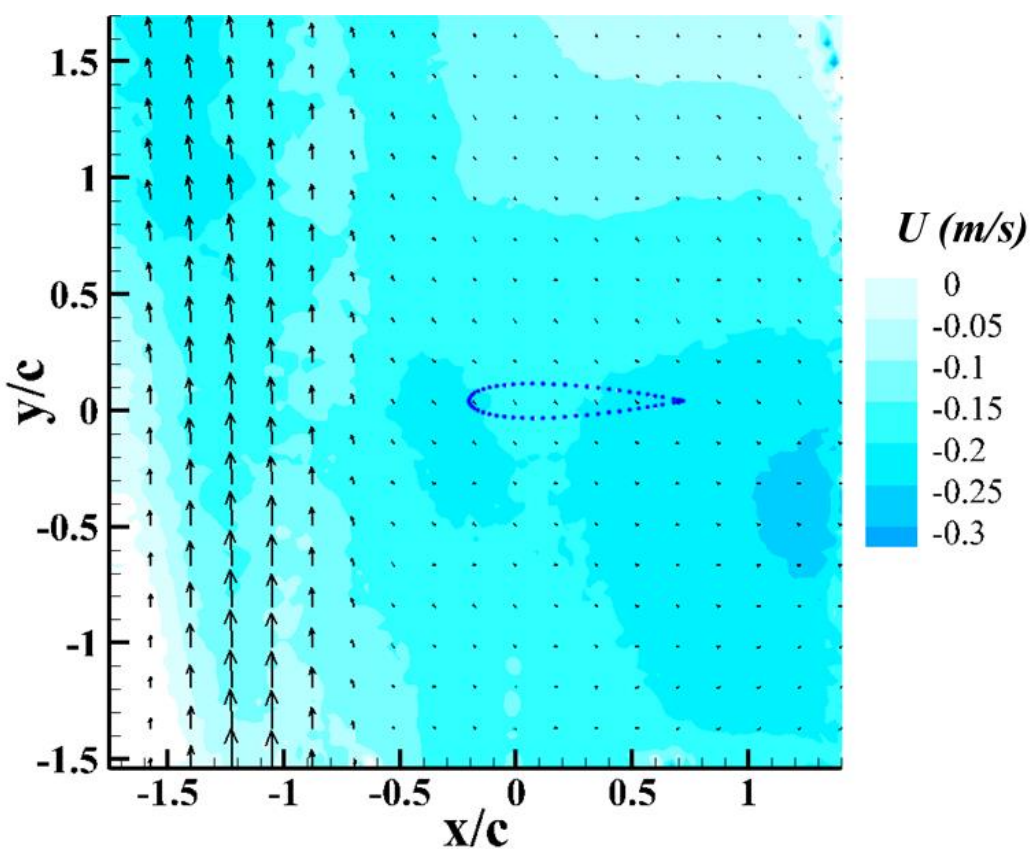

Figure 36: U velocity contour of full power vertical gust, Position 1, no freestream.

Figure 36 clearly shows some horizontal flow in the field of view. Some horizontal flow was expected, as the quiescent flow will be pulled into the gust producing some horizontal flow. Furthermore, these values found are quite low compared to the nominal freestream flow speed, just $10 \%$ for the majority of the flow. Noting that some of this horizontal velocity would be present without any gust slant, it indicates that the actual change in horizontal velocity is a bit less than $10 \%$. This, coupled with the fact that a 4.2-degree gust tilt is small in the scheme of this experiment, especially for a prototype design, meant this angle could be largely ignored for future tests and conclusions. Still, it is suggested to attach future nozzles with more than two bolt fasteners. This would remove one of the degrees of freedom from the nozzle and eliminate this slant.

The final characterization made with the gust at full power was the RMS of the flow. As shown previously, the installation of the gust generator itself had no effect on 
the RMS of the freestream flow, but it was equally as important to determine if the gust itself had any significant flow fluctuations for future calculations and force measurements. Figure 37 displays these results.
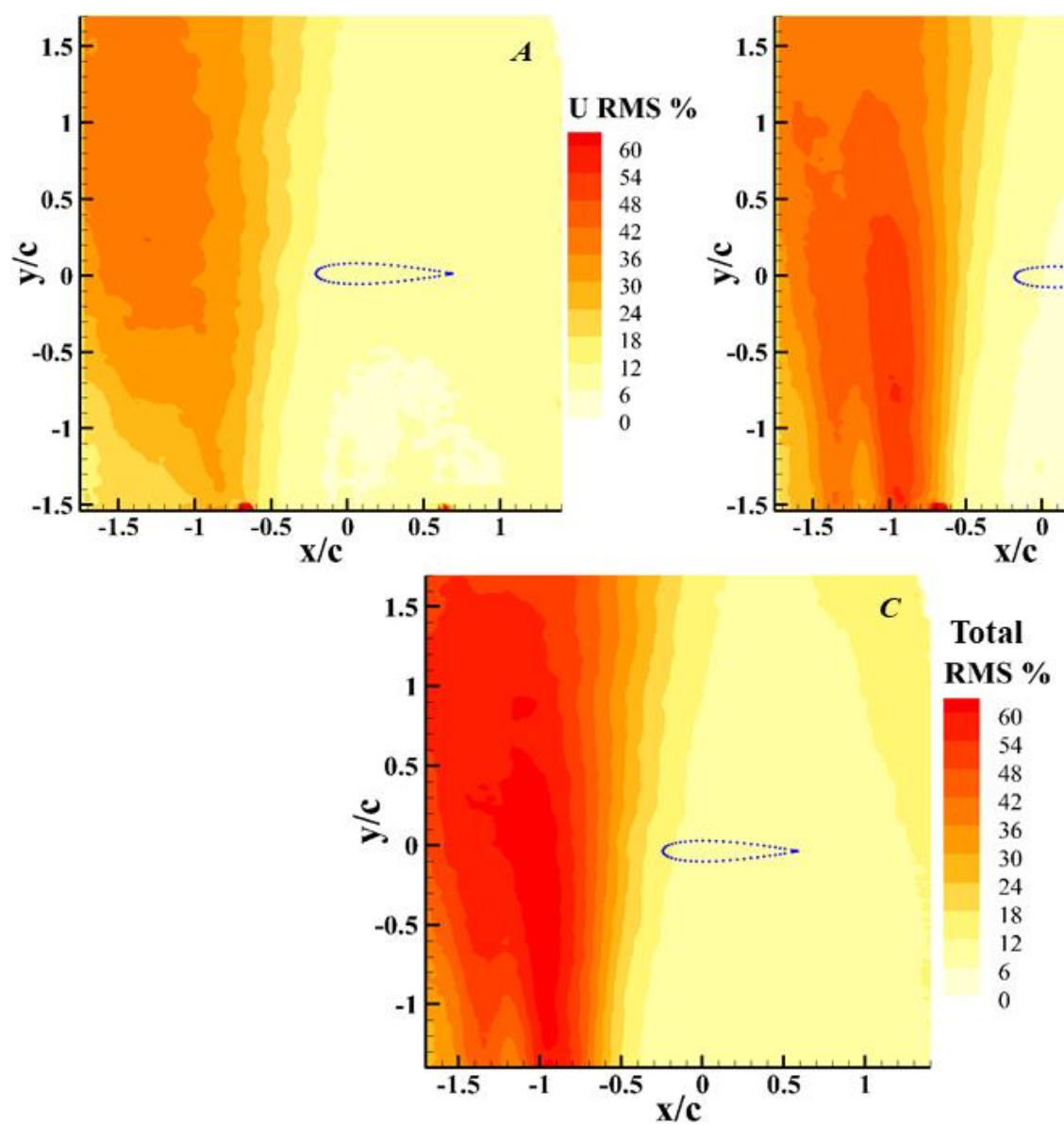

Figure 37: Full power vertical gust RMS values. Position 1.

A) $R M S$ of $U$ velocities, B) $R M S$ of $V$ velocities, C) Total flow $R M S$.

These RMS values were only calculated for Position 1 as it was assumed that the other positions would be comparable to this case. The gust itself was highly turbulent in nature. This is thought to stem from both the use of an off the shelf, non-research grade, fan as well as the use of flexible ducting in the setup that was observed to move slightly when the fan was powered. These turbulence levels were deemed acceptable based on the results of Section 4.2. A complete redesign of the system, including more 
flow straightener near the nozzle, sturdier ducting, and a different inlet would be required to further decrease the RMS values shown.

\subsubsection{Mid and Low Power Cases}

Data was also collected for the low and mid power settings. The low power setting was determined by the mid position on the rheostat turn dial, and the mid power setting was selected as halfway between the low power and full power setting, i.e. $3 / 4^{\text {th }}$ power. These settings were not expected to be linear and thus fan power is discussed as full, mid, and low-power conditions.

Figure 38 shows the results of plotting the 3 powers, all with the gust generator at Position 1, side by side. This gives a good view of how the gust changes based on rheostat setting. The low power case decreased in velocity to about half of that of the full power case, while the mid-power case seems to fall almost exactly in between, as was desired. Not much is seen in the change in gust width from these images. For the full set of results collected at these two powers and at all four positions, see Appendix

A.1.

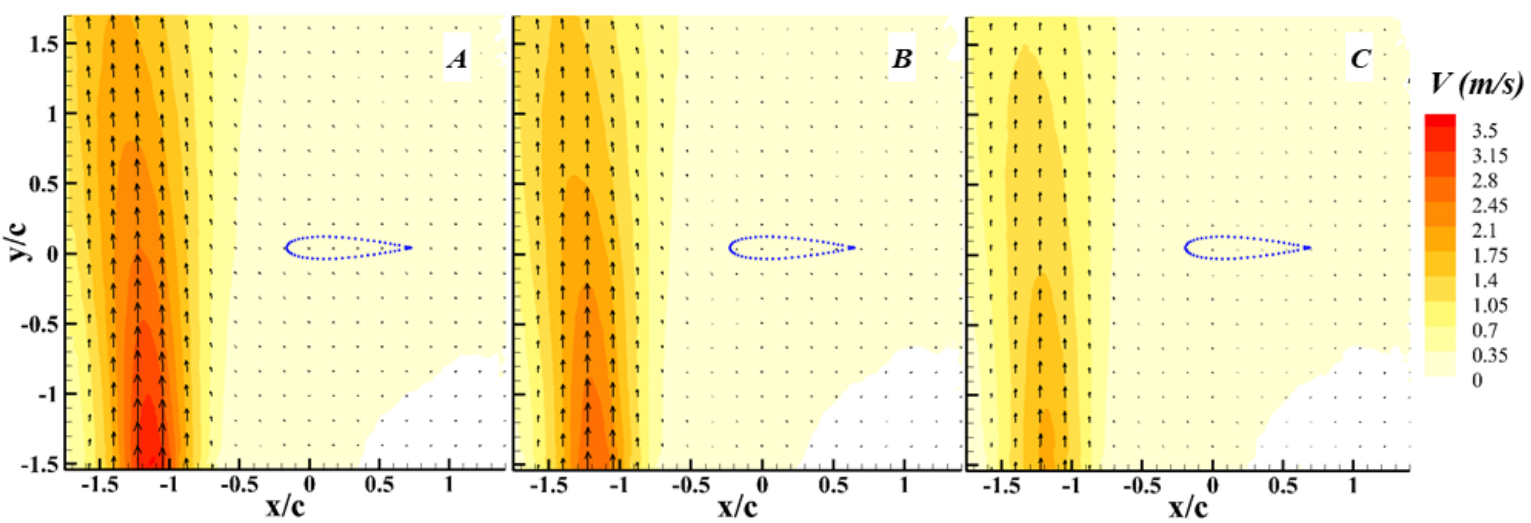

Figure 38: V contour of various vertical gust powers. Position 1.

A) Full power, B) Mid power, C) Low power. 


\subsection{Gust on, Tunnel on Characterization}

\subsubsection{Full Power Case}

A final characterization was done by having both the gust generator and tunnel on at the same time in a steady condition. As before, all 4 locations and 3 powers were characterized with a particular interest on the flow behavior at the wing location. The full power results of the collected PIV images are shown in Figure 39 with contours of normalized velocity, $\frac{U}{U_{\infty}}$.

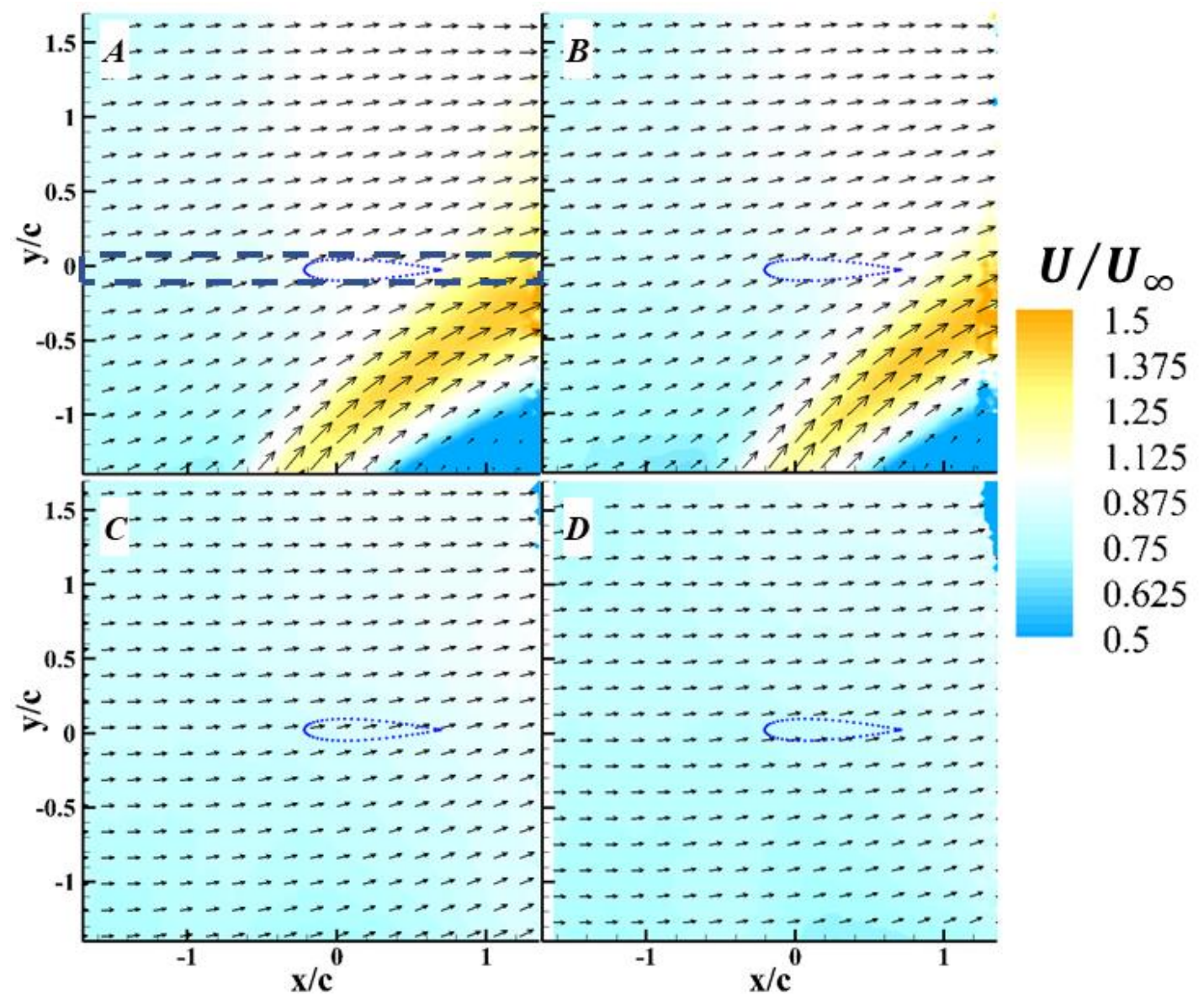

Figure 39: Steady state gust freestream interaction, full power.

A) Gust position 1, B) Gust position 2, C) Gust position 3, D) Gust position 4.

For the gust nozzle in Positions 3 and 4, the gust itself is not visible in these data sets. The gust was too far downstream at these positions and is bent out of the frame of 
view by the freestream. This is an important factor to consider when placing the gust for optimal wing interaction. From looking at these images, it is also apparent that the gust has a fairly significant impact on the $\mathrm{U}$ velocity throughout the flow. The $\mathrm{U}$ velocity increased directly in front of and inside the gust, while further upstream and just downstream, below the gust, the flow U velocity decreases. If the wing were placed in an area that saw too large a change in $\mathrm{U}$ velocity, it would result in the wing experiencing a large change in Reynolds number across it; a fact that would make any future modeling or analysis more difficult. However, this may be an important fact to expand on as gusts at these scales are explored in future works. It is possible that at these scales, the Reynolds number across a wing in a natural environment does change significantly through a gust encounter. If this fact can be proven, then designers and modelers will have to account for it in their works, further complicating the procedure used for these scales. However, for now the desire was to minimize Reynolds number change to remove a variable from the experimentation.

Still, it is known that this change in horizontal (U) velocity occurred in this case because the high velocity gust jet is bent by the freestream, thus exhausting some of the energy stored in the freestream and leading to a decrease in localized horizontal velocity. This jet being bent in such a way was a very predictable occurrence. A jet in a cross flow is a long-studied problem in fluid mechanics. It has often been shown that a smaller jet entering a larger moving body of fluid at some angle will be bent to follow that larger body's path. The exact path the smaller jet takes as it bends is very much determined by the size and strength of both bodies of fluid. There have been extensive works done that model this path and the interaction between the two bodies. Margason 
explored many of the models for the paths a gust may follow [88]. Lee and Jones described a mathematical model derived from an experimental database that helps to determine the dilution rate of the jet as it enters the cross flow [89]. As a final reference, Krothapalli et al. showed the interaction of the flow just upstream of the jet [90]. However, for these experiments the velocity ratio between the jet and the cross flow are significantly greater than that being considered in the current work. The current gust generator could be considered by some a very large jet in a crossflow problem, and therefore it may be important to some to know of the fluid interaction, and thus why these papers are presented. Still, the current study largely focused on the area outside of the jet. The actual path the flow takes was unimportant compared to the vertical component of the flow when attempting to recreate a gust. Therefore, these past efforts to study a jet crossflow are not used as a source of comparison in this report.

It was shown that the bending of the gust impacted the upstream flow by slowing it. Furthermore, this bent jet acted as an aerodynamic ramp that the freestream flow must navigate around. To determine exactly what $U$ velocity values, or change in values, the wing would see at each of the gust locations, an extraction was taking across the field of view at the wing location. The region over which the data was extracted is shown as the blue dashed box in Figure 39. The plotted points from these extractions can be seen in Figure 40. 


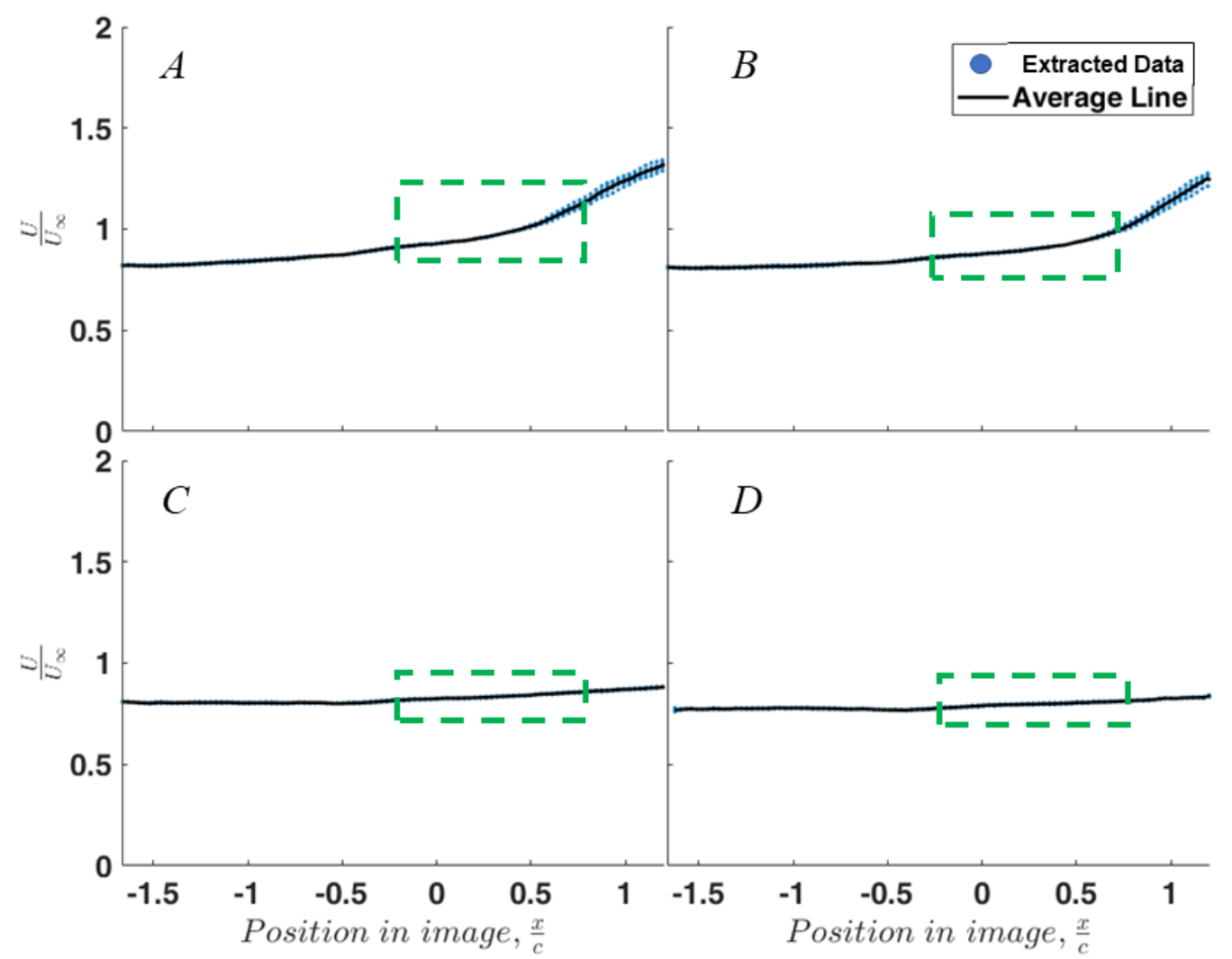

Figure 40: Extracted $U / U_{\infty}$ velocities at wing location for full power case.

A) Gust position 1, B) Gust position 2, C) Gust position 3, D) Gust position 4.

Note that the dashed green box shown in each of the images is the location of the wing's position within the flow. The data in Figure 40 shows that the gust causes the flow upstream of the ultimate wing location to slow by around $10 \%$. When looking at these $\frac{U}{U_{\infty}}$ values, there was a slight upwards trend over most of the wing location. For the wing location with the gust generator at Positions 3 and 4, this slope was fairly negligible. However, for Positions 1 and 2, near the trailing edge of the wing, there was a significant increase in the downstream velocity. This increase is not seen in Positions 3 and 4, which stay practically level across the whole wing location. Thus, ignoring any other impact from the gust, clearly Positions 3 and 4 would be the preferred locations of the wing; with one drawback being that the $\frac{U}{U_{\infty}}$ value is a bit lower than the 
desired value of 1 . When one looks at the $\frac{U}{U_{\infty}}$ value at the wing location for Positions 1 and 2, while sloped, the mean of this region does seem to be about 1 . In fact, for Position 1 , the mean value inside the dashed box was found to be 0.98 , just $2 \%$ lower than the ideal.

As stated, the position of the gust to minimize change in $U$ velocity across the wing is important to consider for final gust placement. Another obvious important selection is based on the size of the gust ratio at the wing's location. This is displayed in Figure 41.

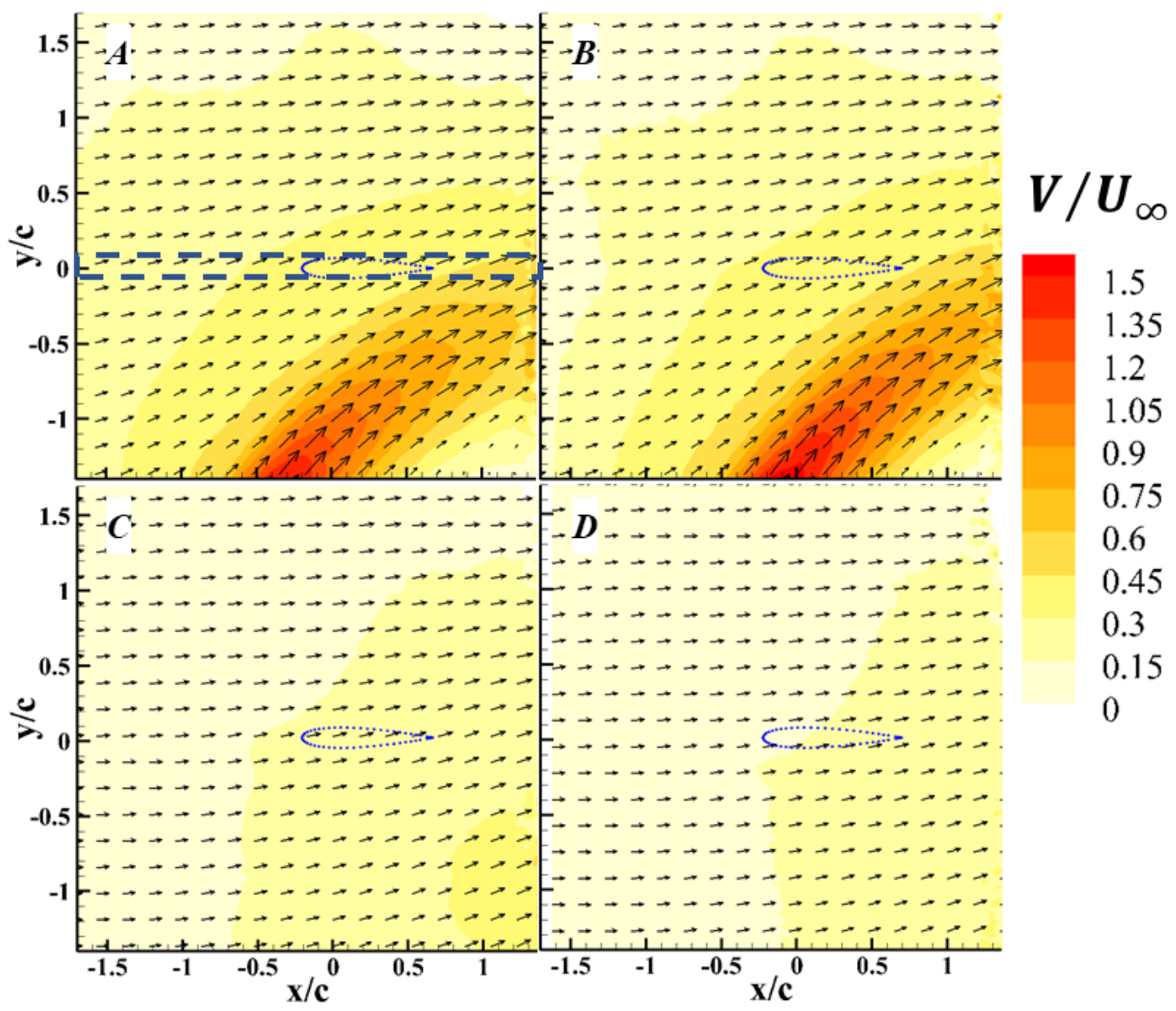

Figure 41: Gust ratio for full powered gust.

A) Gust position 1, B) Gust position 2, C) Gust position 3, D) Gust position 4. 
These steady state characterizations show that there was a significant vertical velocity at the wing's planned location. This data also shows how much effect the freestream has on the gust. In the no-freestream plots from section 4.1 the gust was clearly upstream of the wing at both Positions 1 and 2. However, with the tunnel turned on the maximum portion of the gust was downstream of the wing in both positions. Seeing as this occurred at the two further upstream positions, it is clear that Positions 3 and 4 were too far downstream to be effective. In fact, a "Position 0" further upstream still than Positions 1 and 2 would be desired. Unfortunately, as stated in the experimental setup, Position 1 was as far forward in the tunnel as the setup would allow. It was also seen that the vertical velocity in the image has decreased from about $3.5 \mathrm{~m} / \mathrm{s}$ to $2.25 \mathrm{~m} / \mathrm{s}$, a significant, but expected, decrease. To better show the gust ratio at the wing location for each gust position, data was again extracted across the frame at the wing height. The plots from this extraction are displayed in Figure 42. 


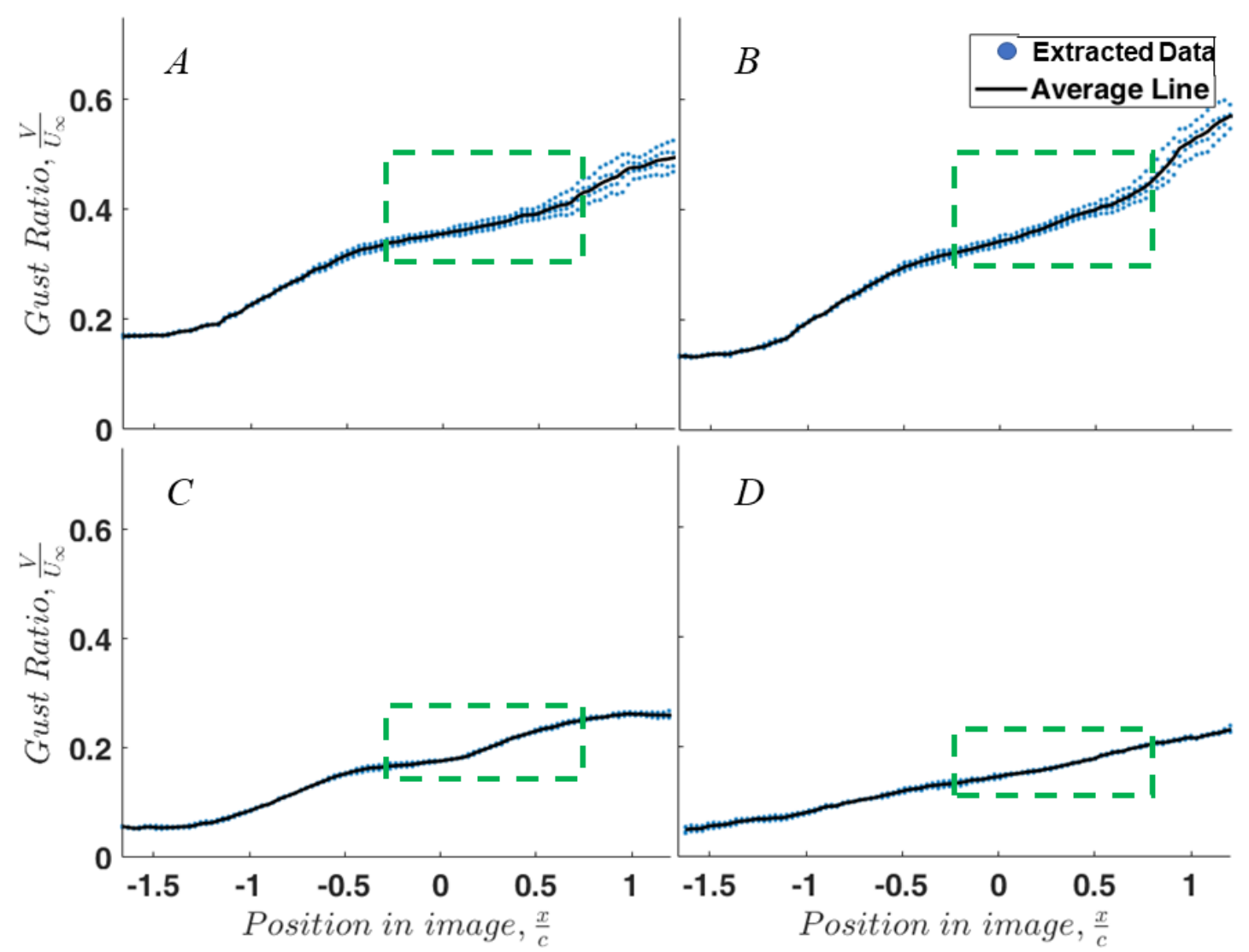

Figure 42: Gust ratio extraction at wing height for the full power case.

A) Gust position 1, B) Gust position 2, C) Gust position 3, D) Gust position 4.

Examining these figures, it is easy to see that Positions 1 and 2 had significantly higher gust ratios than Positions 3 and 4. The gust ratio $\left(\frac{V}{U_{\infty}}\right)$ at Positions 1 and 2 was nearly double that of Positions 3 and 4 . For all 4 cases, the gust ratio varied significantly across the wing position, marked again by the dashed green box. For Positions 1 and 2, the gust ratio ranges from about 0.35 to 0.4 , but the average inside the box at Position 1 was found to be 0.38 . This is a fairly high value for such a prototype, but the range garnered a bit of concern as one would not want the wing to see too large of variations across it. This variation would lead to some of the same issues seen by towing a wing through a stationary gust: the wing would experience varying forces across its body at different locations in the gust. However, it was largely thought that the angle of attack 
seen by each section of the wing would garner the greatest effect in terms of changes of force experienced by the wing. Therefore, as a final point of comparison between the four positions, plots with flow angle contours were created. This flow angle was calculated as the $\arctan \left(\frac{V}{U}\right)$ for the flow. $\mathrm{V}$ is assumed to be $\mathrm{V}_{\text {gust }}$ and $\mathrm{U}$ is assumed to be $\mathrm{U}_{\text {gust }}+\mathrm{U}_{\infty}$. This is important distinction to remember for the remainder of this work. This creates a good comparison between the changes in gust ratio and horizontal velocity in the flow as shown in Figure 43.

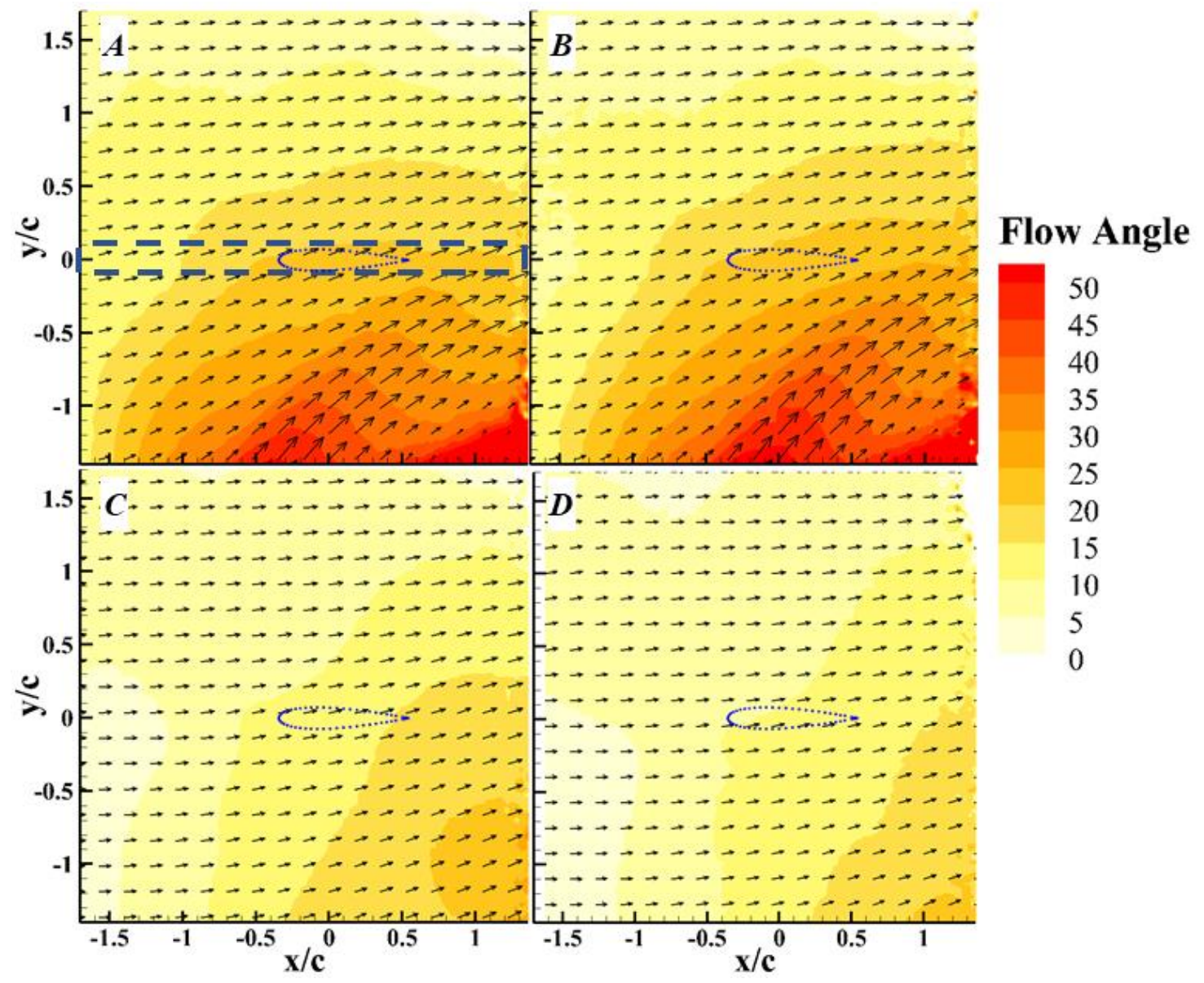

Figure 43: Flow angle for the gull power gust freestream interaction. A) Gust position 1, B) Gust position 2, C) Gust position 3, D) Gust position 4.

As was observed in the previous plots, Positions 1 and 2 produced much greater changes in angle of attack when compared to Positions 3 and 4. Positions 3 and 4 were 
too far downstream to have a large impact at the wing location. However, it is difficult to evaluate from contour plots the exact interaction at the wing location and therefore the same extraction and plots were created for flow angle. These plots can be seen in Figure 44.

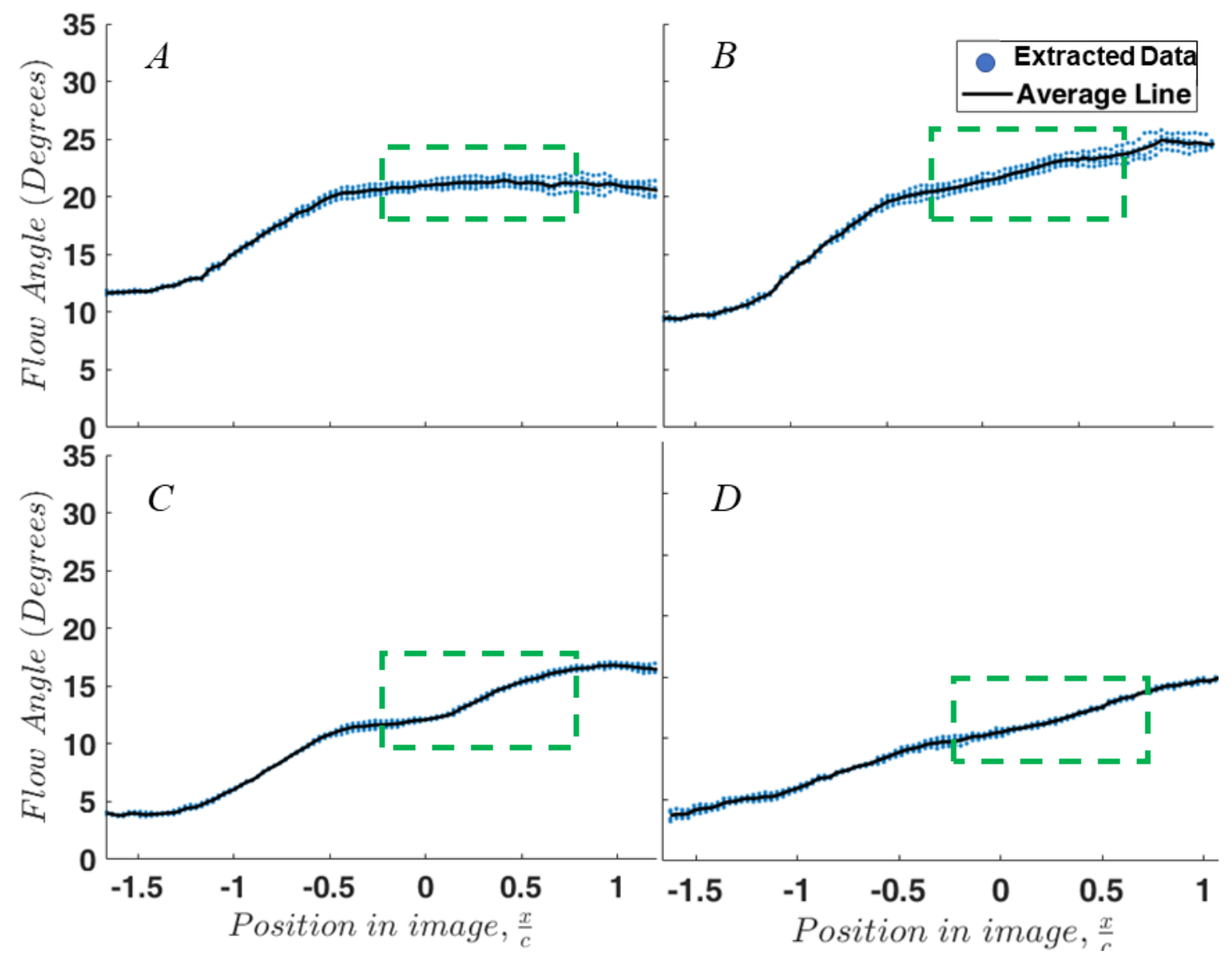

Figure 44: Flow angle data extraction at wing height for full power case. A) Gust position 1, B) Gust position 2, C) Gust position 3, D) Gust position 4.

Once the flow angle was plotted, Position 1 had a surprisingly flat region of angle of attack. The gust generator created an average change in flow angle of 21.1 degrees with the tunnel on. This is a significant change at the wing location for such a novel design prototype. Position 2 was shown to actually create an even larger change in angle of attack at some points in the flow. However, it was less flat at the wing's location. Since the goal was the maintain a constant flow angle across the wing, and 
thus hopefully gust force seen by every section of the wing, it was determined that Position 1 would be the best test location for future tests and deeper analysis. As of now, the physical reason that Positions 1 and 2 produced such different flow angle plots is unknown.

It is again important to analyze the RMS of the flow. As in the previous section, it was shown that RMS was directly proportional to the strength of the gust. Since Position 1 had a strong gust and was the planned position for future tests, only the RMS at this position was evaluated. The RMS plots can be seen in Figure 45.
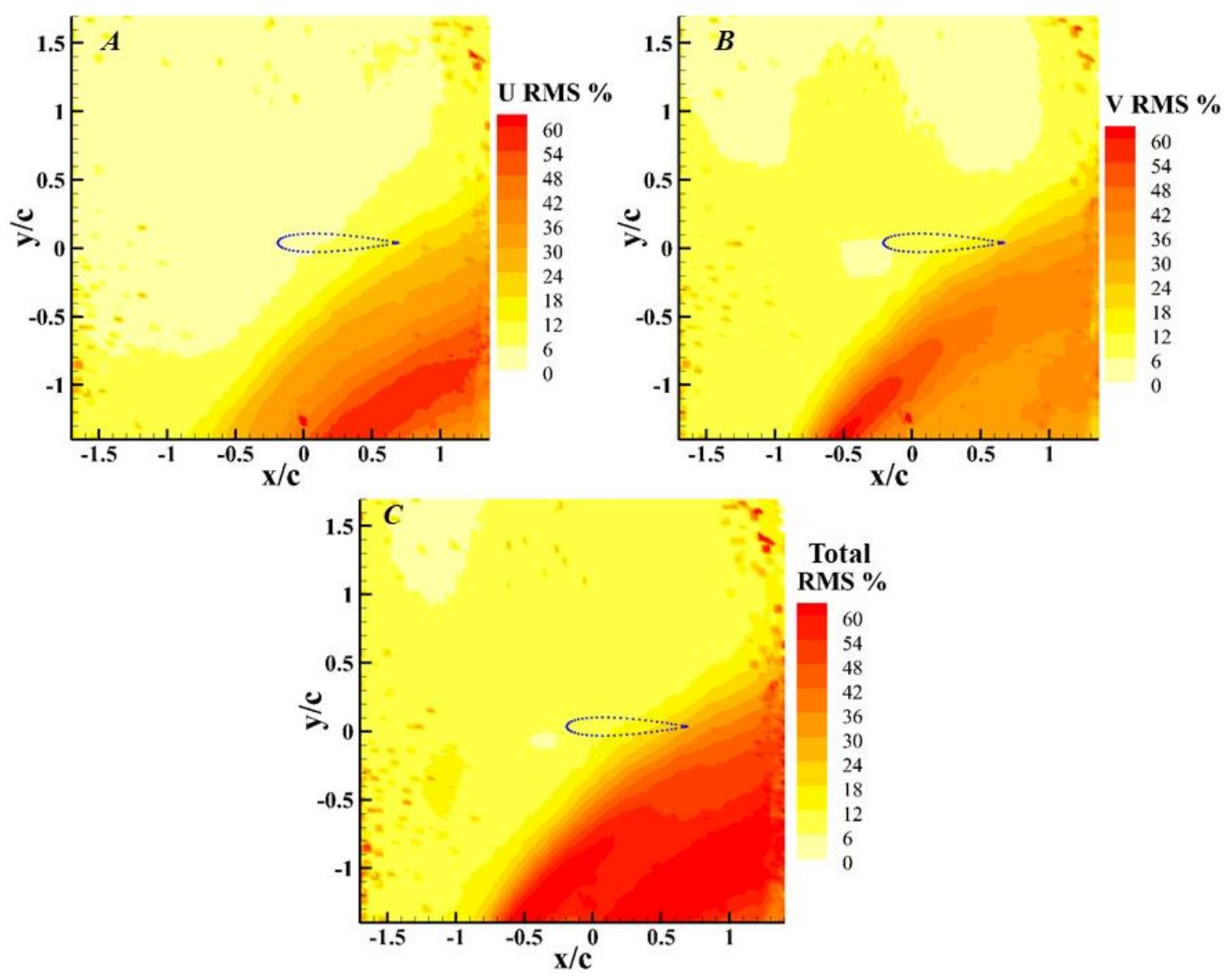

Figure 45: RMS values for the full power gust at steady state at position 1. A) U RMS percentage, B) VRMS percentage, C) Total flow RMS percentage.

The bending of the gust downstream resulted in a significantly lower than expected flow RMS at the wing location. Still, as some of the flow in the wing region 
was affected by the gust, as is desired of course, the wing location experiences some flow fluctuations. For the most part, the planned wing location experienced an RMS of less than $18 \%$. This value is still fairly high but was deemed acceptable for being the first iteration of a novel design. Furthermore, as was shown in the tunnel characterization, it is quite likely that the RMS was actually lower than that being shown here. A different $\Delta t$ value could render a lower RMS value outside of the gust where the freestream is more uniform. However, it impossible to select a single $\Delta \mathrm{t}$ value that would work best for every location in such an unsteady flow and thus the researcher chose a value that would work well, but might not be perfect, for the entire flow field. This RMS is still important to remember when analyzing future data of the gust's actuation shown in the next chapter and does explain some of the fluctuations seen.

\subsubsection{Mid and Low Power Cases}

The characterization was also completed for both the mid and low power gust cases. Since the primary focus of the remaining research will be on the gust at Position 1, only results for this case are shown in Figure 46. The remaining results and the extracted points can be found in appendix A.2. 


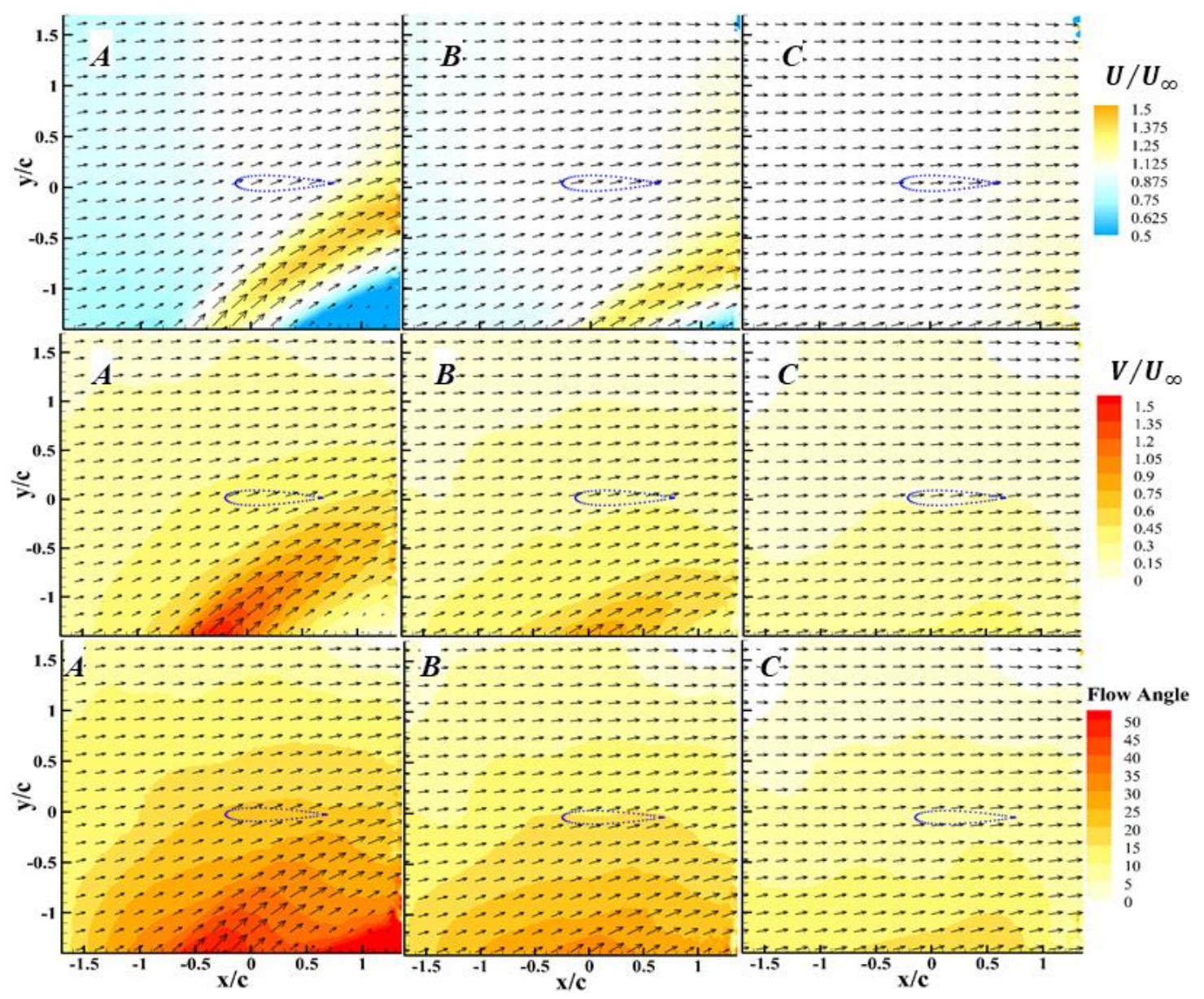

Figure 46: Full, mid, and low power comparisons for gust at Position 1.

A) Full power, B) Mid power, C) Low power.

Similar to the free-gust characterization, the main change between each power is the gust's strength. This largely manifested itself as a broadening of the bands in the flow field of constant flow characteristics, i.e. the region one could place the wing to see no change in horizontal (U) velocity, for example, increases the slower the gust jet velocity becomes. This, along with the known characteristic that the turbulence of the generator decreases with gust strength means that the lower speed settings should create results that are cleaner, albeit with smaller changes in flow angle. Furthermore, since the gust speeds are lower, the changes in horizontal (U) velocity also decreased, another important benefit to these lower speed gusts. With this fact in mind, it was determined 
that it would be best to continue to test at all three gust powers for comparison. As MAVs are likely to experience different strengths of gusts in flight, this allowed for the collection of a wider pool of data for comparison to the flight conditions they may experience.

\subsection{D Flow Examination}

One final characterization experiment was done to determine what, if any 3D effects were present in the flow field. While a full span wing was used, and the gust generator was entirely symmetric, as noted, it only spanned $55 \%$ of the tunnel and therefore 3D effects were expected. However, it was initially hypothesized that these effects would not be present well inside the $55 \%$ of the tunnel which the gust directly affected. To test this hypothesis, spanwise flow field data was taken every 2 inches $(0.42 \mathrm{c})$ from the center line to 10 inches $(2.12 \mathrm{c})$ from the centerline with the gust at all three powers at Position 1. This 10-inch from center span location corresponded to the edge of the gust inlet. Figure 47 shows the results from the full powered case in terms of $U$ velocity, which shows the percentage change in $U$-velocity when compared to the centerline. 

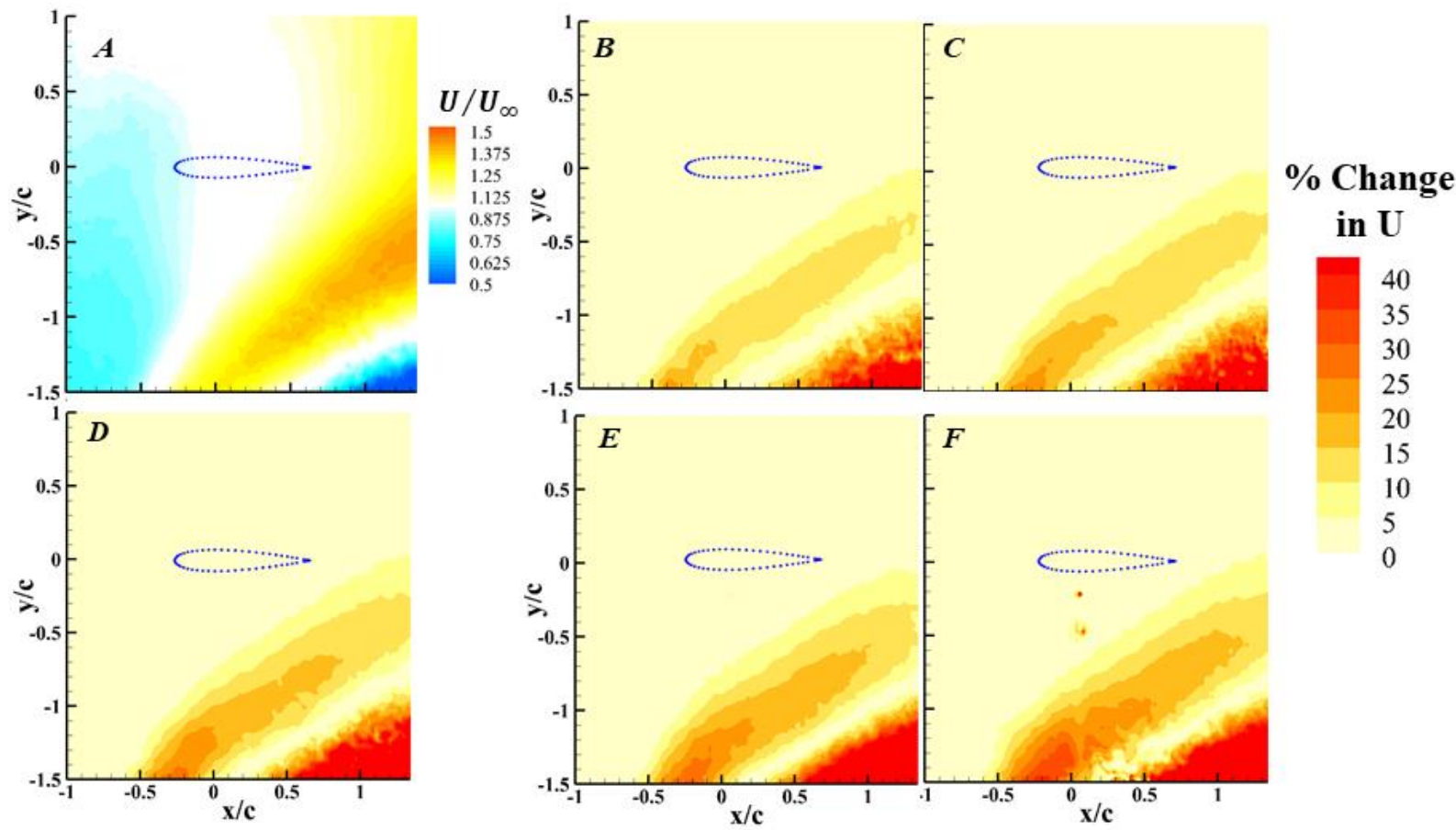

Figure 47: Change in U Velocity based on span location for full gust power.

A) Centerline U/U⿻ contour, B) 2" from centerline, C) 4" from centerline, D) 6" from centerline, E) 8" from centerline, F) 10" from centerline.

Figure $47(\mathrm{~A})$ is a contour of the $\frac{U}{U_{\infty}}$ value at the centerline of the tunnel. The field of view location was slightly different than those collected in section 4.2 as to be better centered on the gust for comparison purposes. Figure 47 (B to F) are contours of the change in horizontal flow velocity (U) when compared to that of the horizontal flow velocity $(\mathrm{U})$ at the centerline. The formula used to calculate this percentage change was:

$$
\% \text { change }=\frac{U_{\text {center }}-U_{\text {offcenter }}}{U_{\text {center }}} * 100
$$

From these plots, it was clear that inside the gust jet itself, there was a significant decrease at it spans outwards. At 2 inches from the center there was already a decrease of about $15 \%$ inside the gust. It is highly likely that this decrease in velocity is directly related to the out of plane expansion of the gust off centerline. However, at the wing 
location, there was only a change of about 3-5\%. By 10 inches from centerline, the velocity inside the gust has decreased by nearly $40 \%$ in some locations with most of the gust showing a decrease of about $25 \%$. But even this far out, at the wing location there was still only a decrease in $U$ velocity of about $5 \%$. Therefore, across this measured span, it was seen that the wing witnessed a change in U velocity of around $5 \%$ due to 3D effects of the flow. This change is deemed quite low and thus no concern for $3 \mathrm{D}$ flow effects on the $\mathrm{U}$ velocity were had.

The more impactful investigation, however, occurs when examining the change in vertical (V) velocity. This is given by the contours plotted in Figure 48.
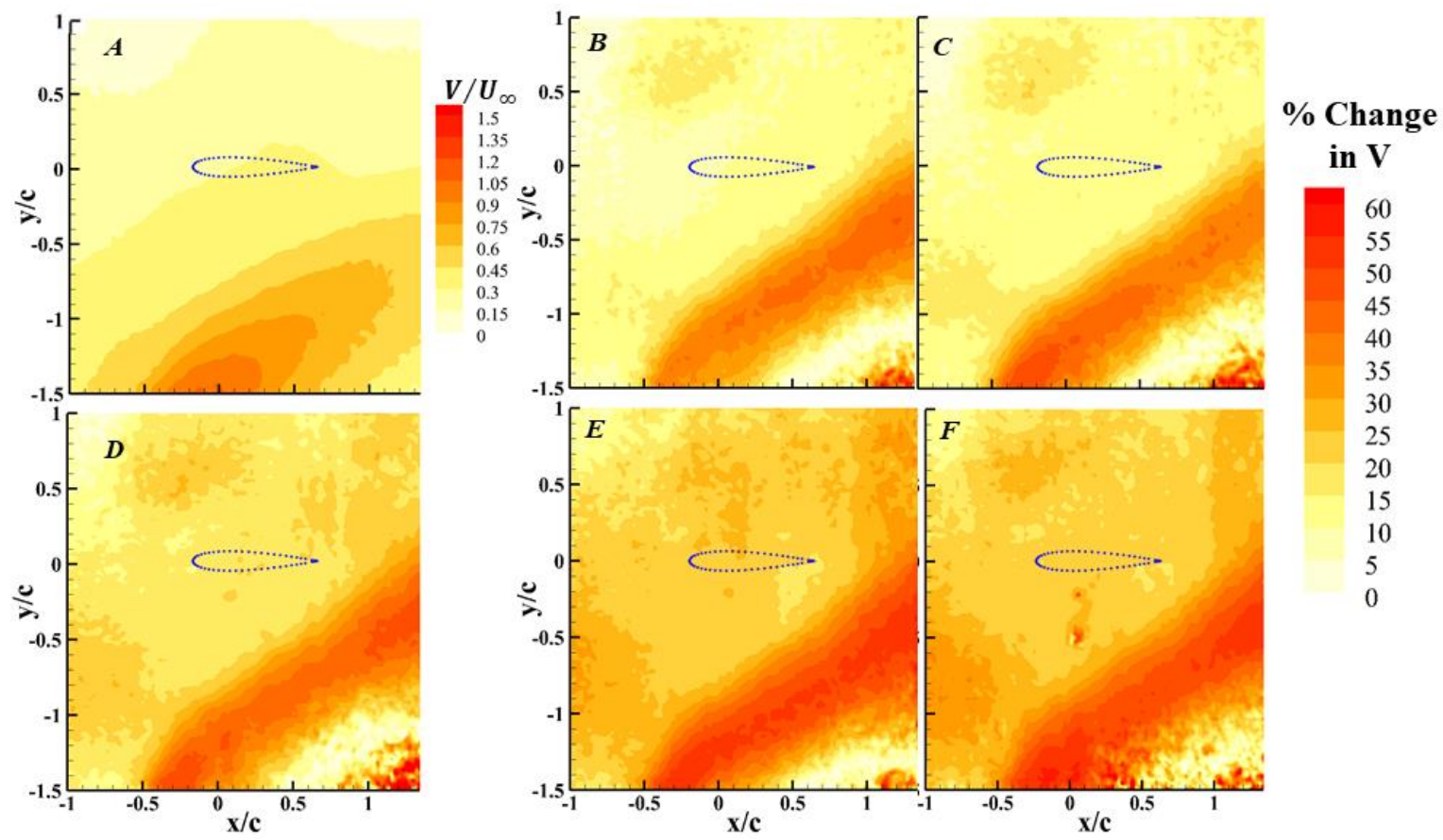

Figure 48: Change in V velocity based on span location for full power gust. A) Centerline V/U $U_{\infty}$ contour, B) 2" from centerline, C) 4" from centerline, D) 6" from centerline, E) 8" from centerline, F) 10" from centerline.

Similar to the $\mathrm{U}$ velocity, the largest change in vertical (V) velocity was inside the gust. The same change formula as before was used to calculate the change in vertical velocity throughout the flow at the various span locations but substituting the horizontal 
components with vertical ones. 2 inches from the centerline, the velocity inside the gust has decreased by nearly $40 \%$. At the wing location though, there was a smaller decrease of around 10\%. By the 10-inch mark, however, the velocity inside the gust has decreased by $60 \%$ and at the wing the velocity has decreased by $30 \%$. This is a fairly significant decrease in $\mathrm{V}$ velocity, though as stated, not unexpected due to the spreading of the gust at the edges of the generator. However, the $10 \%$ velocity decrease at the wing location just 2 inches from the centerline is of some concern and displays the greatest drawback seen so far for this system. This equates to an estimated $6 \mathrm{~cm} / \mathrm{s}$ decrease in the vertical flow speed at the wing's location. Clearly the gust nozzle is not distributing the flow as evenly had been hoped and there are 3D effects present in the flow. This is something to bear in mind moving forward with the analysis of future tests and something that will need to be addressed in future redesigns of this gust generator.

To fully characterize all cases being tested, the same spanwise measurements were carried out for both the mid and low power cases at Position 1 and are given in Figure 49 through Figure 52. 

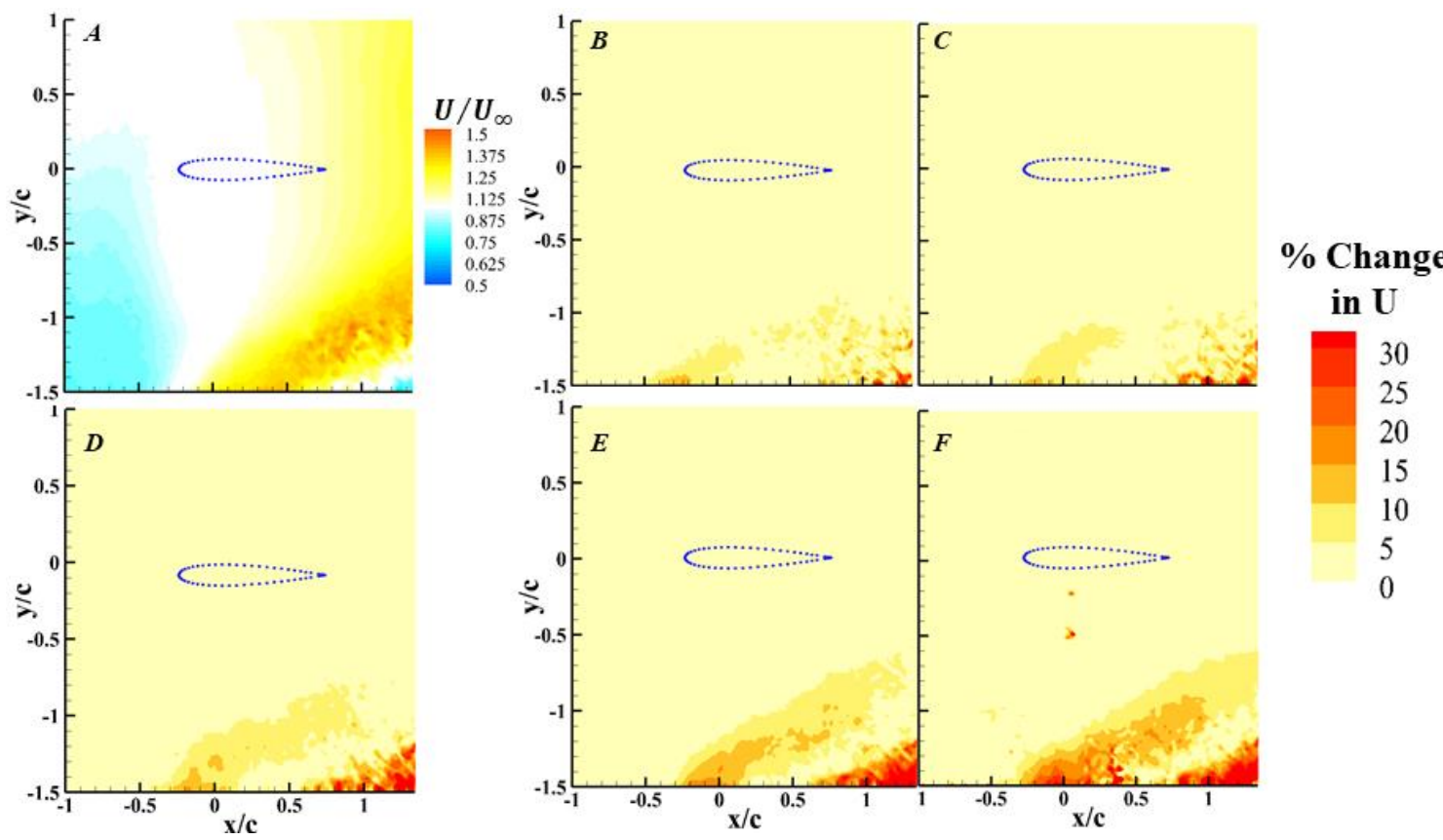

Figure 49: Change in U velocity based on span location for mid power gust.

A) Centerline $V / U_{\infty}$ contour, B) 2" from centerline, C) 4" from centerline, D) 6 " from centerline, E) 8" from centerline, F) 10" from centerline.
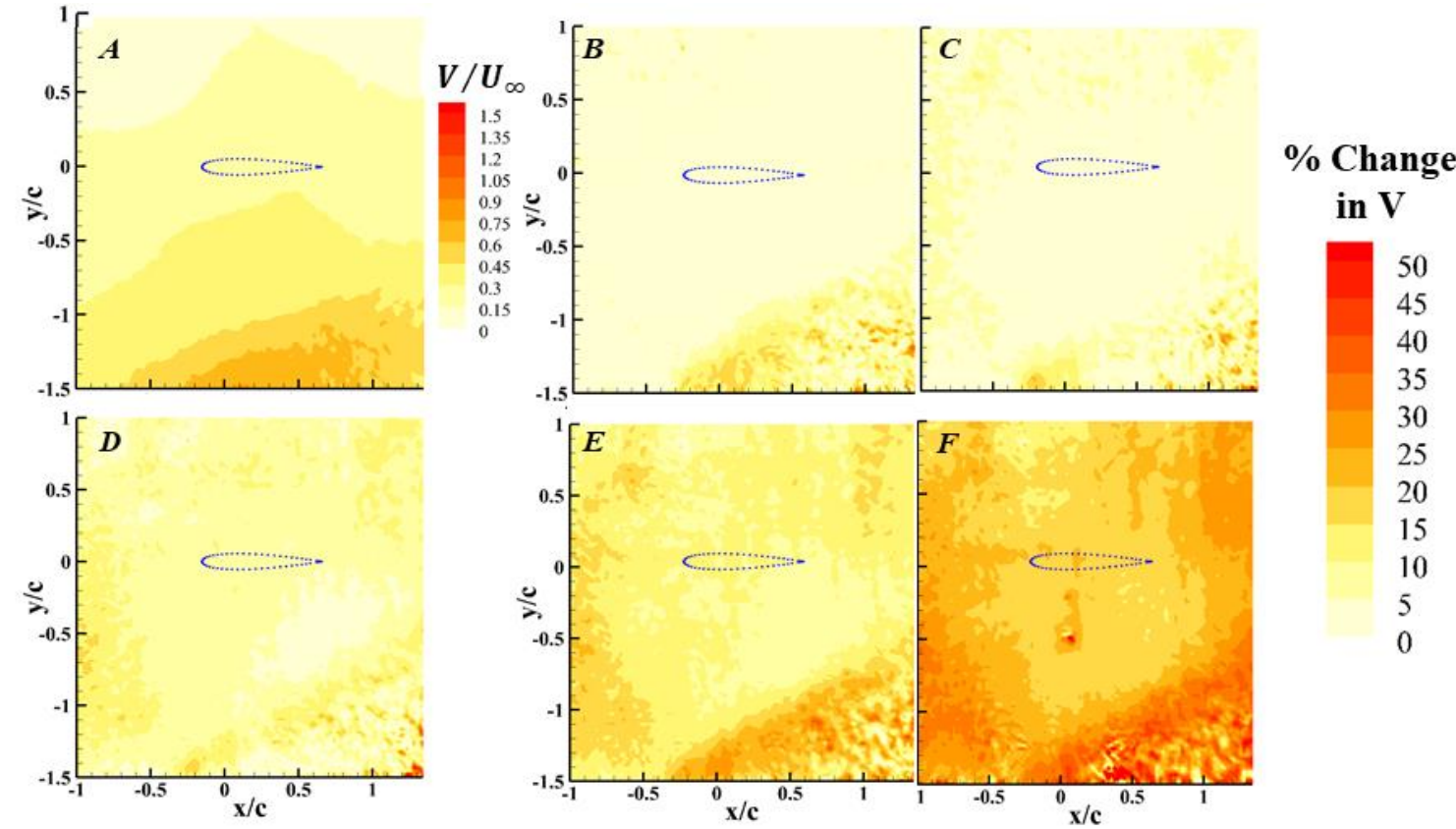

Figure 50: Change in $V$ Velocity based on span location for mid power setting.

A) Centerline $V / U_{\infty}$ contour, B) 2" from centerline, C) 4" from centerline, D) 6 " from centerline, E) 8" from centerline, F) 10" from centerline. 

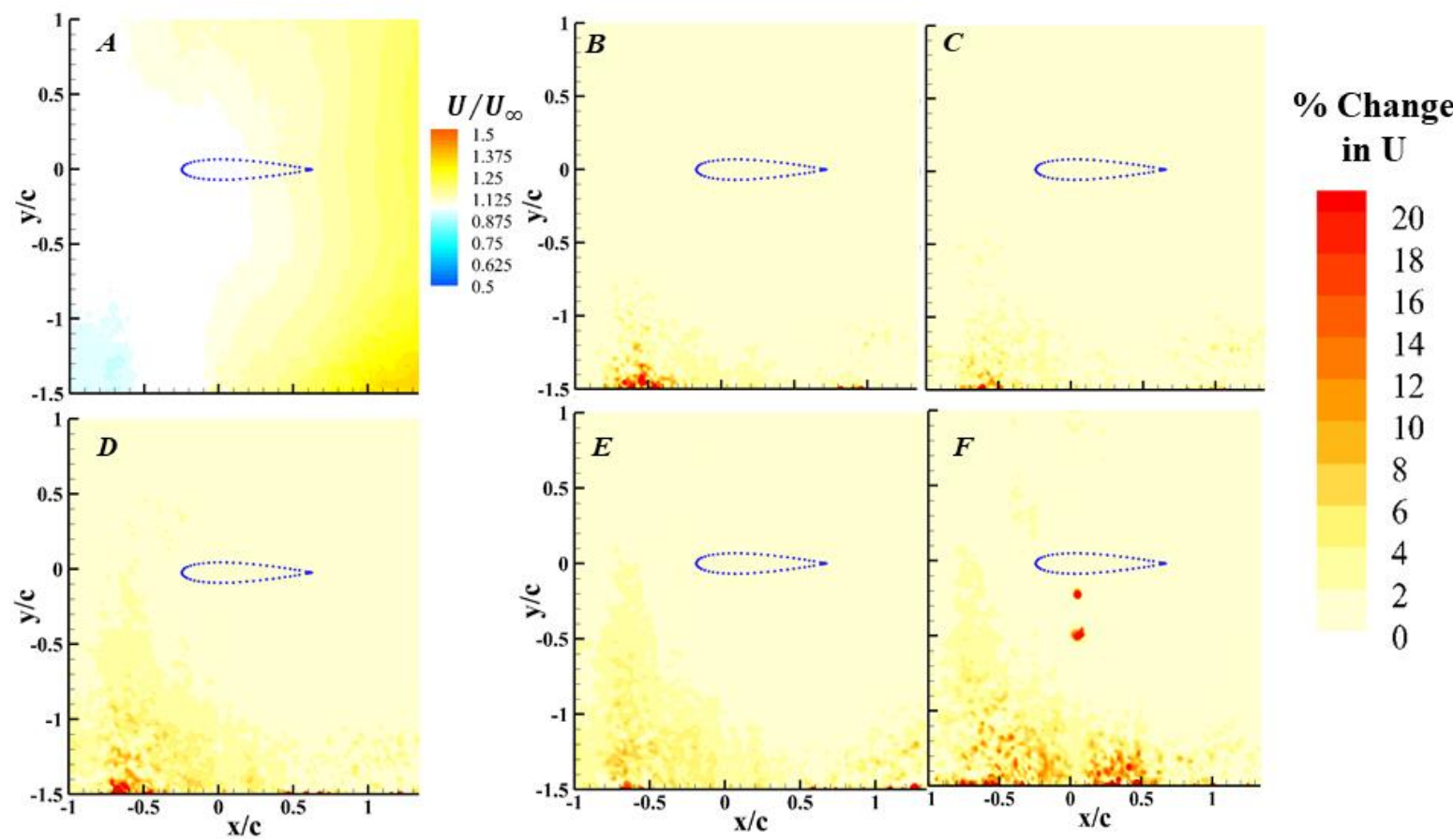

Figure 51: Change in U Velocity based on span location for low power gust.

A) Centerline $V / U_{\infty}$ contour, B) 2" from centerline, C) 4" from centerline, D) 6" from centerline, E) 8" from centerline, F) 10" from centerline.
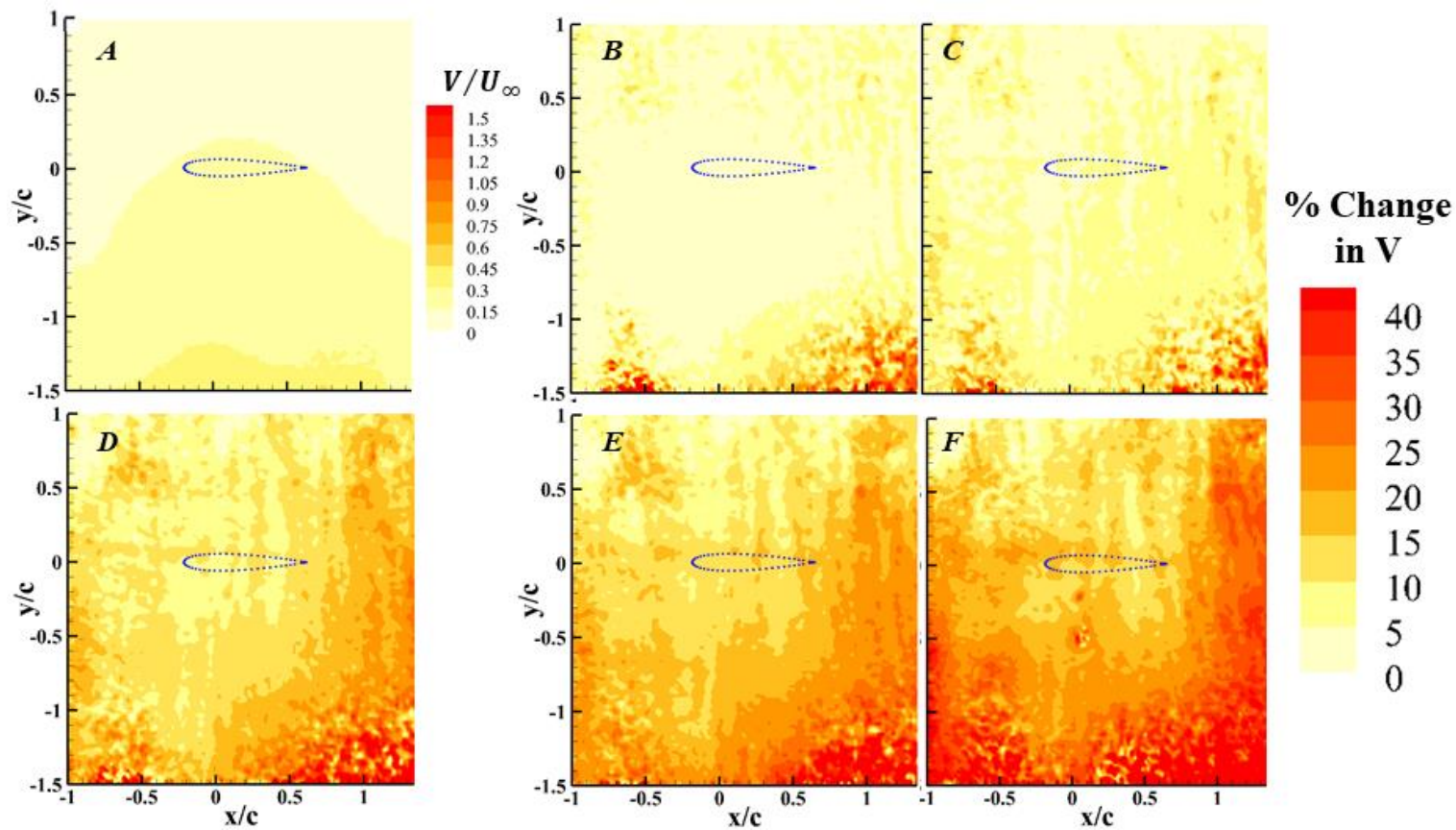

Figure 52: Change in V velocity based on span location for low power gust.

A) Centerline $V / U_{\infty}$ contour, B) 2" from centerline, C) 4" from centerline, D) 6" from centerline, E) 8" from centerline, F) 10" from centerline. 
Quite predictably, the change in $\mathrm{U}$ and $\mathrm{V}$ velocities based on span location are a function of the gust power. As with RMS intensities, the lower the gust power, the lower the value. To minimize drastic changes in $\mathrm{U}$ and $\mathrm{V}$ velocities values based on span and thus minimize 3D effects on the wing, it was best to turn the gust power to the lowest tested setting. This will give a change in $\mathrm{V}$ velocity of only about $5 \%$ at the 4 inches $(0.85 \mathrm{c})$ from the centerline and a change in $\mathrm{U}$ velocity of only $2 \%$ at this point as well. Another interesting point of note is that in all three power cases, there seems to be a jump in velocity changes occurring between the 4 and 6 -inch $(0.85 \mathrm{c}$ and $1.27 \mathrm{c})$ tests. This indicated that something in the geometry of the nozzle at roughly the 5-inch (1.06c) mark is having an impact on the gust being created. As shown in the experimental set up, the nozzle had guide vanes that attempted to help spread the flow evenly along the span. Thus, it is currently assumed that the guide vanes towards the further edge of the jet nozzle are not ideal for the desired spread and should be altered slightly for later revisions. It is quite possible that the angle of the vanes is just too steep and thus the vanes slow the flow. If this proves to be the case, it is suggested that larger holes be used for the outer set of guide vanes when compared to the inner set at the location the nozzle connects to the 90-degree elbow.

\subsection{Chapter Summary}

This chapter explored the capabilities of the gust generator. Characterization was run with just the gust generator on and it was found to produce a gust as desired. Once the tunnel was run in conjunction with the gust generator, the generator was able to produce gust at the wing location with gust ratios between 0.35 and 0.4 . This magnitude 
was well within the initial goal set forth for this prototype design. Finally, the 3D effects present in the flow were explored.

Overall, three main issues with the generator were found. First, the RMS values found in the gust itself were quite high. There were a lot of fluctuations stemming from this design setup. Second, to achieve a maximum gust ratio, the gust would need to be moved further upstream than the tunnel would allow for. Finally, the current gust jet nozzle seemed to be producing a fair amount of 3D effects in the flow. Still, all these issues aside, the gust generator was found to produce a roughly 21-degree flow angle change at the wing location. Therefore, it is believed that this type of generator has significant merits and with some redesign of the current system, the issues can be eliminated, and the gust ratio pushed even higher. 


\section{Chapter 5: Experimental Results}

\subsection{Gust Actuation}

The largest impact a gust has on an MAV is during the first few fractions of a second of their interaction. If the MAV survives this initial interaction, then the remainder of the gust interaction likely will not be of too much concern to the MAV or pilot. Typically, gusts are quick actions that last only a few seconds. This is seen in practically all previous gust-testing experimental apparatuses. Therefore, it is important to determine exactly how the gust created from the generator grows in the tunnel and record this initial interaction. To accomplish this in the current work, the slats on the front of the fan were held closed with an actuator. Upon a command from the Galil controller, the actuator opened the slats and allowed the gust to enter the tunnel. This setup allowed the gust to be started and stopped on command and could better simulate a gust encounter than the fully developed gusts shown in the previous chapter.

This chapter examines the gust actuation at Position 1, as it was previously shown to be the gust location that produced the best gust interaction at the wing location. PIV was acquired and ensemble averaged. The opening of the fan slats was triggered by the camera recording process (sometimes with a fixed time delay) to capture the full gust actuation process in greater time resolution. Throughout this section, the gust strength at the end of the recorded actuation process is compared to the fully developed gust which is the value found from the characterization of the gust in Chapter 4, where the gust was let run for several seconds prior to being recorded and no slats were used. 


\subsubsection{Full Power Phased Gust Actuation}

The behavior of the full-powered gust case is examined in this section. The data shown in this and the remaining sections of this chapter are of various convective times, $t^{*}$, of the flow. Convective time is a non-dimensional unit of time defined here as $t^{*}=$ $\frac{\left(t-t_{\text {gust_start }}\right) U_{\infty}}{c}$, where $\mathrm{t}$ is time at which the frame was recorded (first frame recorded is 0 ), $t_{\text {gust_start }}$ is the time at which the slats on the fan were opened, $\mathrm{c}$ is chord of the wing being considered in later experiments, and $\mathrm{U}_{\infty}$ is defined as the freestream velocity. The change in the horizontal (U) velocity can be seen in Figure 53 as the gust grew to the fully developed case. Only the pre-gust $\mathrm{U}_{\infty}$ value was used in the calculation of $t^{*}$ and the changes in horizontal $(\mathrm{U})$ velocities shown in Figure 53 were not accounted for in the definition of $t^{*}$. Note again that the blue dotted wing is only the planned wing location in the frame of view, no wing was present in these tests.

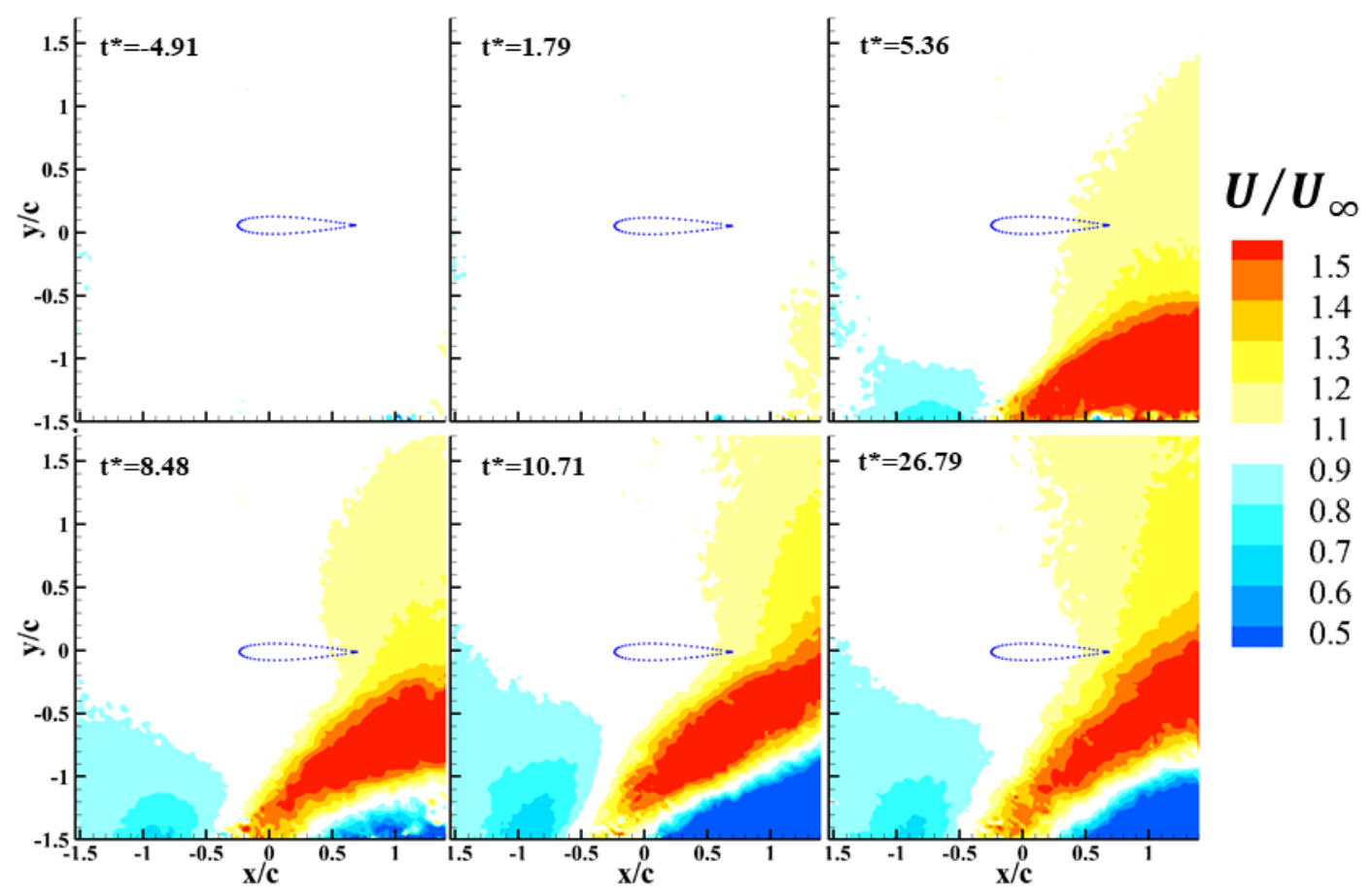

Figure 53: Full-power actuated gust displaying contours of $U / U_{\infty}$ velocity. 
Overall, the results presented in Figure 53 showed growth of the gust from a gustoff condition to a fully developed gust condition observed in the prior characterization. The initial time, $t^{*}=-4.91$, is well before the gust was actuated and no change in freestream was observed. At early times ( $t^{*}=0$ to 4$)$ as the gust entered the tunnel, it was not yet strong enough to alter the flow at the wing location, but some regions of the field of view did experience a change in freestream velocity. Eventually the gust reached a strength at which its impact at the wing location can be seen $\left(t^{*}=5\right)$. This effect slowly grew until the gust was near-fully developed at $t^{*}=10$. Changes in freestream velocity were limited to $10 \%$ near the wing, most of which was near the trailing edge. Throughout the gust actuation process the wing never experienced a decrease in $\mathrm{U} / \mathrm{U}_{\infty}$ velocity; the value only increased throughout the process, again driven by the trailing edge region.

The growth in gust ratio $\left(\mathrm{V} / \mathrm{U}_{\infty}\right)$ is laid out in Figure 54 . It is clearly visible that despite no change in horizontal (U) velocity, there was a positive gust ratio present during the initial time steps, prior to the slats being opened at $t^{*}=0$. It was initially believed that the slats would have kept all of the vertical flow out of the tunnel, but this was not the case. The gust ratio near the wing prior to actuating the gust was approximately 0.14 . The idea of turning off and on the fan to simulate the creation of a gust was tested, but the time it took for the fan to reach full speed was deemed to be too long. Therefore, for this initial prototype design, this leakage was accepted and later corrected for. 


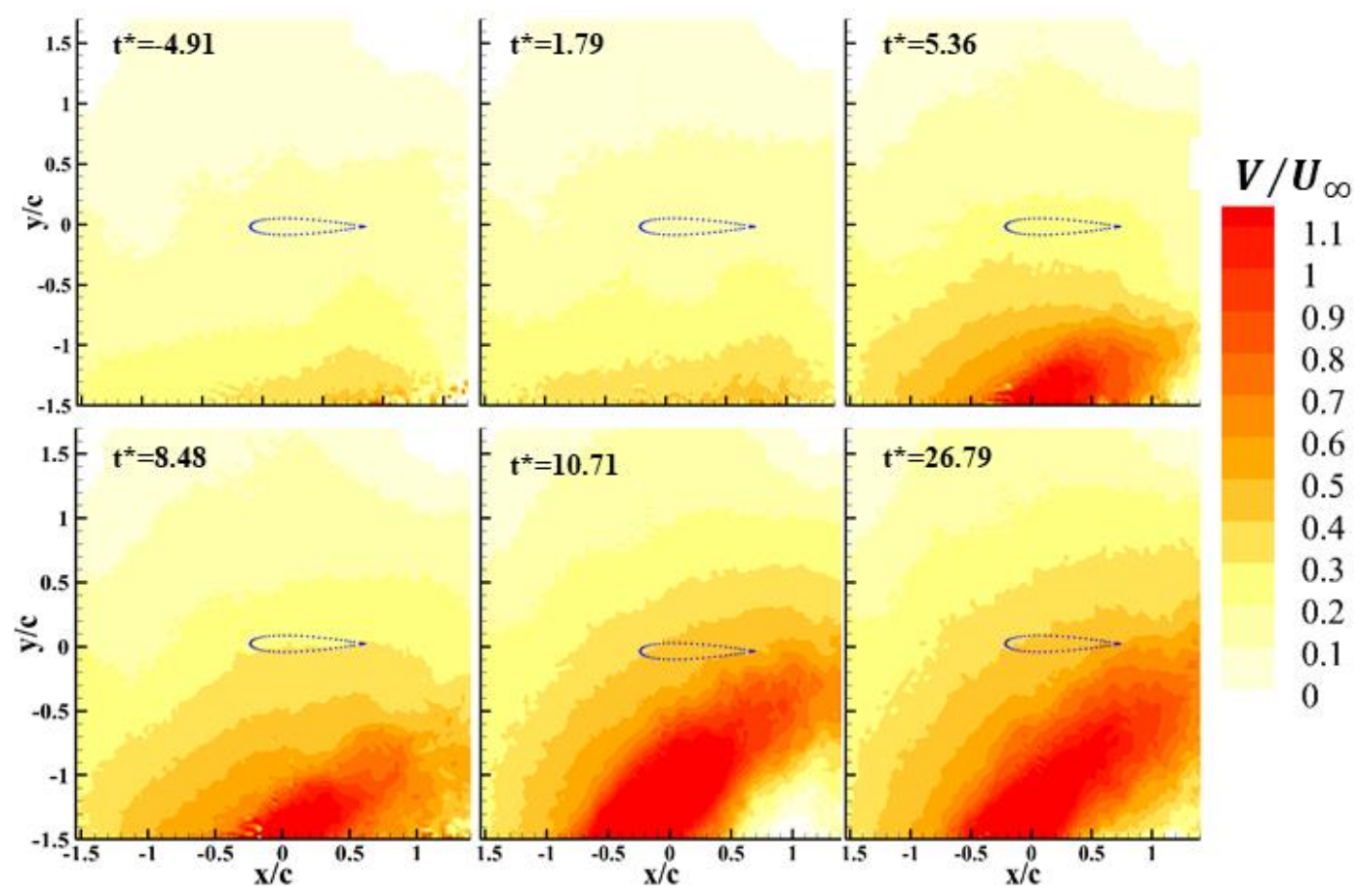

Figure 54: Full power actuated gust displaying contours of gust ratio.

After the gust was actuated it grew in strength with the gust reaching higher and higher into the field of view. Early in the gust development $\left(t^{*}=5.36\right)$ the gust near the wing location rose quickly above $\mathrm{V} / \mathrm{U}_{\infty}=0.2$ and eventually settled at around $\mathrm{V} / \mathrm{U}_{\infty}=0.4$. Throughout the growth of the gust ratio, the gust ratio profile maintained the curved shape seen in the characterization.

The angle of the flow was also calculated through the gust development process. Figure 55 shows the flow angle change across several convective times. The flow angle results from Figure 55 showed the effect of the leakage of flow around the fan slats, with a positive initial flow angle of 8 degrees. However, unlike the previous two gust growth plots, the flow angle grew more as a dome-like shape compared to the bending jet shape of $\mathrm{U} / \mathrm{U}_{\infty}$ and $\mathrm{V} / \mathrm{U}_{\infty}$. This behavior is advantageous compared to other gust generation systems in that the growth of the incoming flow angle was nearly uniform 
across the wing chord. The flow angle grew rapidly reaching a value above 10 degrees by $t^{*}=5.36$ and approaching the peak value above 20 degrees by $t^{*}=10.71$.

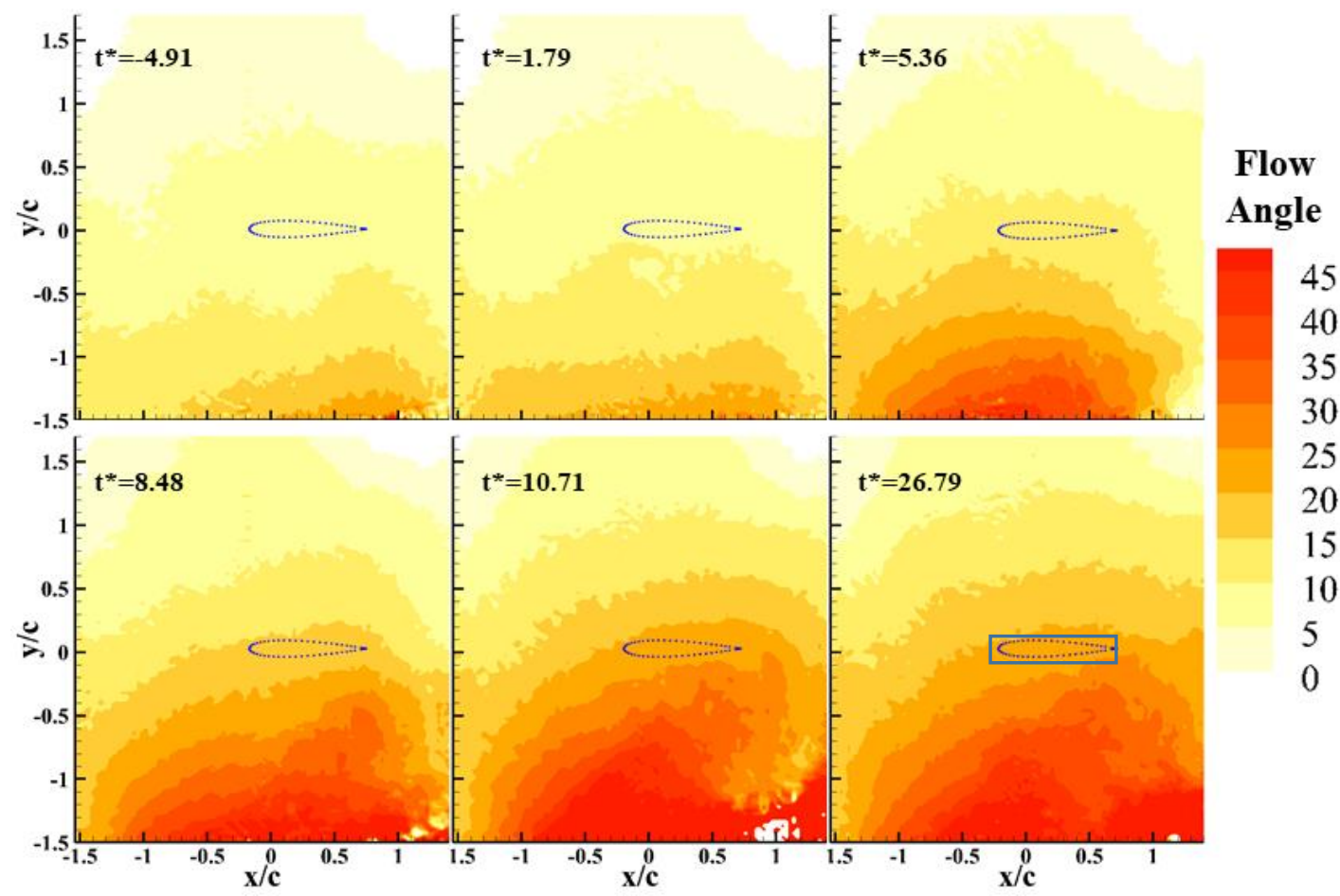

Figure 55: Full power actuated gust contoured to flow angle.

To better visualize these changes in flow properties, data near the wing's planned location was plotted versus time in Figure 56. At each time step, the $\mathrm{U} / \mathrm{U}_{\infty}$, gust ratio, and flow angle data from $\mathrm{x} / \mathrm{c}=-0.25$ to 0.75 and from $\mathrm{y} / \mathrm{c}= \pm 0.06$ were averaged together to create a single data point for that time step. This region can be seen in Figure 55 $\left(t^{*}=26.79\right)$. In Figure 56, these averaged data points are noted in blue, while the red line was created using a built-in MATLAB "Smooth" function and a LOWESS (locally weighted scatterplot smoothing) best-fit line that incorporates the nearest 5 data points. The LOWESS function is a non-parametric regression method. The LOWESS fit curve was used for all discussion of $\mathrm{U} / \mathrm{U}_{\infty}$, gust ratio, and flow angle because it would be impossible to select a single blue data point for analyzing without some bias. 

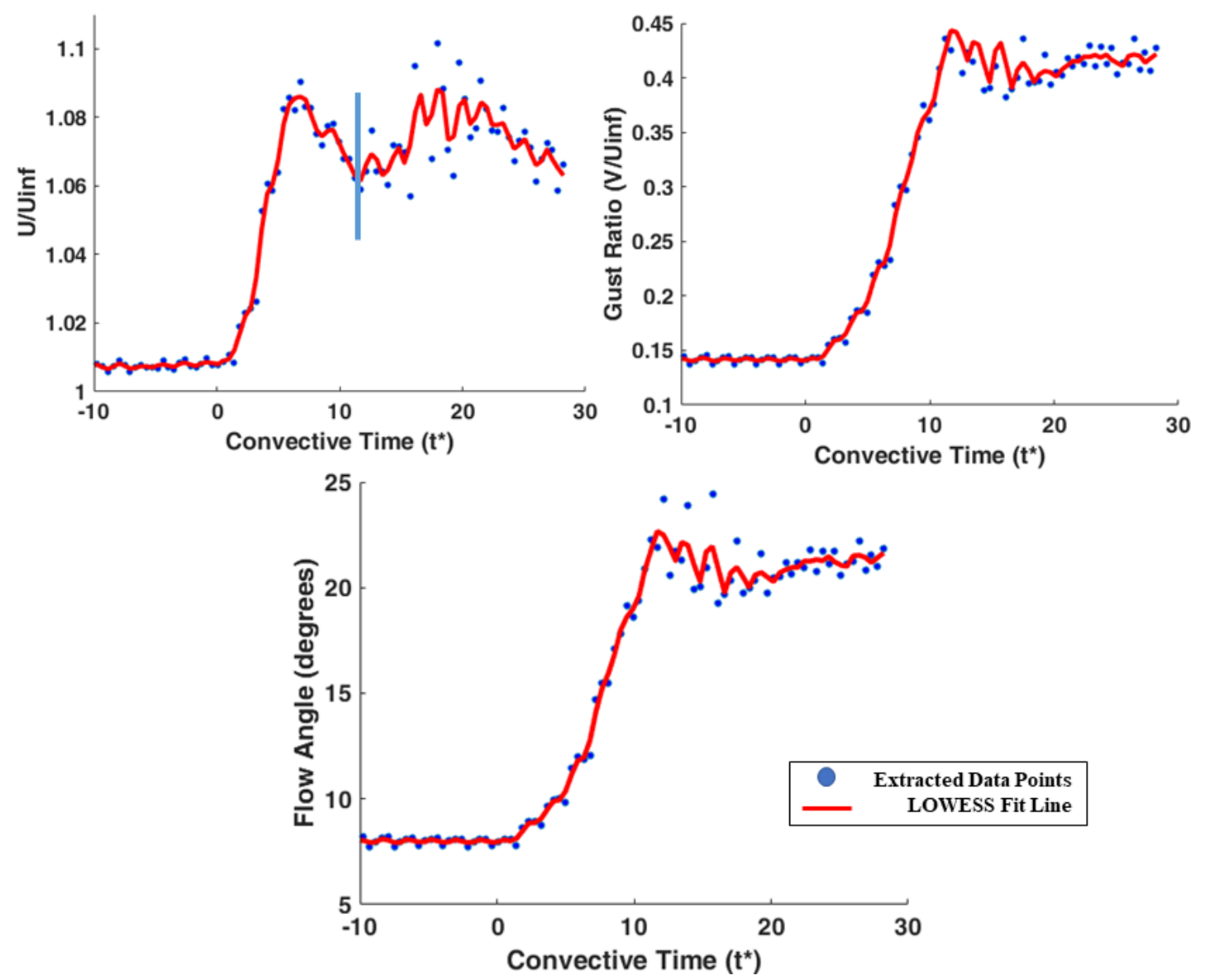

Figure 56: Flow properties vs. convective time for the full power gust case.

The $\mathrm{U} / \mathrm{U}_{\infty}$ data in Figure 56 showed that prior to the slats being opened, the horizontal $(\mathrm{U})$ velocity was already slightly higher than $\mathrm{U}_{\infty}$ by approximately $1 \%$ of freestream. It is likely that the leakage effect from the slats caused this increase much the same way as the gust has been shown to increase the horizontal (U) velocity at a constant full power. It took approximately half a convective time for the gust to start to have an effect on the $\mathrm{U} / \mathrm{U}_{\infty}$ value at the wing's planned location. The $\mathrm{U} / \mathrm{U}_{\infty}$ value reached a maximum of 1.09 at $t^{*}=6.8$.

The gust ratio and flow angle present prior to the opening of the slats were $\mathrm{V} / \mathrm{U}_{\infty}=0.14$ and 8.0 degrees, respectively. These values were higher than desired but were deemed acceptable for the current study based on later corrections. There was an 
observable lag in the change in $\mathrm{V} / \mathrm{U}_{\infty}$ with no change observed until $\mathrm{t}^{*}=1$ at the wing's planned location. The value continued to rise to $\mathrm{V} / \mathrm{U}_{\infty}=0.44$ at $\mathrm{t}^{*}=11.7$ convective times.

Also delayed was the gust's effect on the flow angle near the wing, which wasn't observed to change until $t^{*}=1.5$. Note that the convective times at which the gust seems to affect the measured flow property at the wing location are slightly different in this case. Again, these times were pulled from the LOWESS fit line in a MATLAB window where the user had more control to determine specific values. After this start point, the gust's flow angle also continued to rise until 11.7 convective times. It reached a maximum of 22.66 degrees. It is interesting to note that at $t^{*}=11.7$ convective times (shown via the blue vertical line in the first image of Figure 56), the $\mathrm{U} / \mathrm{U}_{\infty}$ value was actually at a local minimum between its initial peak and a slow oscillation around $\mathrm{U} / \mathrm{U}_{\infty}=1.08$.

As the gust developed, the change in all three calculated values was nearly linear, which is a benefit for any future modeling attempts of this gust generating device. The start and end times of the ensemble averaged data here matched closely to the constant gust results presented in the characterization of Chapter 4. However, after each plot reached these maximum values, there was a small fall off and then the data begins to fluctuate. The largest fluctuation as a percent of the initial value was observed for $\mathrm{U} / \mathrm{U}_{\infty}$. Each fluctuation is found to occur over approximately 0.14 seconds, which is the same frequency at which the camera was run for these experiments, $7 \mathrm{~Hz}$. It is highly unlikely that this is a mere coincidence; but at the same time, it is uncertain how the camera recording rate would have such an effect on the captured data. The data spread outside 
of this oscillation is attributed to the turbulence seen in the RMS values calculated for the flow. The flexible ducting used to pump the gust into the nozzle at the bottom of the tunnel was observed oscillating throughout the tests. It is thought that this oscillation, along with the use of a non-research grade fan with limited flow straightening, resulted in the spread of data visible in Figure 56.

\subsubsection{Mid and Low Power Phased Gust Actuation}

Data for mid and low fan power cases was collected but for brevity, these results are shown in Appendix B. The flow quantities grew much the same as the full power case, but with lower strengths. The gust also took longer to develop, and the ensemble averaged data acquisition process was not long enough to capture the full development of the gust in many of the cases. The results are summarized by plotting the extracted flow data $\left(\mathrm{U} / \mathrm{U}_{\infty}\right.$, gust ratio, flow angle) at the wing position versus convective time. These results for the mid power case are shown in Figure 57, and for the low power case in Figure 58. 

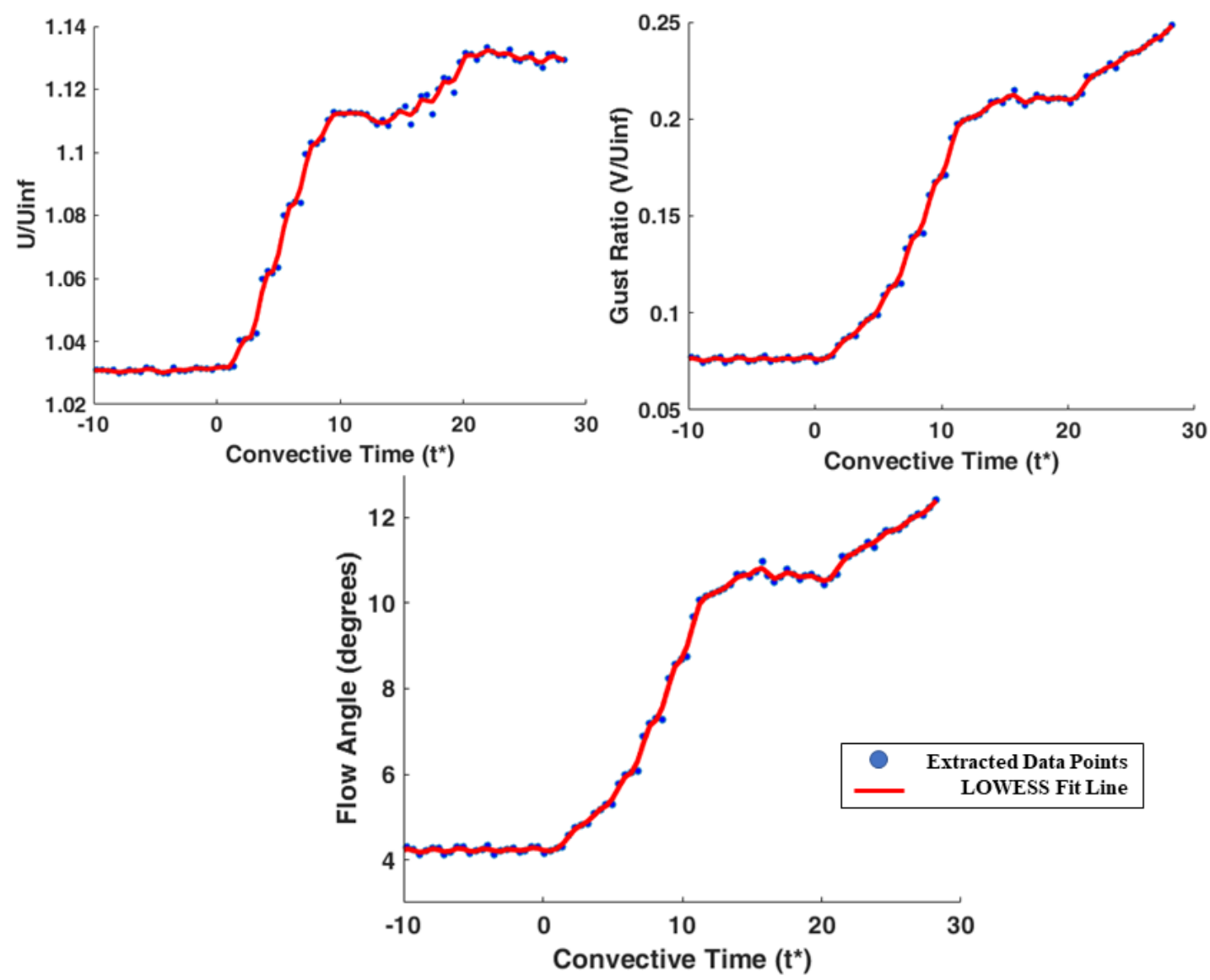

Figure 57: Flow properties vs. convective time for the mid power gust case.

The plots in Figure 57 show a similar trend to the full-power case for the first 10 convective times but deviated a fair bit after that. The initial, pre-gust, values are shown to be $1.03,0.08$, and 4.25 for the $\mathrm{U} / \mathrm{U}_{\infty}$, gust ratio, and flow angle respectively. Furthermore, the gust's effect can be seen to reach the wing's location around $t^{*}=1.0$ for all three components. From there, the $\mathrm{U} / \mathrm{U}_{\infty}$ value climbs to a local maximum of 1.11, which occurs at $t^{*}=9.45$. This local maximum value for $\mathrm{U} / \mathrm{U}_{\infty}$ is roughly as was shown by the constant gust characterization. However, it was difficult to determine exactly where the maximum points lie for all three data sets. There was no drop off as the gust settled to its constant strength at long times as there was in the full power case. 
In fact, the plots show that the $\mathrm{U} / \mathrm{U}_{\infty}$ data continues to increase after a very brief settling period. Therefore, these initial maxima were taken as the $t^{*}$ value where the slope of the initial growth region clearly begins to decrease. After this point, the $\mathrm{U} / \mathrm{U}_{\infty}$ value continues to increase at a slower rate, but eventually settles around $t^{*}=20.2$ to a value of 1.13 , which is slightly higher than predicted in the characterization for the constant gust.

The gust ratio for the mid-power case climbs to a local maximum of 0.20 at $t^{*}=11.7$. The flow angle climbs to a local maximum of 10.15 degrees also at $t^{*}=11.7$. When compared to the fully developed gust values as found in the characterization from Chapter 4, these local maxima for gust ratio and flow angle are slightly less for the actuated gust. However, during the second growth period, the gust ratio reaches the same value found during the constant gust characterization, but the data was not recorded long enough to determine if it would pass this point prior to settling. The flow angle does not reach the value found in the fully developed characterization within the data recorded. It is not currently known why the gust ratio and flow angle in this midpower case do not settle by the end of the recording. Since the gust is created with a lower fan power than was tested in the full power-case, it is assumed that the influence of the gust begins further downstream and lower in the test section, thus the effects will creep forward into the measurement zone over a longer time. However, due to experimental time constraints, data acquisition was not repeated to determine exactly how long it takes the flow angle and gust ratio to reach steady values. The long-time values for these properties can be extrapolated based on the constant gust results from the characterization; however, unless it is assumed that the slope of this secondary 
growth region is constant, the time at which the actuated gust reaches these fully developed gust values cannot be determined.

It can also be seen that compared to the full power gust case, there is far less fluctuation in the later time steps of the $\mathrm{U} / \mathrm{U}_{\infty}$ values, but the same frequency of $7 \mathrm{~Hz}$ can still be seen in the small fluctuations present. The decrease in data spread is attributed to the decrease in flow RMS seen in the characterization of the gust at lower powers.

The final empty tunnel experimentation that was completed was for the gust actuating at low power. The graphs showing the same plots for the low power gust case can be seen in Figure 58.
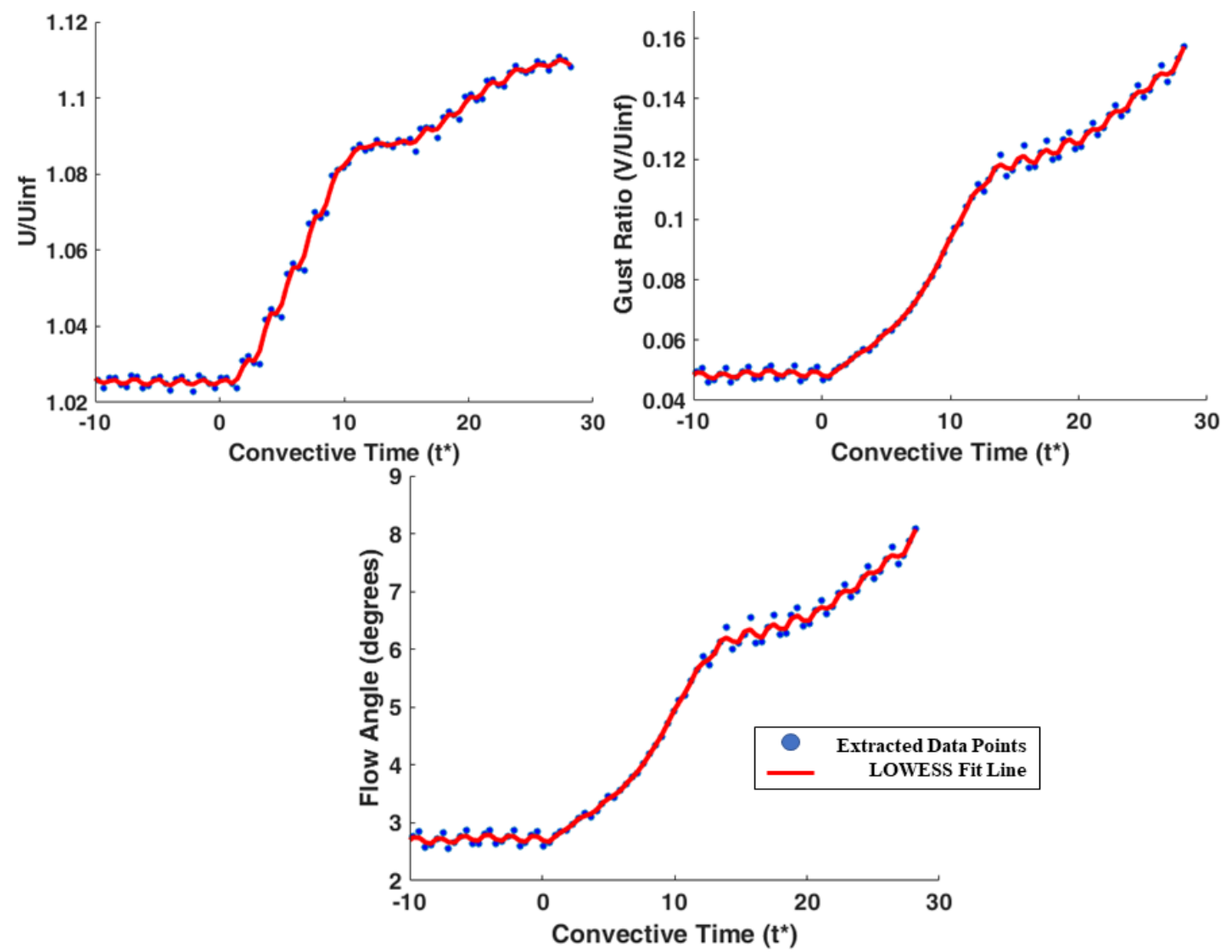

Figure 58: Flow properties vs. convective time for the low power gust case. 
The initial values of the $\mathrm{U} / \mathrm{U}_{\infty}$, gust ratio, and flow angle are 1.03, 0.05, and 2.72 respectively. For the $\mathrm{U} / \mathrm{U}_{\infty}$ plot, the gust's effects can be seen at the wing's position at 1.4 convective times after the slats open. As before, a sharp increase is seen to start to form at this point. However, the initial "peak" of the $\mathrm{U} / \mathrm{U}_{\infty}$ plot, as well as the other properties is less discernible, even less so than the mid power case. A small flattening does occur in the graphs, but does not last for long. Thus, the initial local peak was again determined as the point at which the slope of the initial climbing region decreased. This point was determined to be at $t^{*}=9.4$ for $\mathrm{U} / \mathrm{U}_{\infty}$, at which point the value was estimated to be 1.08 . However, as with the mid power case, this value continued to increase after this initial leveling point to a value of 1.11 , at which point the data acquisition terminated without capturing the fully developed flow.

In the low power case, the gust ratio change became noticeable at $t^{*}=1$. The initial leveling seen for the gust ratio occurs at $t^{*}=12.1$ at which point the value is 0.11 . It then continues to grow to 0.16 . The flow angle change began to occur at 0.8 convective times. The flow angle grows to an initial peak of 5.76 at $t^{*}=12.1$. It then continues to grow to about 8 degrees.

At this lower power the oscillations in each of the properties become slightly more discernible when compared to the mid power case. It is clear here that these oscillations are at a constant frequency, which was again calculated to be $7 \mathrm{~Hz}$. However, the data spread is again quite low showing the same drop in RMS value at this low power setting seen in the characterization of the fully developed gust at this power. 
To better quantify and compare the changes seen in each case, several tables are laid out that gather the properties discussed in one location. "Before Gust" represents the value of the component prior to the actuation of the slats. The "Initial Peak" is either the peak value recorded prior to settling as in the full power gust case, or the value at which the inflection point discussed for the mid and low power gust cases was observed. The "End" is the average value of the fully developed gust's fluctuations seen for the full power case, or the value of data captured in the final frame of the recording for the mid and low power gust cases. The "Fully Developed Gust" is the value found in Chapter 4 during the characterization of each component.

Table 5: U/U $U_{\infty}$ values across the actuation.

\begin{tabular}{|c|c|c|c|c|}
\hline & $\begin{array}{c}\mathrm{U} / \mathrm{U}_{\infty} \text { Before } \\
\text { Gust }\end{array}$ & $\begin{array}{c}\mathrm{U} / \mathrm{U}_{\infty} \text { Initial } \\
\text { Peak }\end{array}$ & $\mathrm{U} / \mathrm{U}_{\infty}$ End & $\begin{array}{c}\text { U/U } \mathrm{U}_{\infty} \text { Fully } \\
\text { Developed } \\
\text { Gust }\end{array}$ \\
\hline Full Power & 1.01 & 1.09 & 1.07 & 0.98 \\
\hline Mid Power & 1.03 & 1.11 & 1.13 & 1.03 \\
\hline Low Power & 1.03 & 1.08 & 1.11 & 1.06 \\
\hline
\end{tabular}

Table 6: $V / U_{\infty}$ values across the actuation.

\begin{tabular}{|c|c|c|c|c|}
\hline & $\begin{array}{c}\mathrm{V} / \mathrm{U}_{\infty} \text { Before } \\
\text { Gust }\end{array}$ & $\begin{array}{c}\mathrm{V} / \mathrm{U}_{\infty} \text { Initial } \\
\text { Peak }\end{array}$ & $\mathrm{V} / \mathrm{U}_{\infty}$ End & $\begin{array}{c}\mathrm{V} / \mathrm{U}_{\infty} \text { Fully } \\
\text { Developed } \\
\text { Gust }\end{array}$ \\
\hline Full Power & 0.14 & 0.44 & 0.42 & 0.38 \\
\hline Mid Power & 0.08 & 0.20 & 0.25 & 0.28 \\
\hline Low Power & 0.05 & 0.11 & 0.16 & 0.15 \\
\hline
\end{tabular}

Table 7: Flow angle values across the actuation.

\begin{tabular}{|c|c|c|c|c|}
\hline & $\begin{array}{c}\text { Flow Angle } \\
\text { Before Gust }\end{array}$ & $\begin{array}{c}\text { Flow Angle } \\
\text { Initial Peak }\end{array}$ & $\begin{array}{c}\text { Flow Angle } \\
\text { End }\end{array}$ & $\begin{array}{c}\text { Flow Angle } \\
\text { Fully } \\
\text { Developed } \\
\text { Gust }\end{array}$ \\
\hline Full Power & 8 & 22.66 & 21 & 21.1 \\
\hline Mid Power & 4.25 & 10.15 & 12.60 & 15.1 \\
\hline Low Power & 2.72 & 5.76 & 8.02 & 8.02 \\
\hline
\end{tabular}


While all the cases in Table 5 showed a rising $\mathrm{U} / \mathrm{U}_{\infty}$, the final value in the ensemble average was consistently higher than the full-developed gust results from the characterization. This suggests that there was some further variation in the flow past the maximum $\mathrm{t}^{*}$ measured, although that variation was somewhat minor for $\mathrm{U} / \mathrm{U}_{\infty}$. noted here.

Table 6 and Table 7 follow a trend as would be expected, with the full power case creating the largest gust ratio and flow angle changes, followed by the mid and low power cases, respectively. The comparison between the mid and low power cases to the fully developed gust from the previous chapter shows that the gust is quickly approaching the characterized values and may only be a few convective times short of reaching the fully developed case.

Table 8 extracts the convective times from Table 5 through Table 7 at which some of the highlighted flow property values occur. "Start" is defined as when the gust effect on the measured property reaches the wing location. "Peak" is defined as the time at which the measured property reaches the "Initial Peak" value shown in the above tables. The "Start" values for $\mathrm{U} / \mathrm{U}_{\infty}$ varied somewhat linearly as a function of gust power condition. Peak $\mathrm{U} / \mathrm{U}_{\infty}$ occurred early for the full-power case but was observed to be a few convective times later for low and mid power cases.

The values and times for the gust ratio and flow angle are much more precise. Using these values for the full power gust, the gust takes about 10.7 convective times to fully actuate. This is a bit slower than previous gust experiments, which typically aim for approximately 6 or fewer convective times [73] [75]. It is still deemed fast enough to be a valid representation of some environmental gusts. 
Table 8: Selected $t^{*}$ values from actuation process.

\begin{tabular}{|c|c|c|c|c|c|c|}
\hline & $\begin{array}{c}\mathrm{U} / \mathrm{U}_{\infty} \\
\text { Start }\end{array}$ & $\begin{array}{c}\mathrm{U} / \mathrm{U}_{\infty} \\
\text { Peak }\end{array}$ & $\begin{array}{c}\mathrm{V} / \mathrm{U}_{\infty} \\
\text { Start }\end{array}$ & $\begin{array}{c}\mathrm{V} / \mathrm{U}_{\infty} \\
\text { Peak }\end{array}$ & $\begin{array}{c}\text { Flow } \\
\text { Angle } \\
\text { Start }\end{array}$ & $\begin{array}{c}\text { Flow } \\
\text { Angle } \\
\text { Peak }\end{array}$ \\
\hline $\begin{array}{c}\text { Full } \\
\text { Power }\end{array}$ & 0.5 & 6.8 & 1.5 & 11.7 & 1.0 & 11.7 \\
\hline $\begin{array}{c}\text { Mid } \\
\text { Power }\end{array}$ & 1 & 9.45 & 1 & 11.7 & 1 & 11.7 \\
\hline $\begin{array}{c}\text { Low } \\
\text { Power }\end{array}$ & 1.4 & 9.4 & 1 & 12.1 & 0.8 & 12.1 \\
\hline
\end{tabular}

The evolution of the gust to its fully developed state was evaluated using an ensemble average of data which was timed based on the gust being actuated. For the full power case, a peak gust angle of 22.7 degrees was reached in 11.7 convective times, but some oscillations were observed in the flow after that peak. The peak angle and development rate of the gust were both reduced at lower fan powers. Flow angle was observed to grow near-uniformly along the chord which is a unique feature of the current gust generation system. After this analysis, the full power gust was identified as the best use-case for the gust generator. The lower powers were still evaluated in further testing, but with acknowledgement that the gust was not fully developed during the ensemble average.

As stated, it is very important to know how the gust grew over time as the slats were actuated. As the primary focus of the remaining results will pertaining to how this actuation effects a wing, it is good to have a baseline of these results for an empty tunnel case. Using these results, estimated flow angles about the wing at various gust strengths and convective times were determined and used for comparisons throughout the remainder of this thesis. 


\subsection{Gust-Wing Interaction}

After the gust was fully characterized for actuation, a wing was placed in the tunnel to observe the effects the gust would have on the wing. In this section, the data will be largely displayed in terms of flow fields with nondimensional vorticity. This was done to identify any repeatable vortex structures which are common in other gust studies [73] [75]. In order to isolate the effects of the gust-wing interaction on the voriticity, it was important to see if any vorticity was present in the flow prior to the wing being installed. Figure 59 shows the data displaying contours of vorticity for the constant strength gust at full power and Position 1. The entire frame of view appears to be white indicating from the contour bar that all nondimentional vorticity is between 4 and 4 . From this result, it is clear that any vorticity present in the flow is quite low and therefore can be largely ignored for the future data sets. There was also little vorticity present for the lower fan power cases. Thus, any vorticity visible in the future plots can be assumed to come from the wing's interaction with the flow.

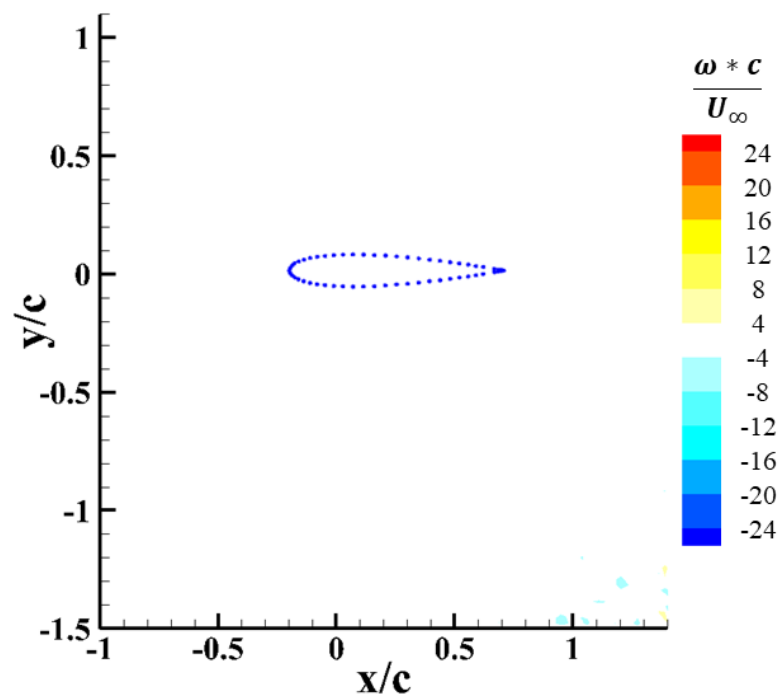

Figure 59: Vorticity contour for the gust at full power in an empty tunnel. 
There was a desire to test the wing in the gust at several angles of attack to better understand what an MAV may experience in flight. Ideally, the wing would be tested for gust interaction at 0,5 , and 10 degrees. The issue with this testing goal was the initial flow conditions present prior to the gust actuation. The initial flow angle in the tunnel prior to actuating the gust was non-zero. To test at the desired wing angles of attack (AoA), the flow needed to be corrected in some manner. For this study, an effective AoA correction was done and evaluated for its effectiveness.

The method for calculating effective angle of attack can be seen in Figure 60, where the components of the total velocity vector seen by the wing are separated. The total effective angle of attack was defined as a function of the geometric AoA and the AoA induced by the gust actuation.

In addition to the vertical component of velocity induced by the gust, it is important to remember that the gust also affected the horizontal flow velocity, although significantly less than the vertical component. The combined gust and freestream velocity components allow one to find the flow angle, as has been done several times in this report. The effective angle of attack is therefore simply the total flow angle added to the geometric angle of attack of the wing.

Selecting a single flow angle for the wing as a whole can become difficult since each chordwise segment of the wing might be experiencing different flow conditions. For many experiments where effective angle of attack is important, the effective flow angle is usually measured at the leading-edge. However, in this case the effective flow angle was determined as the average of the angles seen by the entire wing. This method was preferred to the leading-edge measurement because the flow angle data captured 
in the ensemble average, Figure 55, showed that the flow angle grew almost uniformly along the wing chord.

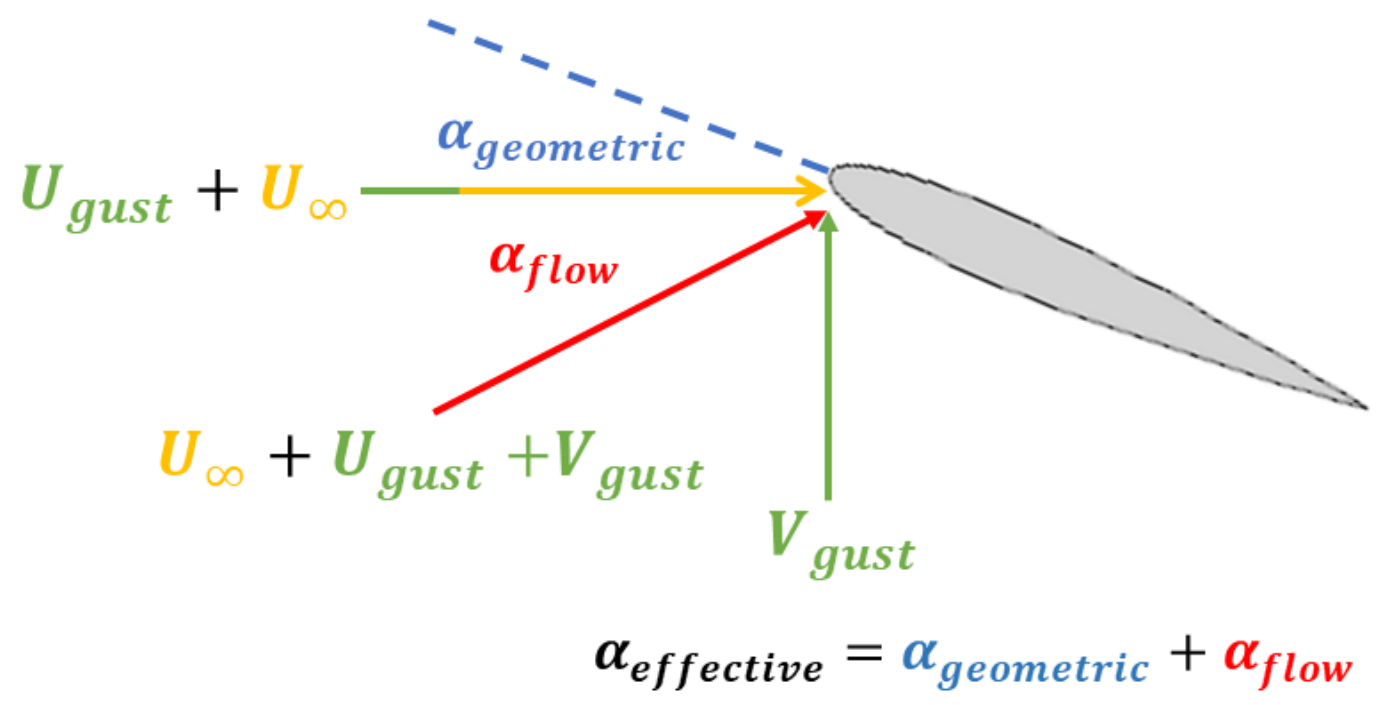

Figure 60: Effective angle of attack in a gust.

\subsubsection{Full Power Gust-Wing Interaction, 0 Degrees}

After identifying a possible correction for the pre-actuation flow angle present in the tunnel, the wing was pitched down 8 degrees to set the effective AoA to 0 degrees. Gust interactions were observed first at a steady flight condition of 0-degree effective angle of attack. For vector remapping and comparison purposes the data in this study is most commonly shown in the wing frame of reference. Figure 61 shows the flow field during gust actuation at full power with the wing in place for a 0-degree effective angle of attack. Estimated incoming flow angles were included for analysis (a). These estimated incoming flow angles were calculated based on the empty tunnel flow angles seen at the noted convective times in the actuation characterization, Figure 56. 


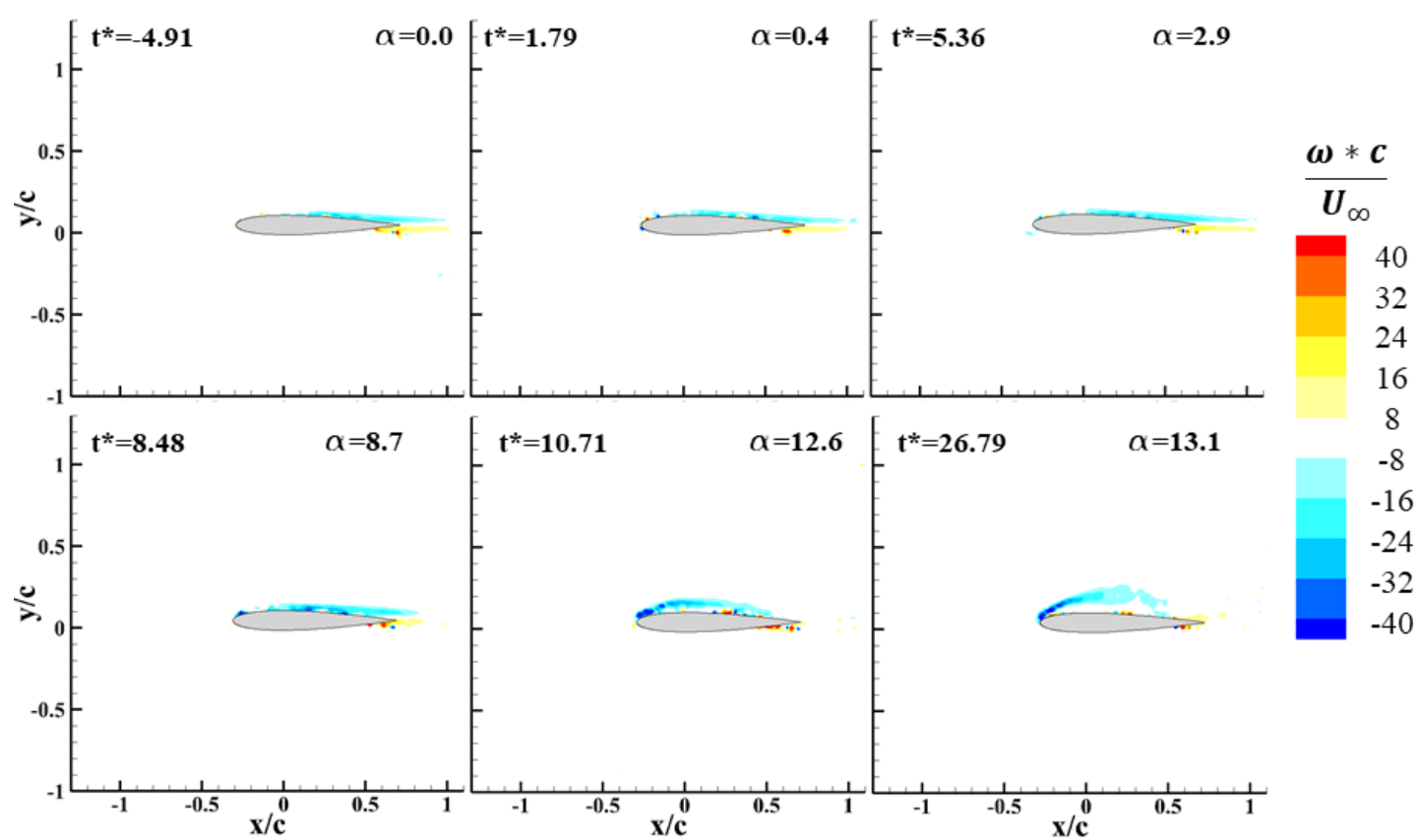

Figure 61: Actuating full power gust; wing at 0-degree initial effective angle of attack.

During the initial interaction of the gust and the wing, the top and bottom boundary layers from the wing were observed to separate from the trailing edge evenly and with no notable deflection angle. This suggests that the effective angle of attack correction was sufficient to adjust for the flow present prior to the actuation of the gust.

The flow remained attached to the wing and without notable changes in the boundary layer around the wing until after $t^{*}=5.36$. During the ensemble average characterization, the flow angle was more than halfway through its growth at this point in time and was quickly approaching the fully developed gust condition. When looking at contours of vorticity, it appears to take a bit longer for the gust's effects to be seen. This is because any changes near the leading edge of the wing, where the majority of vorticity is shed, have to convect downstream to change the upper surface flow, so it naturally takes longer for the gust's effects to be seen with a wing present and displaying vorticity in the figures. 
A new series of $t^{*}$ values were selected to better show the flow evolution in Figure 62. Streamlines were also added to help further analysis of the flow.

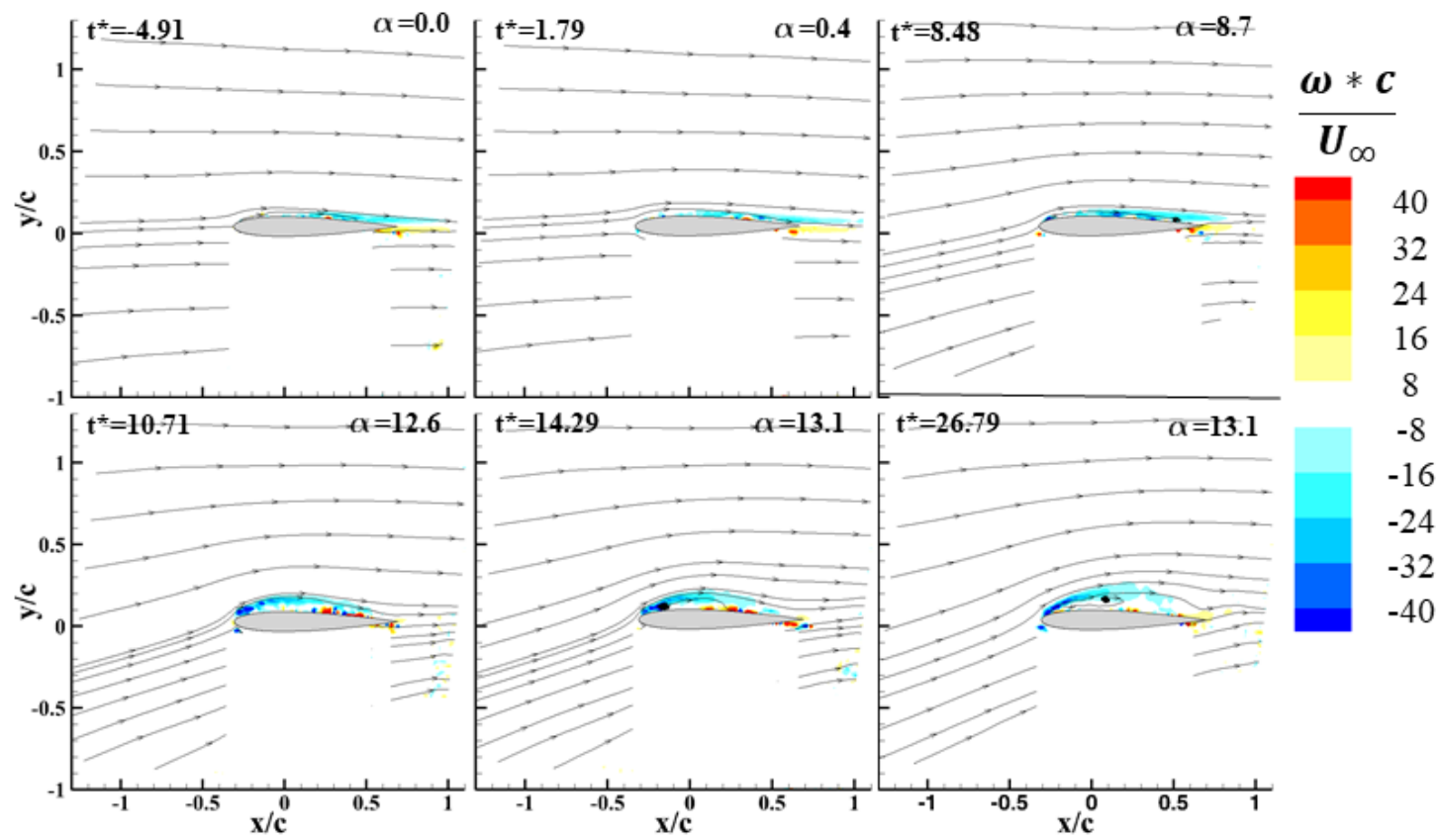

Figure 62: Actuating full power gust; wing at 0-degree initial effective, new timesteps.

As the gust grew through $\mathrm{t}^{*}=10.71$, a separation bubble formed on the wing, but flow remained attached at the trailing edge. At around $t^{*}=14.29$, the bubble grew to the point where the flow separated from the trailing edge and the wing entered a fully stalled condition. It is shown that at $t^{*}=14.29$, the wing is at an estimated 13.1-degree effective angle of attack, which at this low of a Reynolds number is well past the known stall AoA for a NACA 0012.

Clearly there was still growth occurring in vorticity at $t^{*}=14.29$; however, the streamlines show the flow direction has stopped changing by then. As stated, this lag in vorticity growth was expected. After analyzing the growth of the vorticity from the full data set timestep by timestep, it was found that the steady state for vorticity was reached around $\mathrm{t}^{*}=15.7$, about 4 convective times after the flow properties reached a 
fully developed state in empty tunnel actuation characterization from section 5.1. It was hypothesized that any force change on the wing from this gust interaction would also have this lag effect.

The estimated effective angles of attack $(\alpha)$ were assigned to the frames based on the known flow angle found in the empty tunnel gust actuation characterization from the section 5.1. From Figure 56, the angle of the flow at a given convective time was found. 8 degrees was subtracted from the values found at specific convective times because of the wing's initial -8-degree pitch angle and the remainder was taken as the hypothesized effective angle of attack. This hypothesized effective angle does not change much over the last 3 frames shown, but clearly the vorticity was still changing fairly significantly across these frames.

\subsubsection{Full Power, 0 Degree Effective AoA Case Compared to Static Wing}

Vorticity growth and stall by a wing is a well-known result of a wing experiencing higher and higher angles of attack; so, a simple comparison between the wing-in-gust case and a static wing at various angles of attack was made. Measurements were taken from 0 to 35 degrees with 5-degree increments for a stationary wing for these comparisons. The results for these stationary wing cases can be seen in Figure 63 and Figure 64. These figures present the same data, only Figure 64 is rotated to the wing's frame of reference and thus it appears to be at 0 degrees in the frame of view. Data for the 30 and 35-degree cases can be seen in the appendix as they were not used for comparisons in this thesis. 


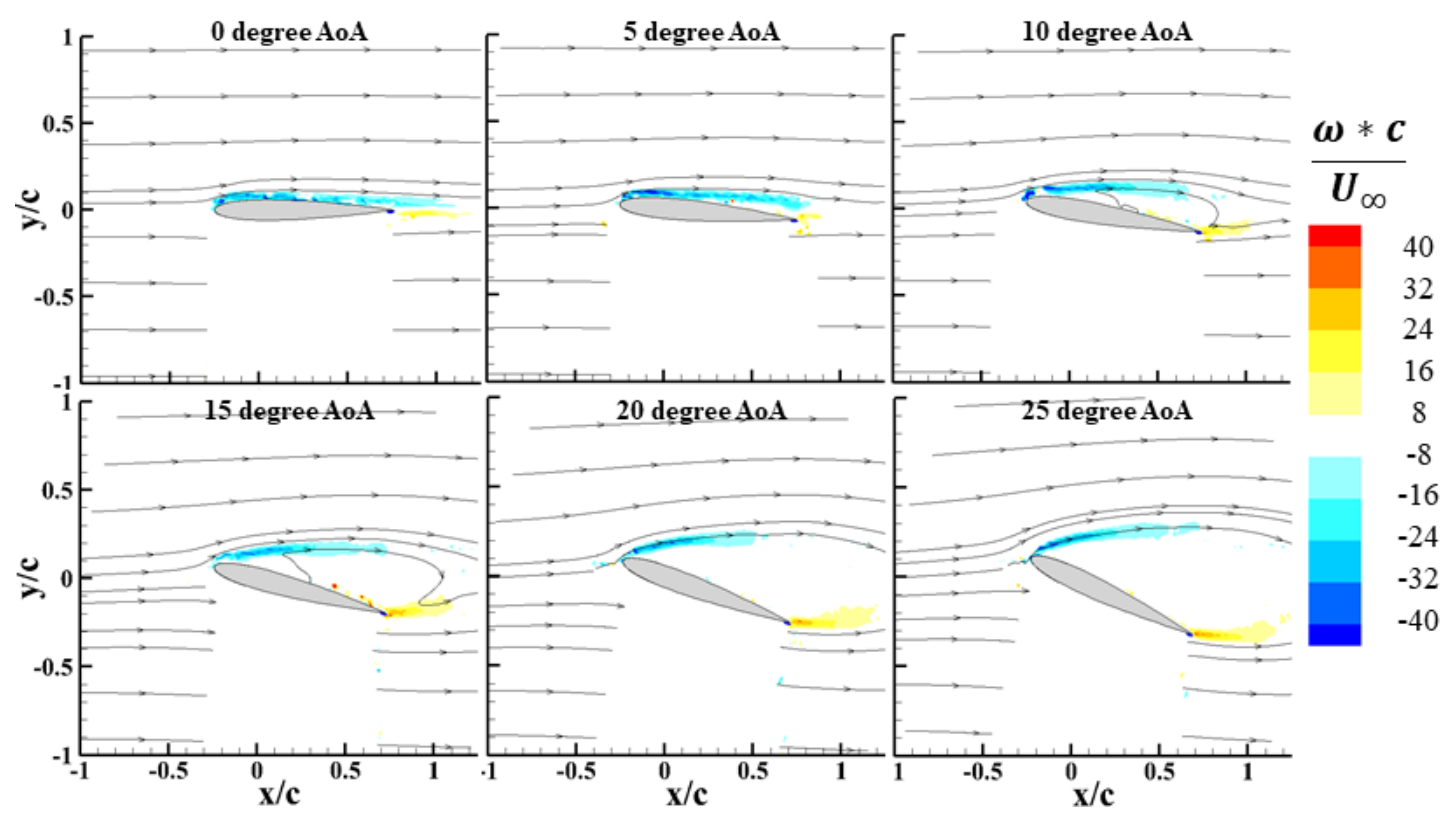

Figure 63: Static wing at positive angles of attack in lab frame of reference.

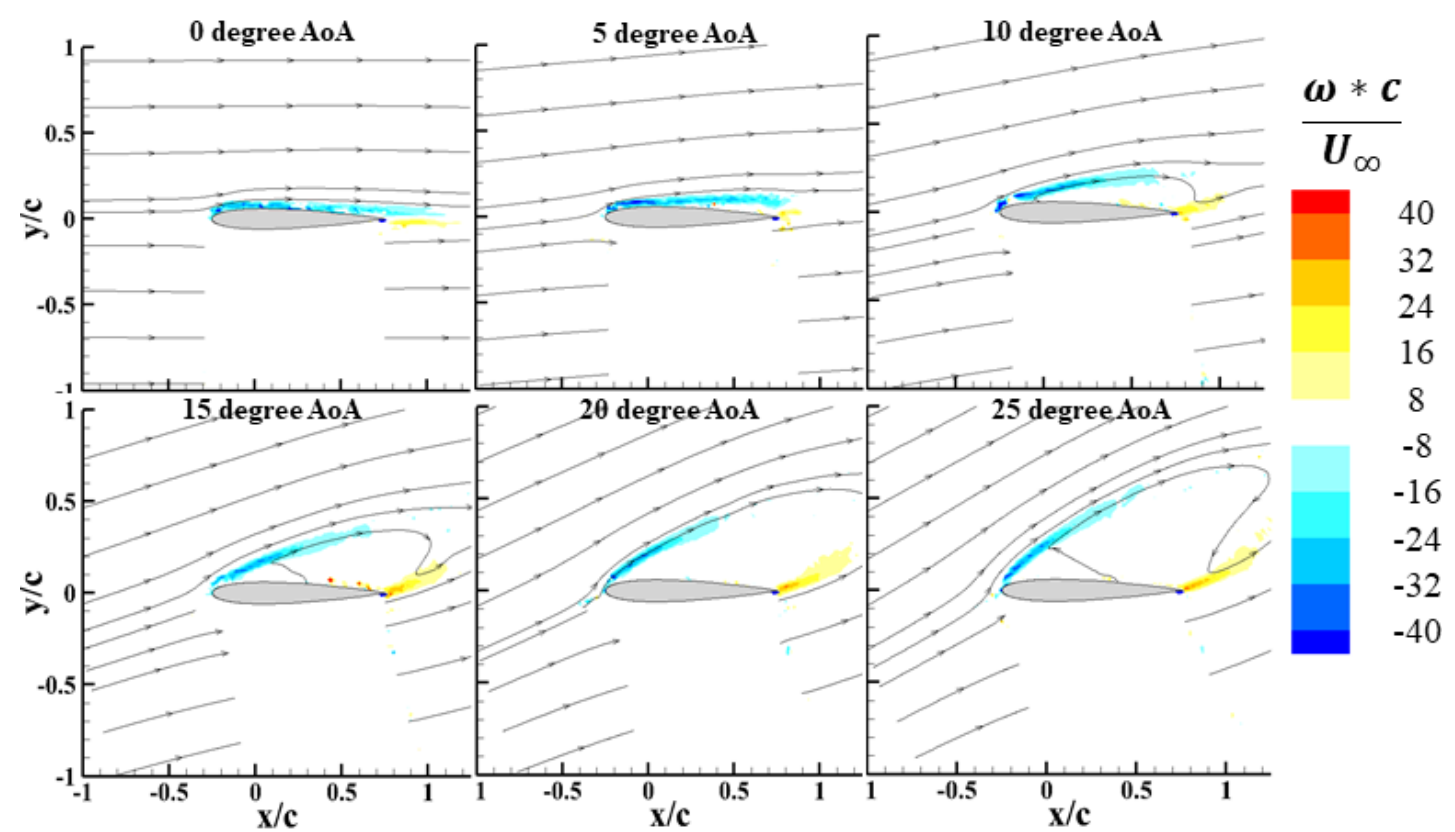

Figure 64: Static wing at positive angles of attack in wing frame of reference.

Once these data sets were taken, there was a desire to see how closely the various points in the actuated gust flow cases compared to some wing steady state values. This would help to show exactly what similarities and differences existed between the effective angle of attack due to a gust compared to a simple angle of attack change of 
the wing. The first check was to see if the 0-degree effective angle case for the wing in the gust-slat leakage matched the actual 0-degree angle of attack case. These results can be seen in Figure 65.

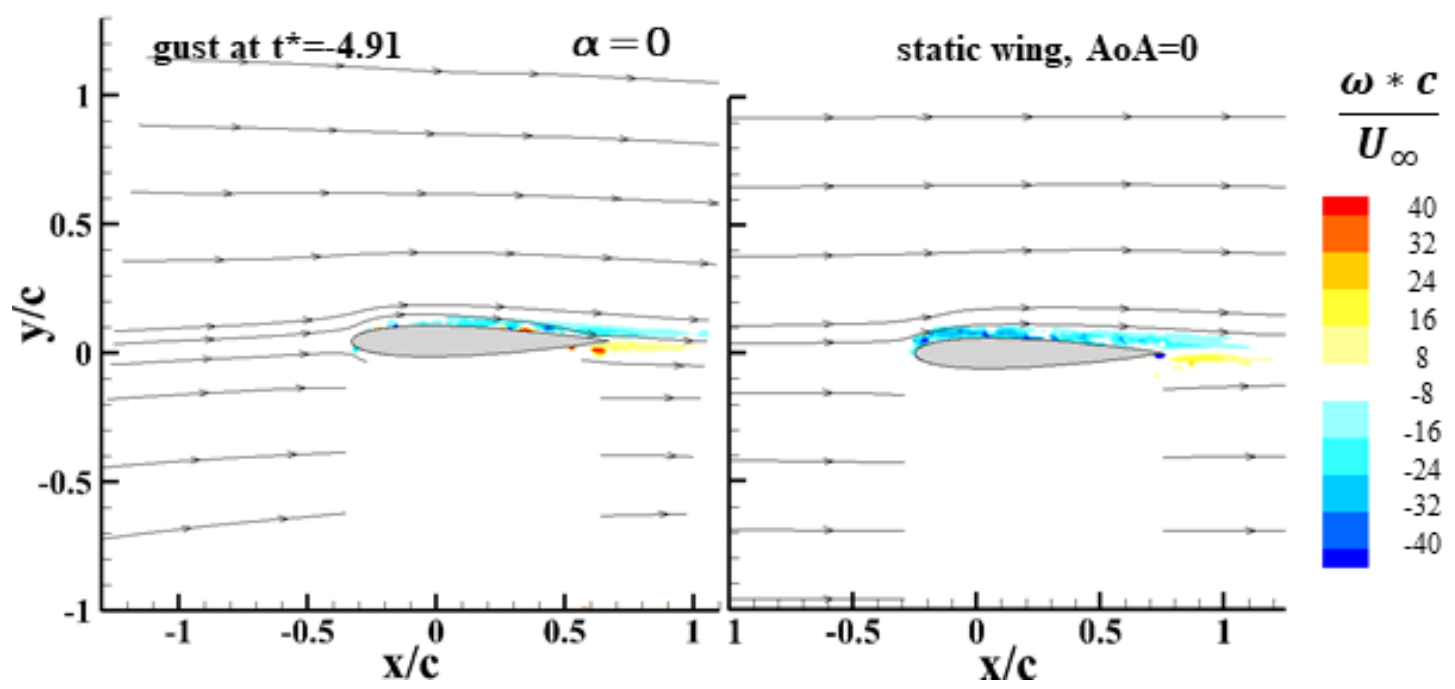

Figure 65: Pre full-power-gust/static wing comparison; 0-degree effective angle.

Overall there was significant correlation between the two cases. The vorticity of both cases was fully attached to the wing and sheds from the trailing edge in similar fashions. Had the laser sheet been projected on the bottom of the wing, it is highly likely that equal vorticity would be seen in these cases on the bottom surface based on the positive shed vorticity captured at the trailing edge. The streamlines in the two images also matched closely. However, a small change in their direction throughout the field of view for the gust case was seen and can be attributed to the change in initial gust strength (caused by the leakage from the slats) throughout the frame. This data confirmed that the effective angle assumption, which was used to determine the 0degree effective angle case, was correct.

Three more pairs of images were used for the comparison of the gust at this power to a steady state wing. They can be seen in Figure 66, Figure 67, and Figure 68, which 
compare $t^{*}=6.91, t^{*}=10.71$, and $t^{*}=26.79$ to wings at comparable effective angles of attack. The latter two images, Figure 67, and Figure 68, are of practically the same estimated effective angle of attack, but at very different points in the time sequence of the actuation. They do a good job of presenting the vorticity lag effect when compared with the estimated angle of attack. Note that all static wing cases were rotated to the 0degree reference frame for ease of comparison. When comparing all three selected times to a wing at similar static AoA there was hardly any visible similarities. The size, strength, and path the vorticity follows was different between the gust and wing at static angle cases.

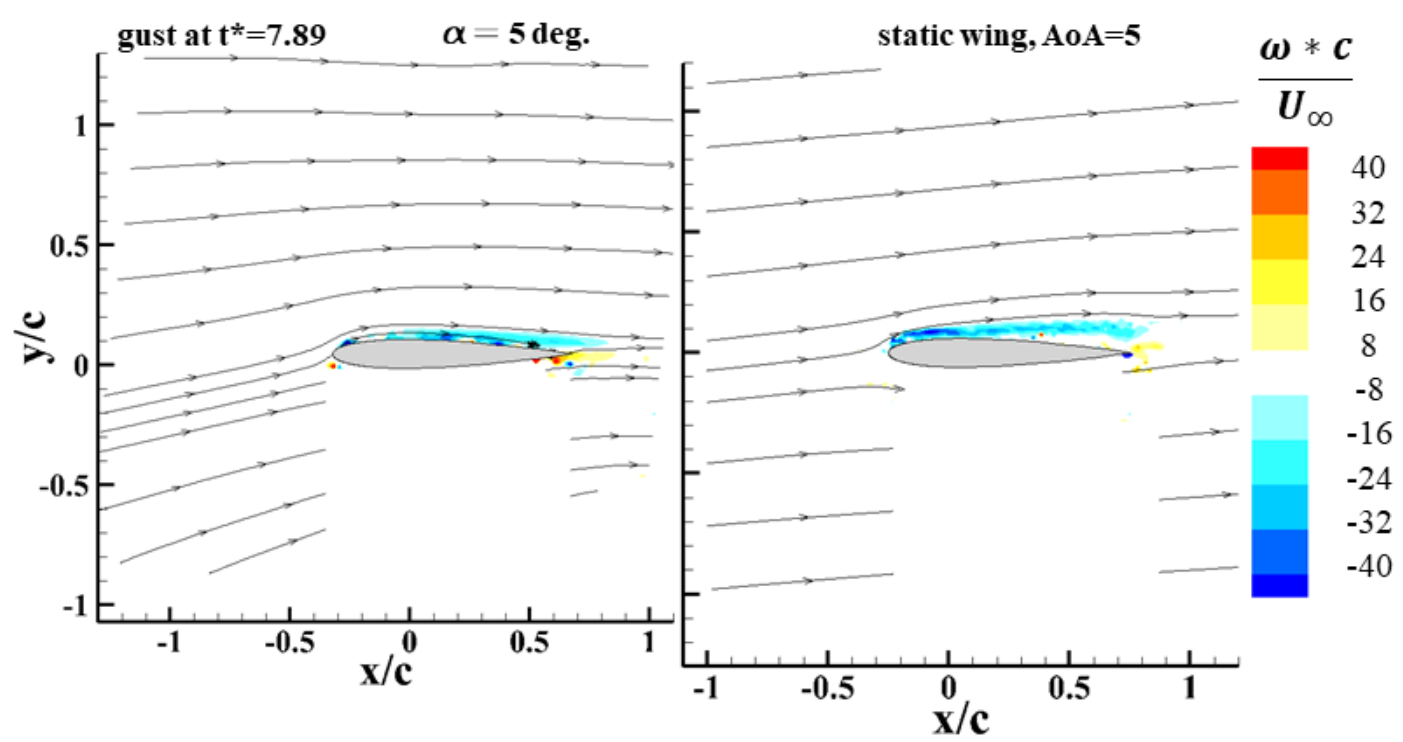

Figure 66: Early full-power-gust/static wing comparison; 5-degree effective angle. 


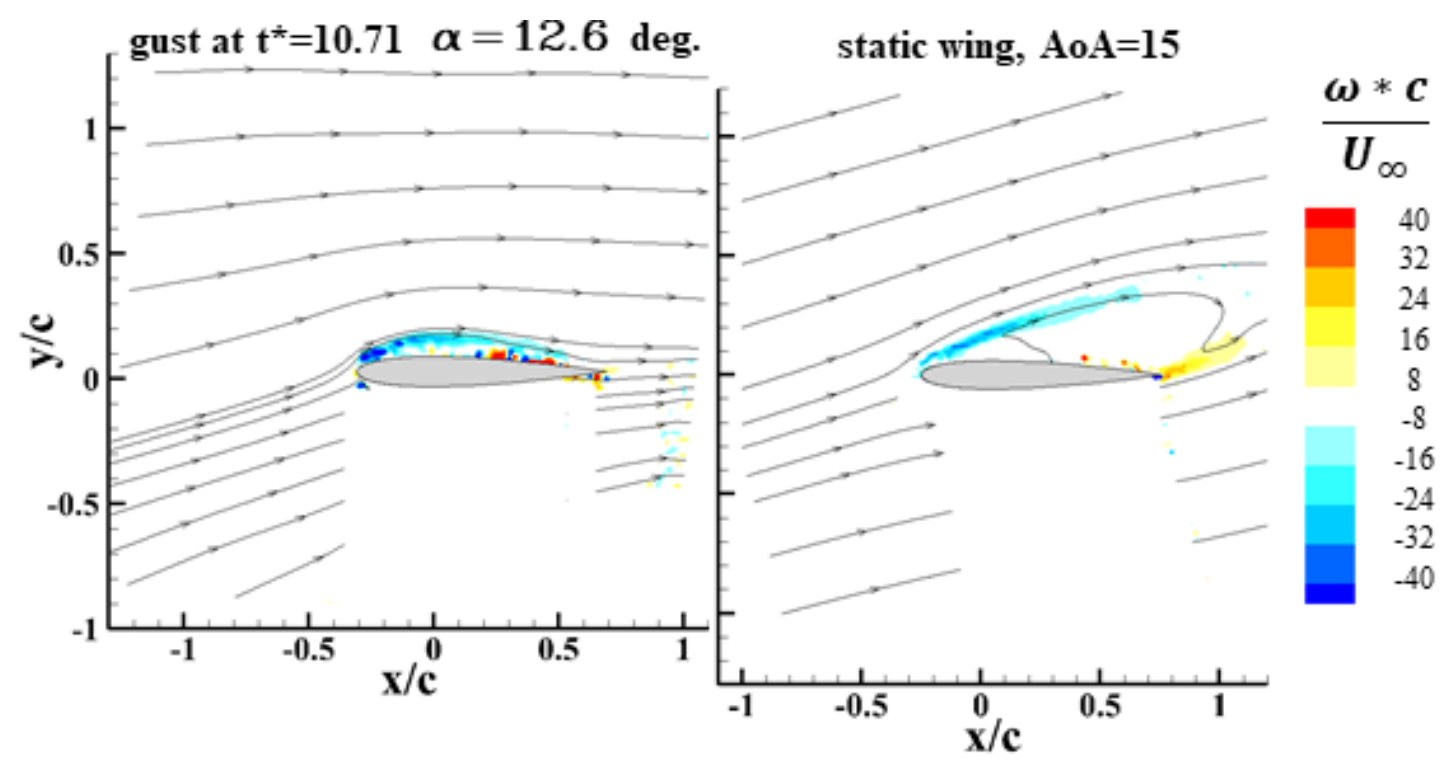

Figure 67: Mid full-power-gust/static wing comparison; 13 and 15-degree effective angles.

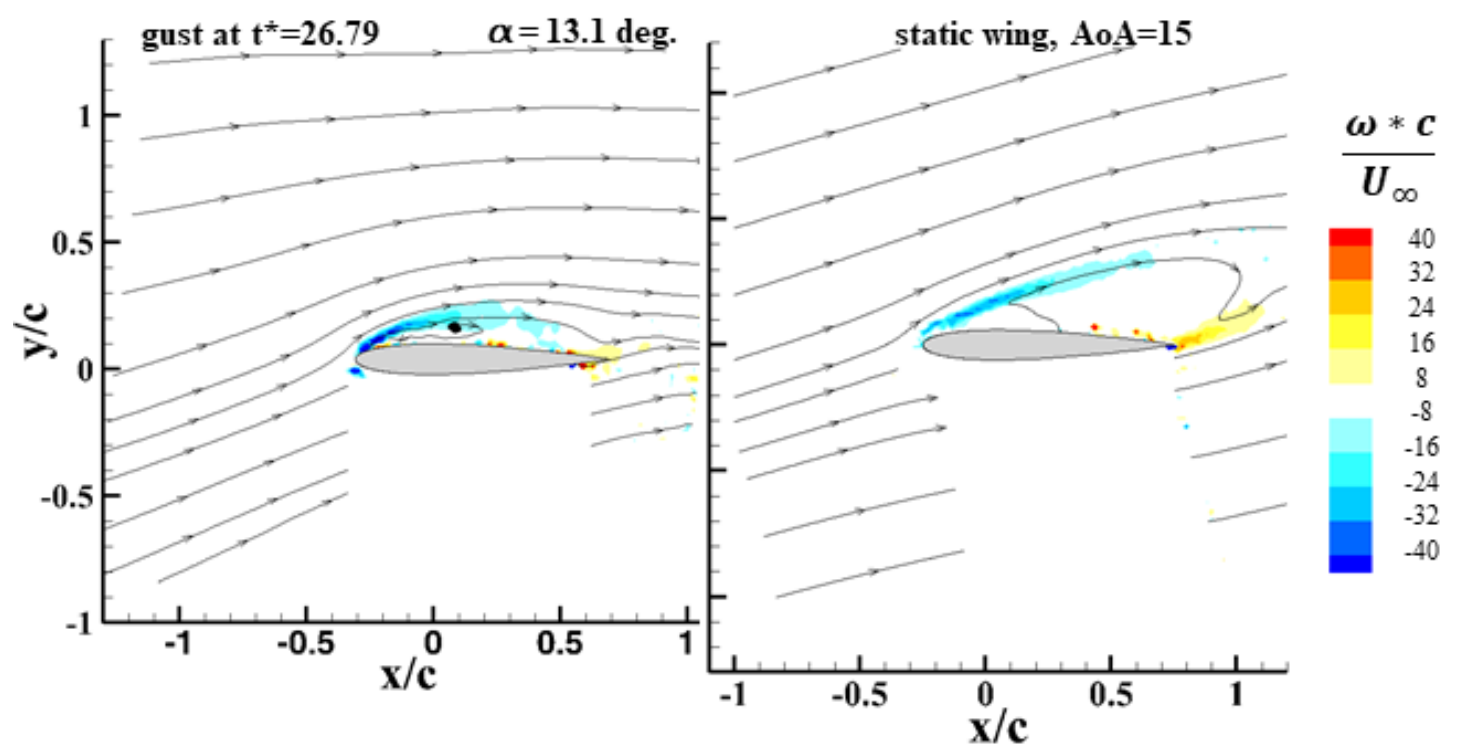

Figure 68: Late full-power-gust/static wing comparison; 13 and 15-degree effective angles.

Based on these comparisons there may be some possible explanations as to why the flows appear so dissimilar. First, the estimation of effective angle may not be an accurate method for estimating the flow angle observed by the wing. A more likely explanation for this difference is that the transient behavior of the gust cannot be replicated as a series of static flow conditions. Some guess as to which explanation is 
correct can be made by looking at the streamlines. The streamlines compare far better to one another than the vorticity plots would suggest. From this, it seems to indicate that the second hypothesis is more accurate. This result calls into question many of the gust modelling methods which use quasi-static flow assumptions.

While it would be possible to select gust frames and static data that looked more similar, this comparison would be entirely arbitrary and of no particular value in understanding gust-wing interactions. Furthermore, these comparisons would appear similar in terms of vorticity strength, but the path the vorticity would follow would still not be similar, see Figure 69 . None of the steady wing cases had the curvature in vorticity path shown in the gust interaction. This means that even if steady wing angles were chosen to create flows that better match the gust-induced separation, the path the vorticity followed would still not match. Furthermore, for all of the compared steadystate angle of attack cases there was more positive vorticity shed at the trailing edge than for the gust cases. Overall, this suggests that for any significant gust encounter, the gust-wing interaction cannot be modeled as a series of steady wings at set angles of attack.

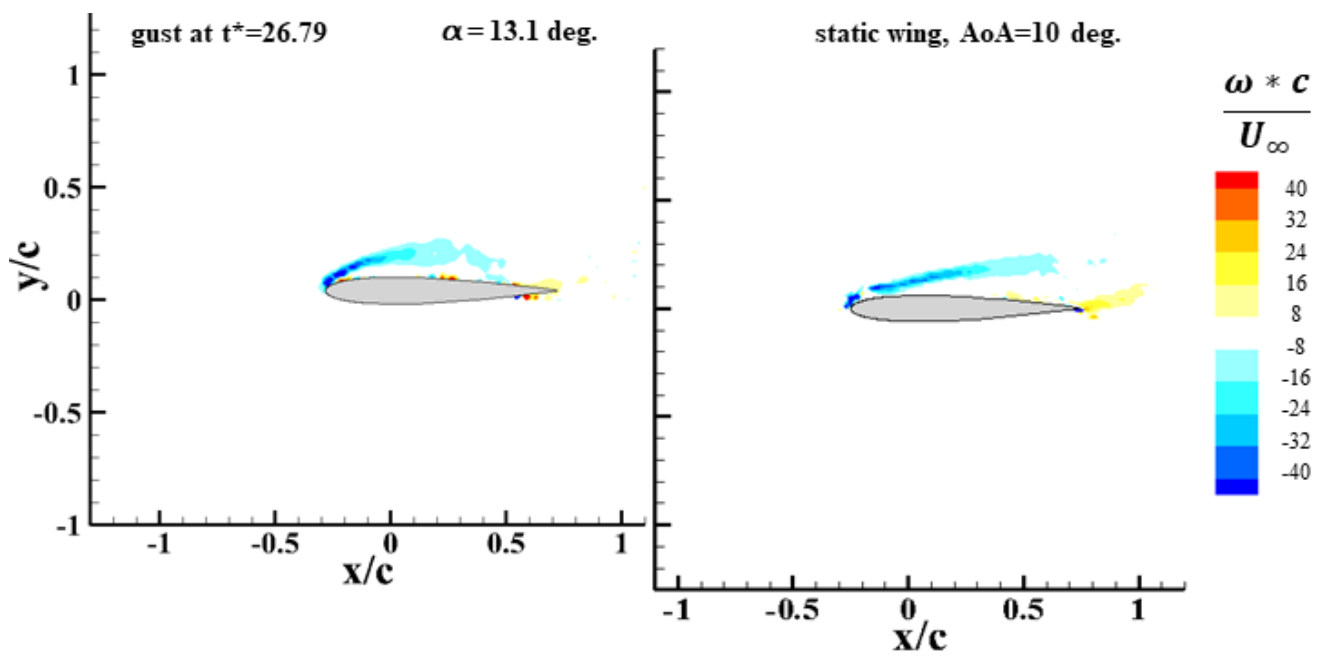

Figure 69: Like-flow comparison 


\subsubsection{Power 5 and 10-degree Effective AoA cases with Static Wing Comparison}

In addition to the neutral condition, effective angles of both 5 and 10 degrees were tested. These angles were selected to analyze other key flight condition points of MAVs. The 5-degree effective AoA condition is a common cruise condition of fixed wing MAVs, while the 10-degree AoA was expected to begin the interaction in a statically stalled condition. It was thought that there would be a more significant stall event for a wing that entered a gust while already at positive angles of attack. This hypothesis held true and can be seen in the 5-degree initial effective angle of attack case shown in Figure 70.

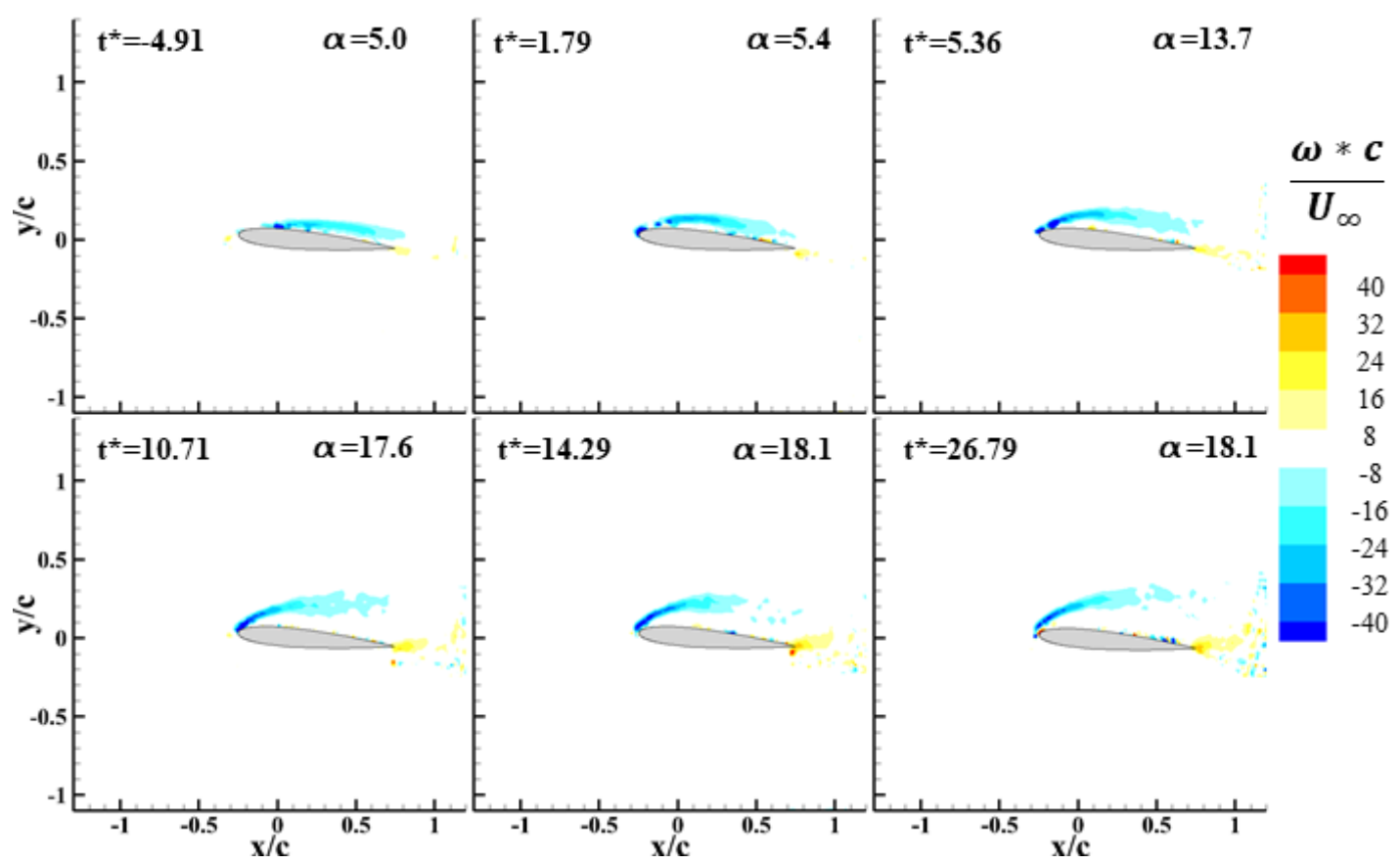

Figure 70: Actuating full power gust; wing at 5-degree initial effective angle.

As in the 0-degree case, the flow curved toward the trailing edge as it separated from the wing. For this initial effective angle, the flow separated earlier, and the wing ended in a deeper stall condition. The effect the gust had on the wing can be seen as 
early as $t^{*}=1.2$ for this case and a steady state was reached by $t^{*}=15.7$. The faster transition to a stalled state was likely due to the wing being closer to its static stall point.

Selected convective times are again shown side by side next to steady state wings at comparable angles of attack with no gust present. These images can be seen in Figure 71 through Figure 74 and are all rotated to a 5-degree reference frame for ease of comparison.

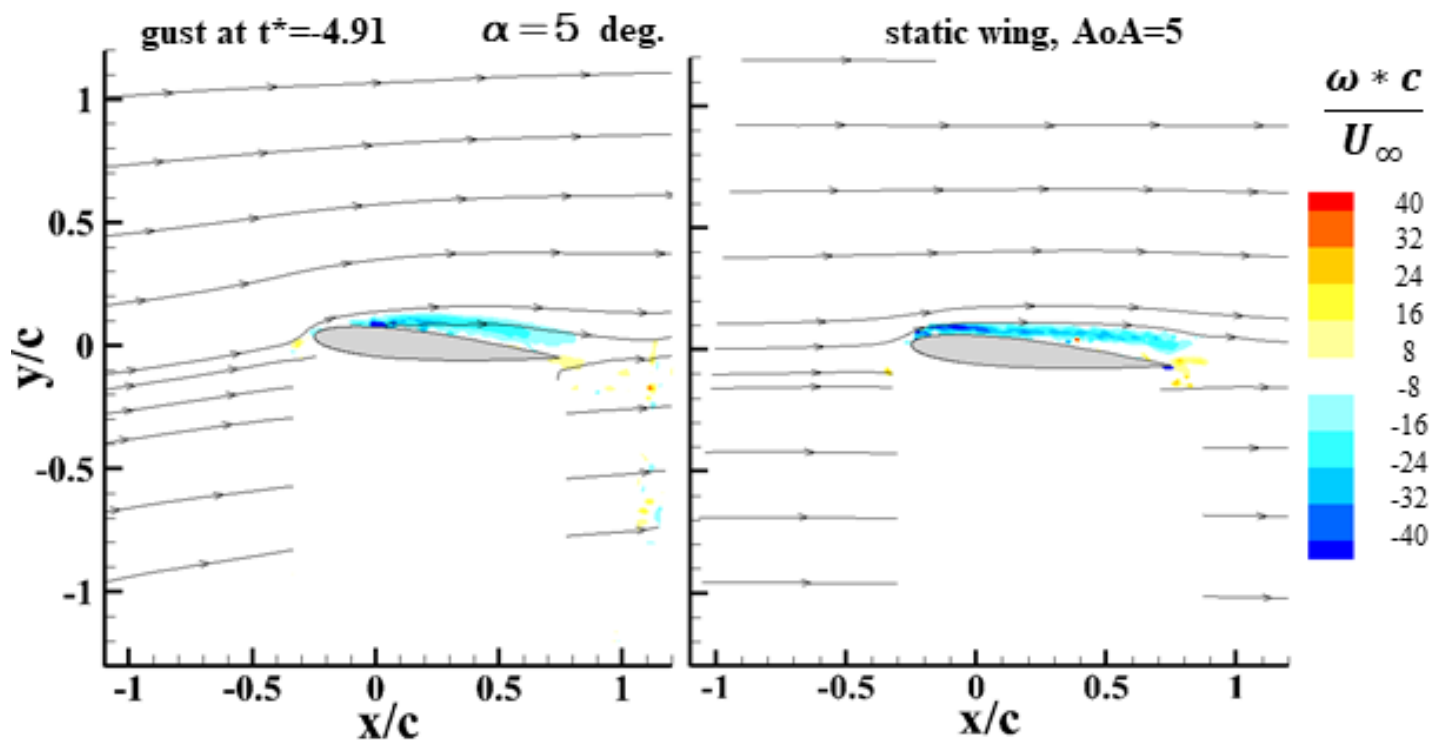

Figure 71: Pre full-power-gust/static wing comparison; 5-degree initial effective angle.
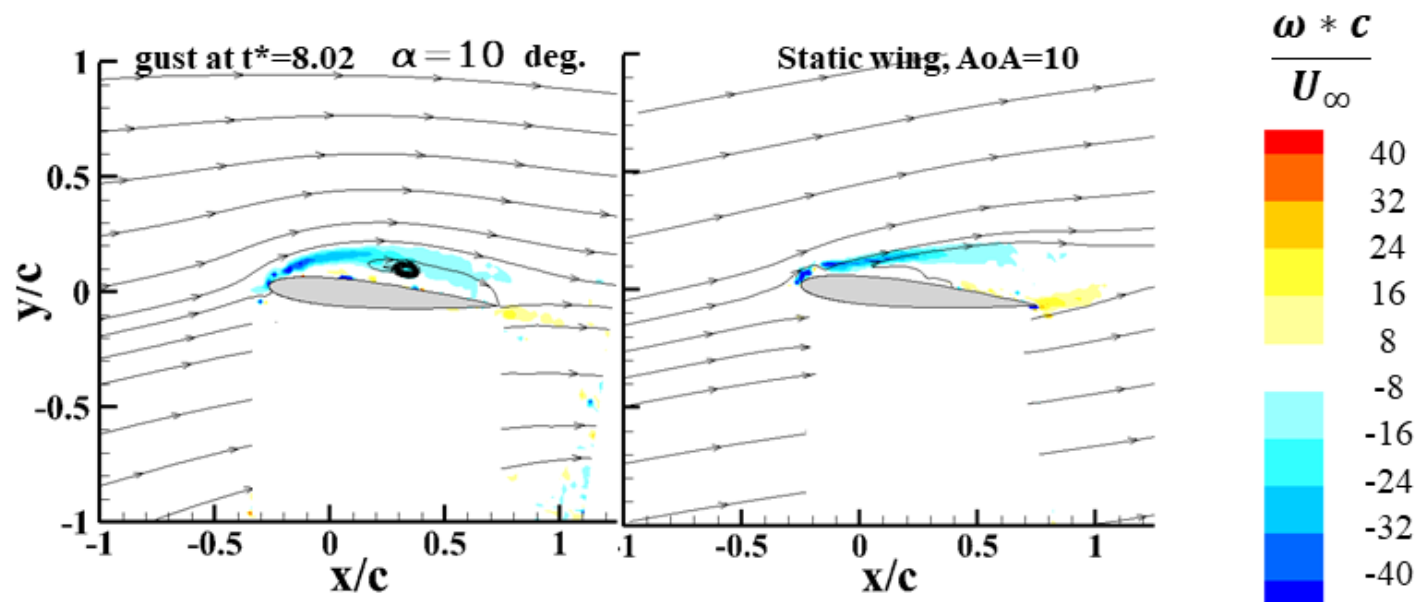

Figure 72: Early full-power-gust/static wing comparison; 10-degree effective angle. 


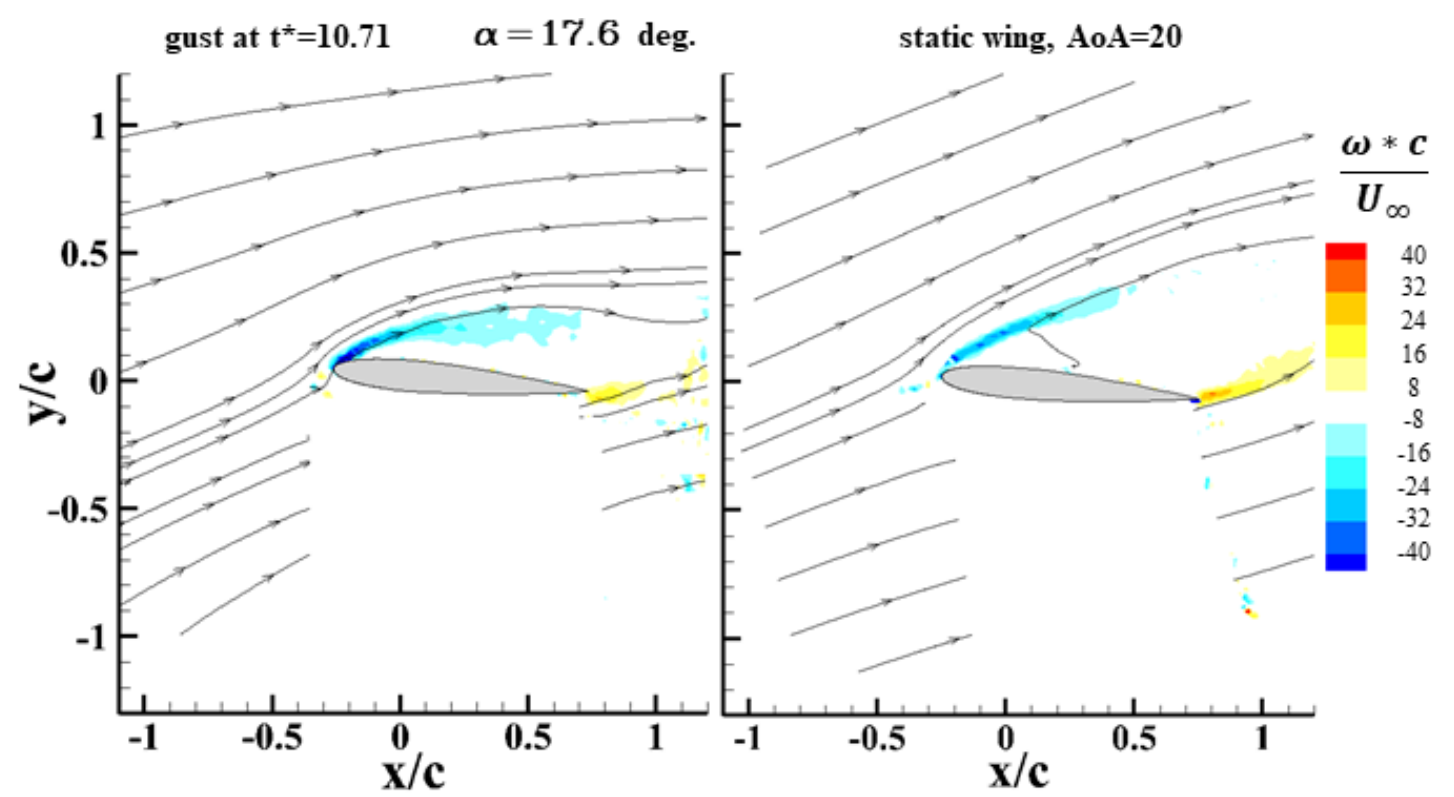

Figure 73: Mid full-power-gust/static wing comparison; 5-degree initial effective angle.

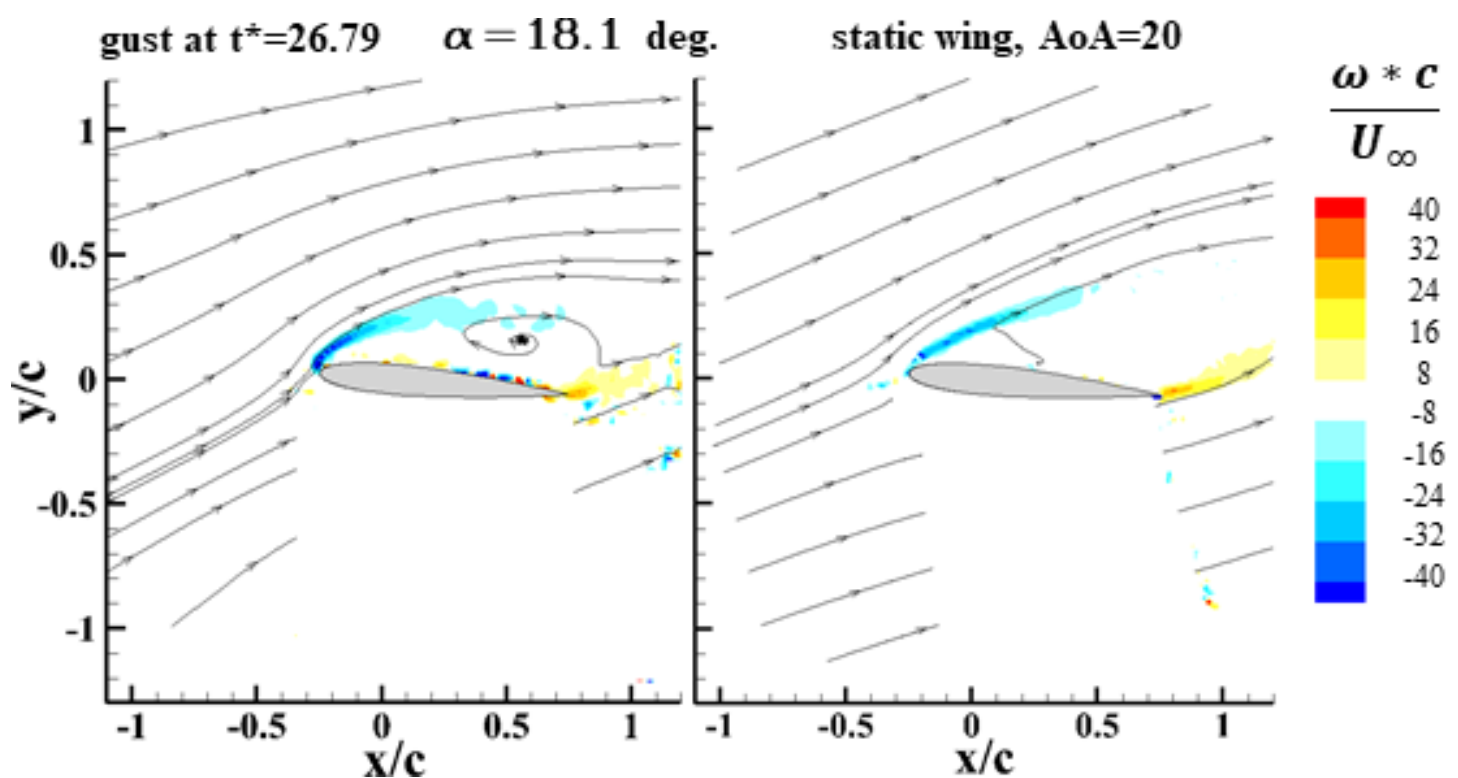

Figure 74: Late full-power-gust/static wing comparison; 5-degree initial effective angle.

Similar to the 0-degree AoA case, there were many similarities between the pregust and static data in Figure 71. The vorticity seen shed from the wing for the gust actuation cases in Figure 72, Figure 73, and Figure 74 match well with the strength of vorticity created by the wing at static angles of attack, but again the vorticity followed a more curved path and stays closer to the top of the wing experiencing the gust. This 
difference; however, becomes more subtle towards the very end of the gust actuation. Still, leading edge shed vorticity diffused more rapidly in the gust compared to the static case. This result further challenges the use of static data for evaluation of unsteady gusts.

The final full-power gust data set collected was with the wing at a 10-degree effective angle of attack before gust actuation. Figure 75 shows selected $t *$ for this gust actuation.

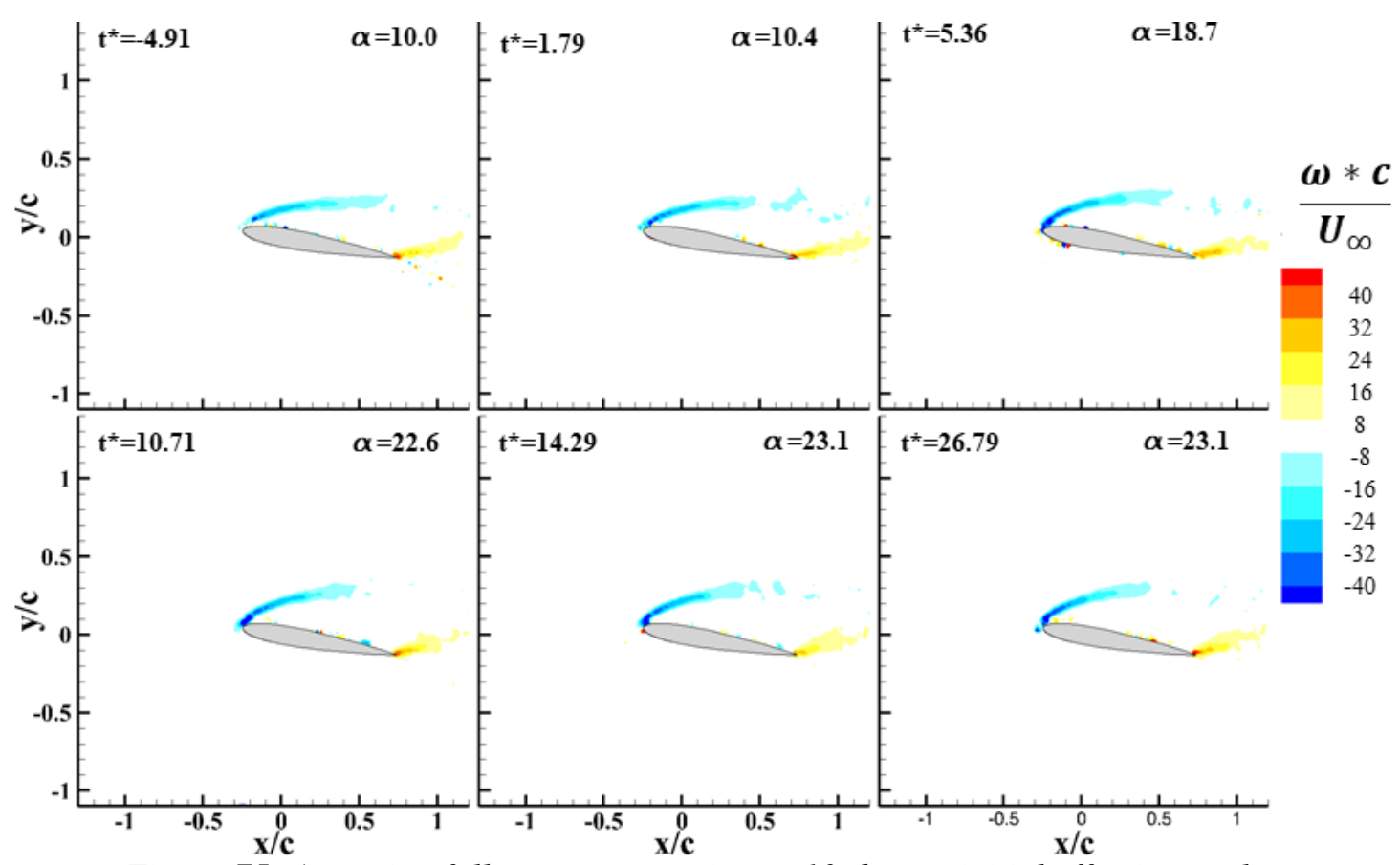

Figure 75: Actuating full power gust; wing at 10-degree initial effective angle.

For this case the wing started the gust interaction in a stalled state. This means that a NACA 0012 airfoil at a $\mathrm{U}_{\infty}$ of $1.5 \mathrm{~m} / \mathrm{s}$ must stall somewhere between 5 and 10 degrees. It is known from other studies in the MAWT facility that the NACA 0012 tested stalls around 8-9 degrees in a clean tunnel configuration, though the gust generator leakage may impact the exact stall angle in this study. Due to the high levels of separation present before the start of gust actuation, the gust has less of an impact 
on the wing. The shedding angle of the leading-edge vorticity increased as the gust was actuated but the wing remained in a stalled state and no coherent vortex structures were observed.

As with other cases, the wing was compared to a steady state wing at positive angle of attack. These results can be seen in Figure 76 through Figure 79. All images are rotated to a 10-degree reference frame for ease of comparison.

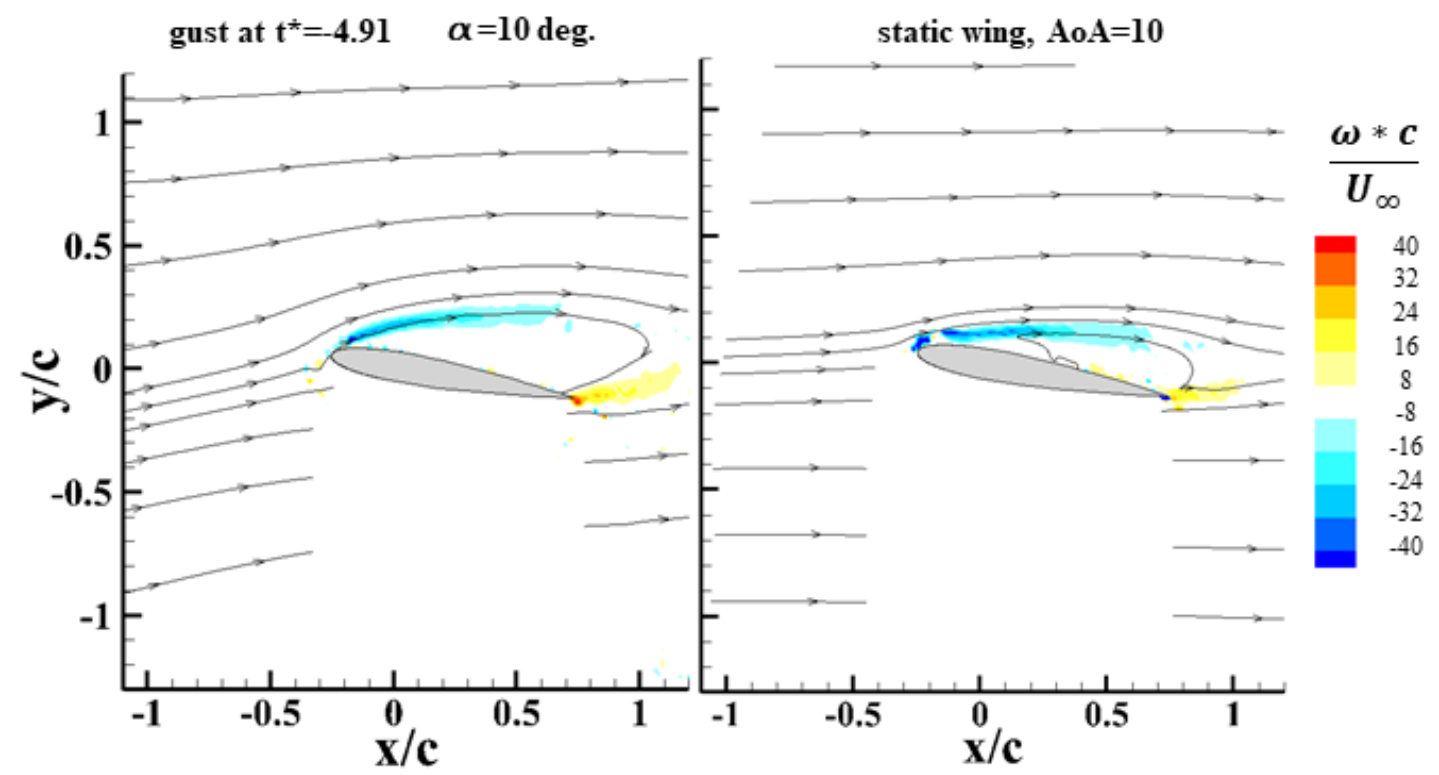

Figure 76: Pre full-power-gust/static wing comparison; 10-degree effective angle.

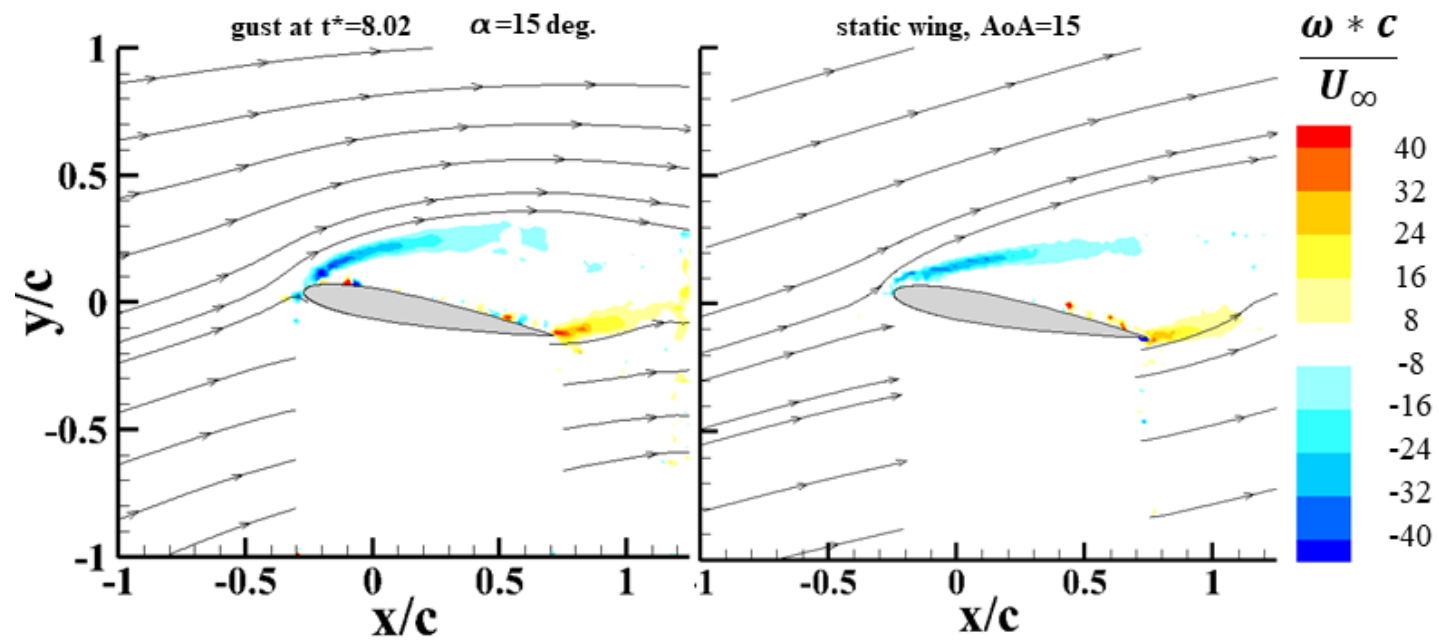

Figure 77: Early full-power-gust/static wing comparison; 15-degree effective angle. 


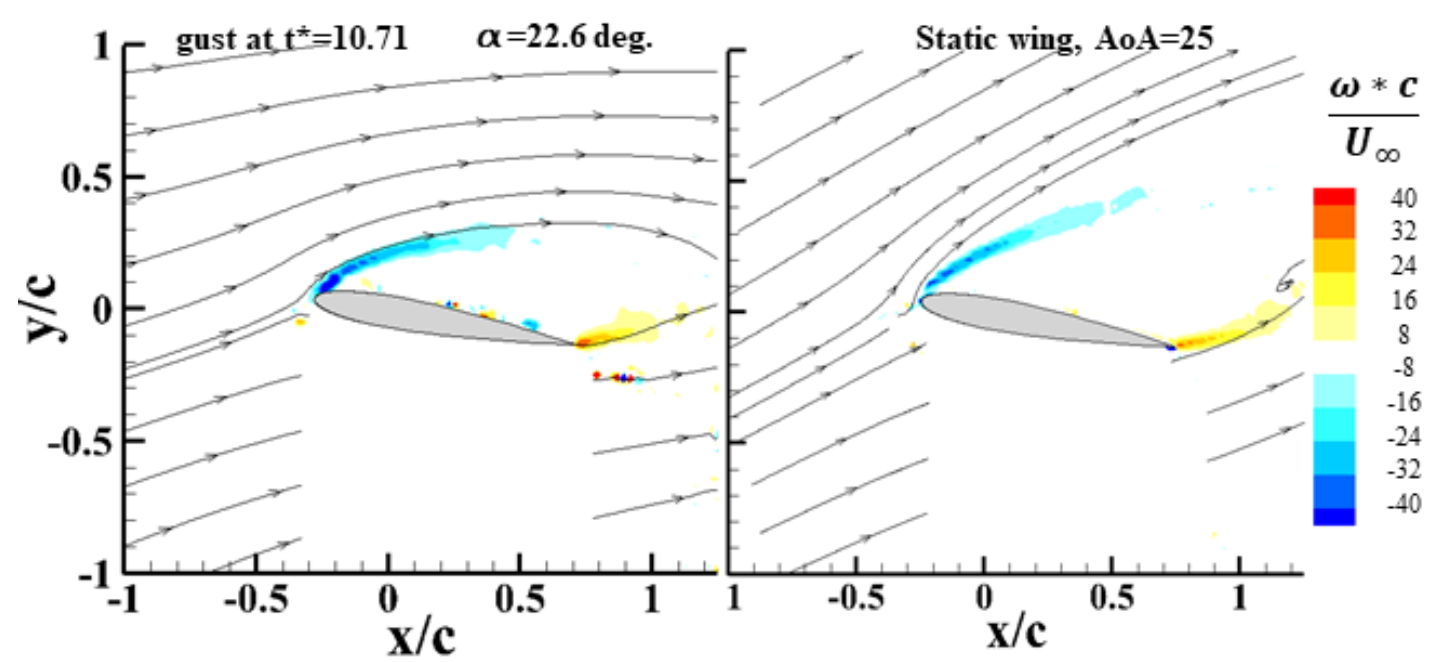

Figure 78: Mid full-power-gust/static wing comparison; 10-degree initial effective angle.

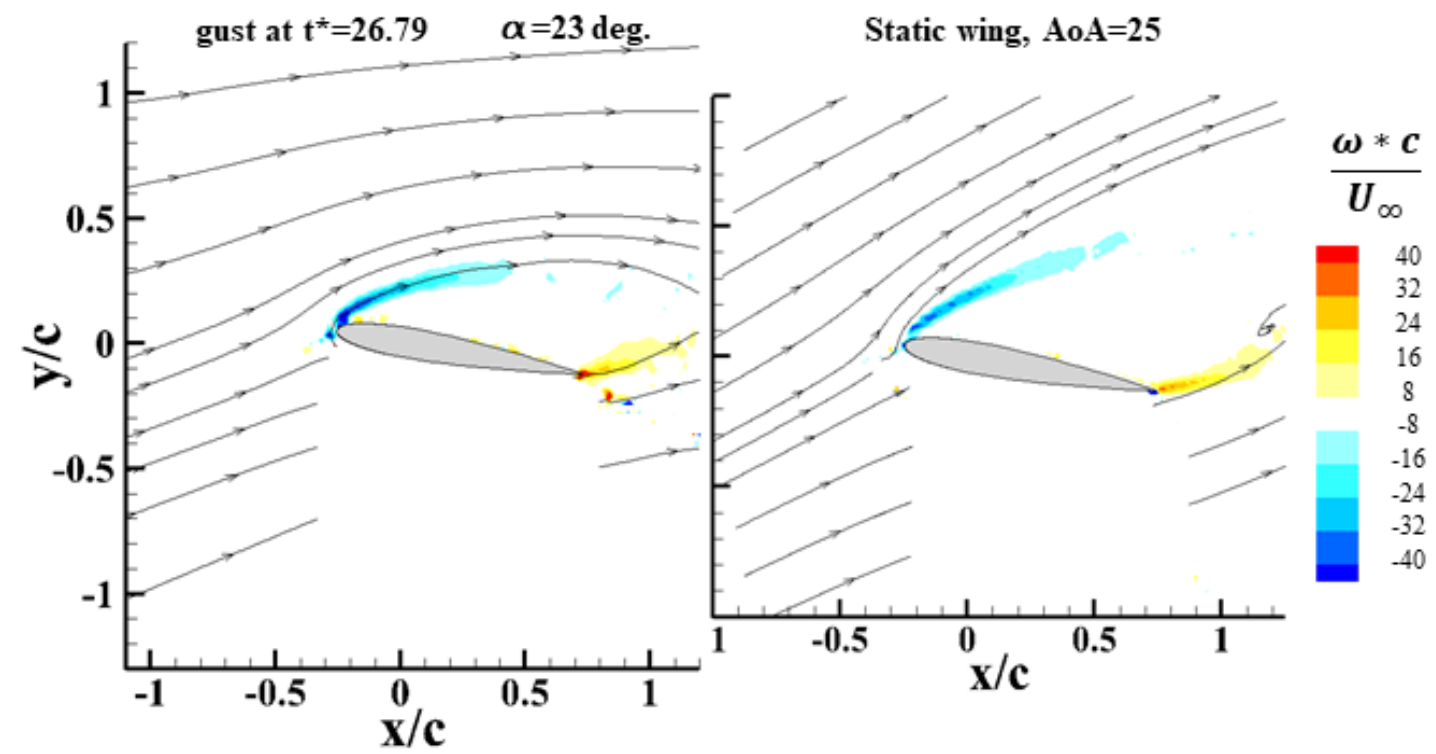

Figure 79: Late full-power-gust/static wing comparison; 0-degree initial effective angle.

Unlike prior cases, the effective angle correction at the 10-degree case was not as effective as the non-stalled cases. The flow was much further separated in the angle corrected gust case than the steady case. While the effective angle corrections were effective pre-stall, this was not the case post-stall. It is likely that the pre-gust actuation vertical velocity present combined with the stall behavior of the wing to create a larger separated region when compared to the static case. 
Oddly, the remaining t* to static angle comparisons matched far better than when the wing began the interaction at lower angles. When starting in a post-stall condition, the flow behavior was dominated by the stall condition and less impacted by the gust. The vorticity did not bend toward the trailing edge as much in the 10-degree case compared to lower starting angles, resulting in better correlation to static angles. It may be possible that for wings with initially high geometric angles of attack, the gust interactions could be modeled as simple increases in angle of attack of a static wing. Further tests would need to be run to confirm this idea, but the concept is limited in its use as few aircraft fly in stalled conditions.

\subsubsection{Mid Power Gust-Wing Interaction}

Since other gust strengths were also being considered, the same recording of the gust actuation process as it interacts with a wing is shown in this section for the mid power gust case. All three starting effective angles of attack were considered again for this power. As before, the starting angle at which the wing needed to be pitched to create the desired effective angle of attack for testing was determined by the pre-gust flow angle found in section 5.1.2. The results and comparison for the 0-degree mid power tests can be seen in Figure 80 through Figure 83. The images have been rotated to a 0-degree reference frame for ease of comparison. 


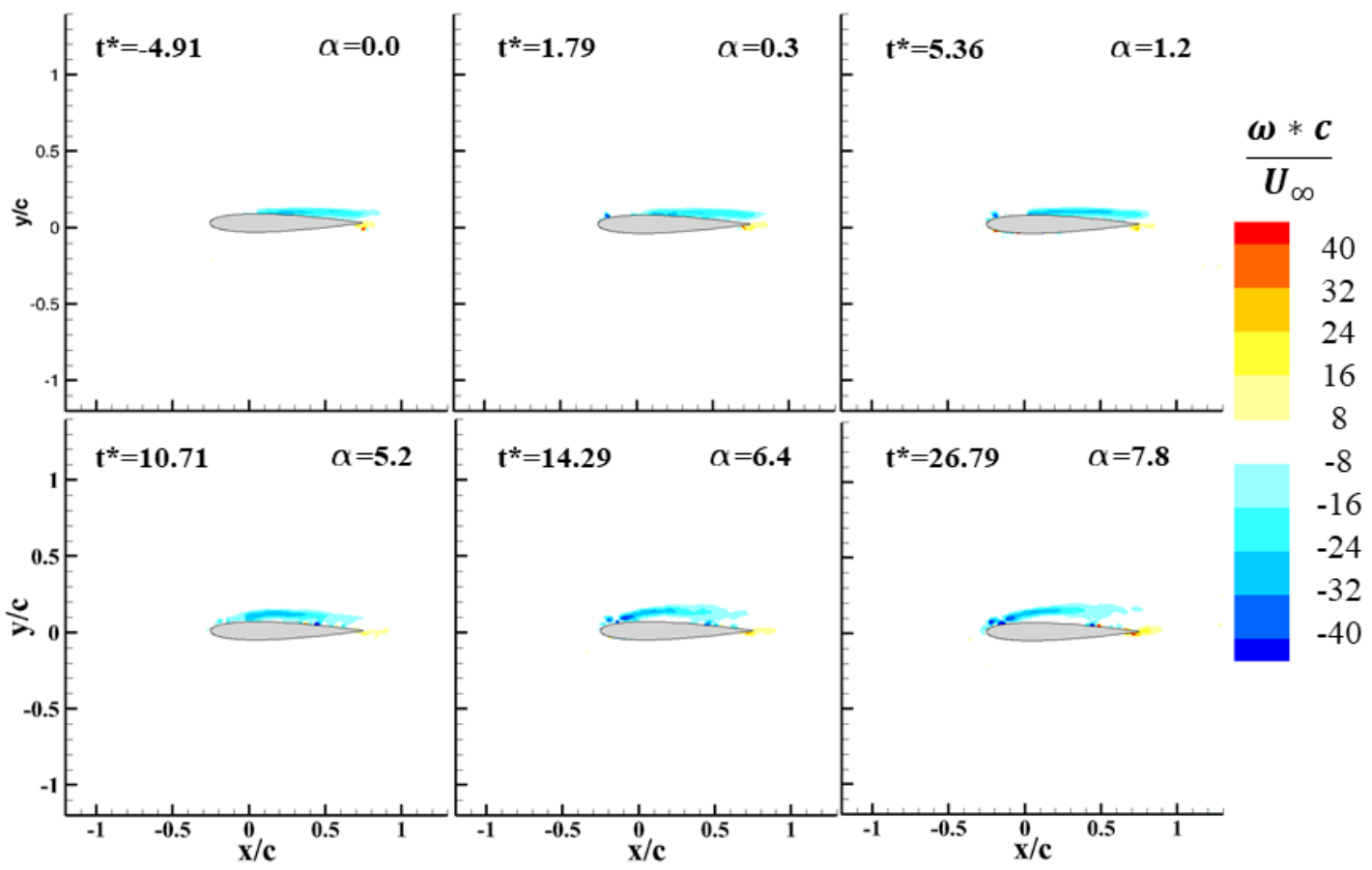

Figure 80: Actuating mid power gust; wing at 0-degree initial effective angle.

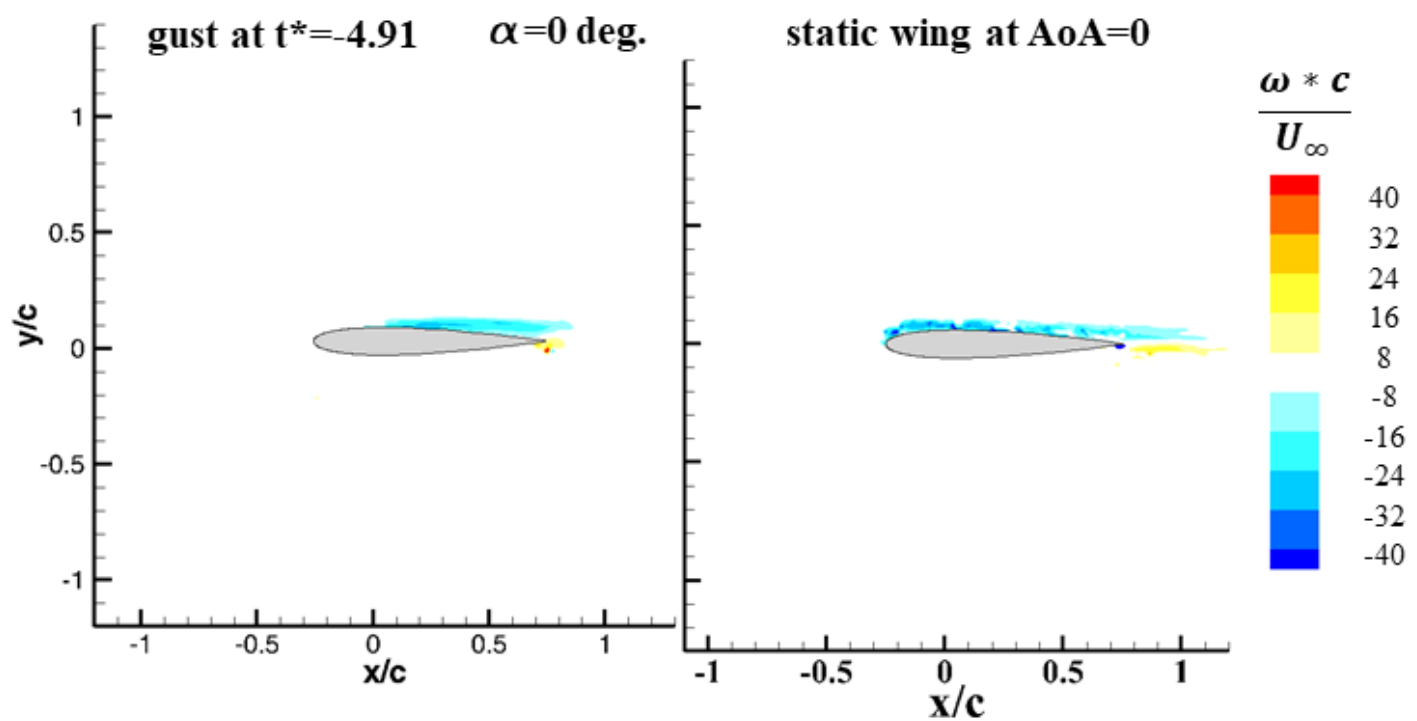

Figure 81: Pre mid-power-gust/static wing comparison; 0-degree initial effective angle. 


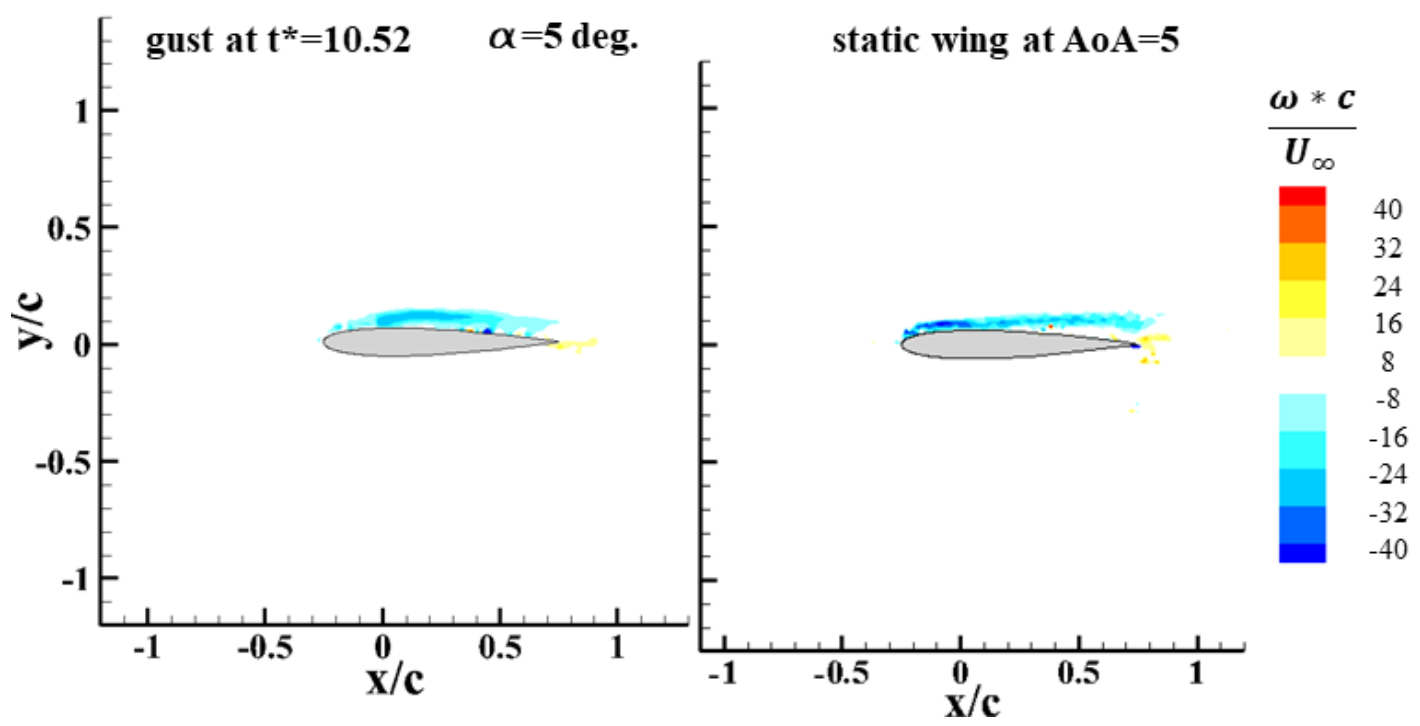

Figure 82 Mid mid-power-gust/static wing comparison; 0-degree initial effective angle.

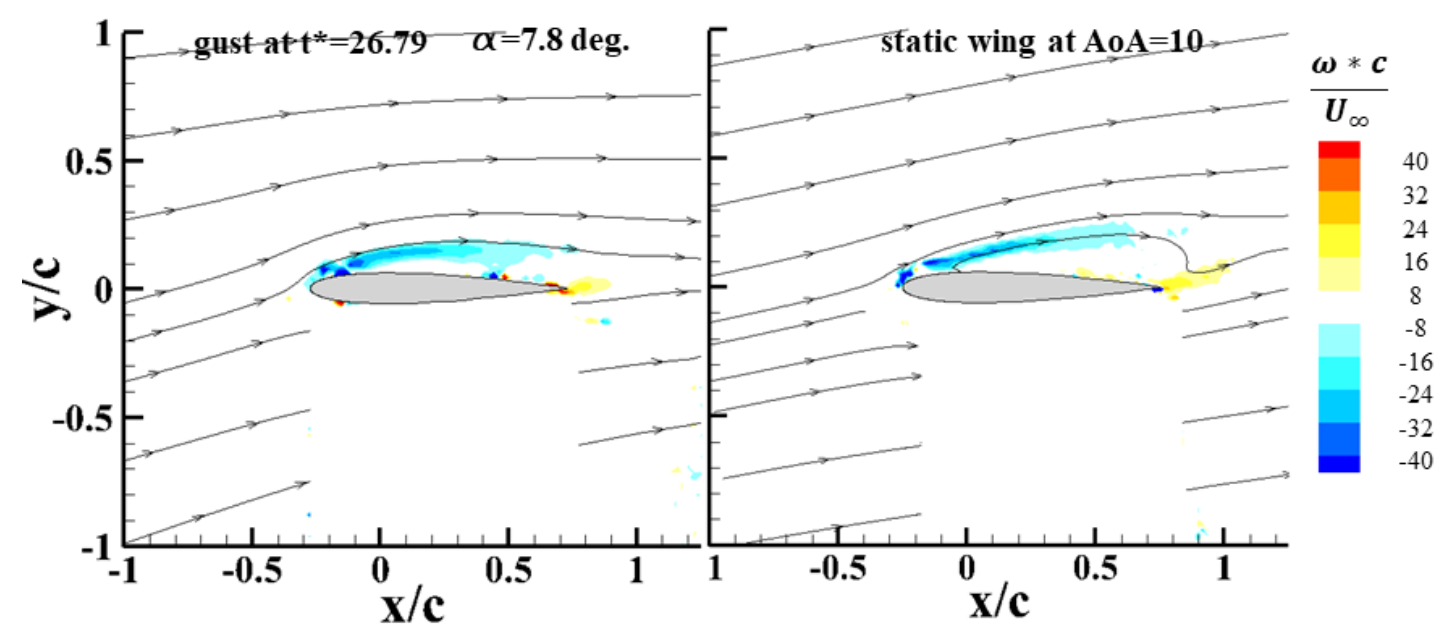

Figure 83: Late mid-power-gust/static wing comparison; 0-degree initial effective angle.

The mid powered gust had less of an effect on the wing than the full powered gust case did. The change in vorticity about the wing is not as large throughout the gust actuation process. The 0-degree angle case for the steady state wing and the pre-gust case show good comparison. The 5-degree stationary wing case compares well to the wing at a 5-degree effective angle due to the gust interaction shown in Figure 82. The vorticity layer was thicker in the gust case and longer in the static wing case. The 10degree static wing in the final comparison is known to be post stall, while the 8.35 
effective angle of attack is right in the middle of the transition region from attached to separated flow. Thus, this comparison is somewhat unfair to make. Still, this comparison shows that the strength of the vorticity between the gust and steady wing cases were similar, but the path the vorticity followed is clearly more attached in the gust case and maintains the arced profile seen previously, while the steady wing is past stall. At lower gust strengths, the pitched wing case is a better approximation. This is expected since proportionally, more vorticity is generated from the mean freestream flow on the wing and less from the gust.

The 5 and 10-degree initial effective angle cases are presented in the appendix as there was a discrepancy with the effective angle correction presented for those cases.

\subsubsection{Low Power Gust-Wing Interaction}

The final gust actuation comparison was done for the low power gust being actuated on the wing. As was predicted by the initial characterization, there was only expected to be a maximum flow angle change of 5.3 degrees. Pairing this low effective angle change and the fact that thus far, the gust-induced effective angle change has not seemed to have as large of an impact on the vorticity as a static change in angle of attack, explains why there is practically no change in the vorticity due to the gust. Due to this low effective angle change and the fact that static wing profiles were recorded at 5-degree increments, only comparisons at effective angles of 0 and 5 were made. The images for this case can be seen in Figure 84 through Figure 92. 


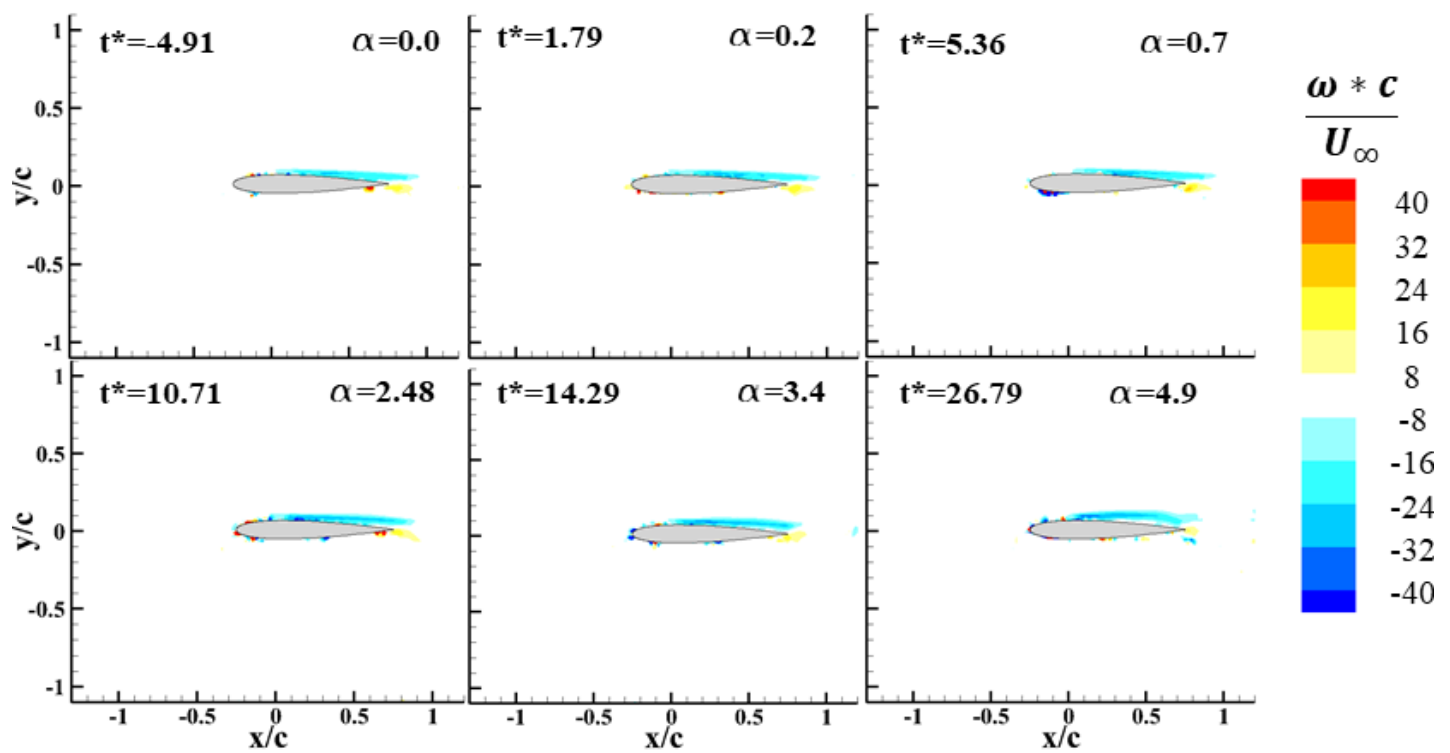

Figure 84: Actuating low power gust; wing at 0-degree initial effective angle.

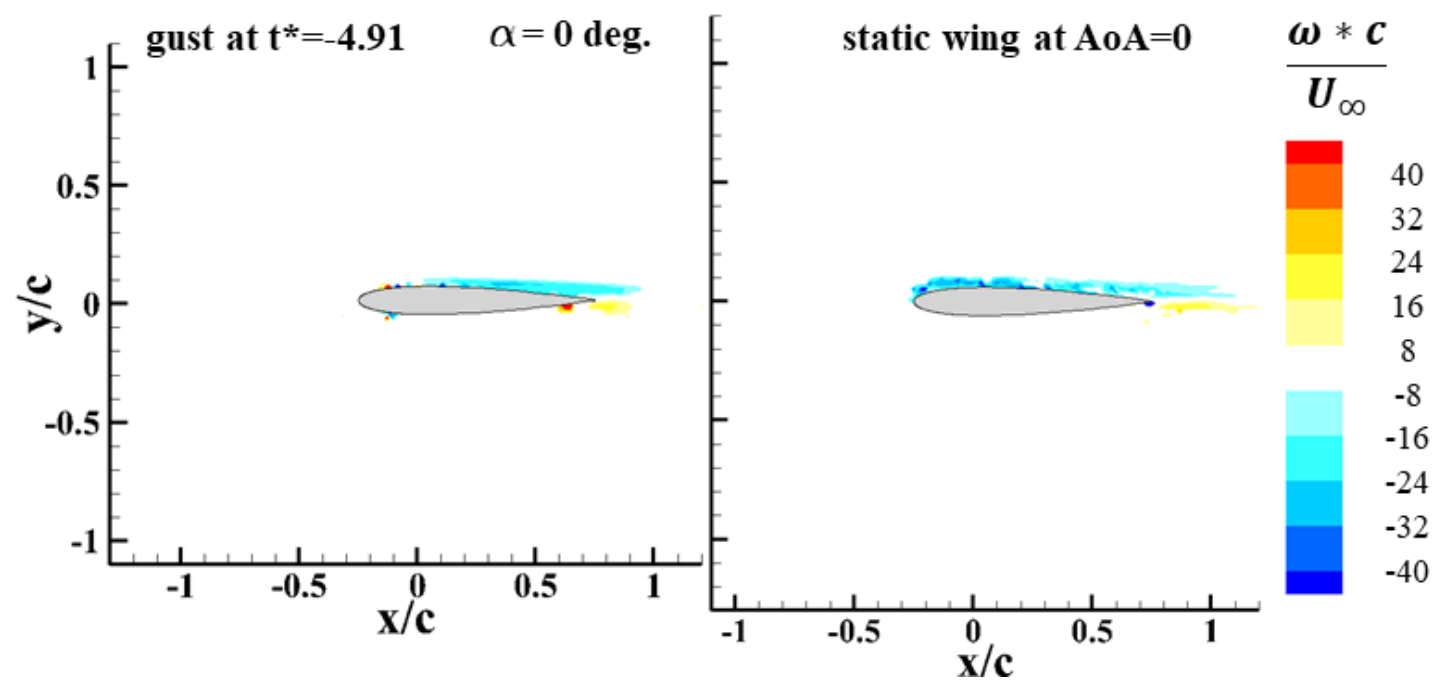

Figure 85: Early low-power-gust/static wing comparison; 0-degree initial effective angle. 


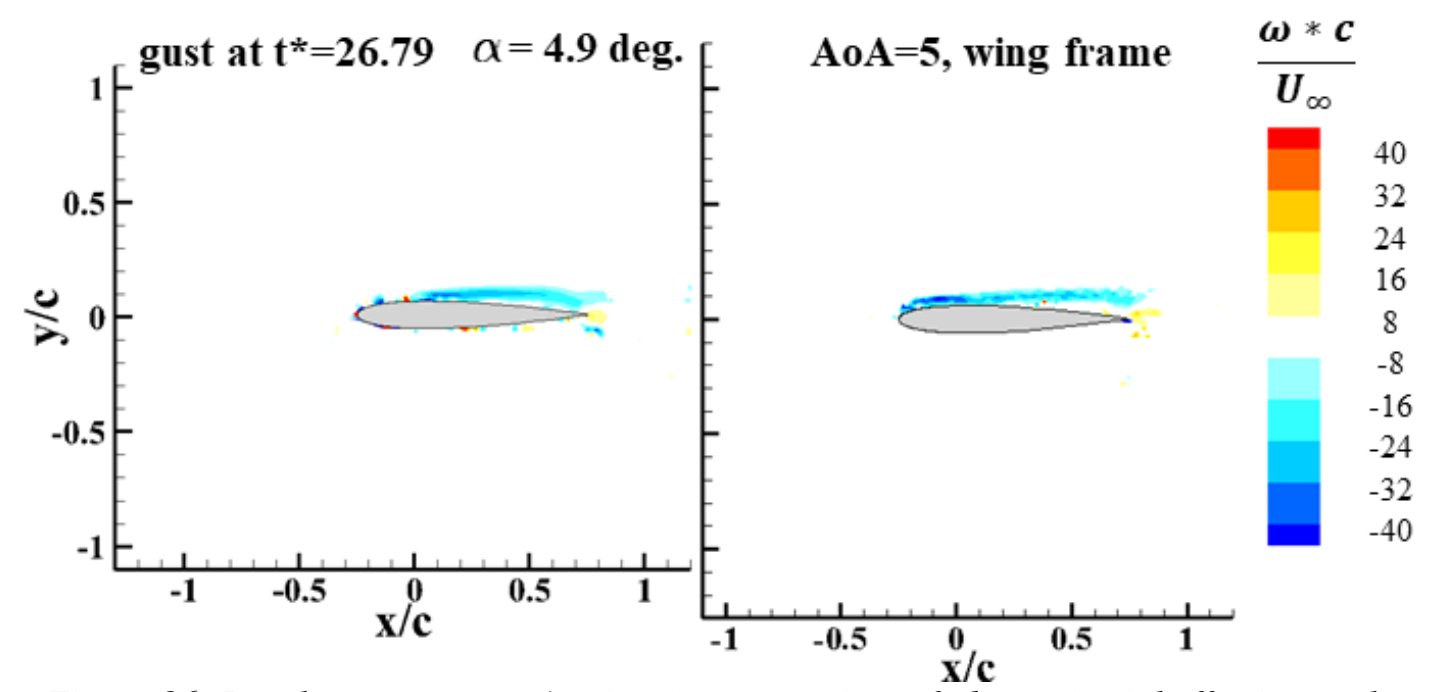

Figure 86: Late low-power-gust/static wing comparison; 0-degree initial effective angle.

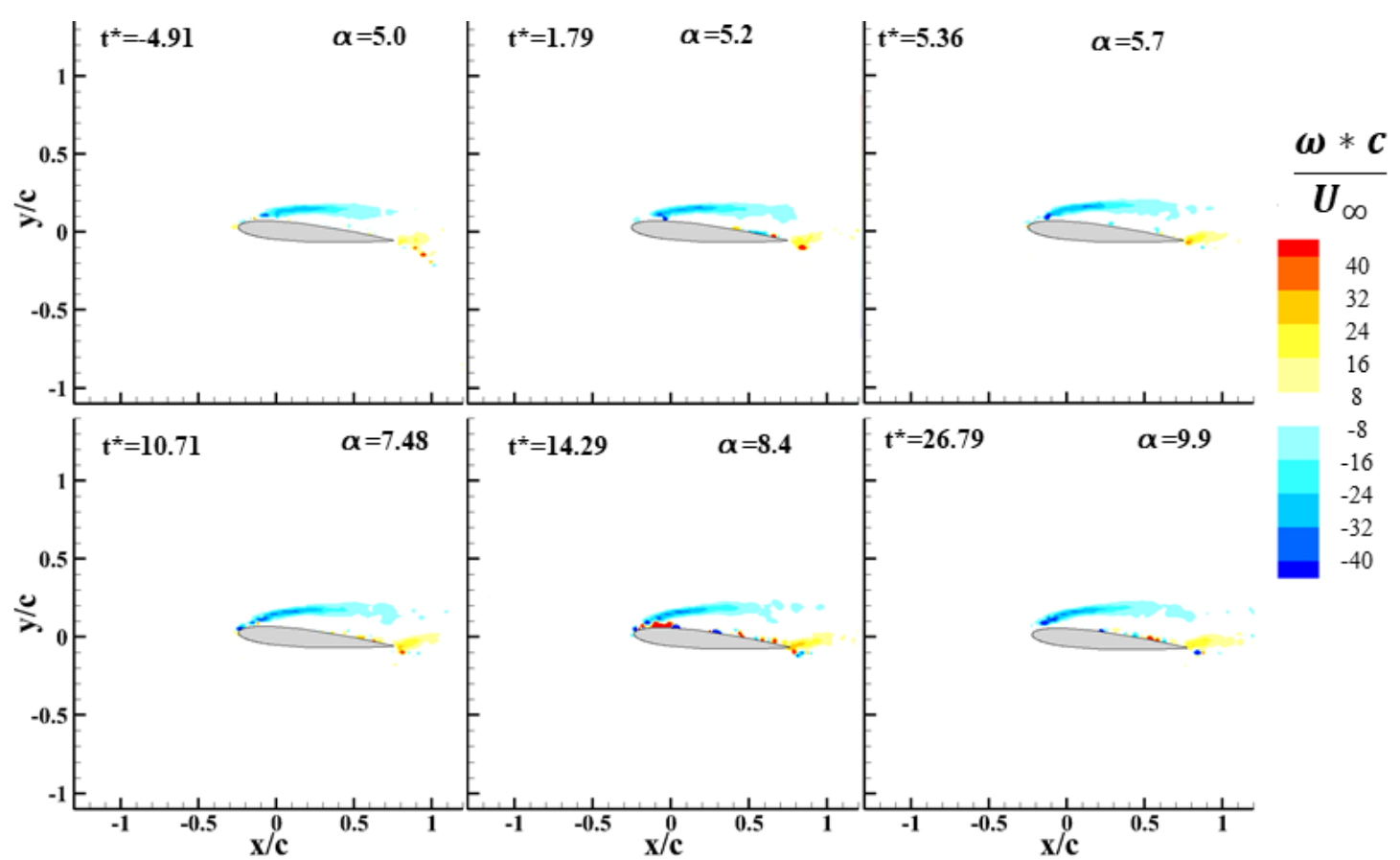

Figure 87: Actuating low power gust; wing at 5-degree initial effective angle. 


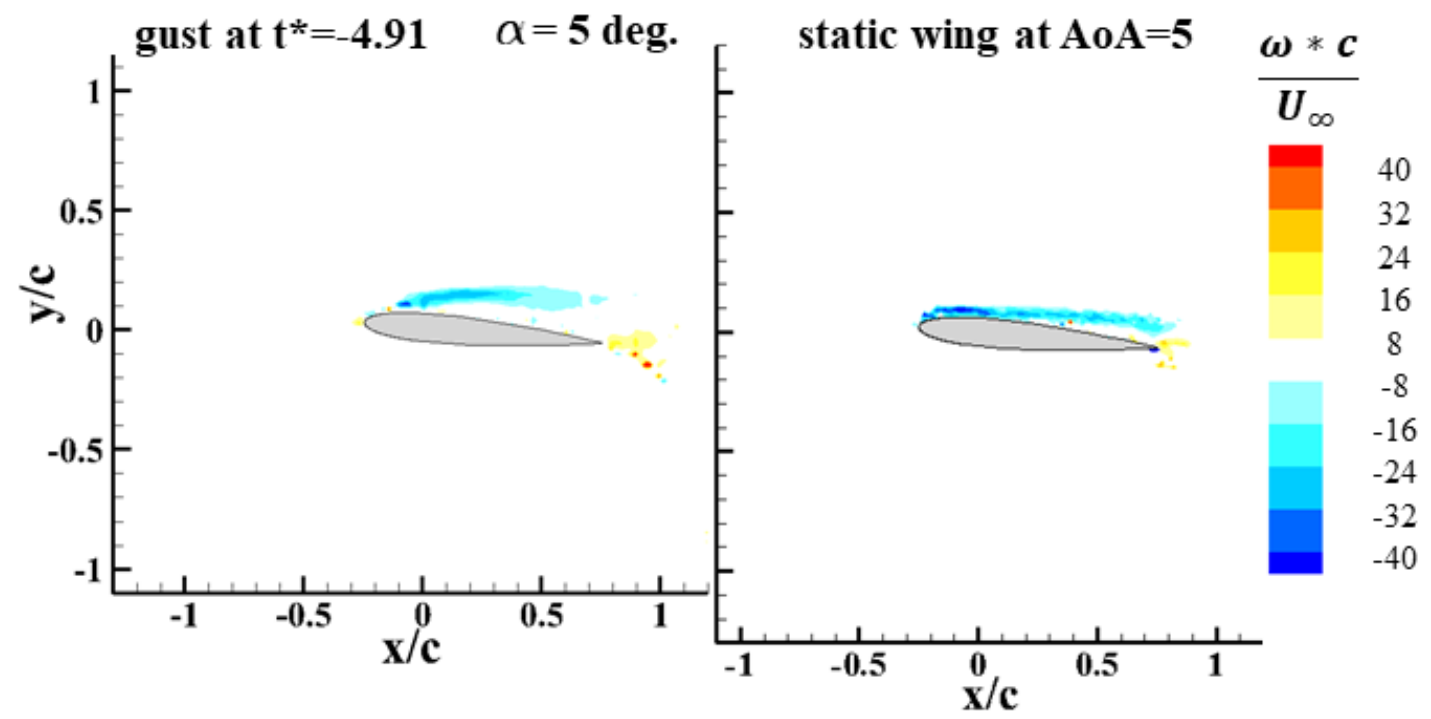

Figure 88: Early low-power-gust/static wing comparison; 5-degree initial effective angle.

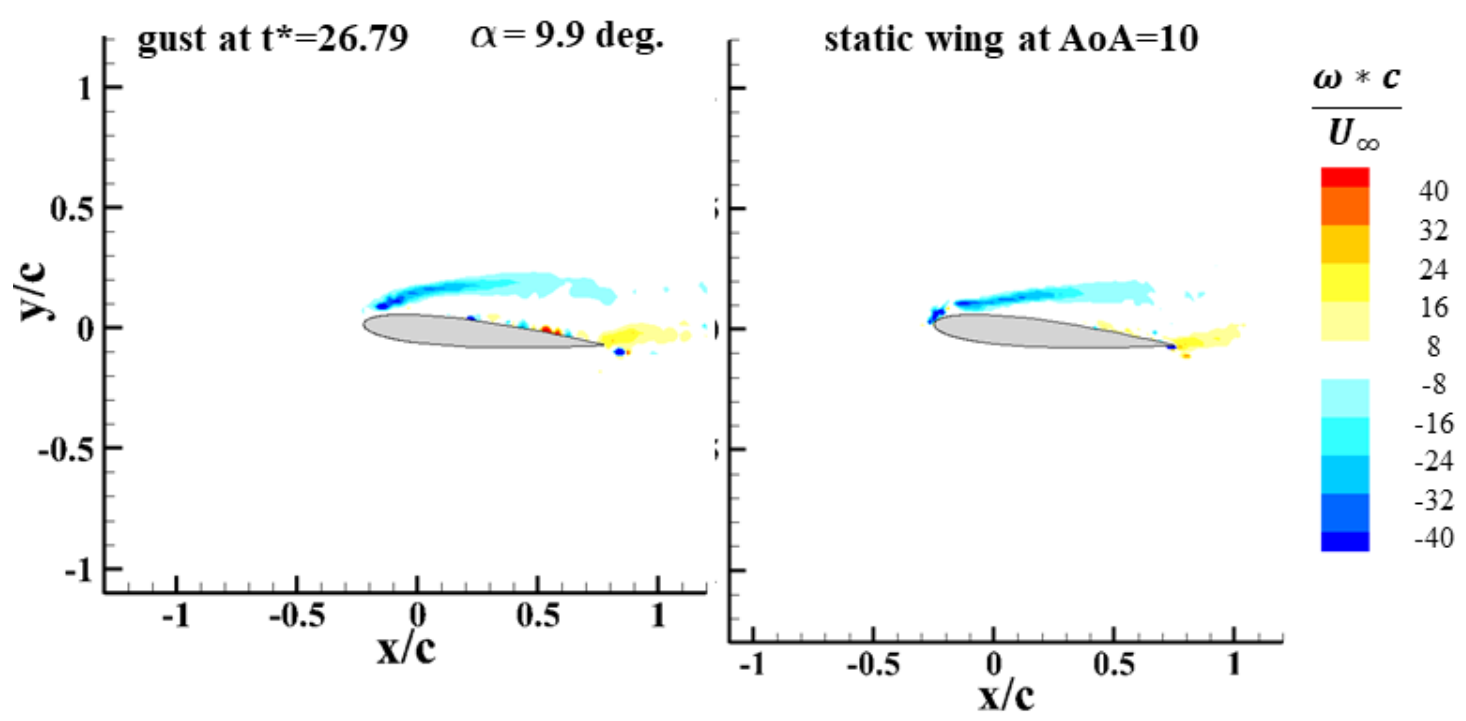

Figure 89: Late full-power-gust/static wing comparison; 5-degree initial effective angle. 


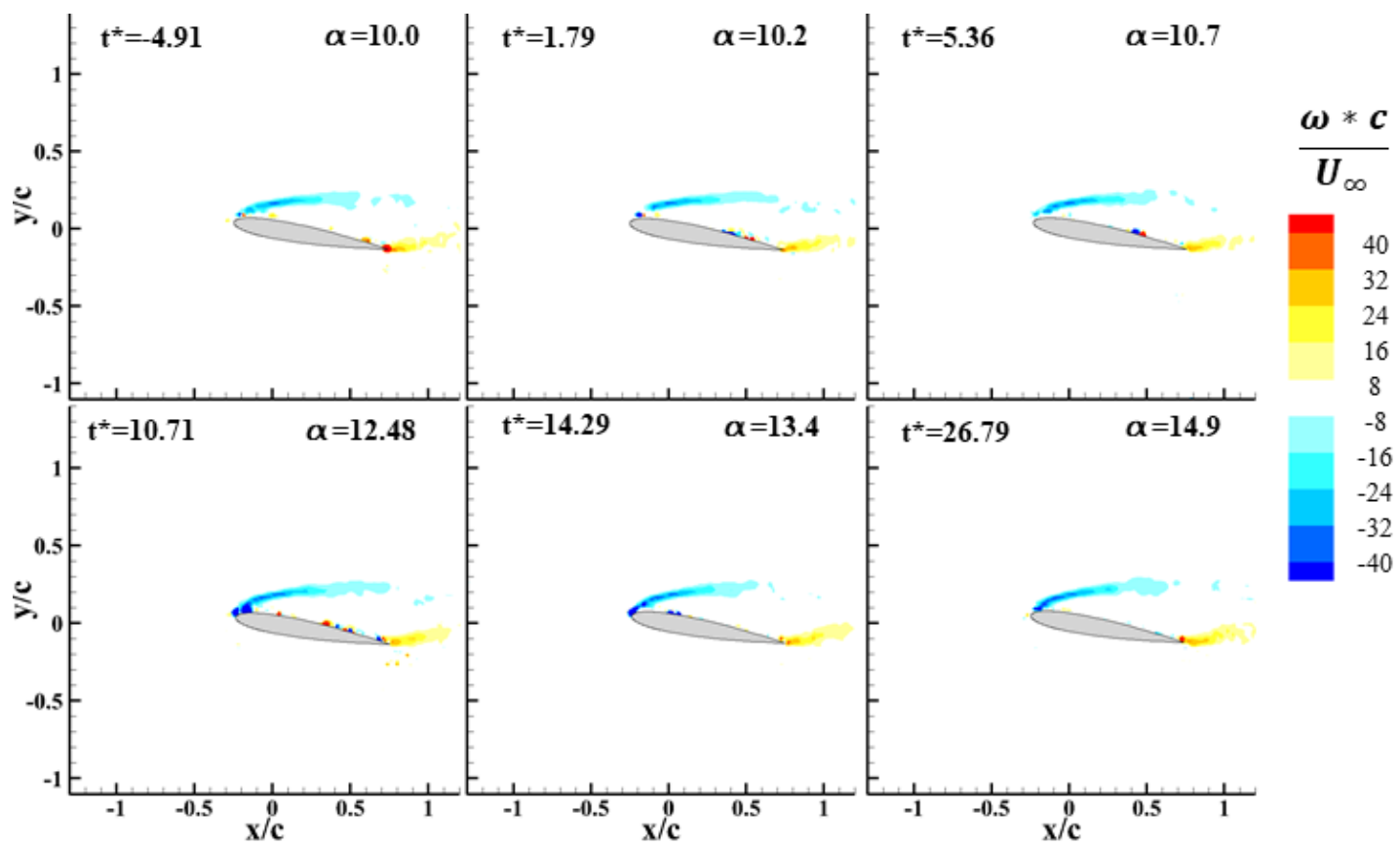

Figure 90: Actuating low power gust; wing at 10-degree initial effective angle.

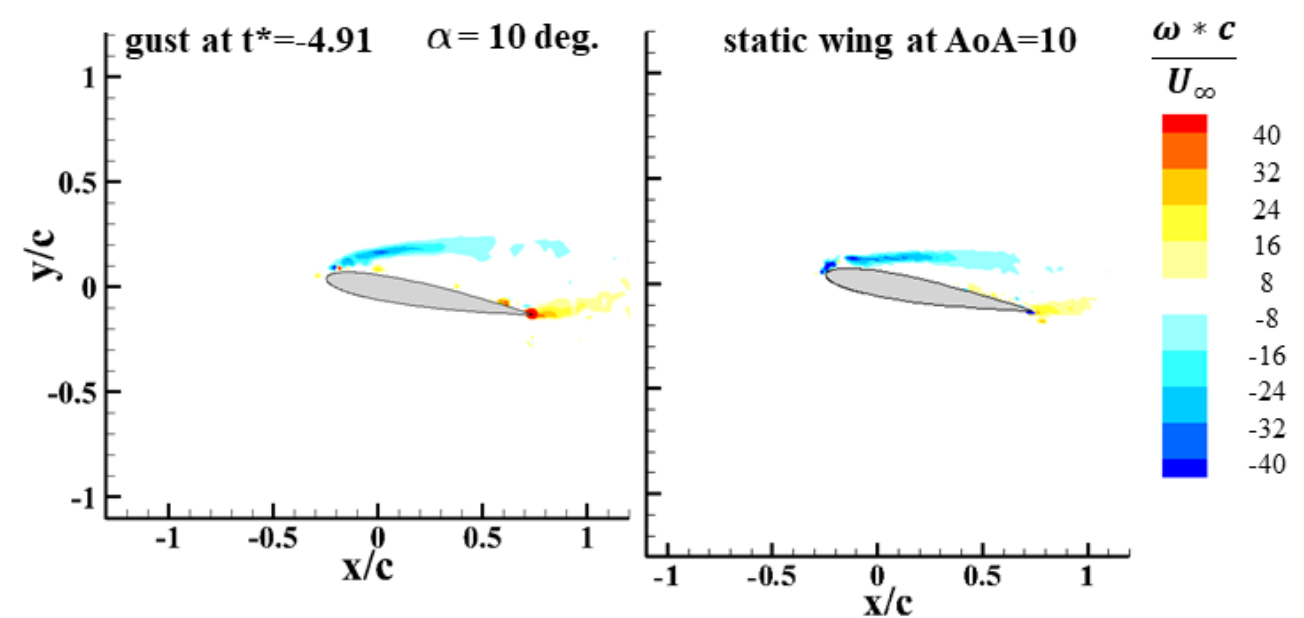

Figure 91: Early mid-power-gust/static wing comparison; 10-degree initial effective angle. 


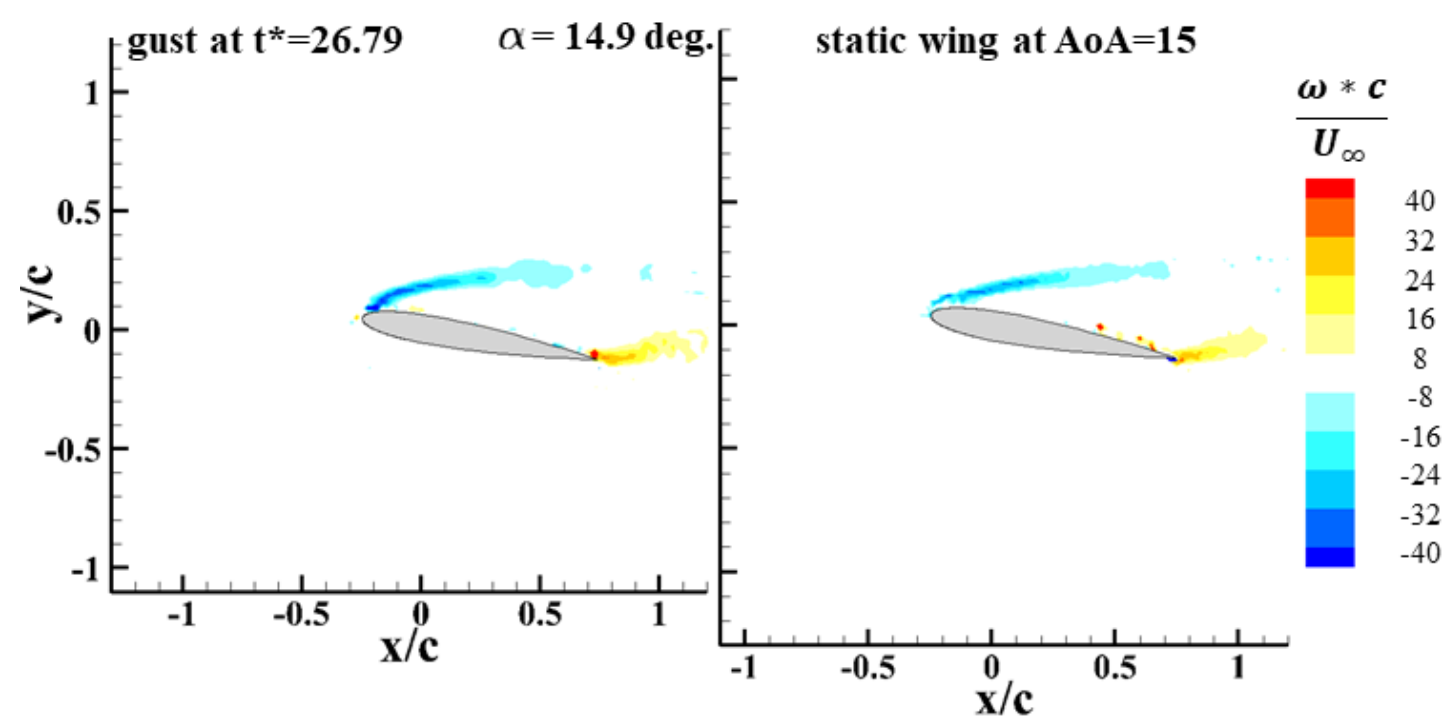

Figure 92: Late full-power-gust/static wing comparison; 10-degree initial effective angle.

As was expected, these results show little growth in the vorticity field throughout the gust actuation process. Since the gust's effects are so low, these are some of the closest resembling comparisons done so far. The wing with an initial 0-degree effective angle at a convective time that is nearing the gust's full-strength condition is comparable to a 5-degree no-gust wing. This continues the trend found in the mid power gust comparisons. Clearly as the gust strength decreases, the wing matches more closely to a simple static angle change for the wing. However, even at this low gust strength, the curvature of the vorticity from the leading edge of the wing is still visible. Therefore, it is shown that even in very low gust ratios this curvature would still need to be accounted for in modeling or control work.

\subsection{Pitching a Wing to Simulate Gust}

\subsubsection{0-degree Initial Angle Pitch Maneuver}

The results of the gust-wing interaction suggested that few similarities exist between static results and the gust interaction, especially at high gust ratios. The next 
step beyond a static wing comparison is the comparison with an actively pitching wing. It was thought that the dynamic nature of the pitch may show a higher correlation to the dynamic gust. To simulate the full-power gust case, the wing was pitched from 0 degrees to 13 degrees in 0.856 seconds, or 10.7 convective times. This was done to match both the flow angle achieved and the time it took for the gust to fully develop in an empty tunnel. This resulted in a dimensionless pitch rate of $\Omega^{*}=\dot{\alpha} \mathrm{c} / 2 \mathrm{U}_{\infty}=0.01$. For reference, dynamic stall studies typically have dimensionless pitch rates of $\Omega^{*}>0.05$. The linear pitch profile for this study can be seen in Figure 93.

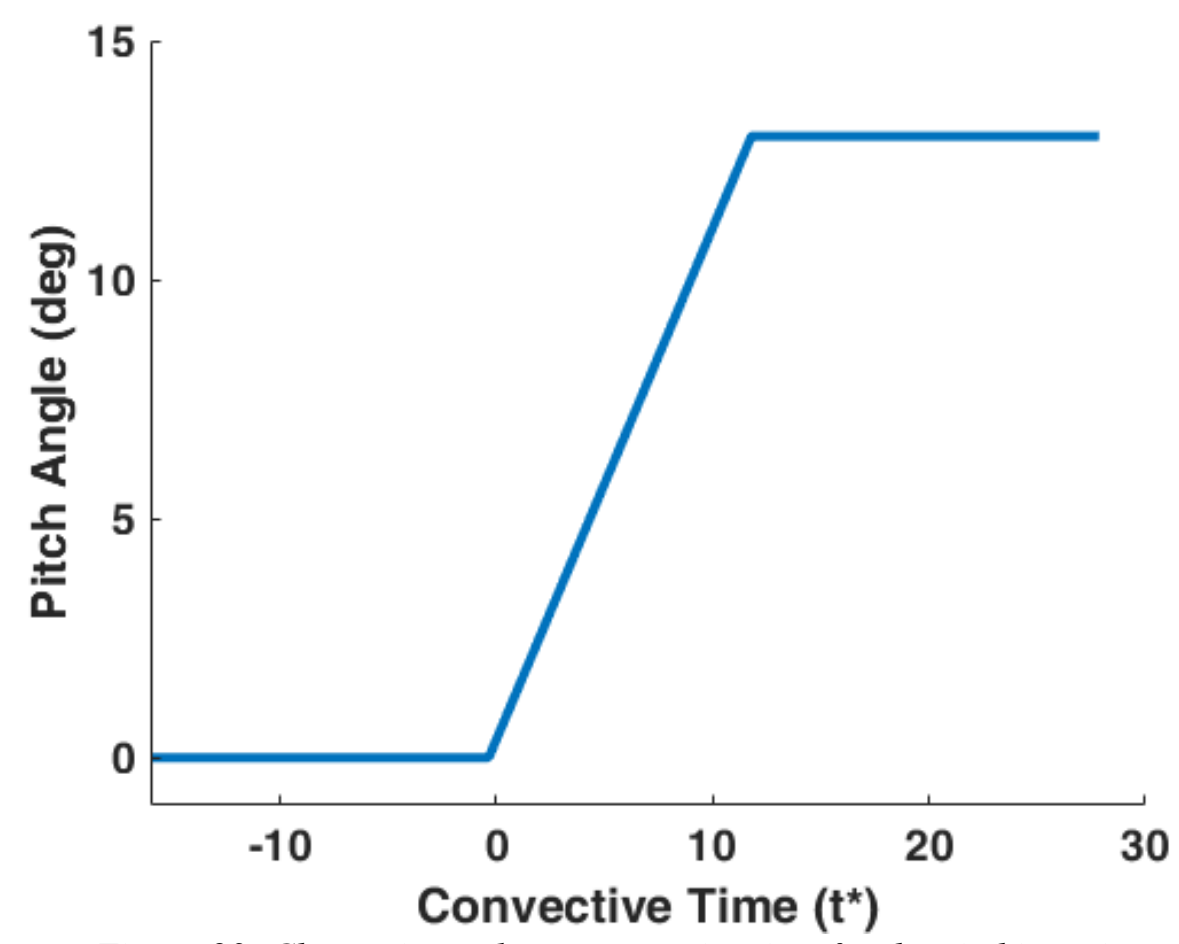

Figure 93: Change in angle vs. convective time for the pitching wing.

Selected time steps extracted from this pitch motion case can be seen in Figure 94. Note that the data presented in Figure 94 is in the wing frame of reference for ease of comparison with the gust results. 


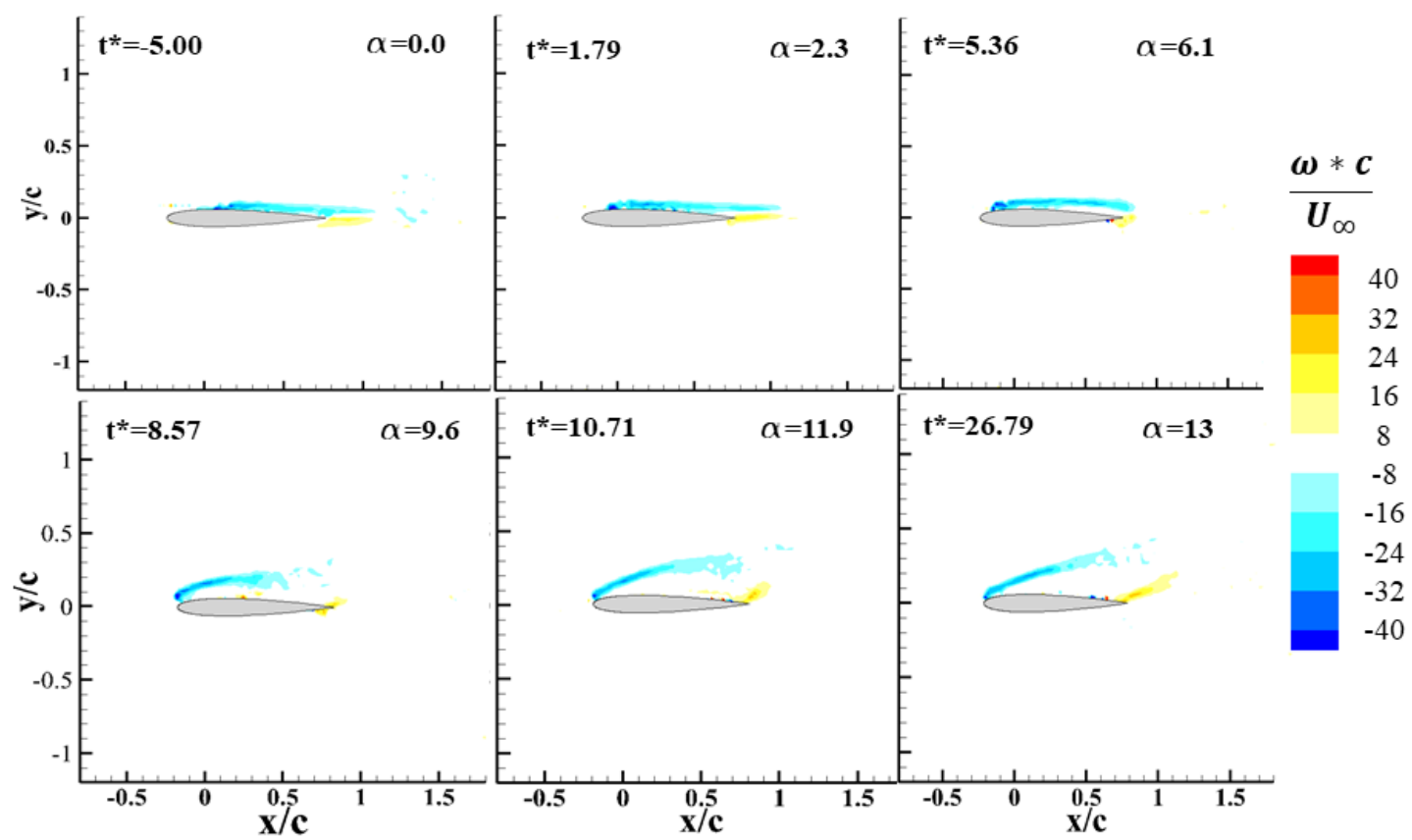

Figure 94: Wing pitched from 0 to 14 degrees to simulate full power gust.

The first few time-steps shown in Figure 94 display the vorticity growing as expected, with the flow remaining attached to the wing through $t^{*}=5$. The flow fully separated at roughly $t^{*}=6.5$, which was earlier than the separation time found for the gust interaction, where separation occurred around $t^{*}=11$. This indicates that the pitch motion does not incur the delay in vorticity growth shown in the gust-wing interaction cases. The separation in the pitching case then increased until the motion stopped, and a steady state was reached at $t^{*}=12.2$. It was shown that the wing stalled somewhere between 5 and 10 degrees, so the final state of this pitched wing is well into the stalled region. However, while the wing ended in a deep stall state, this type of deep stall was not seen in the gust actuation results.

Figure 95 shows a comparison between the last time of the gust actuation scenario and two selected times from the pitching wing scenario. One of these matches the same convective time and the other is an arbitrary time that appears to contain similar 
characteristics between the gust case and pitching wing case. When compared at the same angle and convective time $\left(t^{*}=26.25, \alpha=13\right)$, the data sets looked significantly different. However, this late time in the gust actuation compared particularly well with a lower time and angle ( $\mathrm{t}^{*}=8.57, \alpha=9.87$ degrees $)$ for the pitching wing.

As was previously seen, there was a delay between the opening of the slats and the time it takes for the gust to reach the wing's position. This delay was observed to be 1 convective time from Figure 56. For clarity, the wing gust case is being shown in term of $\tau$ which is $t^{*}+1$.

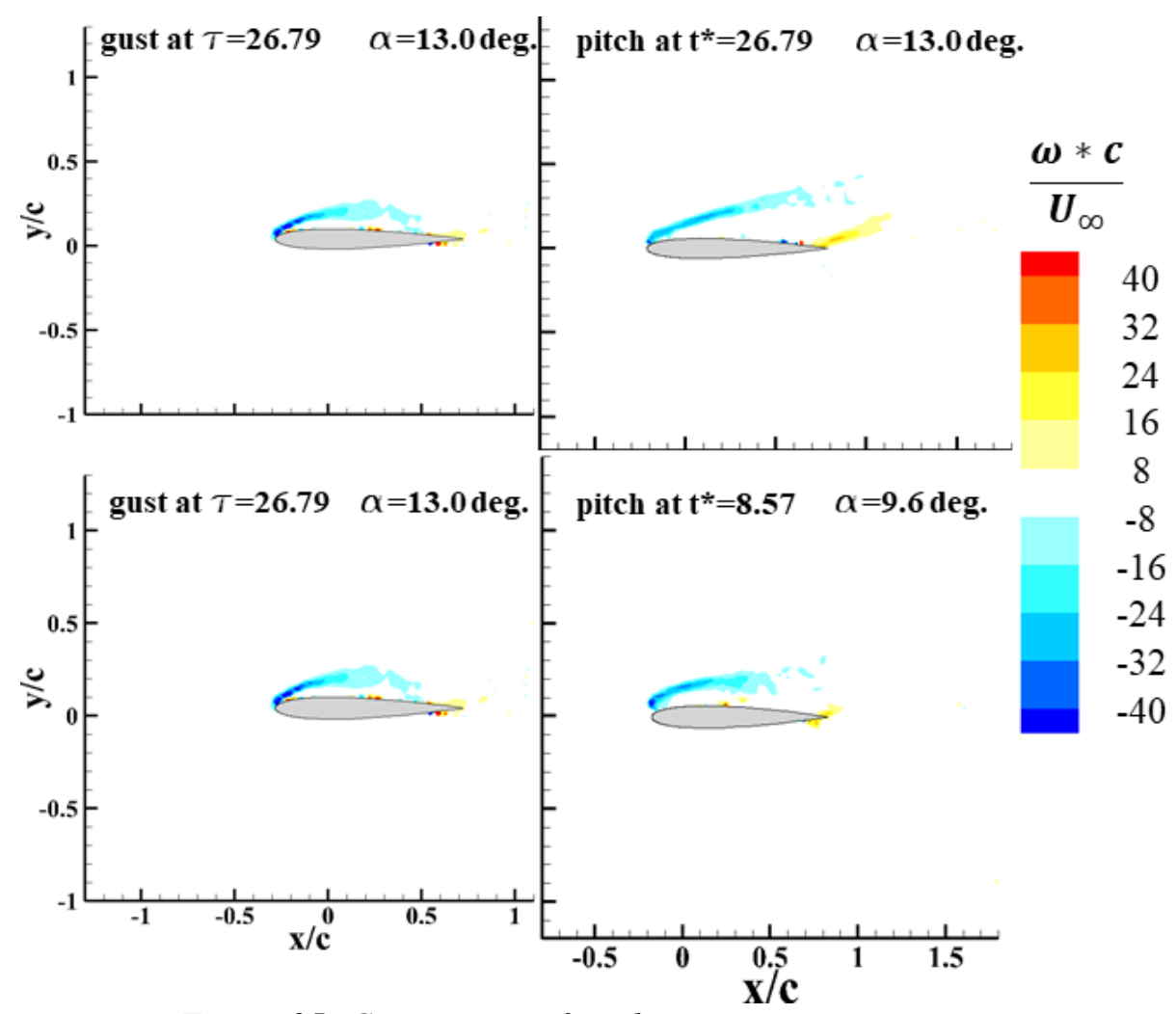

Figure 95: Comparison of pitching wing to wing in gust.

When both results are analyzed at $t^{*}=26.79$, there was practically no similarity between the gust actuation and the pitching wing. However, the results from the gust actuation case at $t^{*}=26.79$ matched closely with those of the pitching wing at $t^{*}=8.57$; 
more so than for any of the steady state comparisons. For the pitching wing at $t^{*}=8.57$, the vorticity near the pitching wing followed the curved path that was seen in the gust wing at $t^{*}=26.79$. However, the angle of the wing was lower than the estimated flow angle. Additionally, these results suggest that the pitching wing case can evaluate the transient gust behavior but not match the fully developed gust. This effect could be caused in multiple different ways. The wing may affect the intensity of the gust, the method for estimating the effective angle may be ineffective without mapping the entire flow field, or the pitch rate estimated may be incorrect to make an ideal comparison. With these ideas, some hope is had that the gust-wing interaction can be compared to that of a pitching wing up until both reach a steady state by adjusting the final pitch angle but estimating that angle remains a challenge.

In an attempt to better determine the exact flow angle around the wing at the end of the gust interaction, the flow angle and gust ratio for both the initial state and the final state with respect to the wing are shown in Figure 96 and Figure 97, respectively, for the gust-wing interactions at the marked convective times.

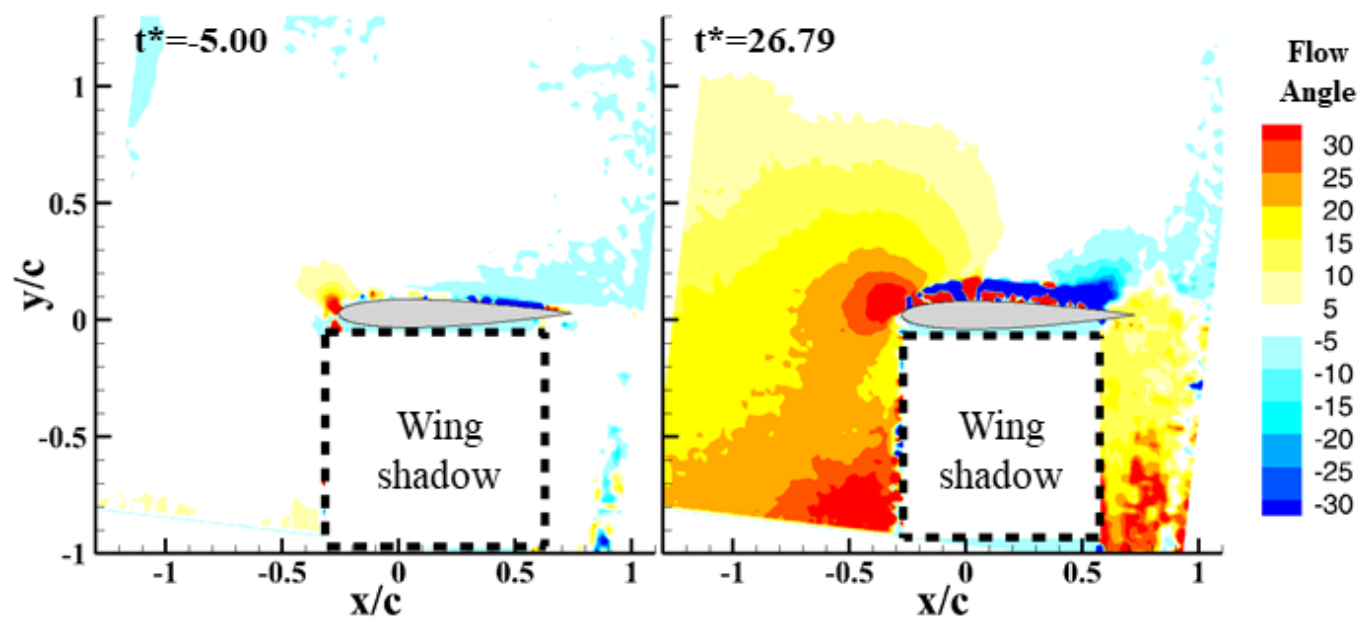

Figure 96: Flow angle comparison for a wing pre-gust and in a steady gust. 


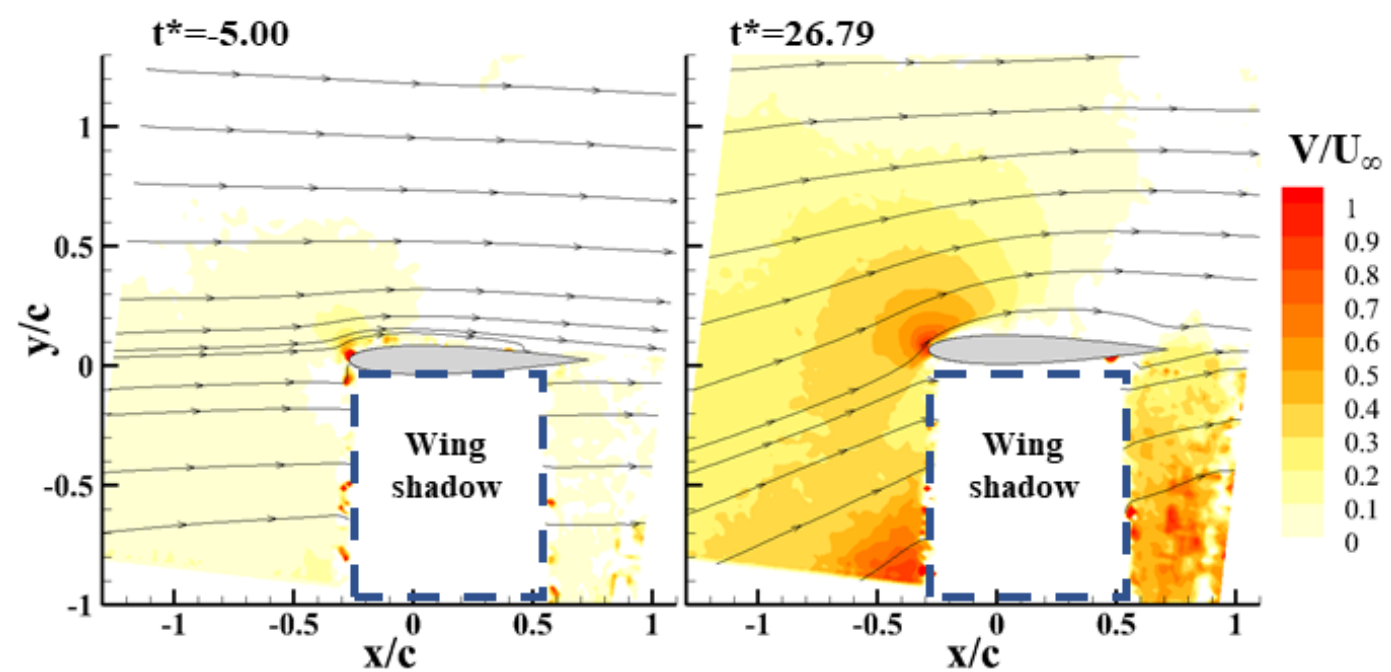

Figure 97: Gust ratio comparison for a wing pre-gust and in a steady gust.

At $t^{*}=-5.00$, the majority of the incoming flow with respect to the wing was at approximately 0 degrees and has a 0 -gust ratio; reconfirming that the effective angle was calculated correctly for the initial pre-gust condition. The images at $t^{*}=26.79$ show that the flow with the wing in it was not as simple to interpret as an empty tunnel. This is obvious when one stops to consider the effect the wing has on both the vertical flow, by acting as a blockage, and the horizontal flow, by speeding it up over the top half of the wing; plus, the effects stall has on these properties by the wing surface. These effects make it difficult to accurately estimate the incoming flow angle once the wing is in the tunnel, but this effect is likely similar to that of a real-world wing interacting with a gust. A similar deflection of the real-world gust would be expected, and these results suggest that further understanding of the interaction is still needed.

Until now, the flow angle was being determined based on the $\arctan \left(\frac{V_{\text {gust }}}{U_{\text {gust }}+U_{\infty}}\right)$. However, $\mathrm{U}_{\text {gust, } \max }$ is known to be only about $8 \%$ of $\mathrm{U}_{\infty}$. This study is mainly focused on the vertical gust effects and not so much the horizontal gust effects. When recording static and pitching wing data for comparison with the gust, the freestream flow was not 
increased to match $\mathrm{U}_{\text {gust }}+\mathrm{U}_{\infty}$ and was simply left as $\mathrm{U}_{\infty}$. With this in mind, a new fullpower flow angle plot was created that evaluated the flow angle as $\arctan \left(\frac{V_{\text {gust }}}{U_{\infty}}\right)$. The result of this, seen in Figure 98, is a flow angle change of closer to 10 degrees, from 5.4 degrees prior to the gust actuation to 15.4 at the full-strength case. This change occurred over approximately 8.5 convective times. Using this estimation for the effective flow angle better represented the results of Figure 95, where the estimated flow angle change was previously 13 degrees. If the flow angle was estimated using the results of Figure 98 instead, this comparison would have been $a=10$ degrees compared to $a=9.87$ for the pitching case. Clearly the estimation of the flow angle that the wing experienced is key to making accurate comparisons despite the challenges associated with that estimation.

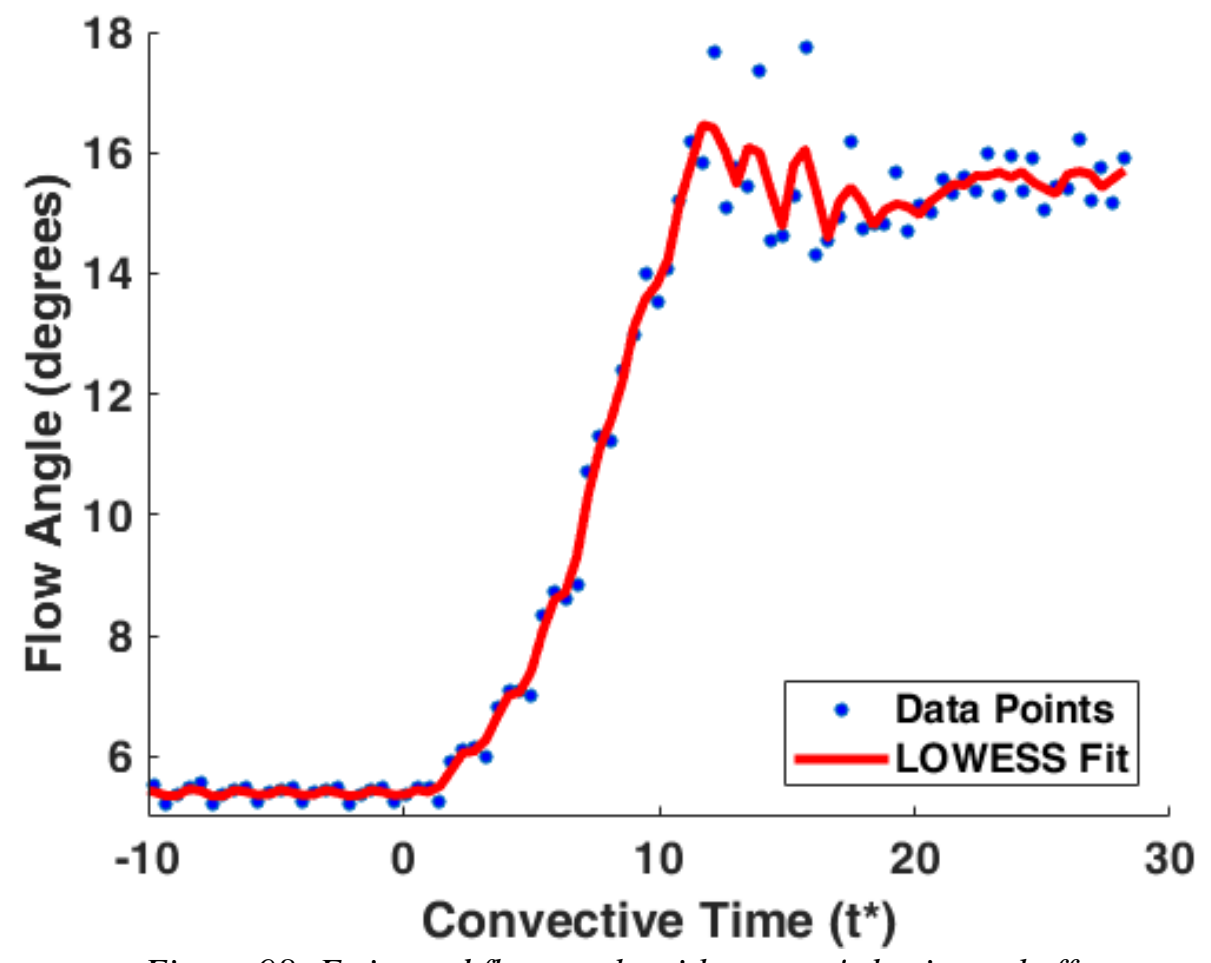

Figure 98: Estimated flow angle without gust's horizontal effect. 
To see if this new estimation was more effective as a pitching profile, a test case was run pitching the wing from 0 to 10 degrees in 0.656 seconds, or 8.5 convective times. This is practically the same pitch rate as before $\left(\Omega^{*}=0.01\right)$, just ending the motion 3 degrees earlier. The profile for this pitching motion can be seen in Figure 99; with the collected images from this new pitch case and the associated comparisons to the gust shown in Figure 100 through Figure 103.

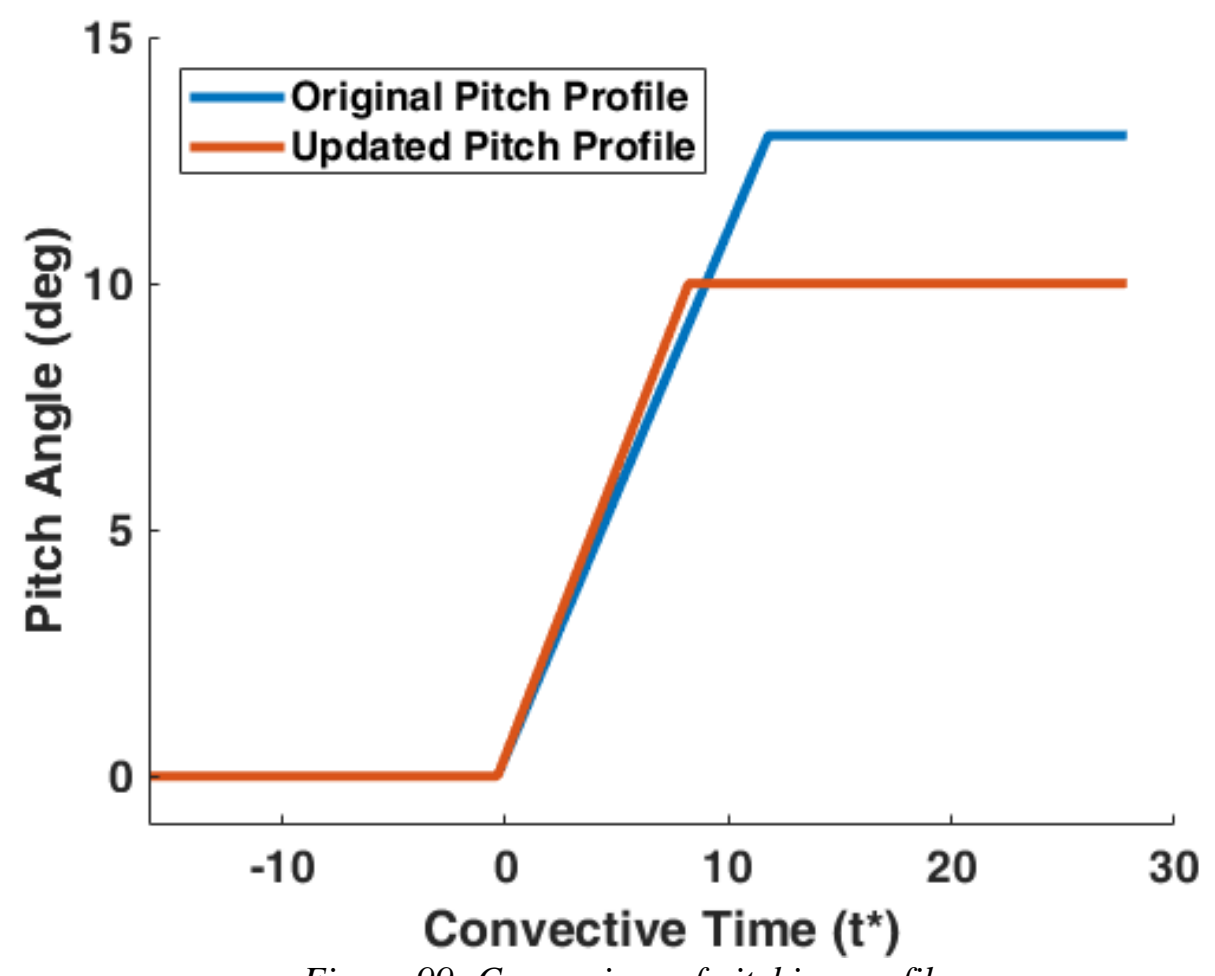

Figure 99: Comparison of pitching profiles. 


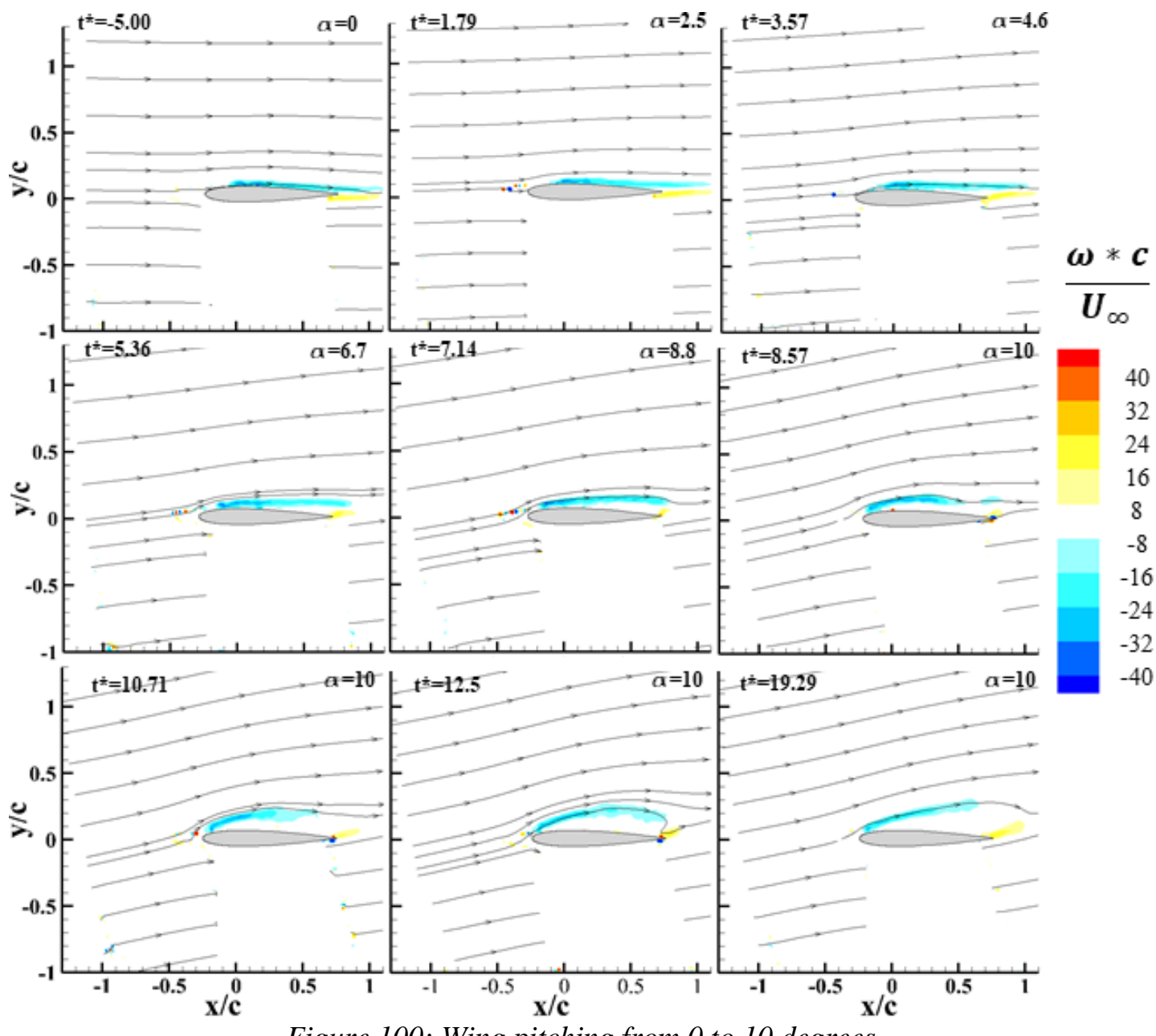

Figure 100: Wing pitching from 0 to 10 degrees.

Examining the early times of the new pitch maneuver showed that the wing behaved as expected with equal trailing sheets of vorticity being shed from the top and bottom surfaces. At $t^{*}=3.57$ the suction side vorticity began to lift off the wing beginning near the trailing edge. This process continued until roughly $t^{*}=8$, after which the vorticity began to curve toward the trailing edge similar to the wing-gust interaction. While the pitching motion stopped just before $t^{*}=8.57$, the flow continued to bend toward the trailing edge and did not enter a steady-state stall condition until nearly 2 convective times later $\left(t^{*}=10.71\right)$. This indicates that the flow structures 
created by the pitching motion stay present around the wing after the pitching motion completed, but a fully-stalled condition would eventually occur unlike in the gust case.

A comparison of this pitching motion and the gust results is shown in Figure 101, Figure 102, and Figure 103. A set of times from the beginning $\left(\mathrm{t}^{*}=1.79\right)$, middle $\left(\mathrm{t}^{*}=7.14\right)$, and end $\left(\mathrm{t}^{*}=10.71\right)$ of actuation process were used for comparisons. The wing angle is only shown on the pitching wing case as to not create confusion between the previous estimated effective angles for the wing-in-gust case.

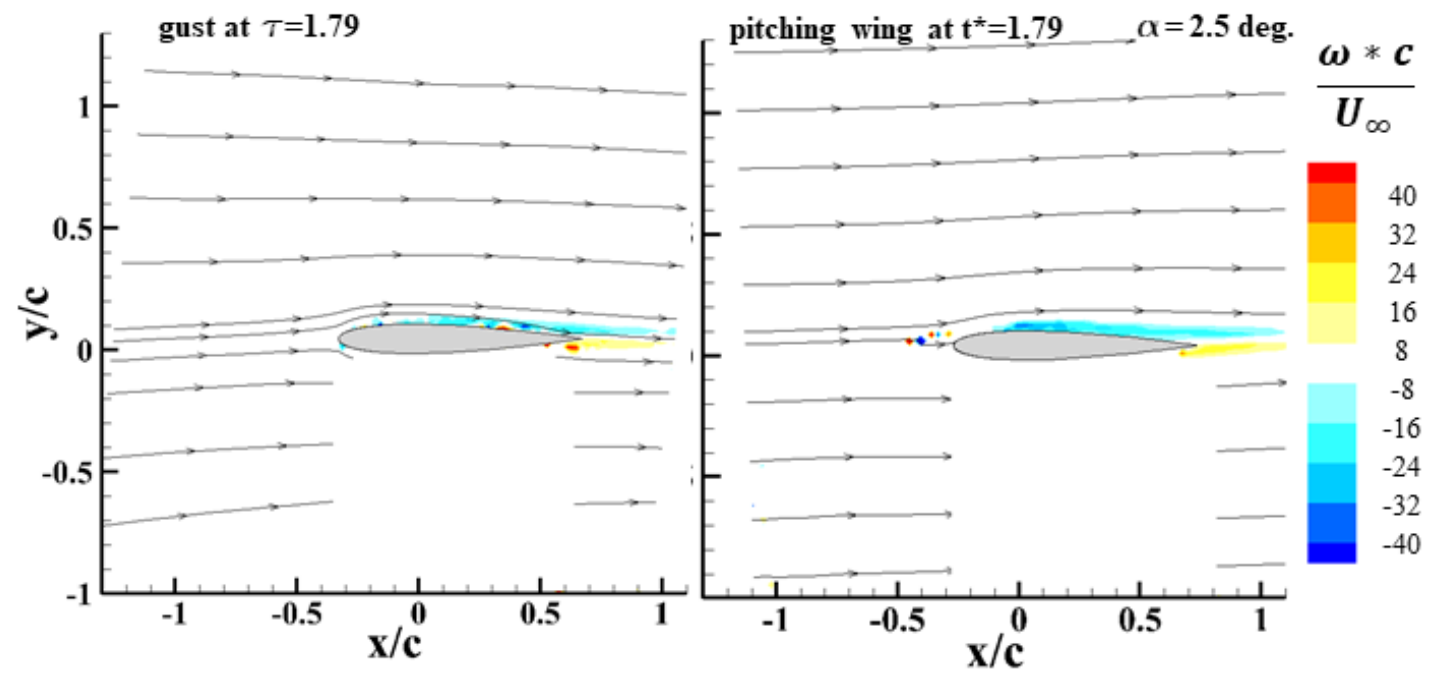

Figure 101: Early gust-pitch comparison at 0-degree initial effective angle.

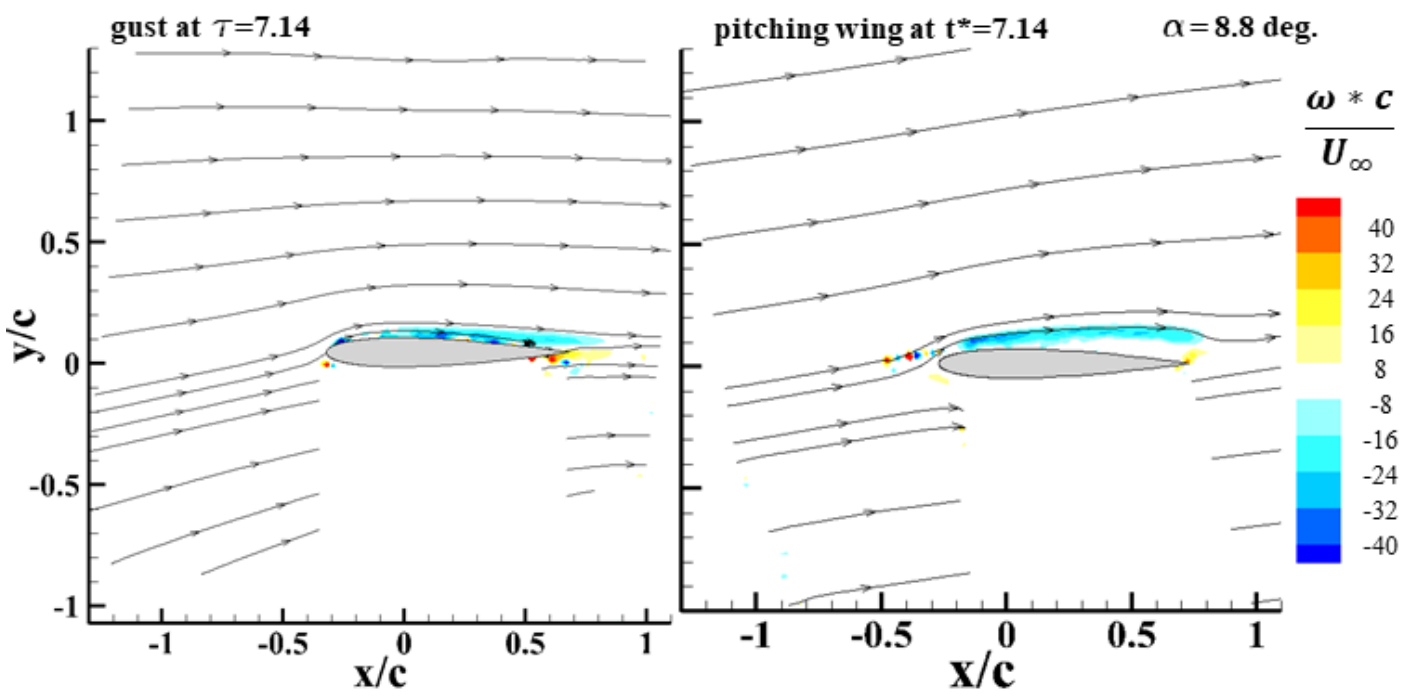

Figure 102: Mid gust-pitch comparison at 0-degree initial effective angle. 


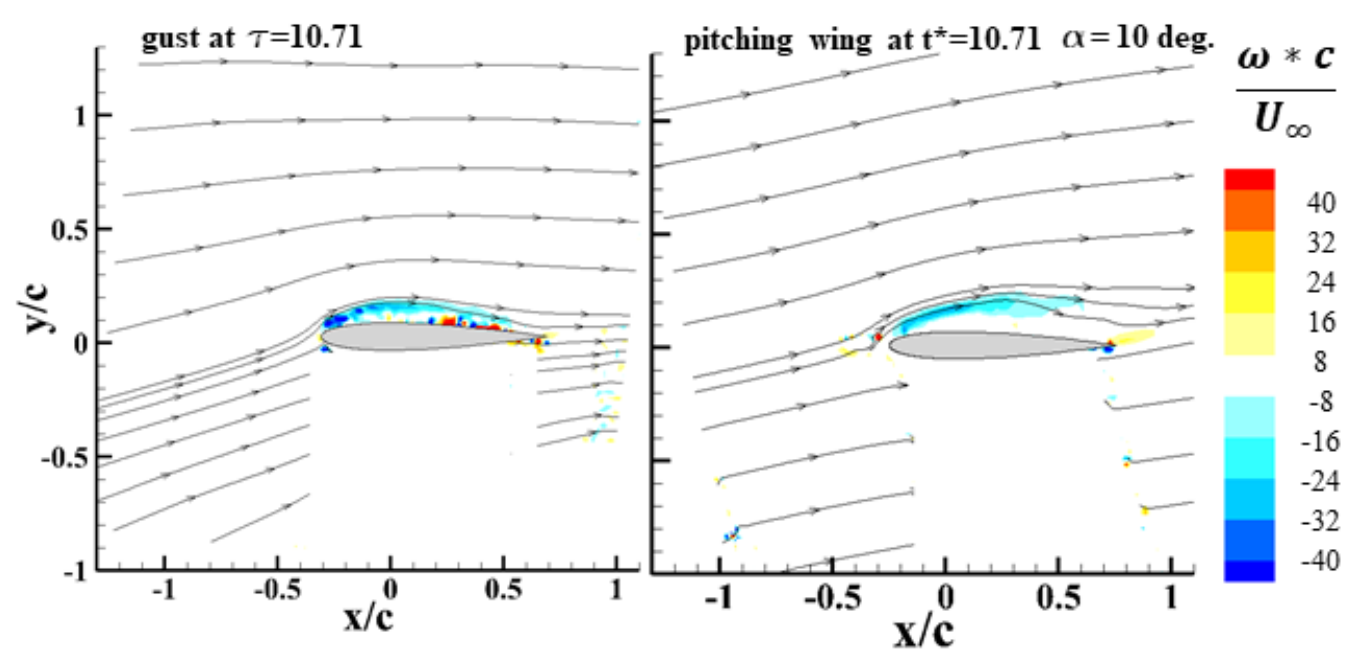

Figure 103: Late gust-pitch comparison at 0-degree initial effective angle.

The early time in the pitching motion lined up well when compared to the gust. About 5 convective times later, Figure 102, the vorticity strength across the wing in both cases was quite similar, but the path the vorticity follows is different between the gust and pitching wing cases. In this case, the flow had separated from the trailing edge on the pitching wing but remained attached during the gust interaction. However, 3.5 convective times later, for the final comparison in Figure 103, both cases had a similar flow curvature with the flow nearly reattaching at the trailing edge. The vorticity was more separated for the pitched wing when compared to the wing in the gust, but the behavior of the two was now similar at the same convective time.

Overall, these results showed that a pitch-and-hold motion profile can mimic the unique reattachment characteristics of the gust. This strongly suggests that the gust can be recreated with a pitching motion that accurately reflects the effective angle seen by the wing in this type of gust. While there is still some difference observed between the gust and pitching case, alignment of similar flow fields at similar convective times indicates that further refinement of pitch rate and final pitch angle could accurately 
recreate the flow field in a transient way. However, the first few convective times would likely not align perfectly since it was shown here that the arced vorticity path does not occur in the pitching wing case during the first few convective times of motion for this particular pitching profile. Also, after the pitching motion stops the pitching wing would enter a traditional stall state which would not recreate the steady gust. Further study should be done to refine these comparisons and identify the pitching profile that most accurately reflects the gust, although that profile may not be linear as was assumed here.

\subsubsection{5 and 10-degree Initial Angle Pitch Maneuvers}

Two other wing pitching cases are presented here, although both were operated using the 13-degree pitching motion as opposed to the 10-degree motion. Note that the correction from the 13-degree pitching case was only done on the 0-degree AoA wing as that analysis was conducted after recording the pitching motion for 0,5 , and 10 initial angles. The data presented in this section is for a wing starting at 5 degrees and pitching to 18 degrees, and starting from 10 and pitching to 23 degrees, both over 10.7 convective times. The first of these cases, the one starting at 5 degrees, can be seen in Figure 104. 


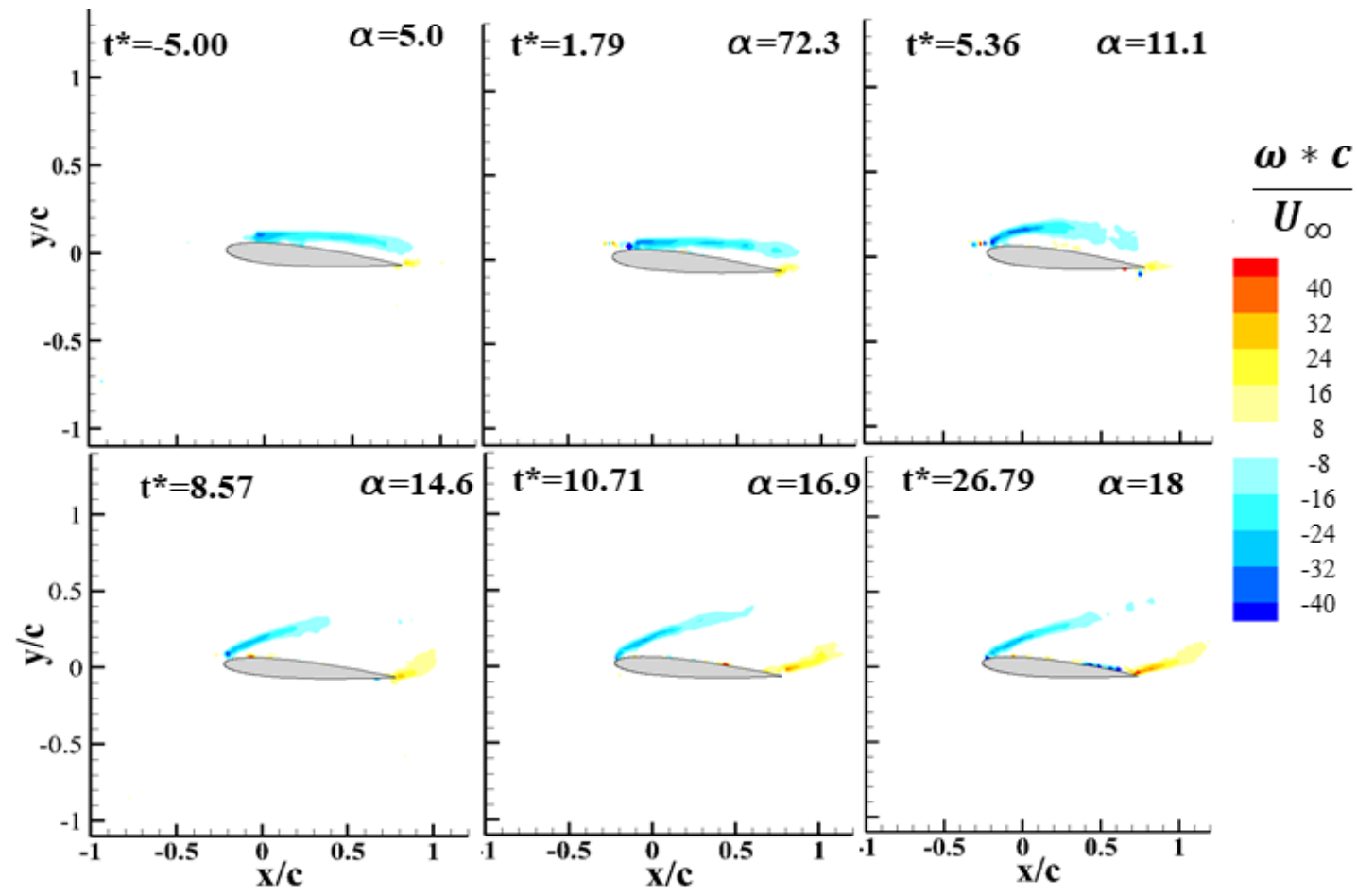

Figure 104: Wing pitching from 5 to 19 degrees to simulate full power gust.

The results shown in Figure 104 were comparable to the previous 13-degree pitch motion case. The flow separated earlier in the pitch maneuver because the wing started from a higher angle of attack initially. The same result from the previous pitch case that involved a 13-degree angle change was expected: the wing's final pitch state results in vorticity that is far more separated than the gust case. This became obvious in the side by side comparison shown in Figure 106 and Figure 105. 


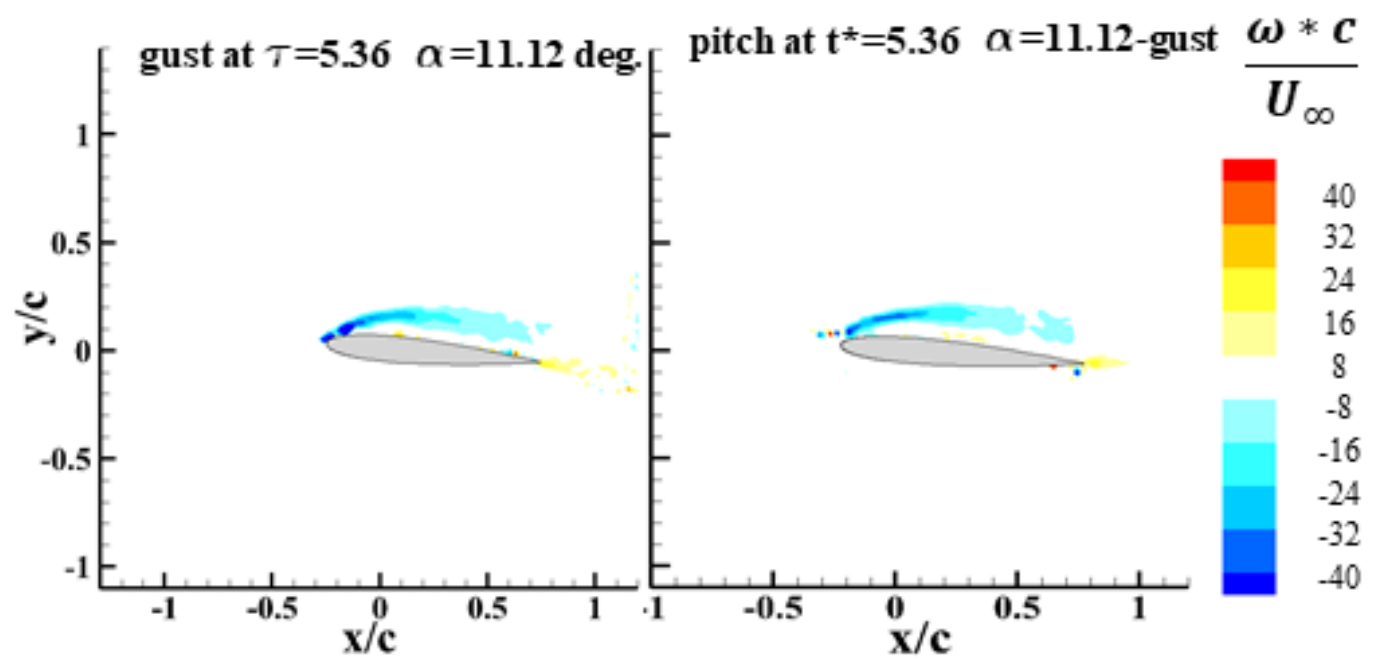

Figure 105: Mid gust-pitch comparison at 5-degree initial effective angle.

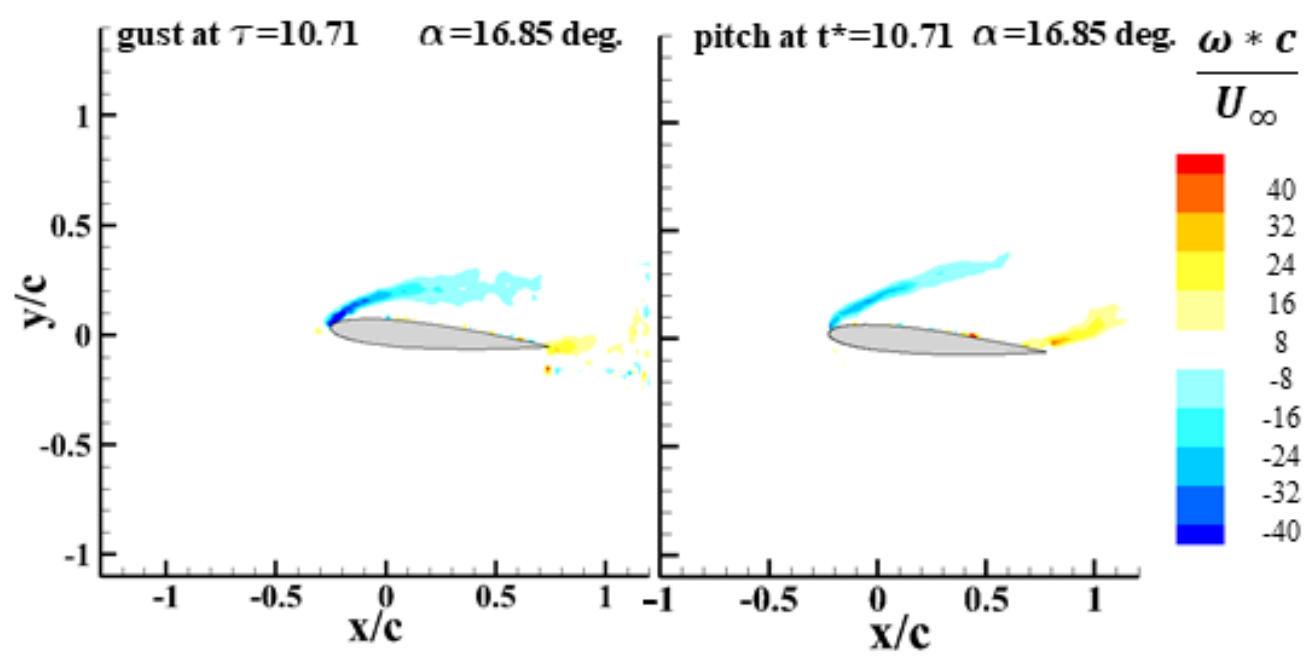

Figure 106: Late gust-pitch comparison at 5-degree initial effective angle.

Figure 105 shows that towards the beginning of the motion, the vorticity lines up nearly exactly at matching convective times. This is a striking resemblance and somewhat unexpected as it was assumed based on the results from the 0 to 13-degree gust-pitch comparison shown in Figure 95, that the wing would appear to be well over pitched for this case. This result stands in high contrast to the results found in Figure 102, which was for the reduced peak pitching motion and showed poor correlation at intermediate time. It is believed that either the difference in the pitching profile between 
the two cases or the difference in starting angle of attack is what is cause this vorticity curvature to form in one case but not the other so early in the motion. Figure 106 shows that the difference observed near the end of the pitching motion increased. This seemed to be a result of the wing reaching a deep post-stall condition much sooner, and thus transitioning toward the static stall case faster. This result is further evidence that the transient nature of this comparison must be better understood when attempting to compare gusts to pitching motion.

The final pitching case, pitching from 10 to 24 degrees, can be seen in Figure 107. The comparisons between this pitch case and the gust case can be seen in Figure 109 and Figure 108.

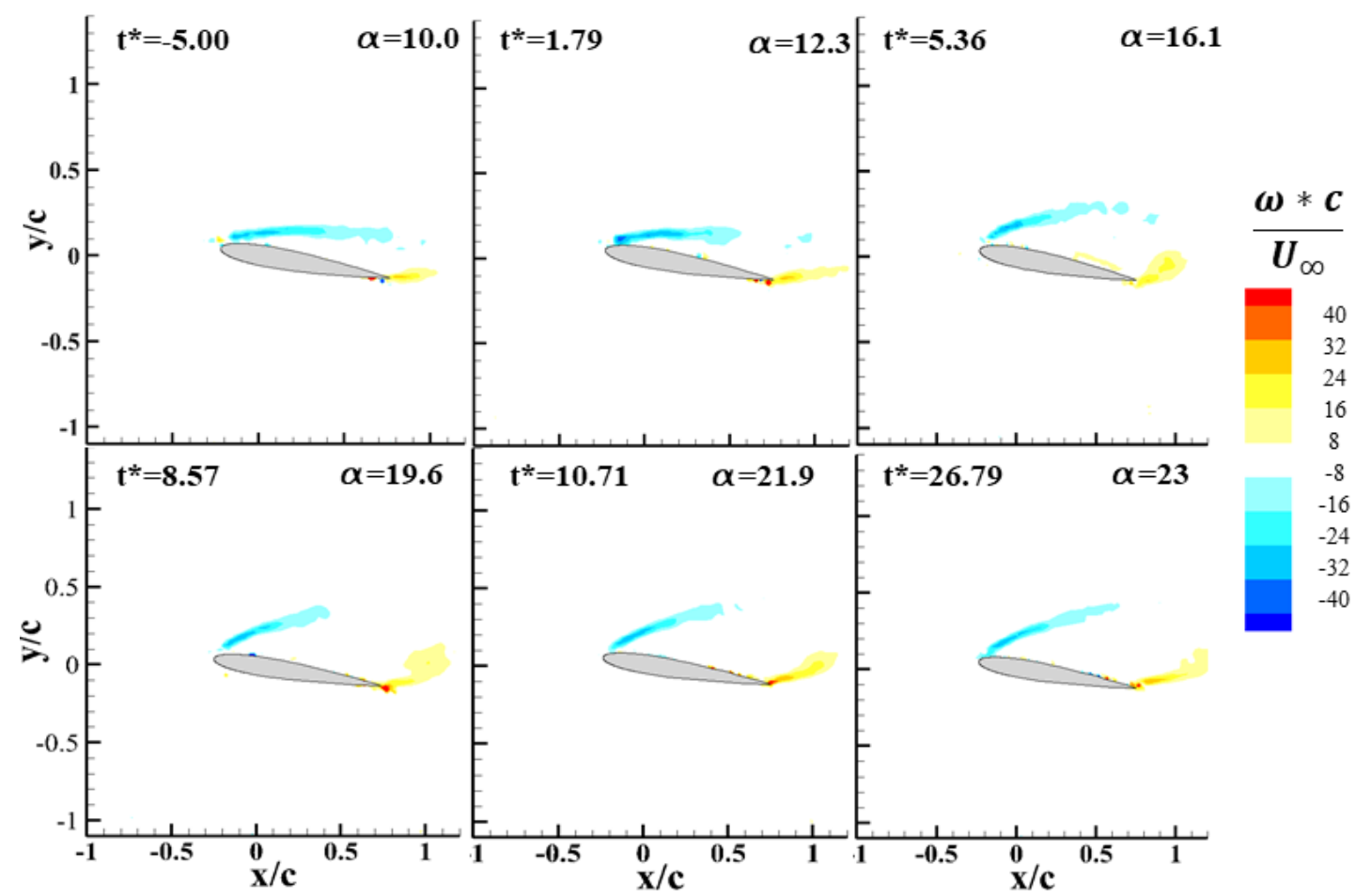

Figure 107: Wing pitching from 10 to 24 degrees to simulate full powered gust. 


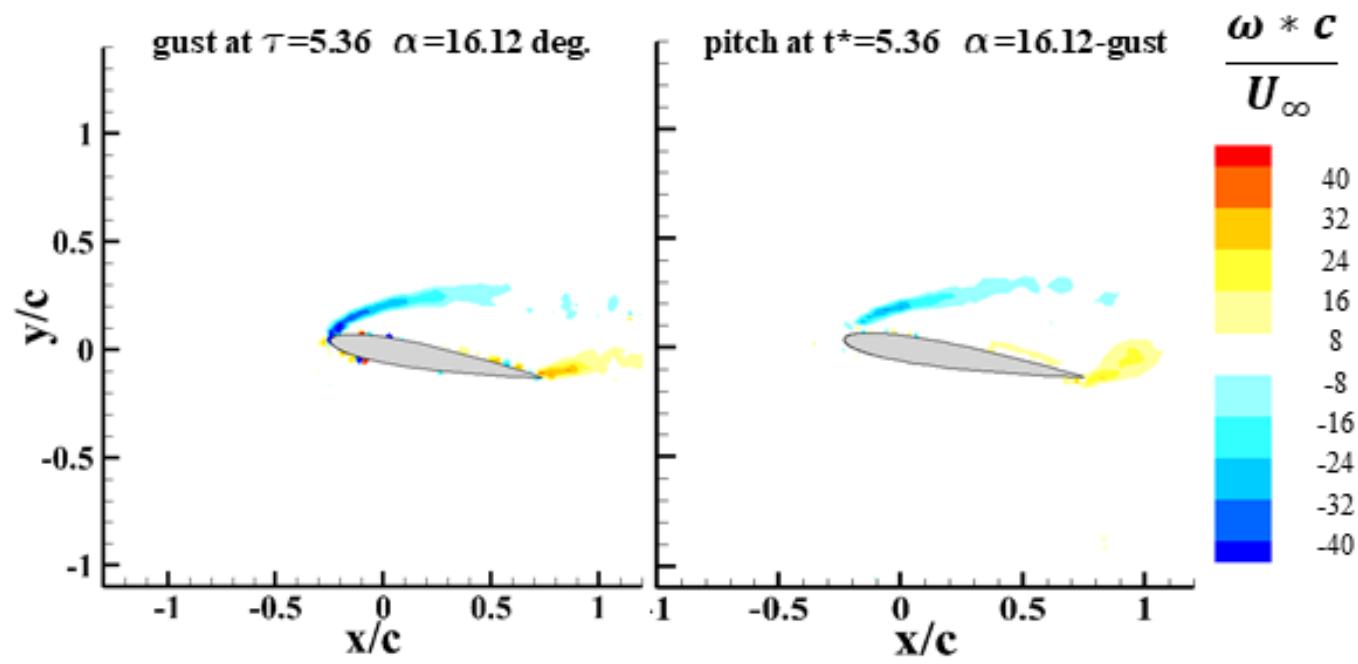

Figure 108: Mid gust-pitch comparison at 10-degree initial effective angle.

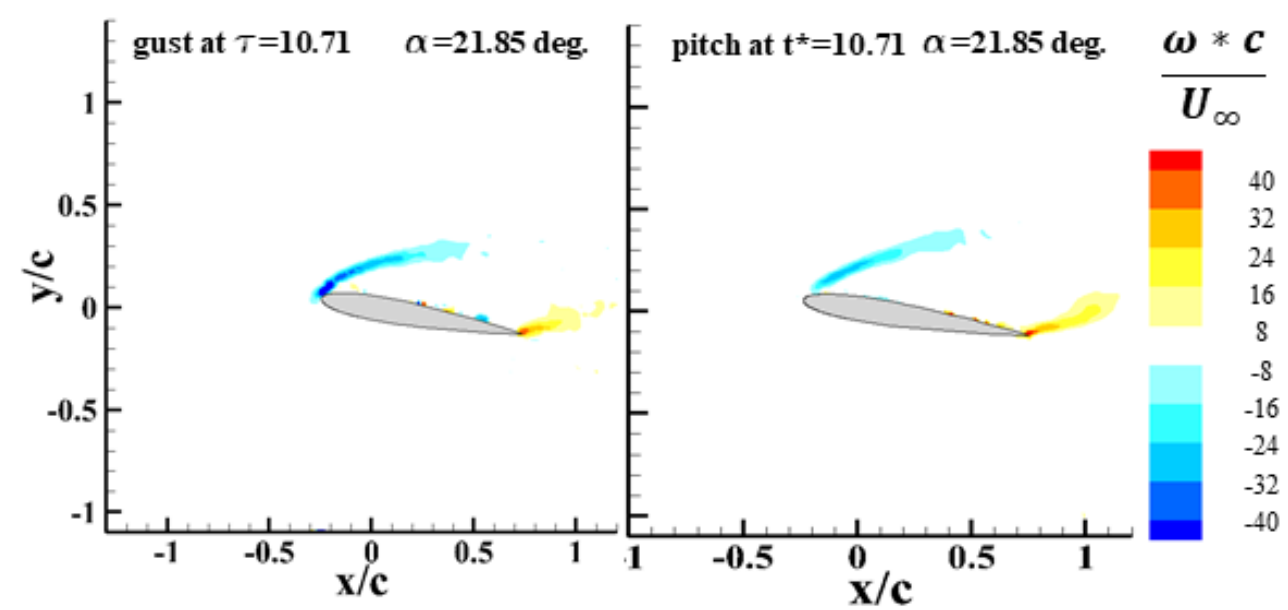

Figure 109: Late gust-pitch comparison at 10-degree initial effective angle.

The data compared here was very similar, but not unsurprisingly so. While the data did not perfectly align, the strength of the vorticity and the path it followed matches well between the pitching motion and the gust-actuated case. It was previously shown in the static wing comparisons that at initially high angles of attack, the gust-wing interaction was dominated by the stall condition and not the gust interaction. Therefore, by adding the dynamic nature of the pitch and knowing how the pitch maneuver previously curved the flow toward the trailing edge, it could have been predicted that these profiles would look very similar to one another. This further suggests that if one 
has the ability to determine the proper pitch rate and maximum pitch angle achieved, a gust interaction can by simulated with a pitch to some high level of accuracy past a certain convective time and prior to the steady state solution.

No comparison between the low power and mid power gust cases were made for the pitching wing. Different pitch rates would have been used to better match the flow angle changes seen in both of these cases. But it was again thought that similar results could be drawn from these comparisons as before and these tests would not further the information present in this study.

\subsection{Chapter Summary}

This chapter stepped through the evolution of the gust as it entered the wind tunnel and affected the flow field. An empty wind tunnel was first analyzed at all three gust fan powers. The growth of freestream $\left(\mathrm{U} / \mathrm{U}_{\infty}\right)$, gust ratio $\left(\mathrm{V} / \mathrm{U}_{\infty}\right)$, and flow angle were evaluated and tracked as a function of convective time. For the full power case where the peak gust magnitude was over $30 \%$ of freestream, the horizontal flow speed, gust ratio, and flow angle grew rapidly before oscillating around a steady state value. These values were slightly above the fully developed flow values indicating there was some settling after the ensemble averages were recorded. There was some interesting behavior observed later in the actuation process for the mid and low power gust cases. It was shown that two separate growth regions existed for freestream, gust ratio, and flow angle with an early acceleration followed by a slower growth up to steady state, which appeared to occur after the ensemble average peak recorded time.

A wing was placed in the tunnel and the effect the gust had on a wing at various effective angles of attack and various gust powers were analyzed. During the 
interaction of the gust with the wing, it took an extra 5 convective times for the wing to reach the fully developed state observed in the characterization, although this was somewhat expected and considered reflective of a wing entering a real-world updraft. The data from this interaction were also compared to that of a steady wing at various angles of attack and with no gust present. Similarity between the vorticity strength for both cases was seen, but the separated vorticity path varied between the gust and static cases at pre-stall static angles. The similarity between the vorticity for both cases increased as the initial angle of attack was increased. This suggests that when a wing enters a gust already stalled, the change in the flow field is not as large; and therefore, it more closely resembles that of a wing at fixed angles.

The gust was further compared to a wing pitched with a motion profile similar to the characterized gust. This was done at a constant pitch rate based on the evaluation of the flow angle characterization in the near wing region, but with two different peak angles. One peak pitching angle (13 degrees) was calculated accounting for the change in freestream velocity and vertical velocity induced by the gust, while the other (10 degrees) neglected the freestream changes. During the gust encounter, the flow reattached near the trailing edge of the wing, but that was not always the case in the pitching motion data. The gust-wing interaction better resembled the smaller peak pitching angle motion profile as it.

Near the beginning of the pitching motion, the vorticity separated from the trailing edge forming a vorticity pattern similar to that of a static wing at low angle of attack. However, about halfway through the pitch motion the vorticity began to curve and closely resemble the wing-in-gust case. These results strongly suggest that some 
pitching motion profile can be found that will mimic the flow field that occurs when a static wing encounters a vertical gust. While the results of the pitching motion showed close correlation, the transient gust-like behavior evolved to the fully stalled state after a short time. Knowing this, for a step-function style vertical gust a pitch maneuver is unlikely to represent the gust at large times. Gust studies should generally be performed with a gust simulating device (i.e. moving fluid) and not a moving model simulation. 


\section{Chapter 6: Conclusion}

\subsection{Summary}

Due to their size and flight speeds, MAVs and small drones are extremely susceptible to gust encounters. Therefore, it is extremely important for researchers to study the aerodynamics governing gust-wing interactions at the most fundamental level. There have been many gust testing apparatuses over the previous century, but very few fundamentally new designs have been implemented over the last 20 years. The current study began by determining what a new gust apparatus might include to improve upon past concepts and how it would be designed. Once some design criteria were established, a novel gust generator was created and installed in a low speed, low turbulence wind tunnel.

This novel gust design involved the use of an off-the-shelf exhaust fan ducted through the floor of a wind tunnel to create and study a transverse gust interaction. A 3D printed expansion nozzle was installed at the interface between the gust system and the wind tunnel in an attempt to spread the flow evenly across the bottom of the tunnel. An exhaust valve was installed on the top of the wind tunnel and connected back to the fan via flexible ducting. PIV was used to study the gust freestream interaction and this closed loop gust generator ensured that PIV seeding particles would be present in the gust itself.

After the gust-generating system was installed, the full operational space for the gust was characterized. This included putting the gust in 4 positions in the streamwise direction of the tunnel and testing 3 different gust powers at each location. Since the wing-mounting system could not be moved in the tunnel, the optimal location for the 
gust to achieve the best interaction at the fixed wing location was determined. The full power case at the furthest upstream position, Position 1, was shown to create the best results for future tests.

Once this location was chosen, the effect of gust actuation (i.e. startup) was studied. Ensemble averaged PIV was used to study the flow field during this actuation. The changes in freestream $\left(\mathrm{U} / \mathrm{U}_{\infty}\right)$, gust ratio $\left(\mathrm{V} / \mathrm{U}_{\infty}\right)$, and flow angle were analyzed versus nondimensional time for each of the three gust powers at Position 1. All three cases showed a quick rise in the measured characteristics to a settling point. At this settling point, the full power cases oscillated, but the mean value was generally consistent with data from the steady-state characterization. For the low and mid power cases, the gust properties continued to rise indicating these powers were not as well suited for the current study.

Once the actuation process was studied, a wing was placed in the tunnel and the gust-wing interaction was observed during and after the gust startup. This process was again evaluated at all three selected fan powers, and for each gust power the wing was studied at effective angles of attack of 0,5, and 10 degrees. These interactions showed that the gust acted on the wing much like an increase in angle of attack: the flow began to slowly separate from the wing until the wing was in a fully stalled state. The gust actuation process was then compared to a wing at constant angles of attack and no gust present. The vorticity strength created in both the gust and static wing cases showed many similarities, but the path the vorticity followed was quite different. It was seen that the vorticity followed a curved path for the gust-wing case while it remained 
largely straight in the static wing case. These comparisons with the static wing cases showed that quasi-static analysis of gust interactions are likely to be inaccurate.

There was some thought that a dynamic wing motion would be a better representation of the gust encounter. Therefore, a wing was pitched in the wind tunnel without a gust present to see if this motion could be compared to that of the gust-wing interaction. Even more similarity was seen between this action and the gust actuation process as the vorticity in the pitching case also had a similar curved profile in certain cases. However, there were still some discrepancies in the strength and exact path of the vorticity between the gust-wing and pitching wing interaction.

\subsection{Conclusion}

The following is a list of key conclusions that can be drawn from this research:

1. The gust generator created is a practical and useful device for studying MAVscale gusts in a wind tunnel. Gust ratios of up to 0.44 were measured at the wing location.

2. A lag was noted in the growth of vorticity for the gust-wing interaction when compared to the original growth time of the other examined flow properties measured in an empty tunnel.

3. Neither the process of the gust initially hitting a wing or a wing in a fully developed gust can be accurately simulated with a wing at constant positive angles of attack below stall. Some similarities were seen in the strength of the vorticity created in both the steady-gust and actuated-gust cases, but the path the vorticity took was visibly different, suggesting that forces estimated using this type of analysis are likely inaccurate. 
4. At angles of attack above stall, a gust-wing interaction in a steady constant gust can be modeled as a wing in a gust-less environment. This is thought to be due to the fact that for wings at initially higher angles of attack, i.e. past stall angles, the vorticity is largely generated due to this initial wing angle, not the gust-wing interaction.

5. As the gust strength decreases, the wing matches more closely to a simple static angle change for the wing. However, even at low gust strengths, the curvature of the vorticity from the leading edge of the wing is still visible. Therefore, it is shown that even in very low gust ratios this curvature would still need to be accounted for in modeling or control work.

6. A gust interaction can by simulated with a pitching motion to some high level of accuracy prior to the flow returning to the steady state solution.

\subsection{Future Work}

The designed gust generator was not without flaws, as is expected from a proofof-concept model. First, there was a large amount of turbulence created by the gust. It is thought that this turbulence was attributed to the minimal amount of flow straightening added, the flexile ducting used, and the off-the-shelf, non-experimental grade fan used for the gust. These can all be easily fixed in future designs. The second issue arose when attempting to study the gust's growth in the tunnel. It was observed that the slats controlling the gust actuation were not nearly airtight and thus some vertical component of the flow was present even when they were closed. This meant that the wing only saw a maximum flow angle change of 14 degrees instead of the theoretical 22-degree change possible. Some ideas to address this problem have been 
considered, one of which is to remove the slats entirely and have a $2^{\text {nd }}$ ducted path for the flow to follow. A powered switch would then toggle the fan to the path that either enters the tunnel when there is desire for the gust to be present, or to a secondary ducting system. The other issue mentioned earlier in this thesis is that a better interaction of the gust at the wing location could be created by moving the gust further upstream in the tunnel. However, in the current setup this would involve cutting into the aluminum base of the tunnel. The benefit of the prototype design was that it was very small and light and could be easy installed or taken down in under an hour by a single person. Once rigid ducting, a larger fan if more power is desired, and a secondary ducting loop are added, the design becomes much larger and much more difficult to move. Despite the shortcomings noted here, the device that was created and studied here did create results that outperformed previous devices in some areas. Specifically, it was able to create one of the higher gust ratios seen in the literature review, as well as create a gust that could be present around the wing for an extended period of time.

Beyond modifying the gust generator itself or running experiments with other initial wing effective angles of attack, gust speeds, or pitching rates, several other experiments with the current (or future) generator would be performed. For example, the current work lacks force data because system noise was too high and forces were too low, to get an accurate measurement. Therefore, future work should include force measurements. This either means further improving upon the current force measuring system or redesigning the gust-generator to be able to run at higher tunnel speeds where forces can be more easily measured. With force data, it would be interesting to determine the stall point of the wing in three separate cases: due to the gust interaction, 
due to the pitch, and due to static angle increases. This comparison, as well as further comparison between pitching and gust-wing interactions, would provide more information on the flow physics of gust encounters.

Much of the current fundamental fluids research community focuses their experimentation on flat plates. Flat plates are seen as easier to model and further break down the research into an even more fundamental case. Therefore, for this research to be more easily compared with other current research efforts, similar test cases should be run as presented here, but on a flat plate. This would allow greater collaboration and comparison between cases across the community.

The final area of suggested future work is for its use in the testing of MAVs. Once the scope of the problem has been analyzed with a canonical wing, the next step would be to analyze an actual MAV's interaction with this gust and record flow data from this, similar to the effort made by Kubo [55] and Shumway et al [91], but with this novel gust-generator. Thus, a final use for this test setup could be to determine if a control algorithm designed and implemented on a drone would actually mitigate the MAV's gust response in a controlled laboratory setting before taking it out for field tests. This would further the knowledge of the gust problem, not just on a fundamental level but on a system-wide level. 


\section{Appendix A: Characterization}

\section{A.1 Gust on Tunnel Off Mid and Low Power Cases}

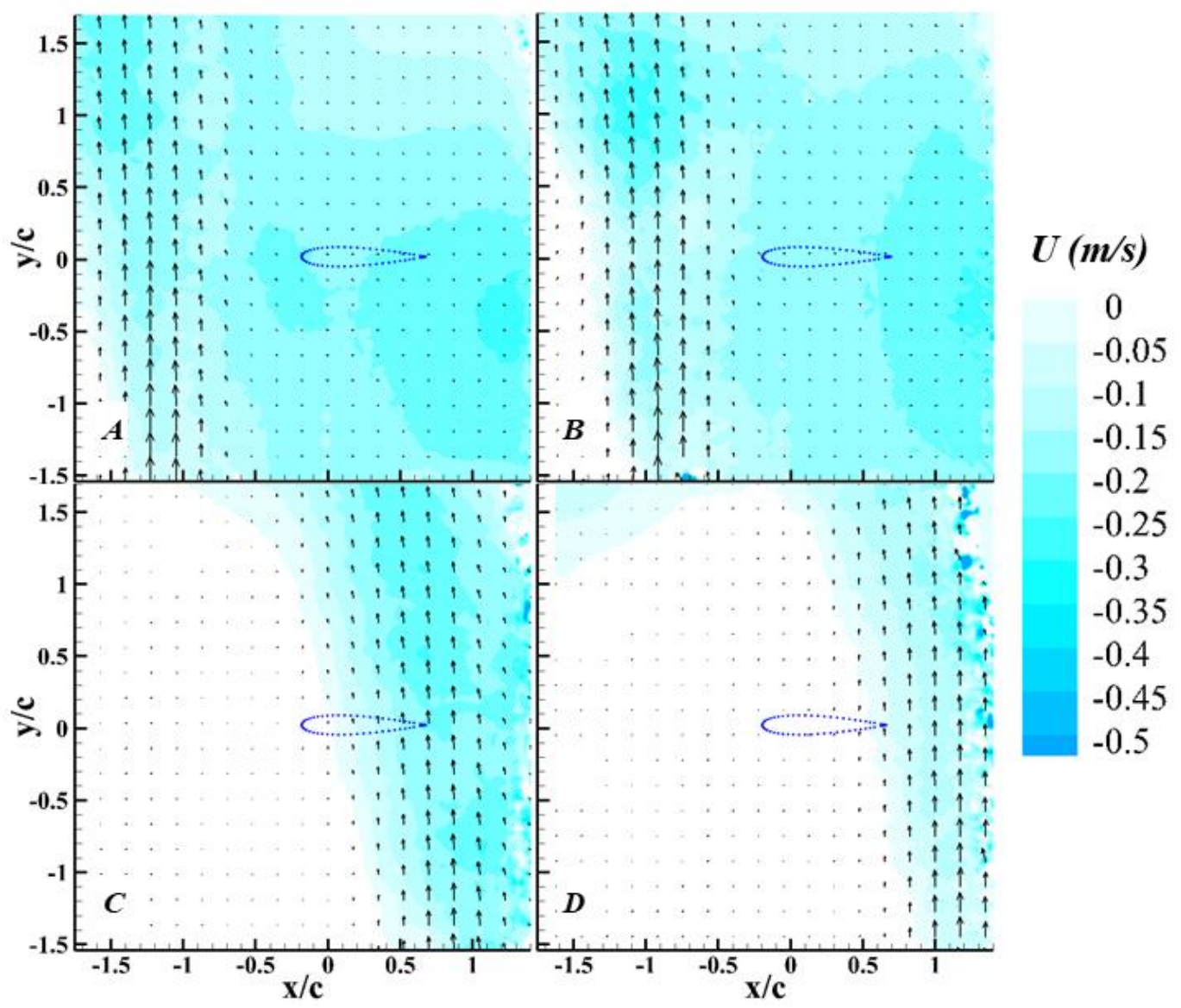

Figure 110: U velocity contour of full powered gust, no freestream.

A) Gust position 1. B) Gust position 2. C) Gust position 3. D) Gust position 4. 


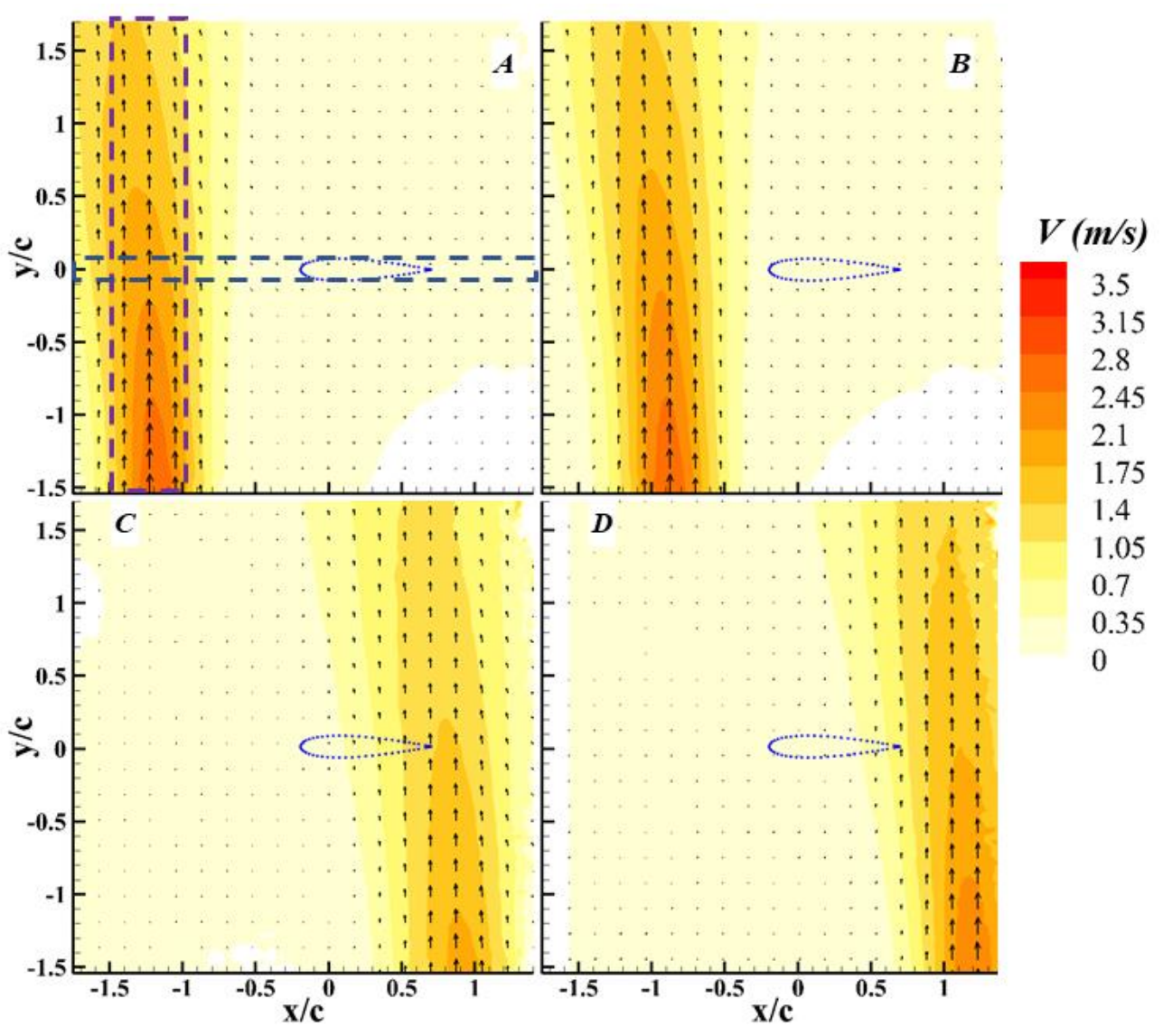

Figure 111: V velocity contours of mid power gust, no freestream. A) Gust position 1. B) Gust position 2. C) Gust position 3. D) Gust position 4. 


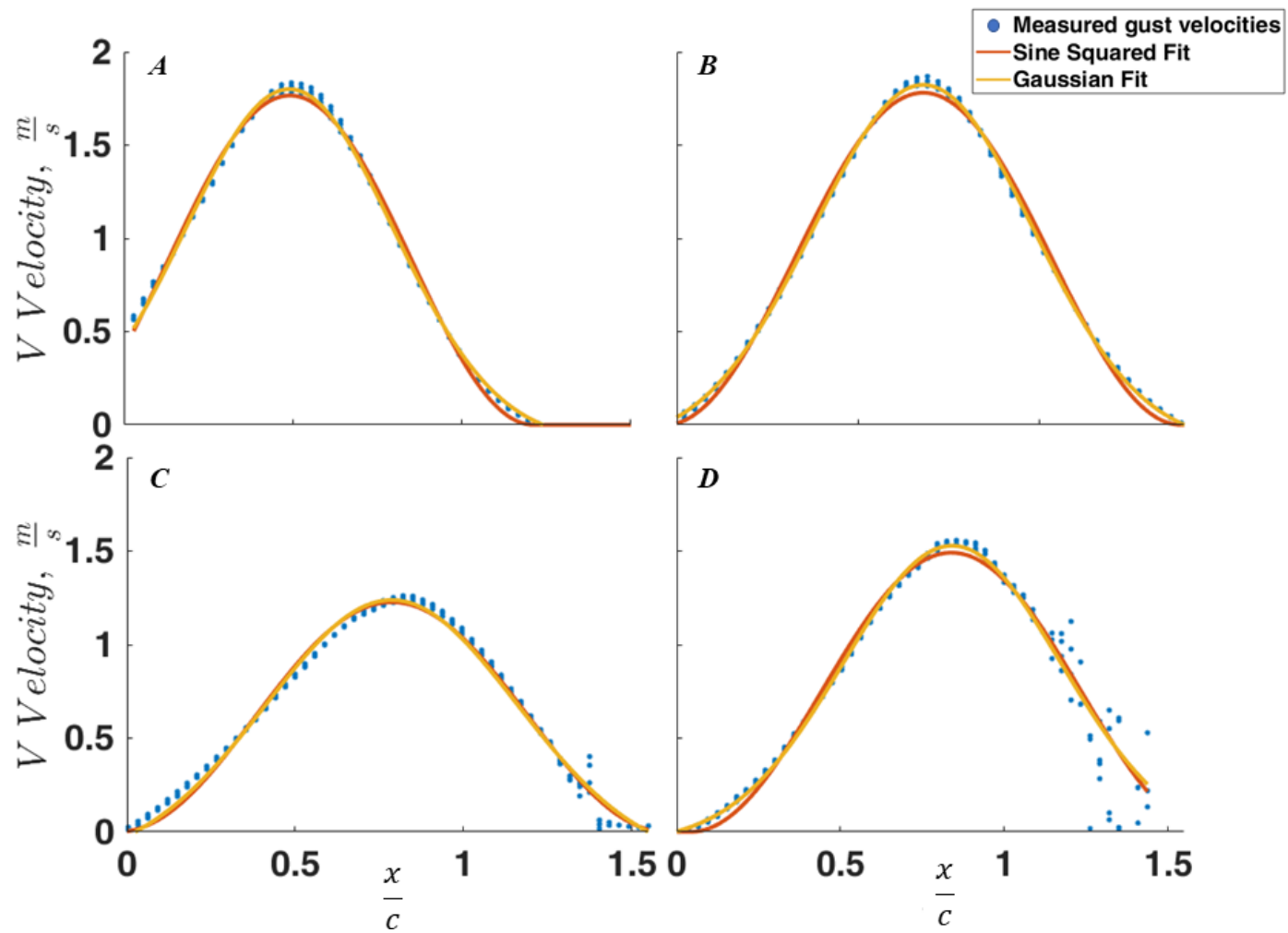

Figure 112: Mid power gust profile at wing height.

A) Gust position 1. B) Gust position 2. C) Gust position 3. D) Gust position 4. 

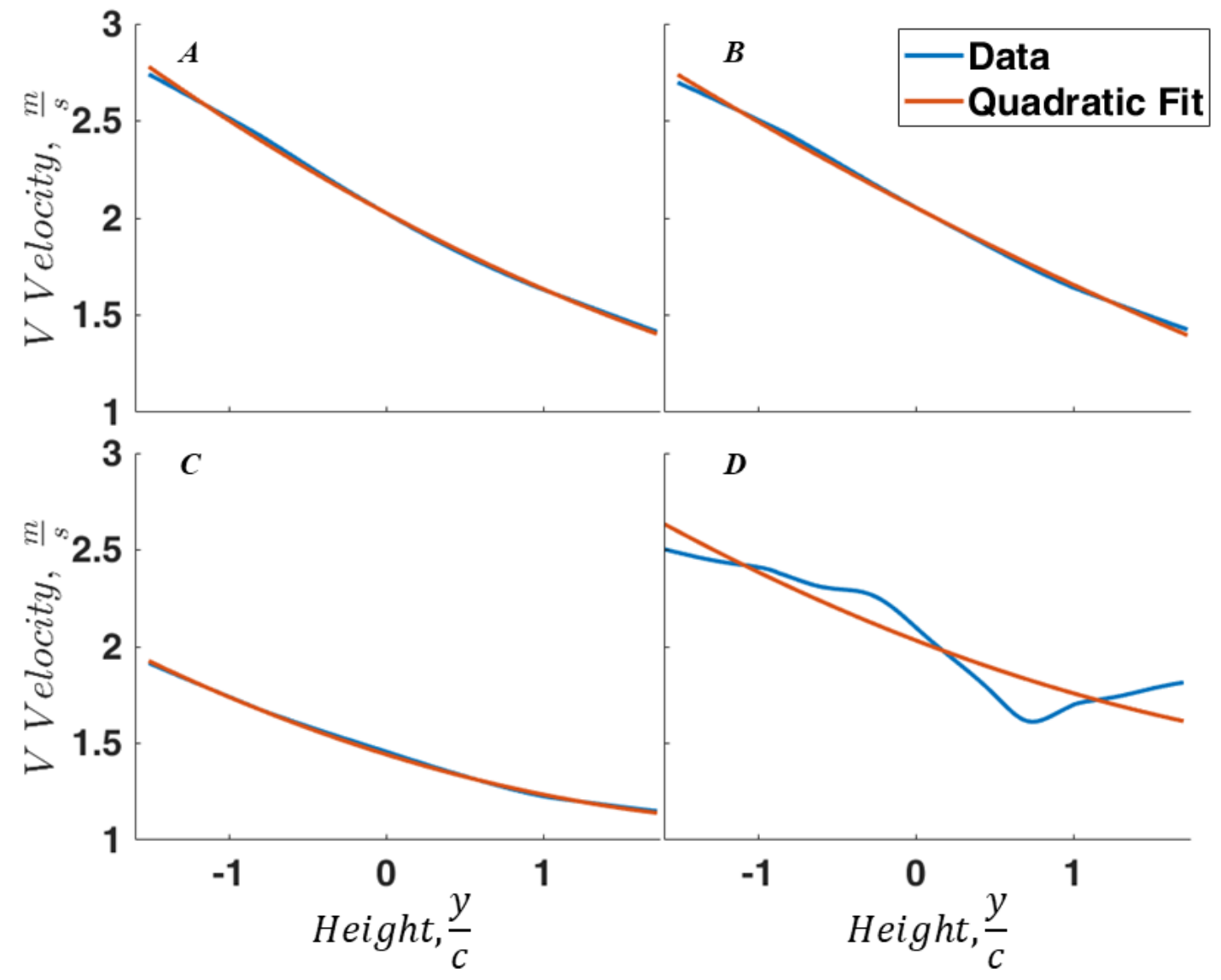

Figure 113: Mid power gust strength vs height.

A) Gust position 1. B) Gust position 2. C) Gust position 3. D) Gust position 4. 


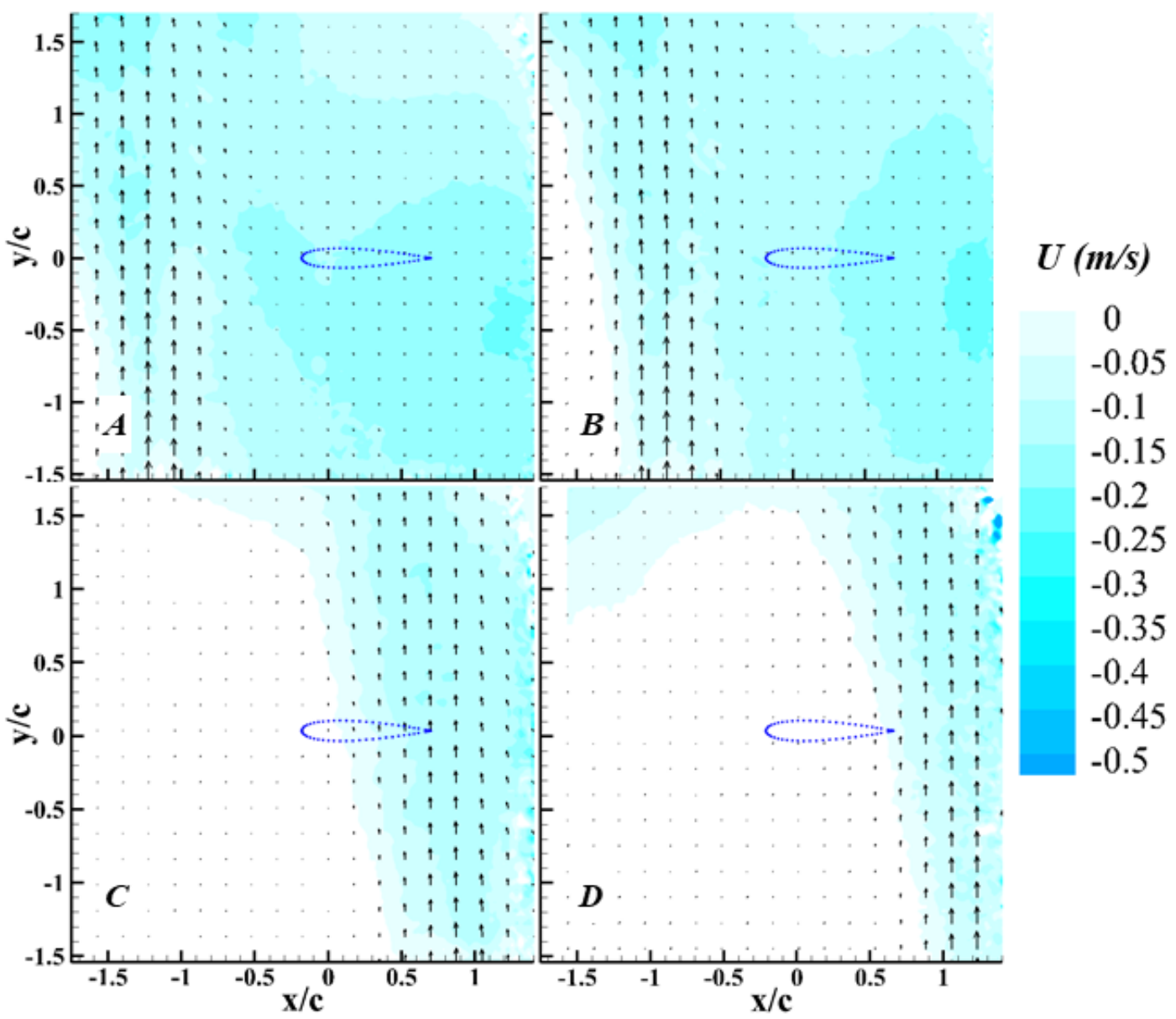

Figure 114: U velocity contour of mid powered gust, no freestream.

A) Gust position 1. B) Gust position 2. C) Gust position 3. D) Gust position 4.
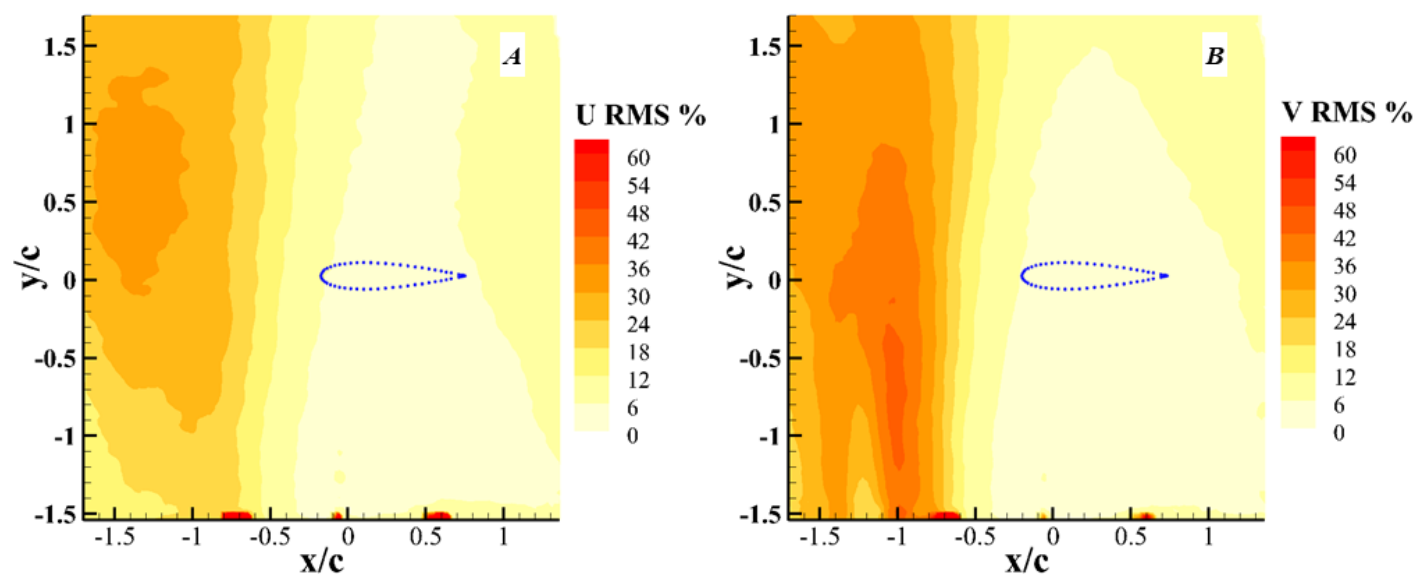

Figure 115: Mid Power U and V RMS as percentage of nominal freestream at Position 1. A) URMS percentage 1, B) VRMS percentage. 


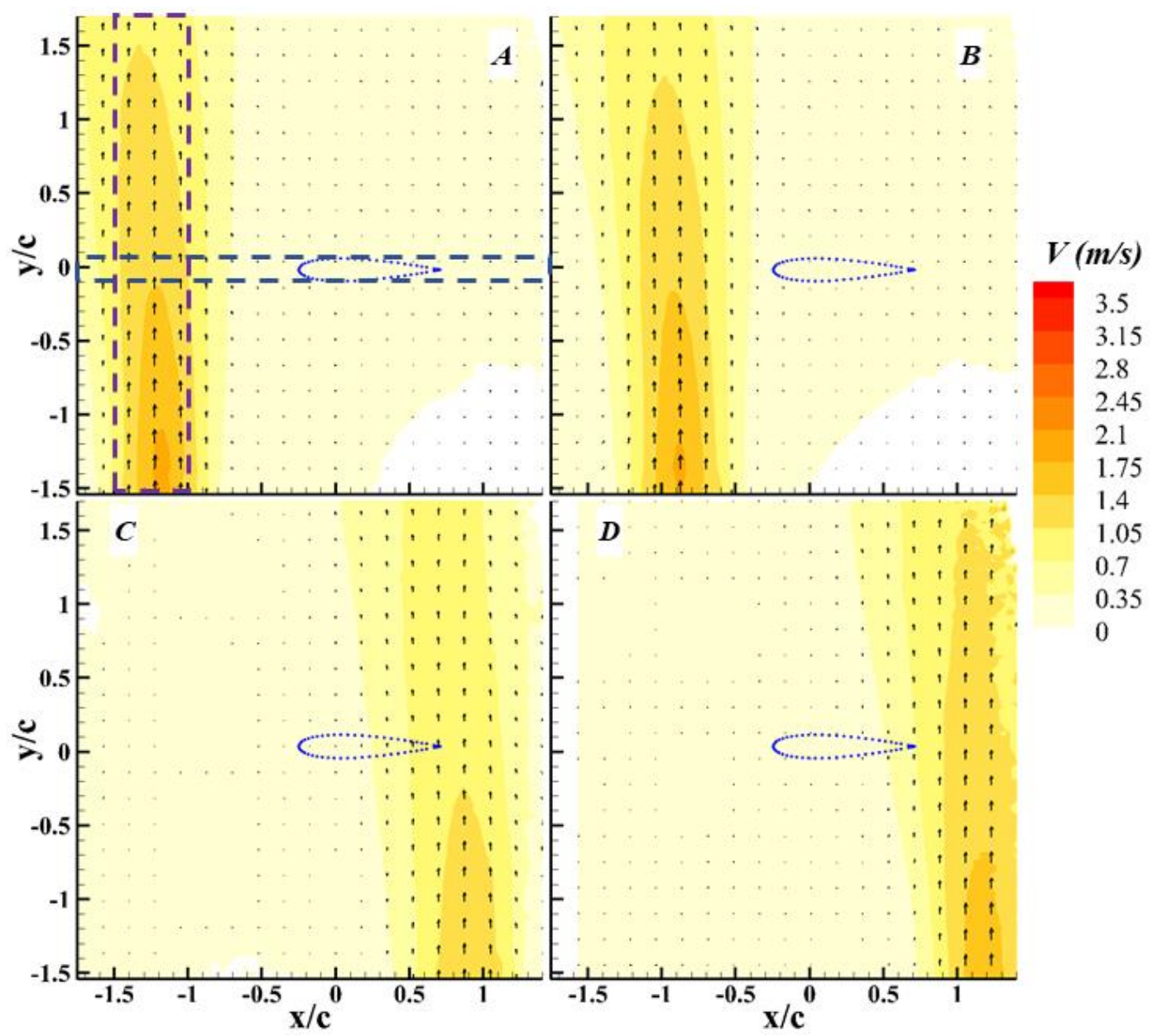

Figure 116: V velocity contours of low power gust, no freestream.

A) Gust position 1. B) Gust position 2. C) Gust position 3. D) Gust position 4. 


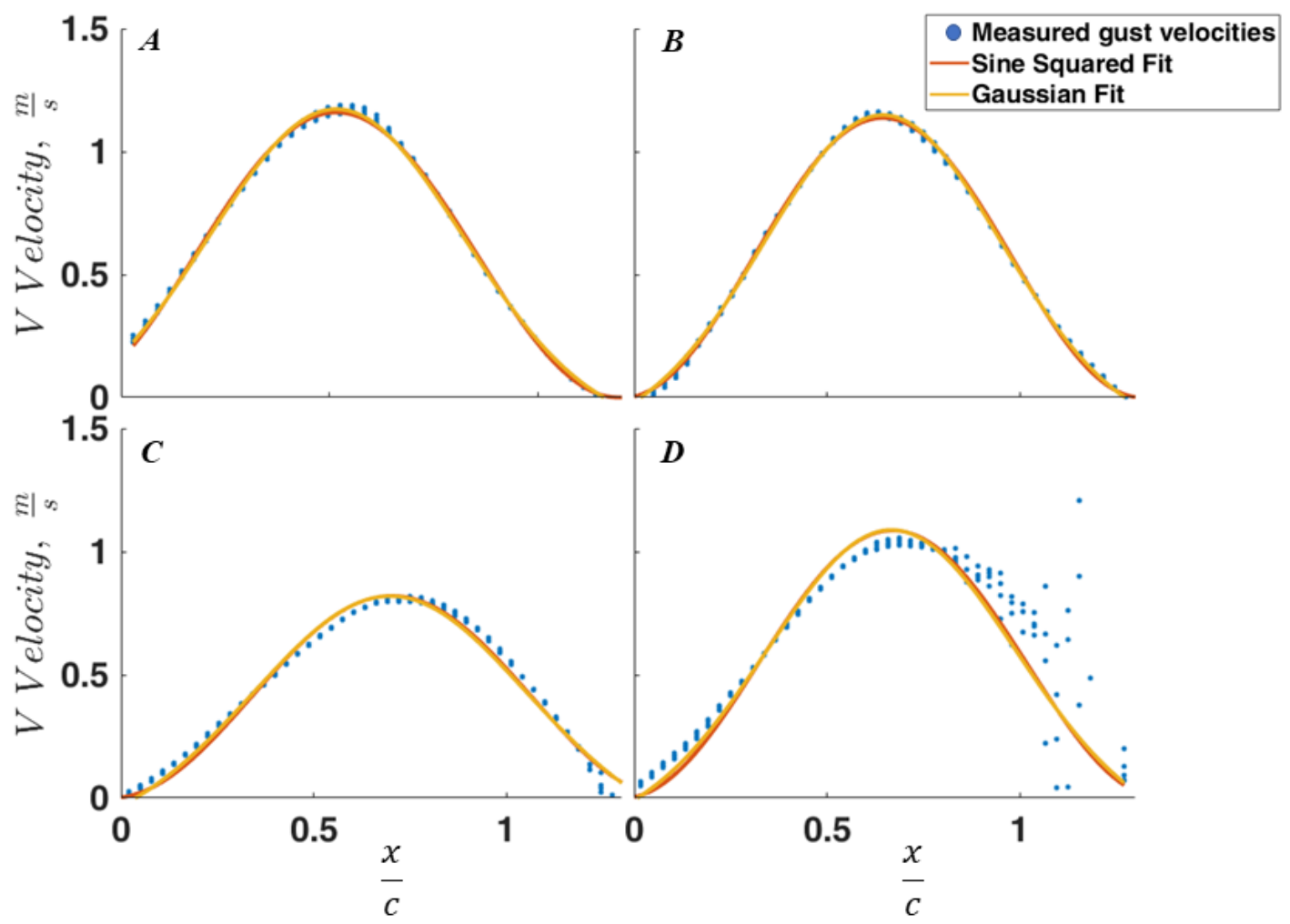

Figure 117: Low power gust profile at wing height.

A) Gust position 1. B) Gust position 2. C) Gust position 3. D) Gust position 4. 


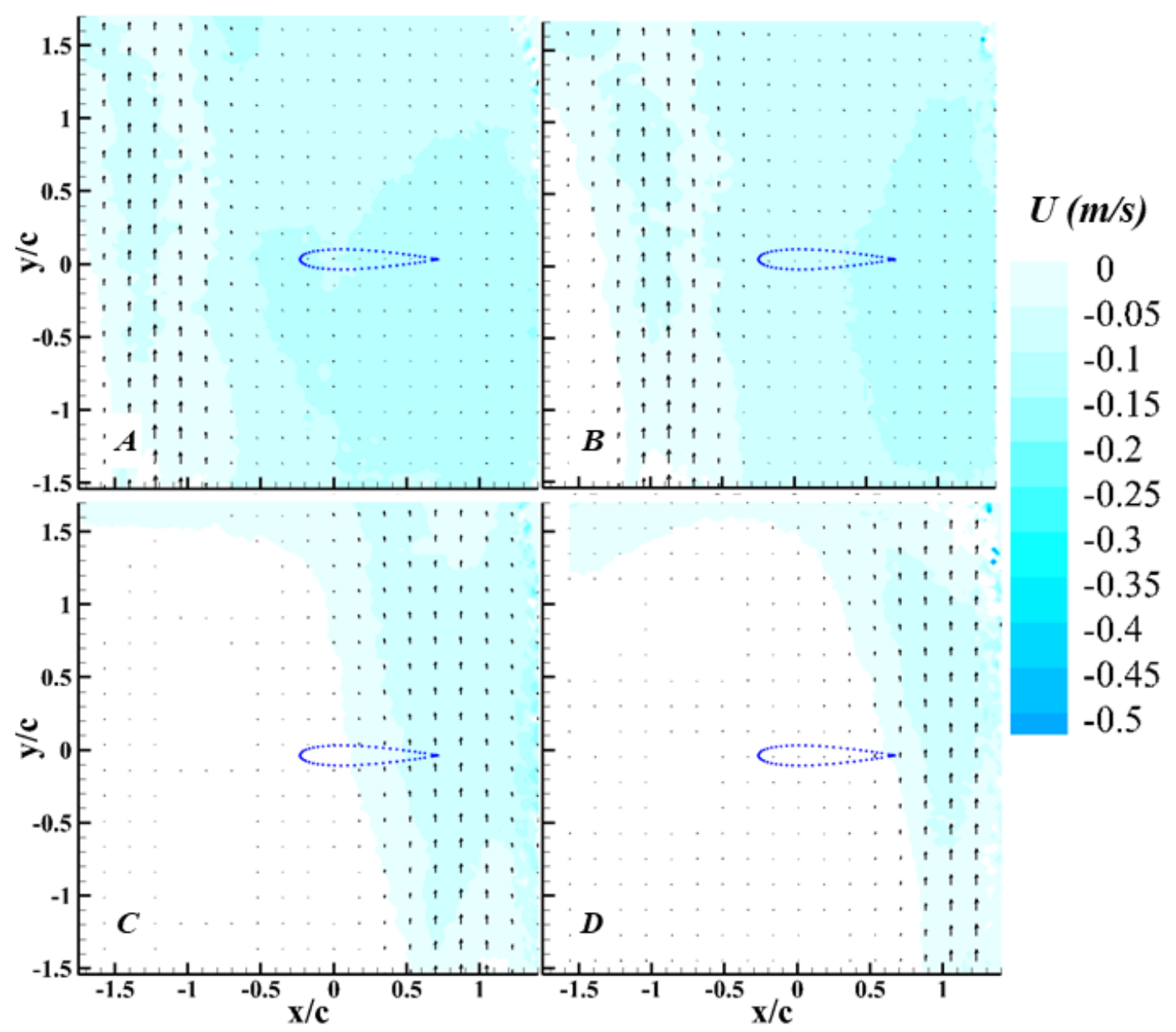

Figure 118: U velocity contour of low powered gust, no freestream.

A) Gust position 1. B) Gust position 2. C) Gust position 3. D) Gust position 4.

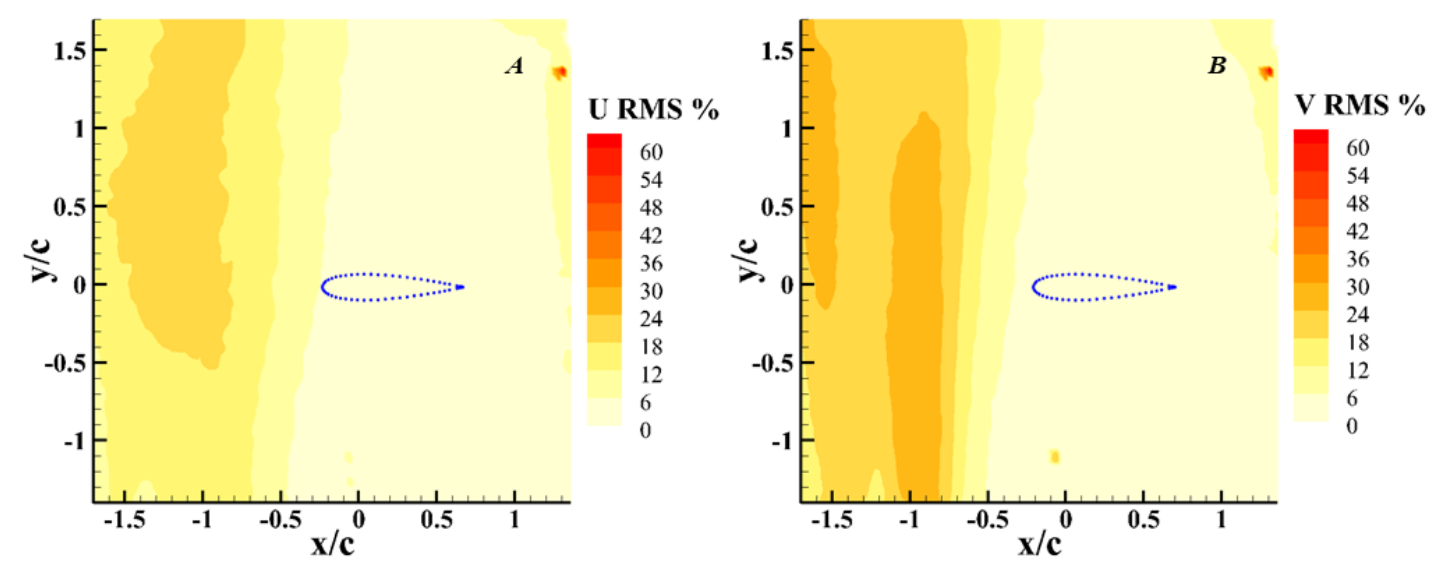

Figure 119: Low power $U$ and V RMS as a percentage of nominal freestream flow at Position 1.

A) $U R M S$ percentage, B) VRMS percentage. 
Some notes from analyzing these images: As before, the decrease in vertical (V) velocity between Positions 1 and 2, and Positions 3 and 4 were seen. Compared to the full power cases there was a clear, and easily predicted, drop in gust velocity for both the mid and low power cases. It was found that at Position 1, the mid power case had a vertical velocity at the wing height of $2.00 \mathrm{~m} / \mathrm{s}$ and a speed leaving the generator of $3.57 \mathrm{~m} / \mathrm{s}$. Similarly, the low power case had a speed of $1.35 \mathrm{~m} / \mathrm{s}$ at the wing height and a speed leaving the generator of $2.31 \mathrm{~m} / \mathrm{s}$. The width of the low power case was 1.35 chords and the mid power case was 1.42 chords wide. The results from all three powers show that the rheostat itself creates a nearly linear change in gust power based on dial position.

It was also found that the gust generator produced lower fluctuations in velocity at lower power settings. RMS values inside the gust at the low power case were about $24 \%$ of the mean freestream when present. The RMS in this mid power case at the center of the gust reached about $42 \%$. This is still a bit high, but much lower than the $65 \%$ seen in the full power case. Ideas on how to further reduce this turbulence are currently being examined and will be further discussed in the future work section of this report. The same 4.2-degree angle of the gust nozzle was present in for both the low and mid power cases, but due to their slower nozzle velocities, the horizontal (U) velocity in the flow also decreased. 


\section{A.2 Gust on Tunnel on Mid and Low Power Cases}

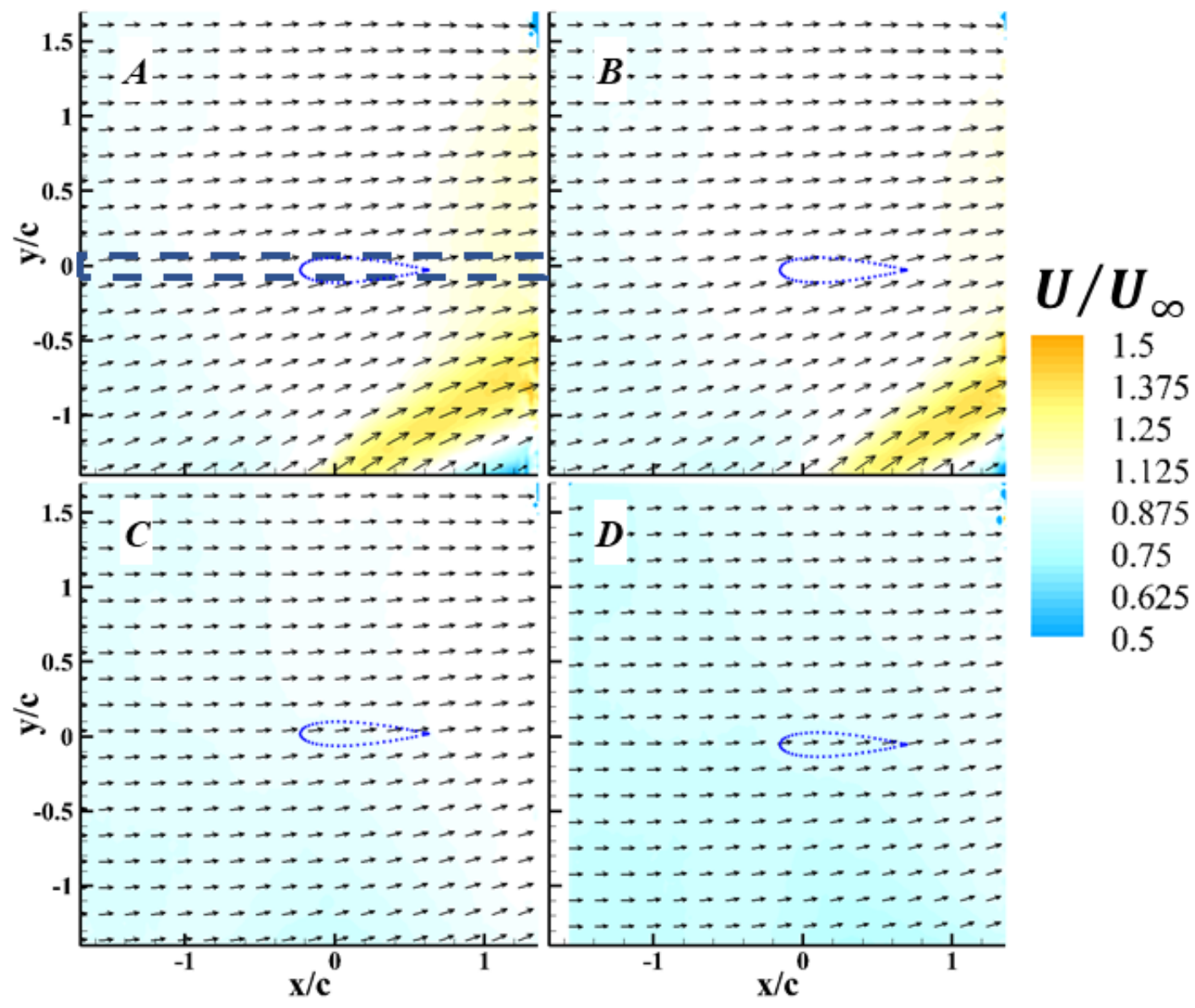

Figure 120: Mid power gust with freestream displaying contours of $U / U_{\infty}$.

A) Gust position 1. B) Gust position 2. C) Gust position 3. D) Gust position 4. 


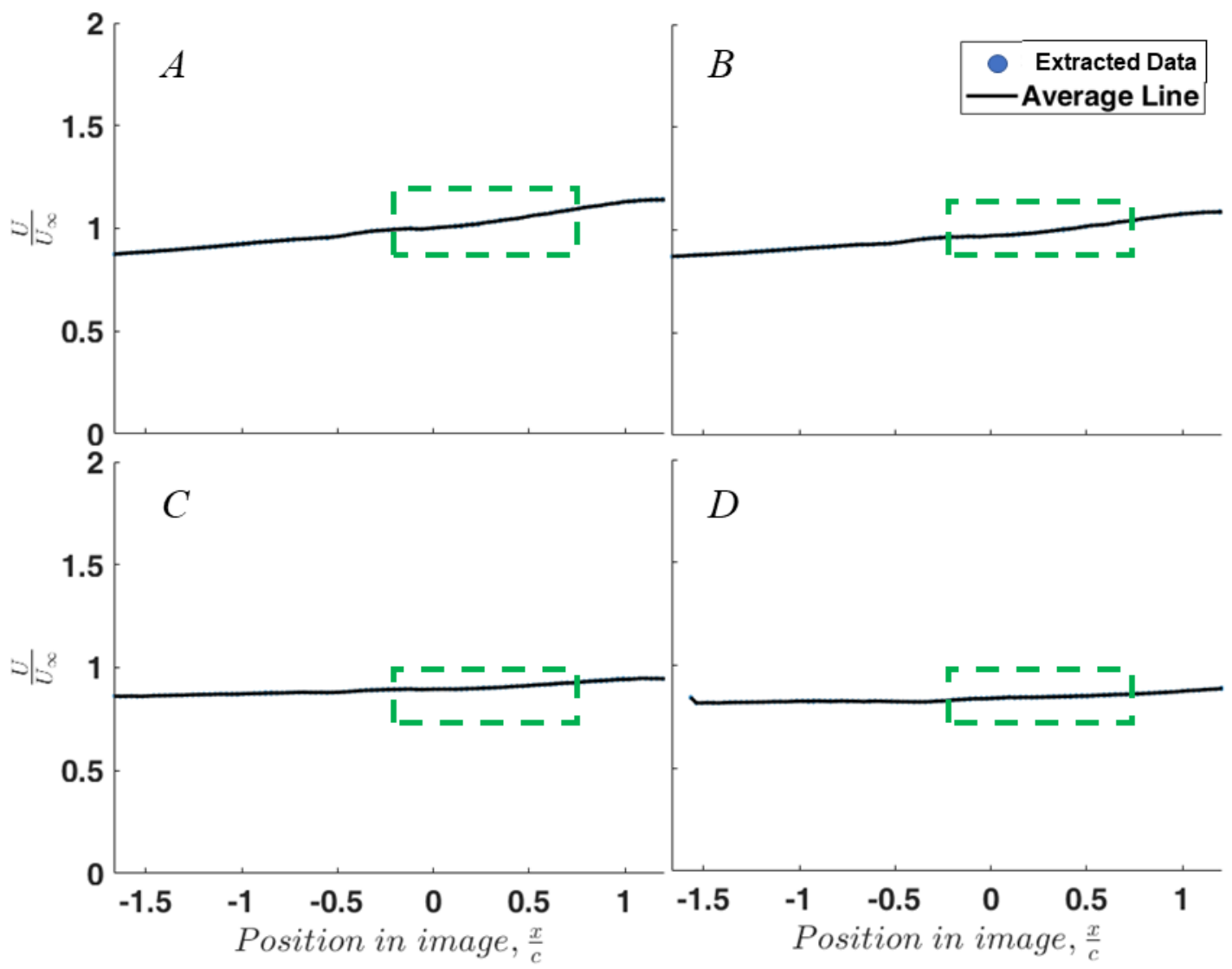

Figure 121: Mid power $U / U_{\infty}$ values at wing height.

A) Gust position 1. B) Gust position 2. C) Gust position 3. D) Gust position 4. 


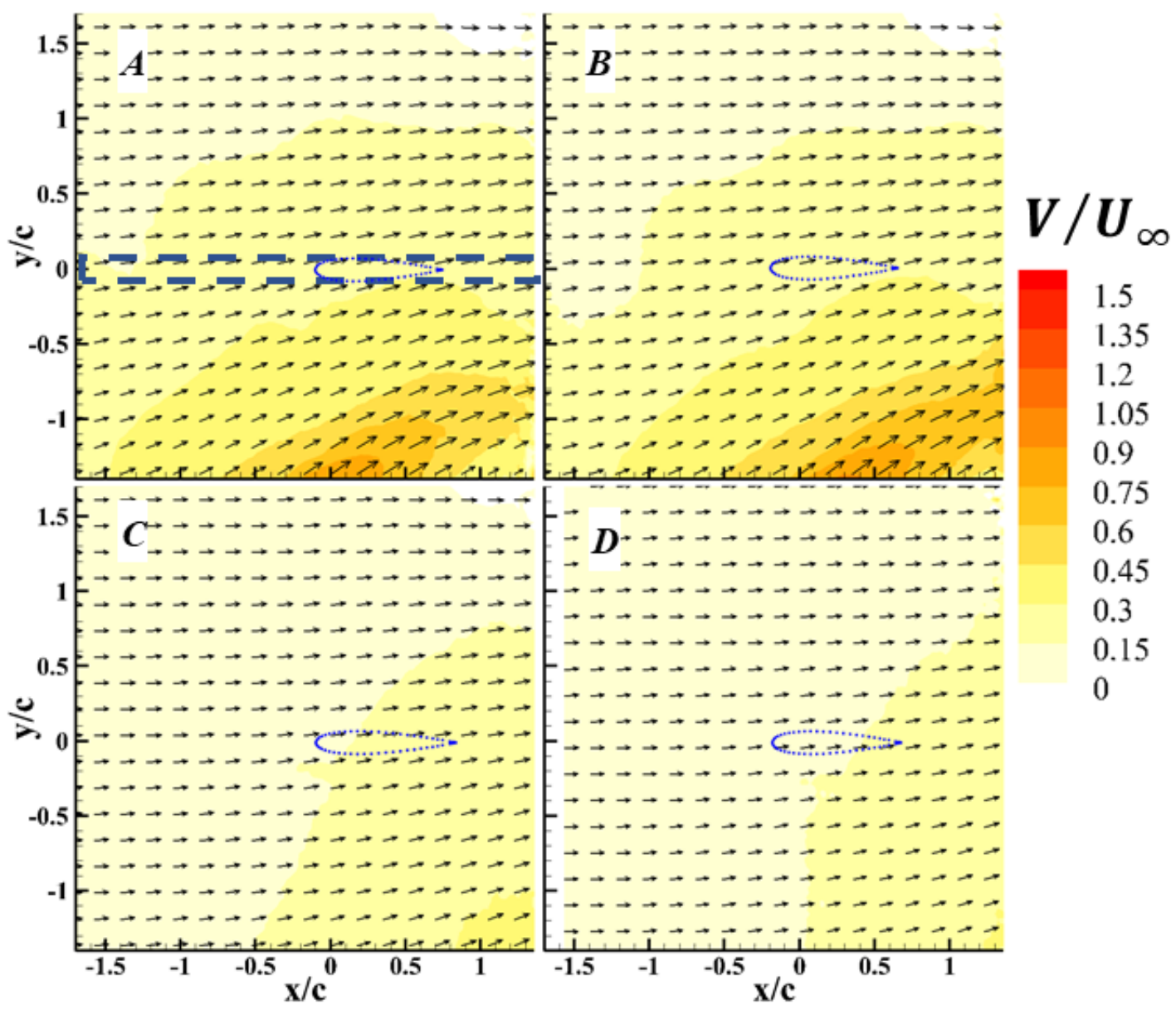

Figure 122: Mid power gust ratio contour with freestream.

A) Gust position 1. B) Gust position 2. C) Gust position 3. D) Gust position 4. 


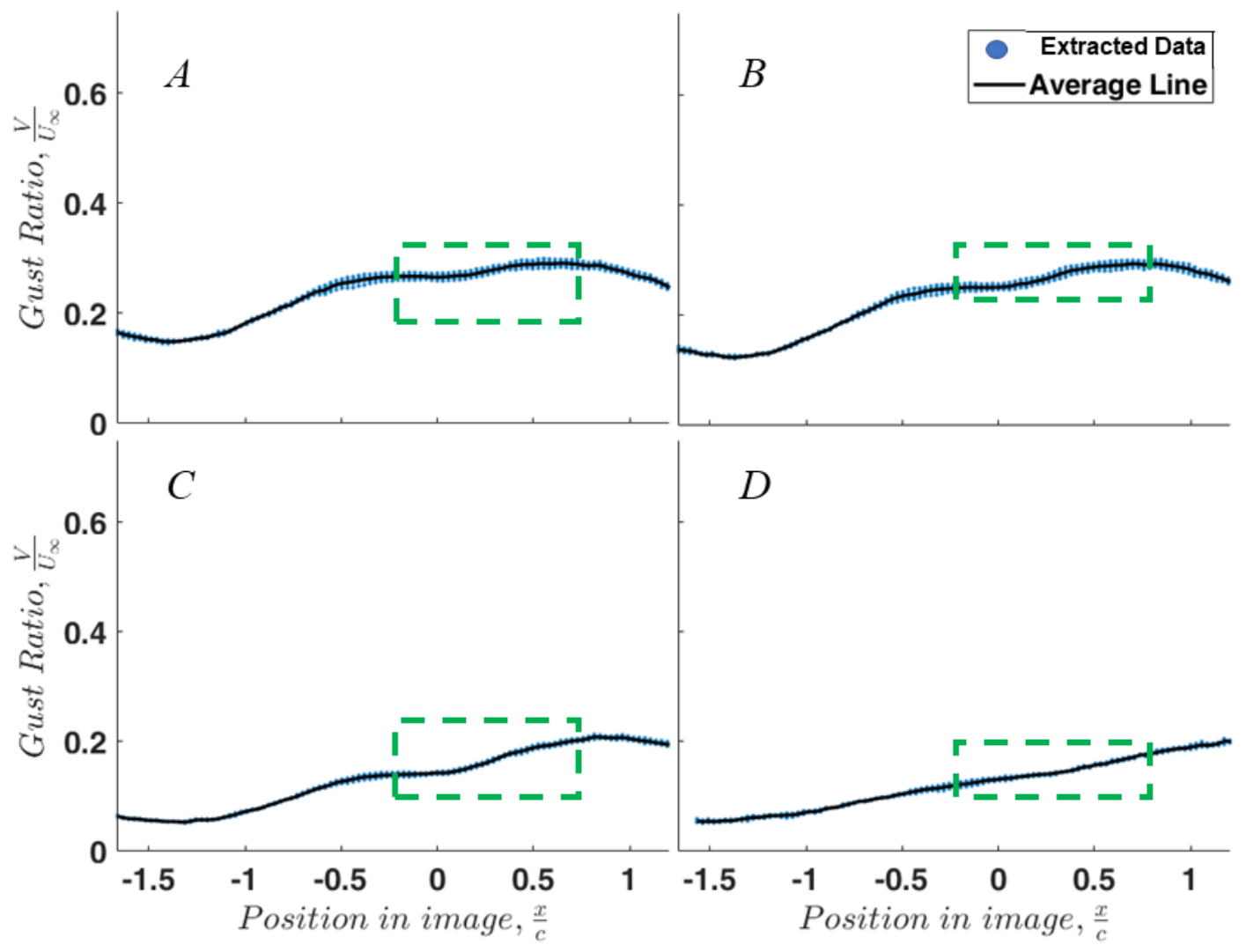

Figure 123: Mid power gust ratio values at wing height.

A) Gust position 1. B) Gust position 2. C) Gust position 3. D) Gust position 4. 


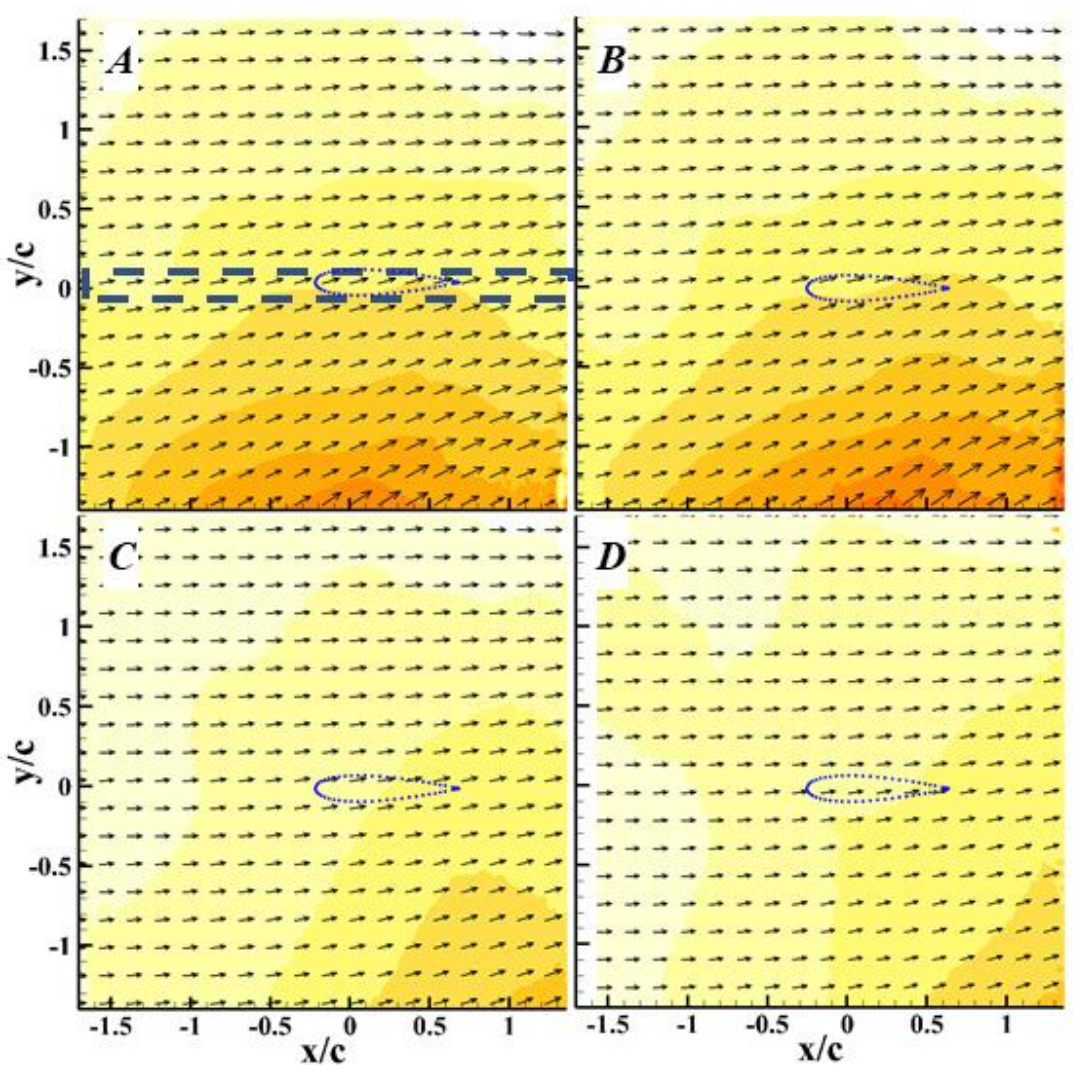

\section{Flow Angle}

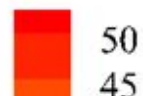

45

40

35

30

25

20

15

10

5

0

Figure 124: Mid power flow angle contours with freestream.

A) Gust position 1. B) Gust position 2. C) Gust position 3. D) Gust position 4. 


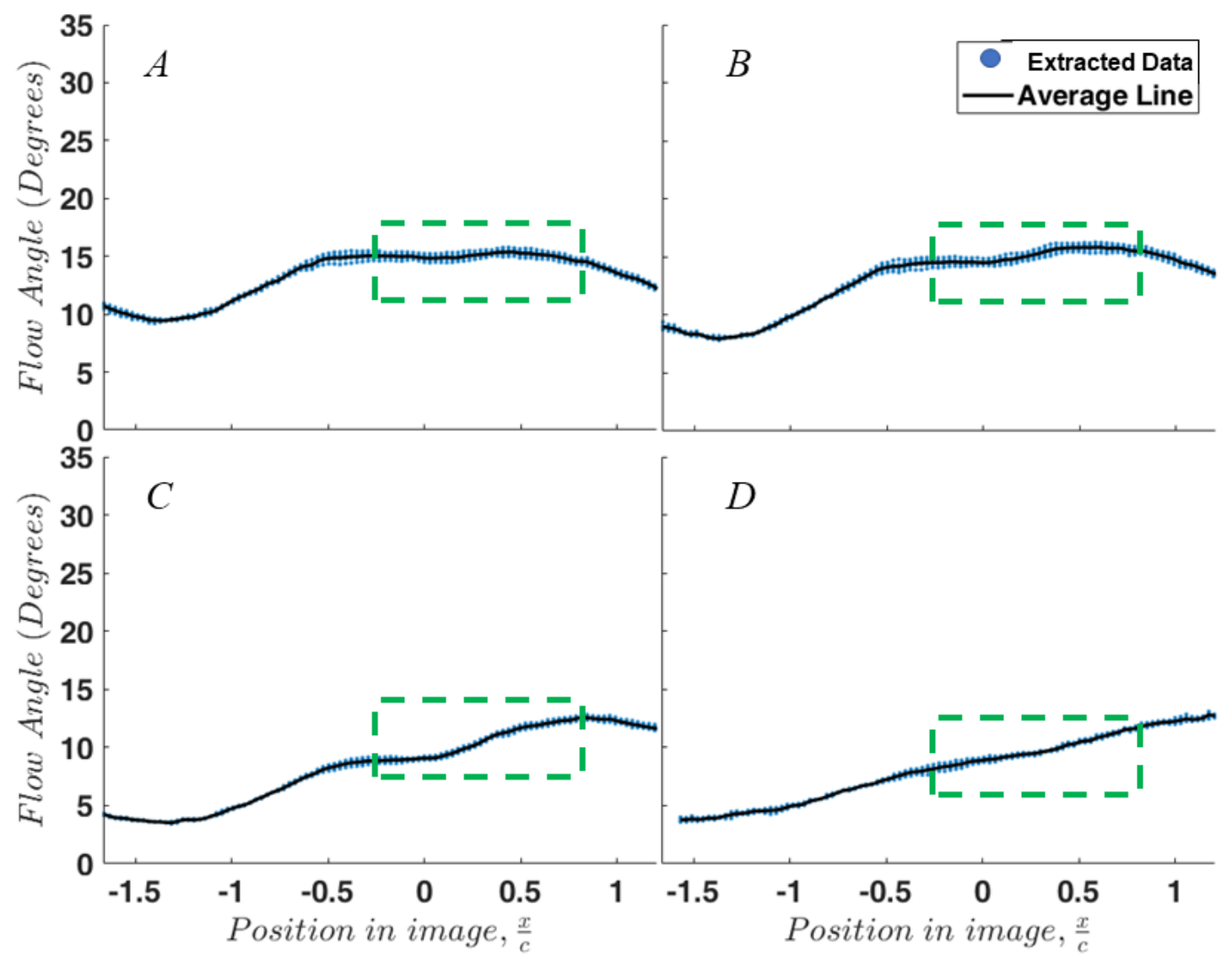

Figure 125: Mid power flow angle values at wing height.

A) Gust position 1. B) Gust position 2. C) Gust position 3. D) Gust position 4.
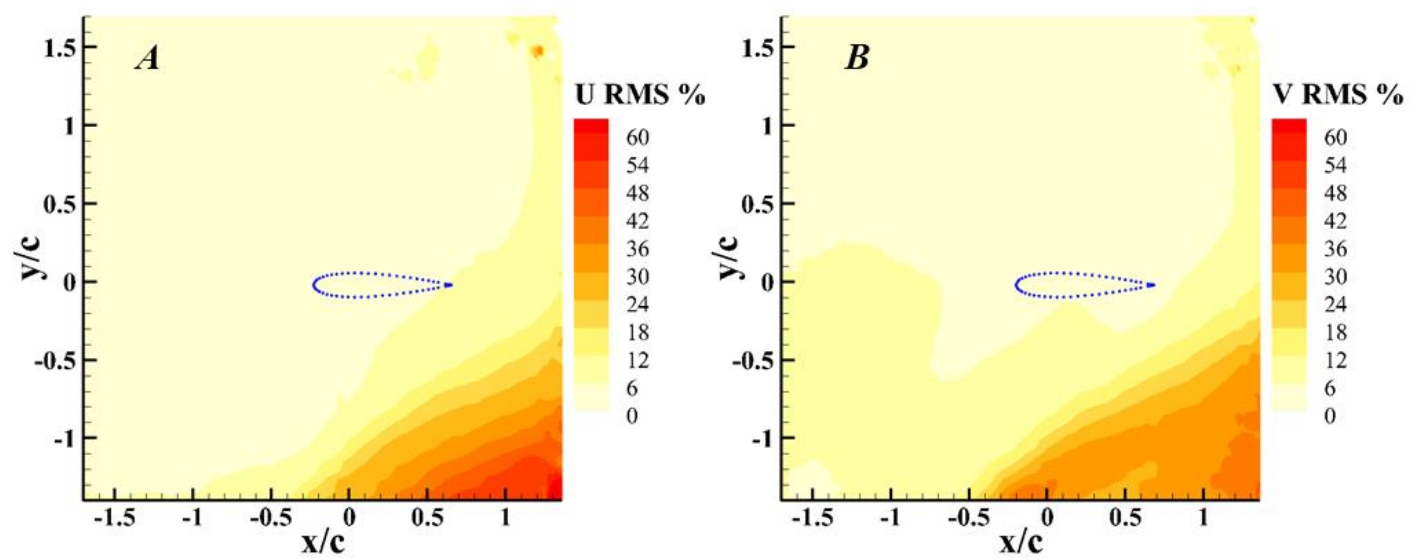

Figure 126: Mid power $U$ and V RMS as a percentage of nominal freestream flow at Position 1. A) U RMS percentage, B) VRMS percentage. 


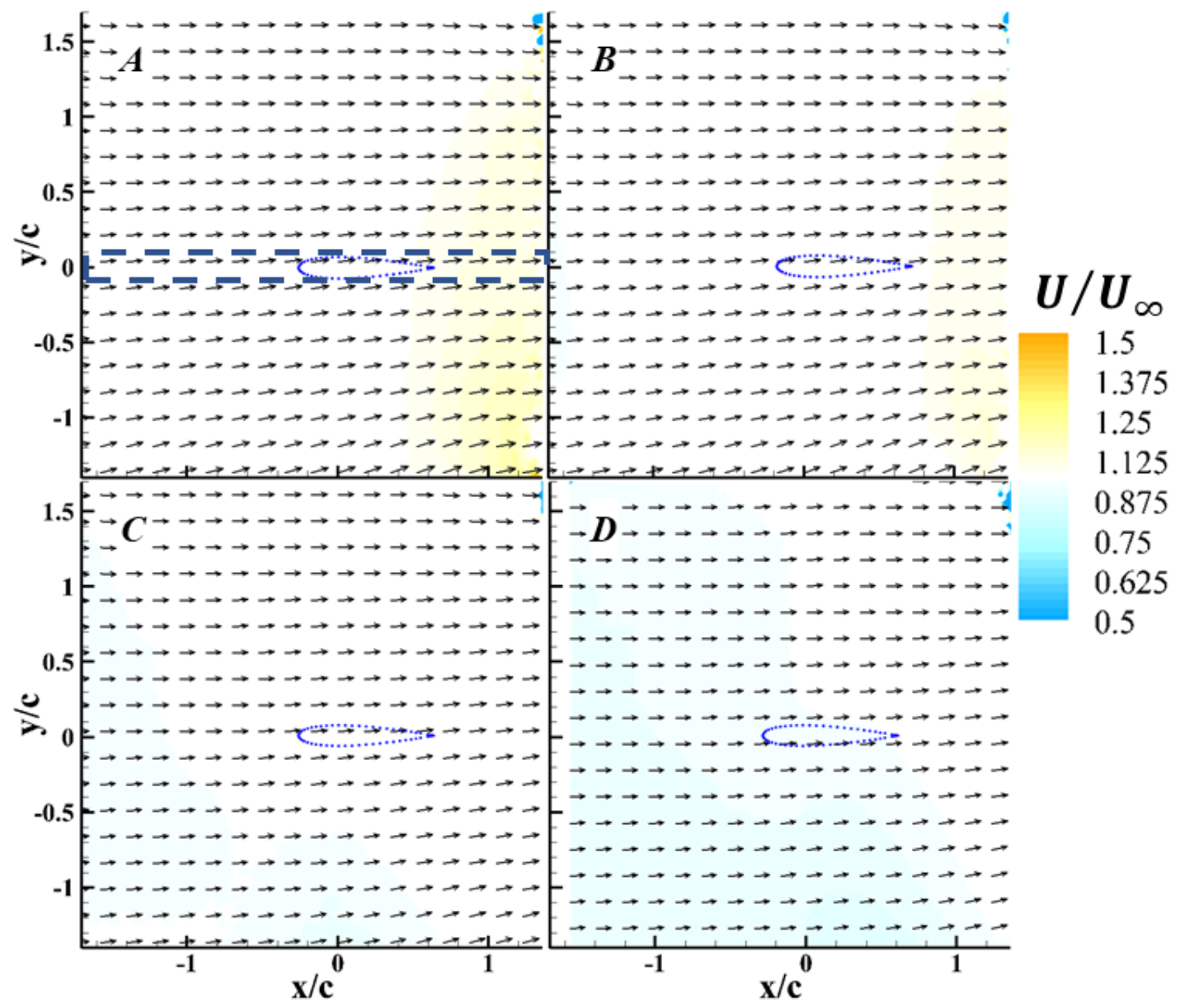

Figure 127: Low power $U / U_{\infty}$ contour with freestream.

A) Gust position 1. B) Gust position 2. C) Gust position 3. D) Gust position 4. 


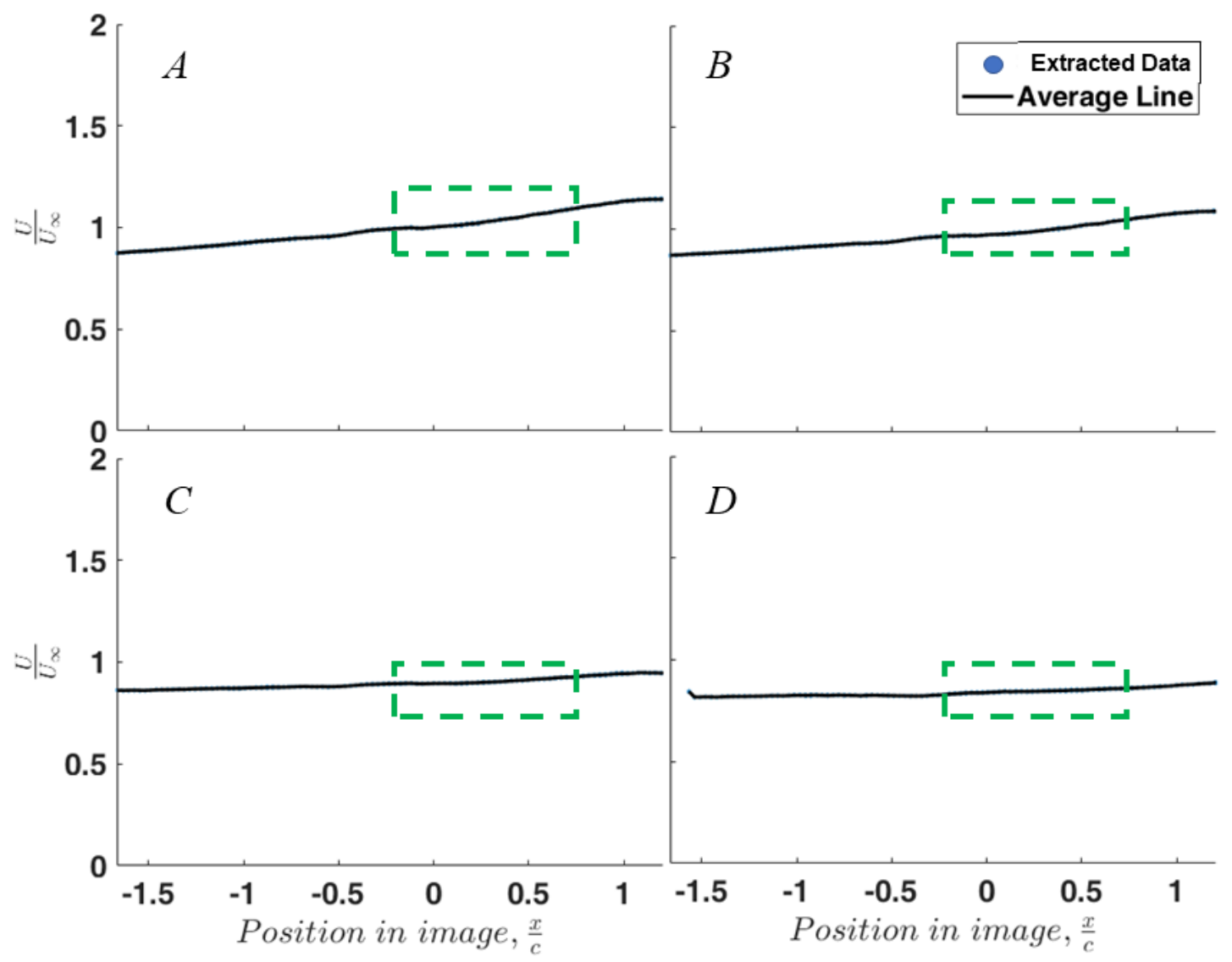

Figure 128: Low power $U / U_{\infty}$ values at wing height.

A) Gust position 1. B) Gust position 2. C) Gust position 3. D) Gust position 4. 


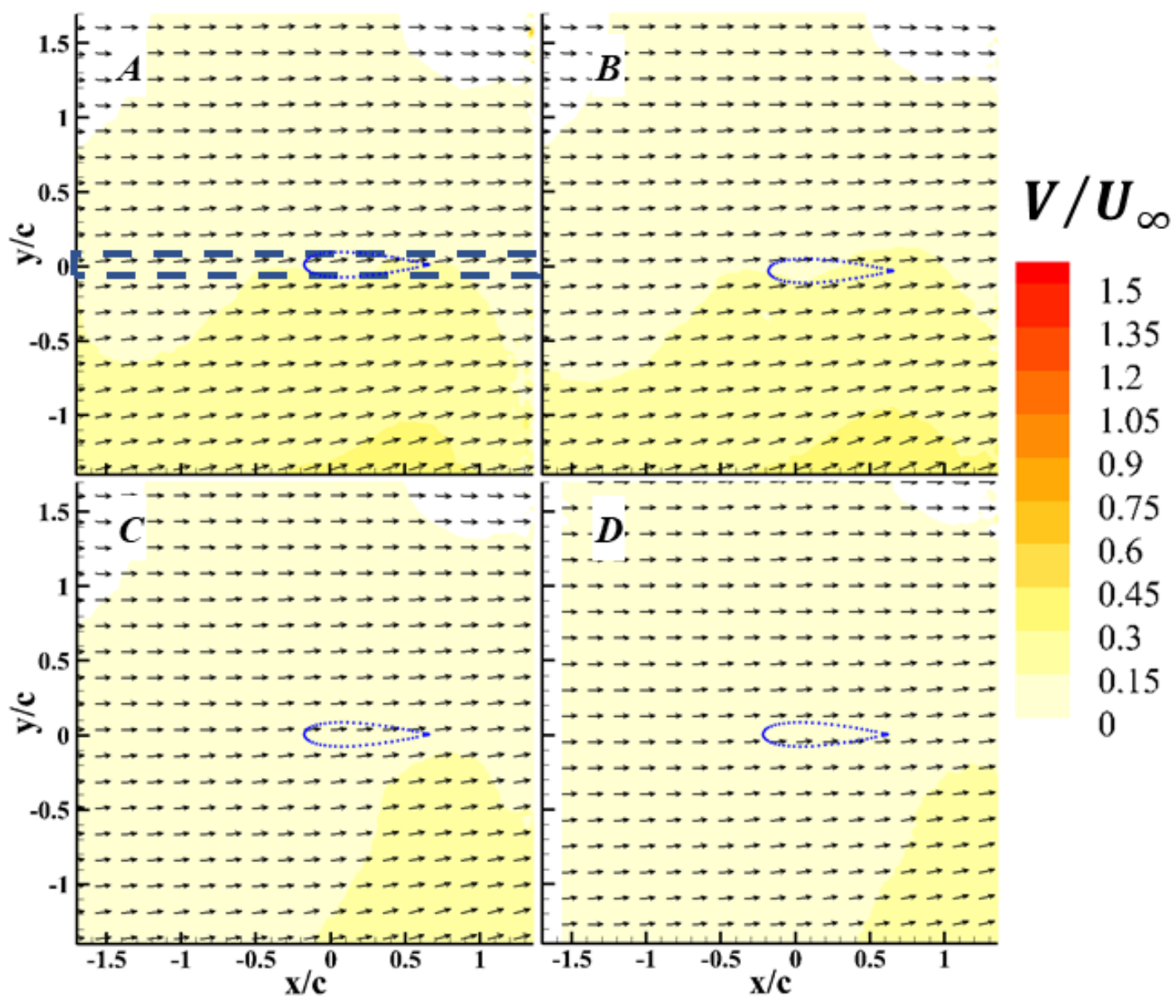

Figure 129: Low power gust ratio contour with freestream.

A) Gust position 1. B) Gust position 2. C) Gust position 3. D) Gust position 4. 


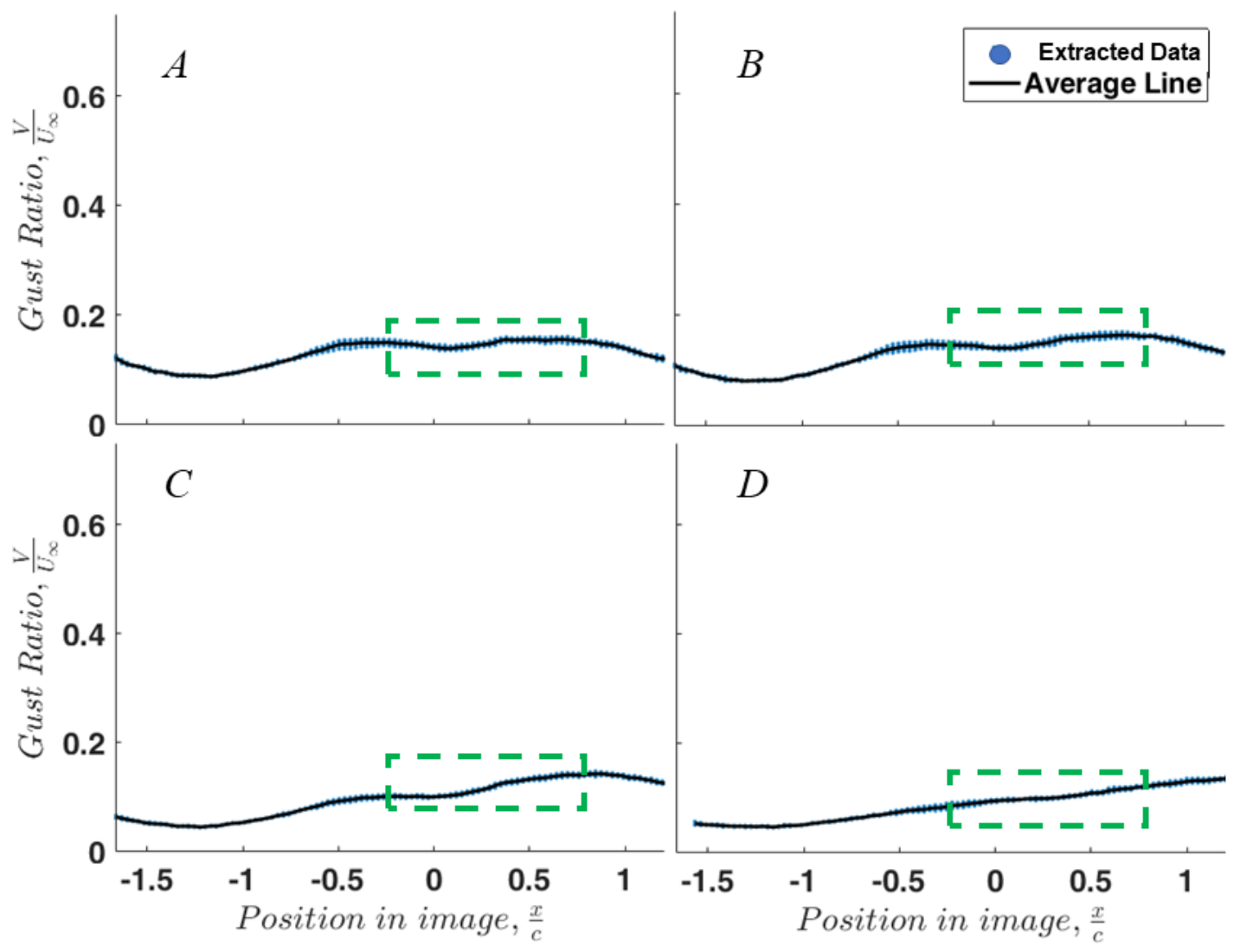

Figure 130: Gust ratio values at wing height.

A) Gust position 1. B) Gust position 2. C) Gust position 3. D) Gust position 4. 


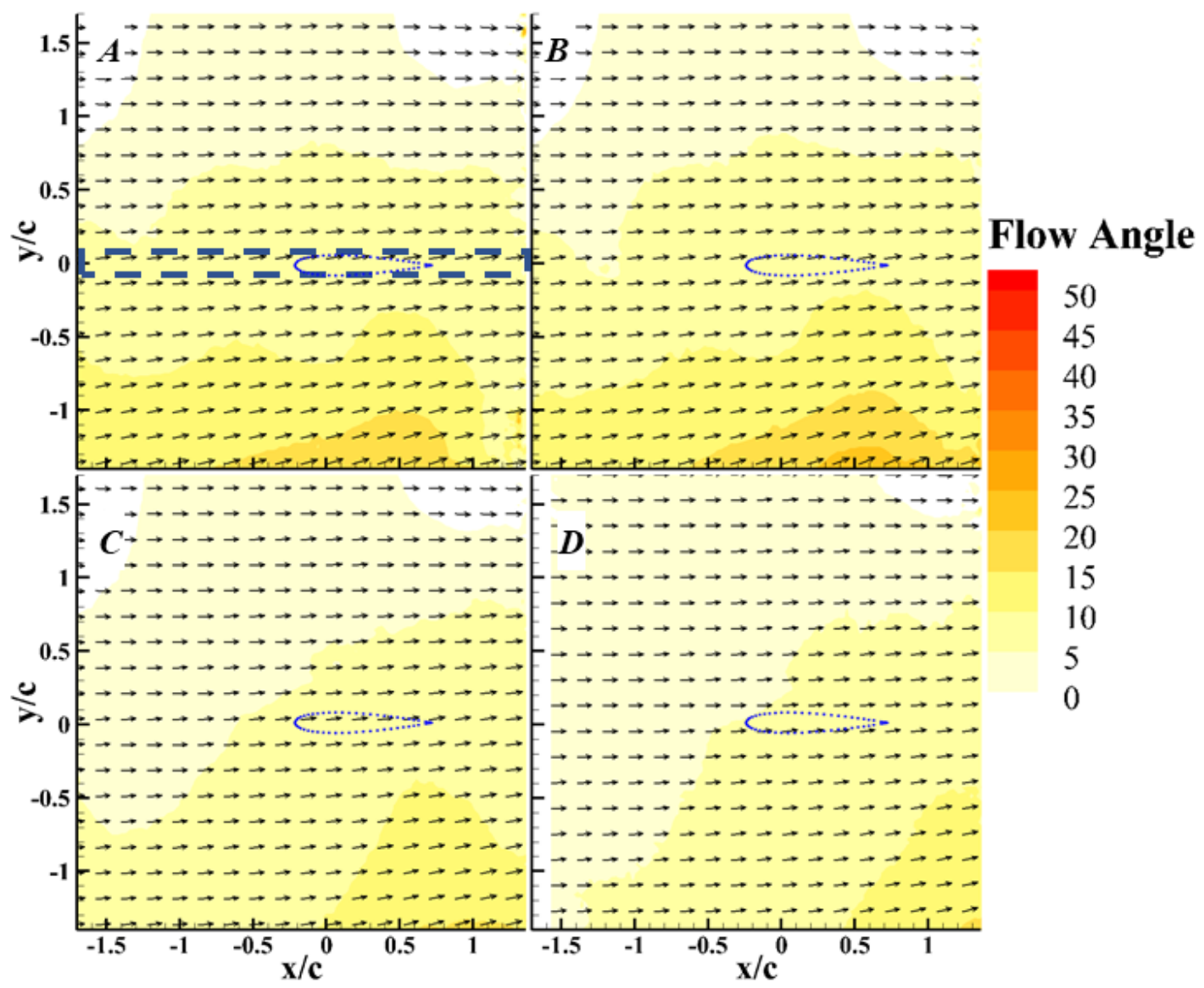

Figure 131: Low power flow angle contour with freestream. A) Gust position 1. B) Gust position 2. C) Gust position 3. D) Gust position 4. 


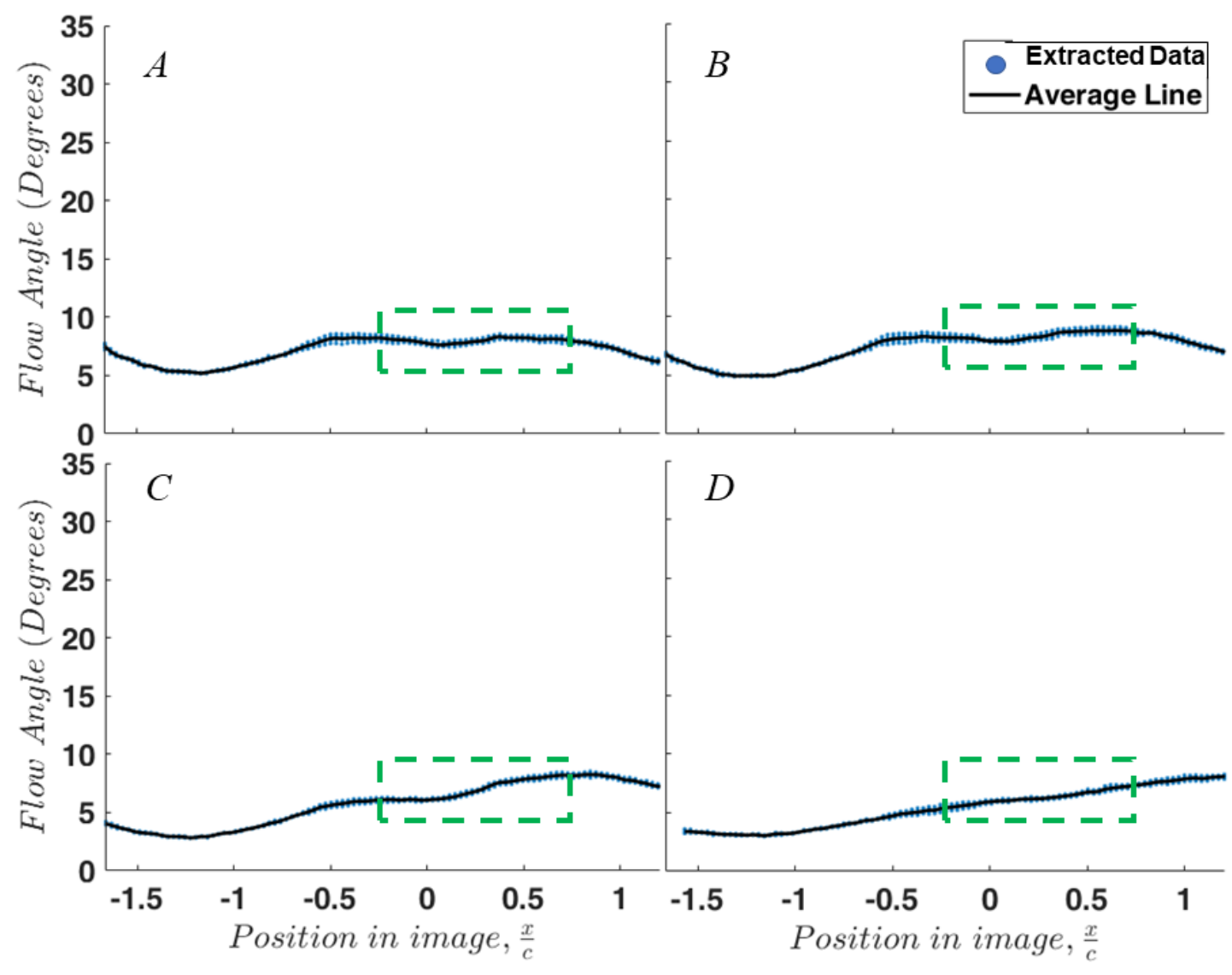

Figure 132: Gust ratio values at wing height.

A) Gust position 1. B) Gust position 2. C) Gust position 3. D) Gust position 4.

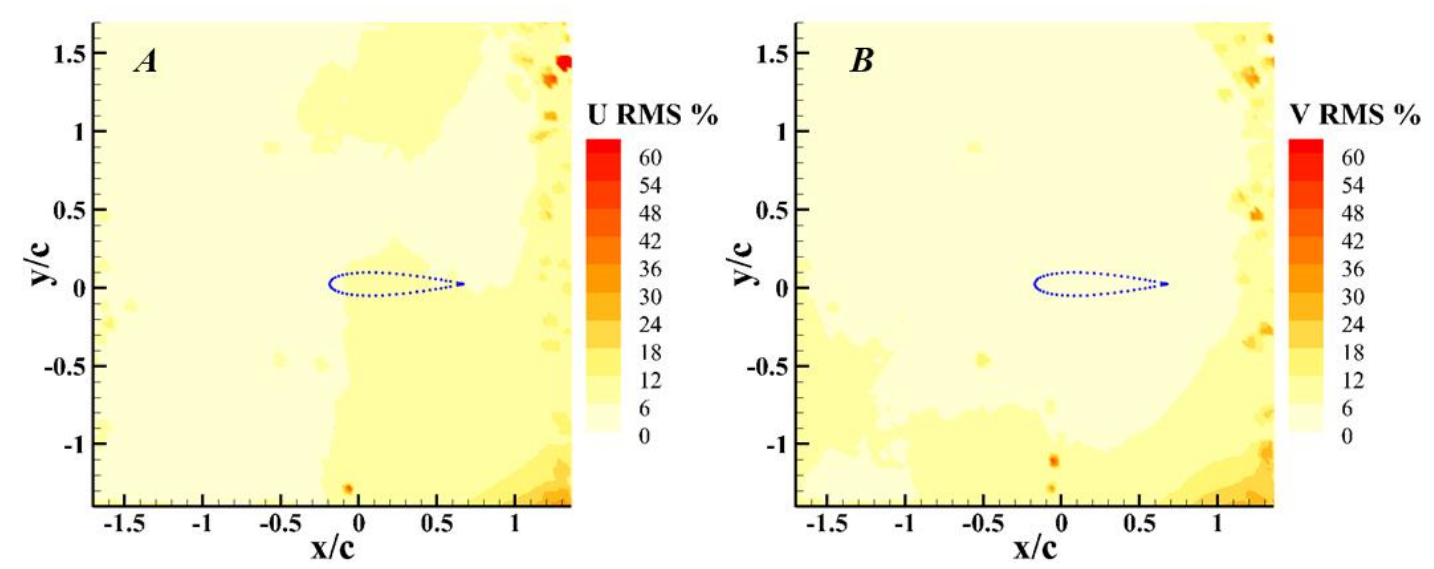

Figure 133: Low power $U$ and V RMS as percentage of nominal freestream at Position 1. A) URMS percentage $1, B) V R M S$ percentage. 


\section{Appendix B: Supplemental Results}

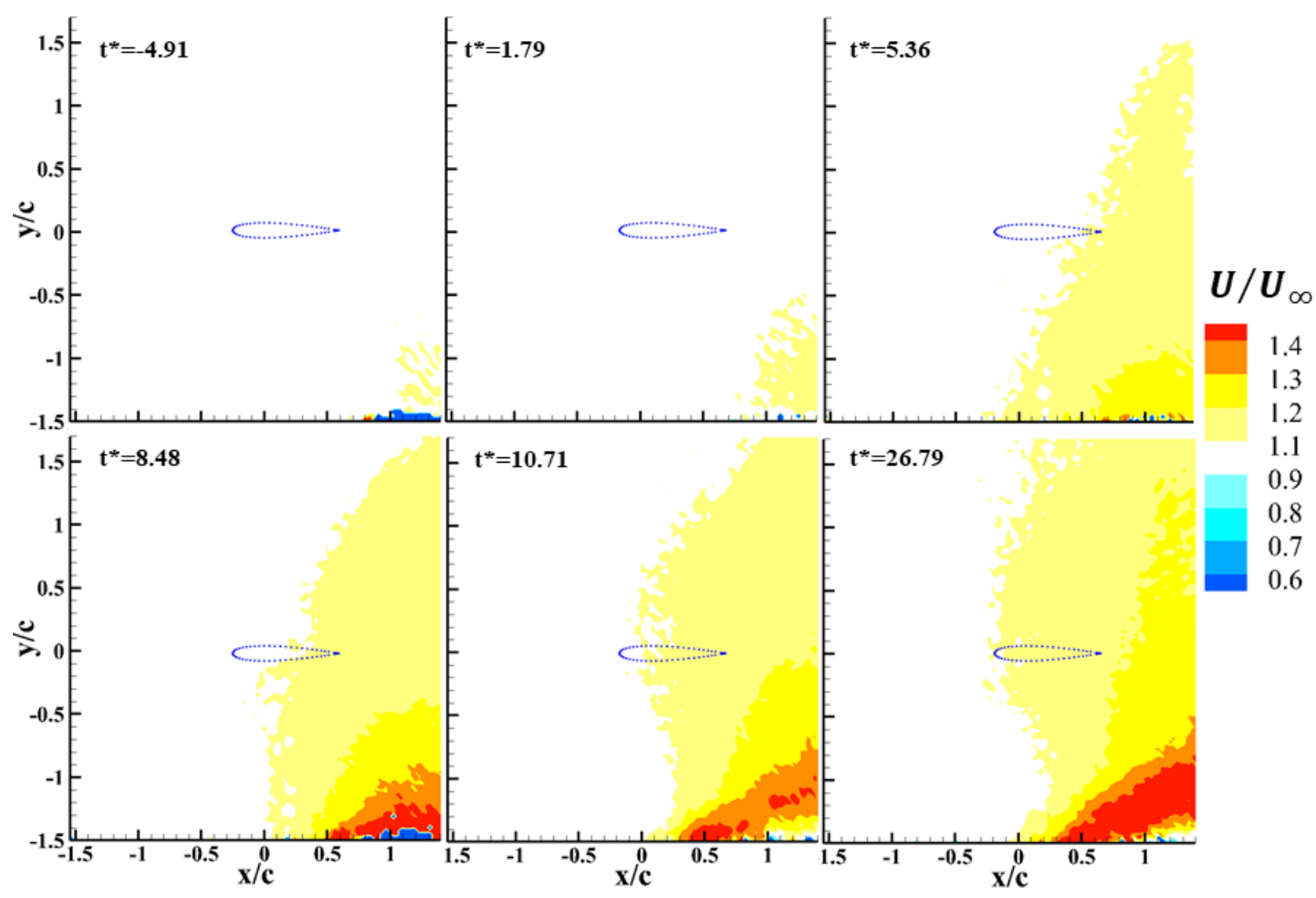

Figure 134: Mid power gust actuation. $U / U_{\infty}$.

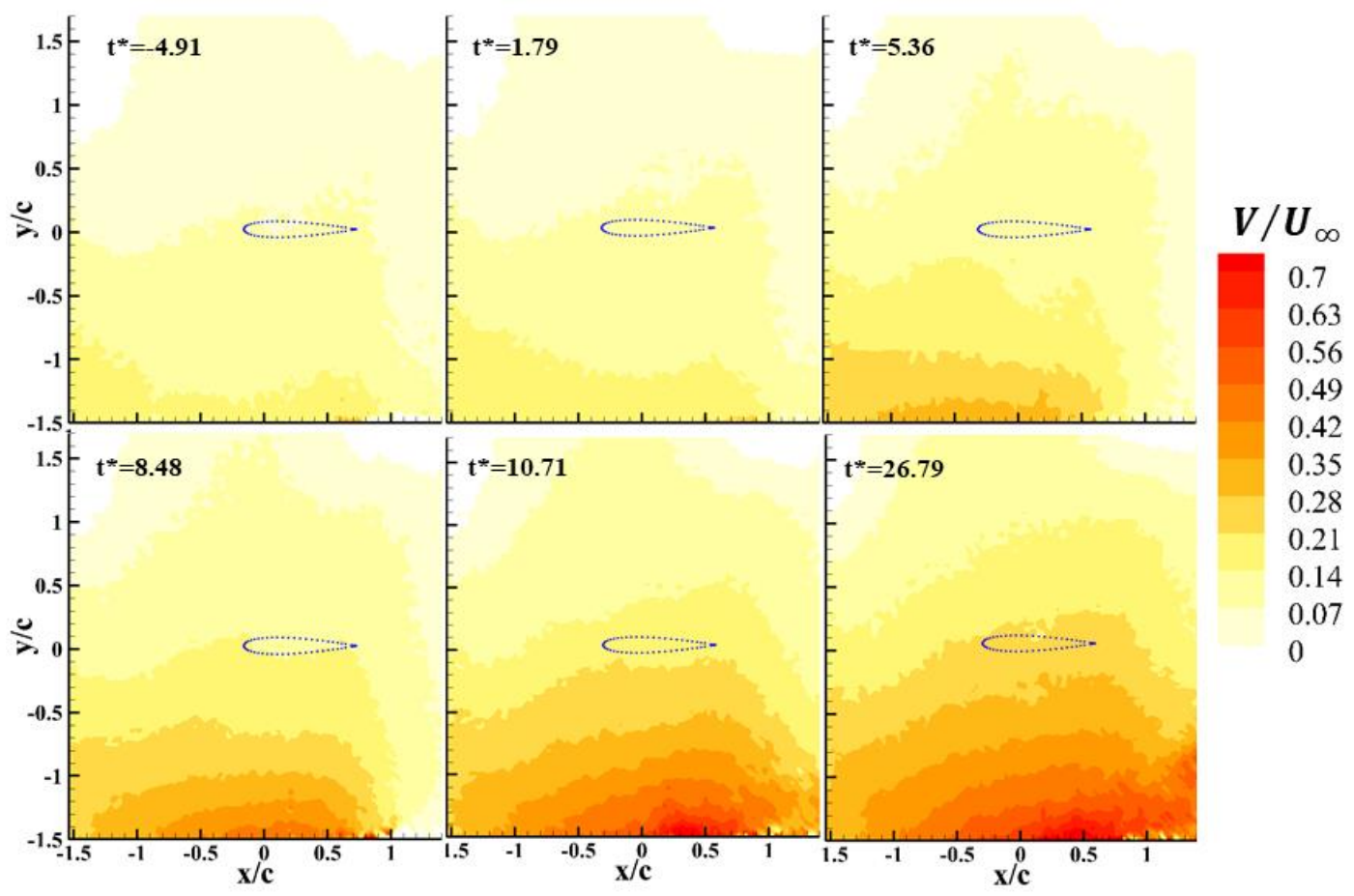

Figure 135: Mid power gust auction. Gust ratio. 


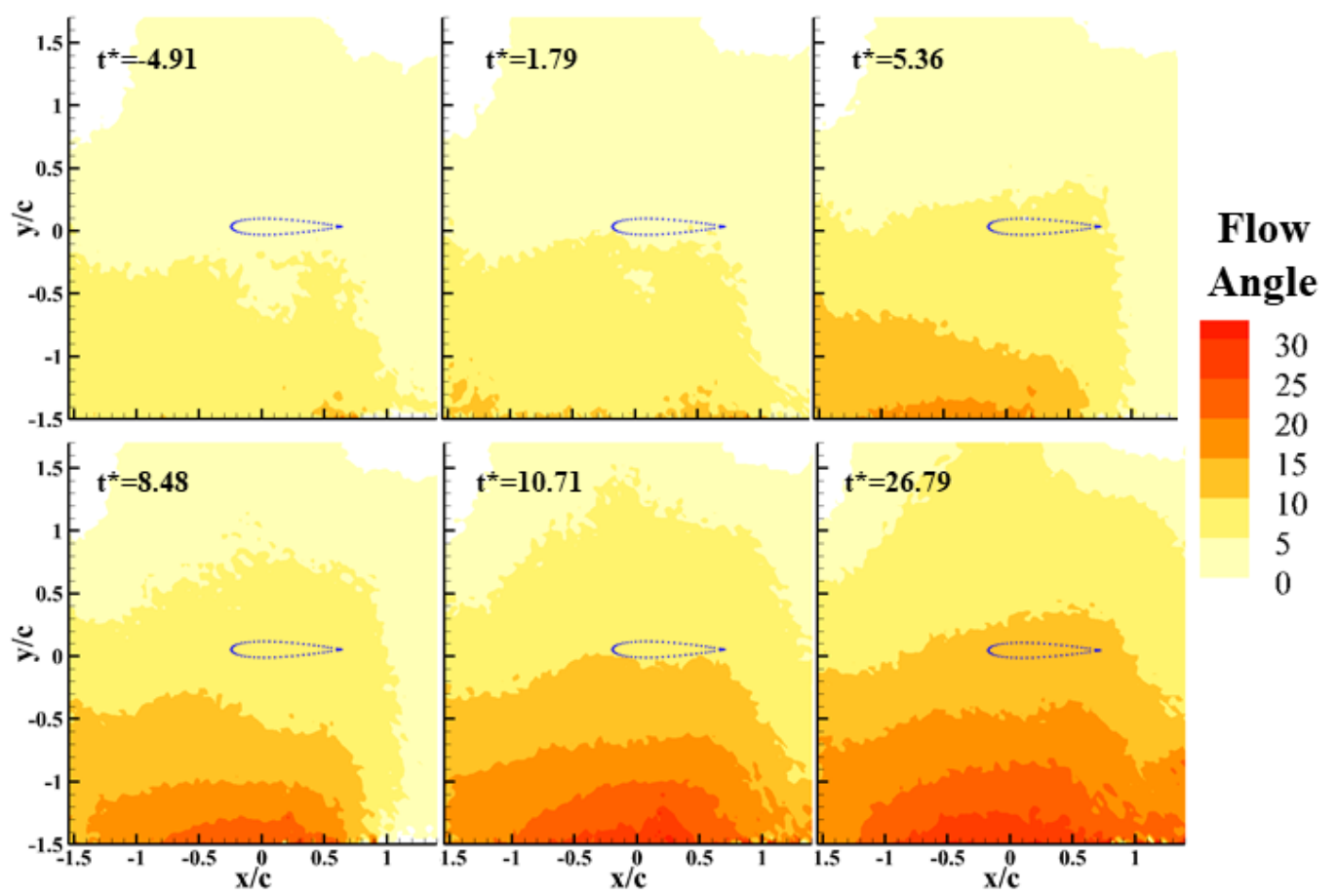

Figure 136: Mid power gust actuation. Flow angle.

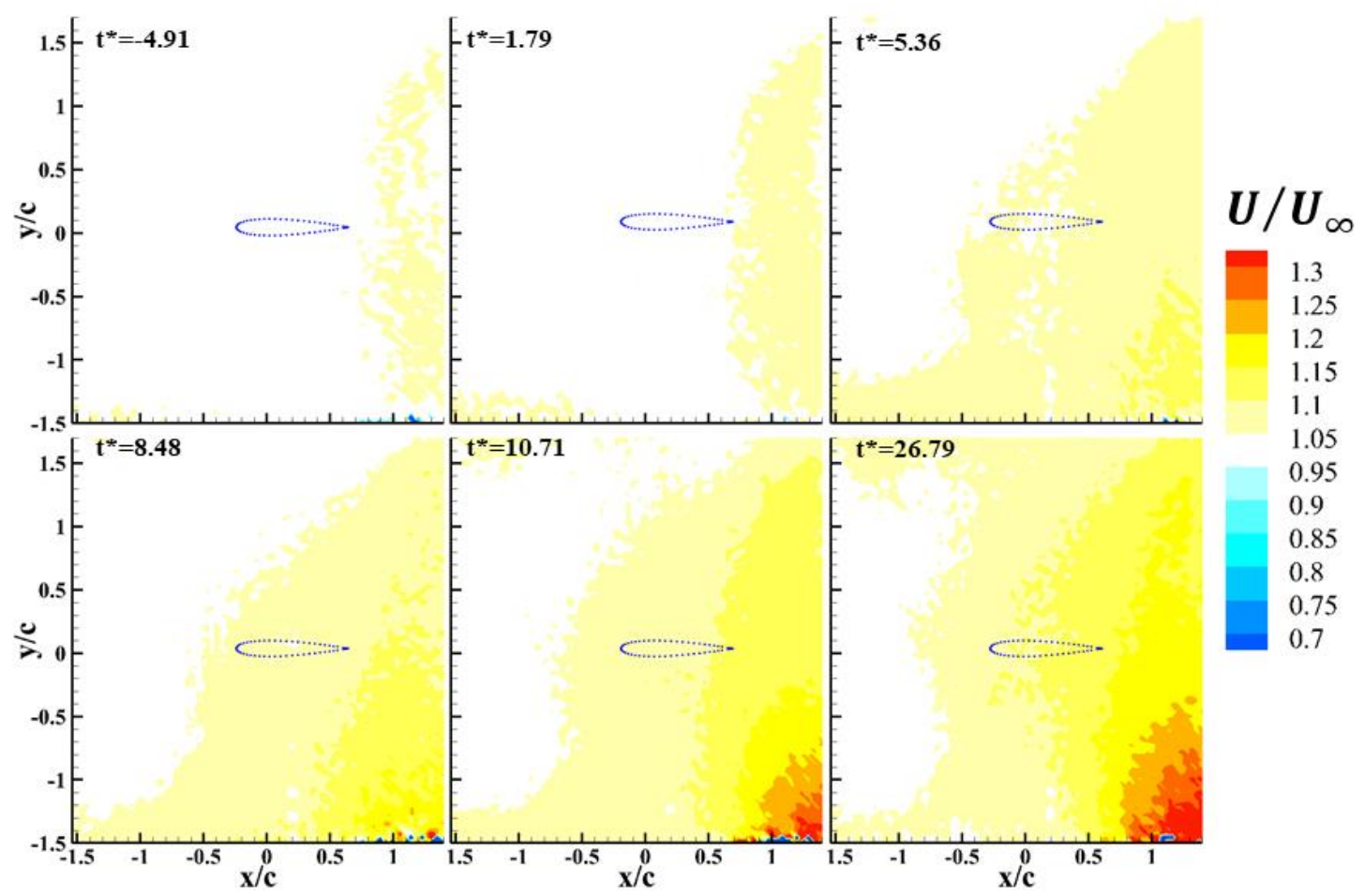

Figure 137: Low power gust actuation. $U / U_{\infty}$. 


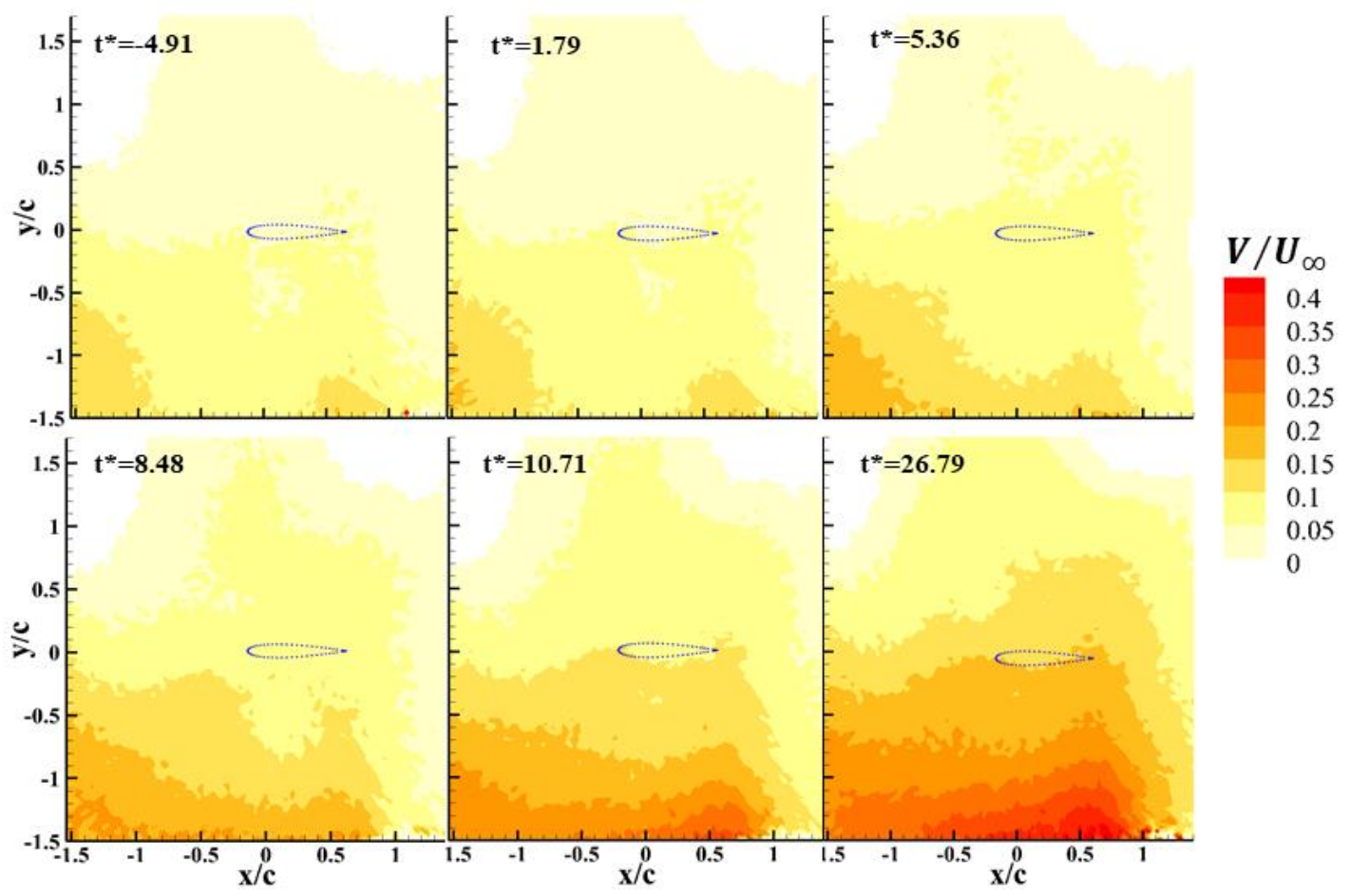

Figure 138: Low power gust actuation. Gust ratio.

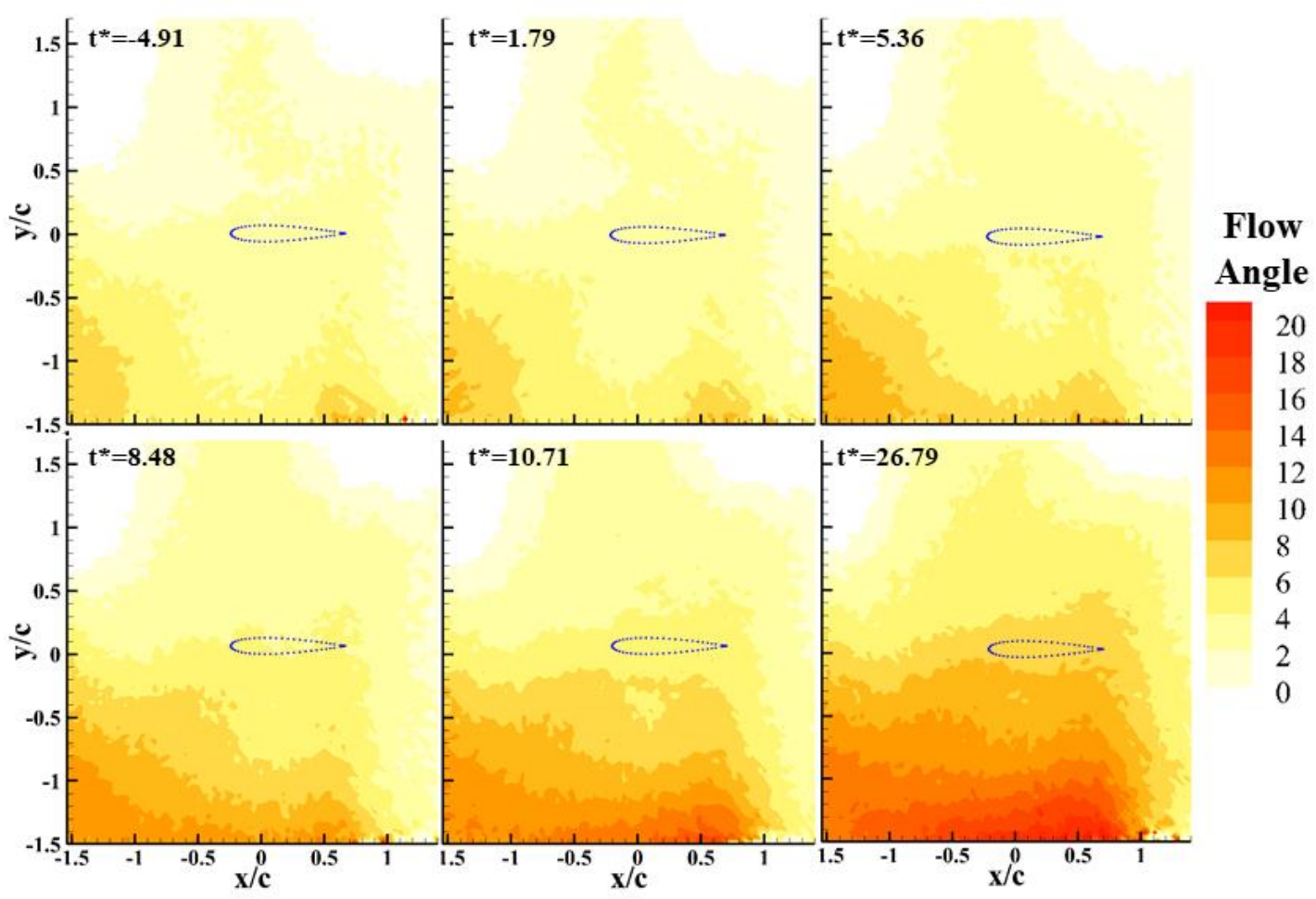

Figure 139: Low power gust actuation. Flow angle. 


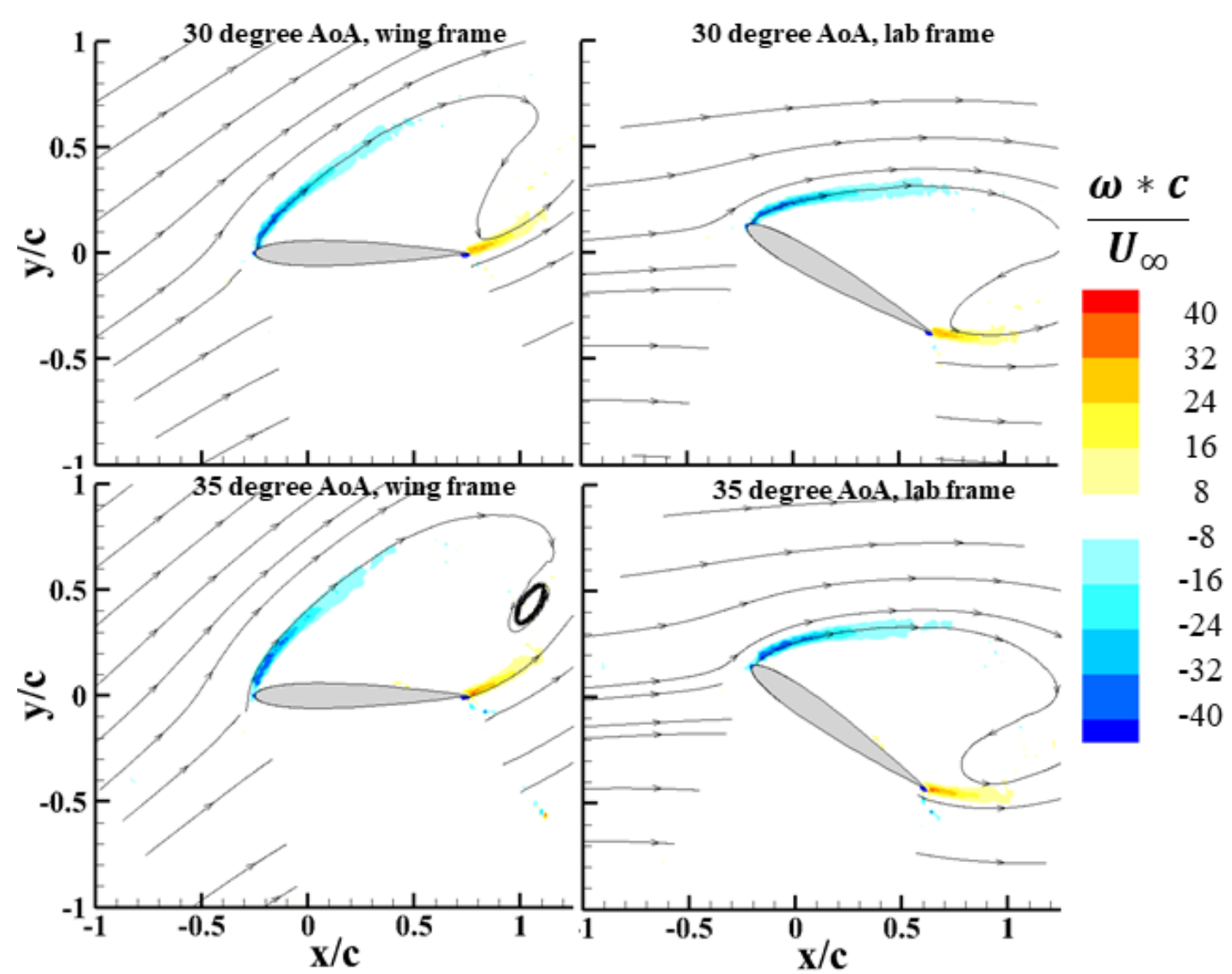

Figure 140: High wing angles for a static wing, both lab and wing reference frames shown.

The next set of images presented are with the wing at an estimated 5-degree effective angle of attack. They can be seen in Figure 141. 


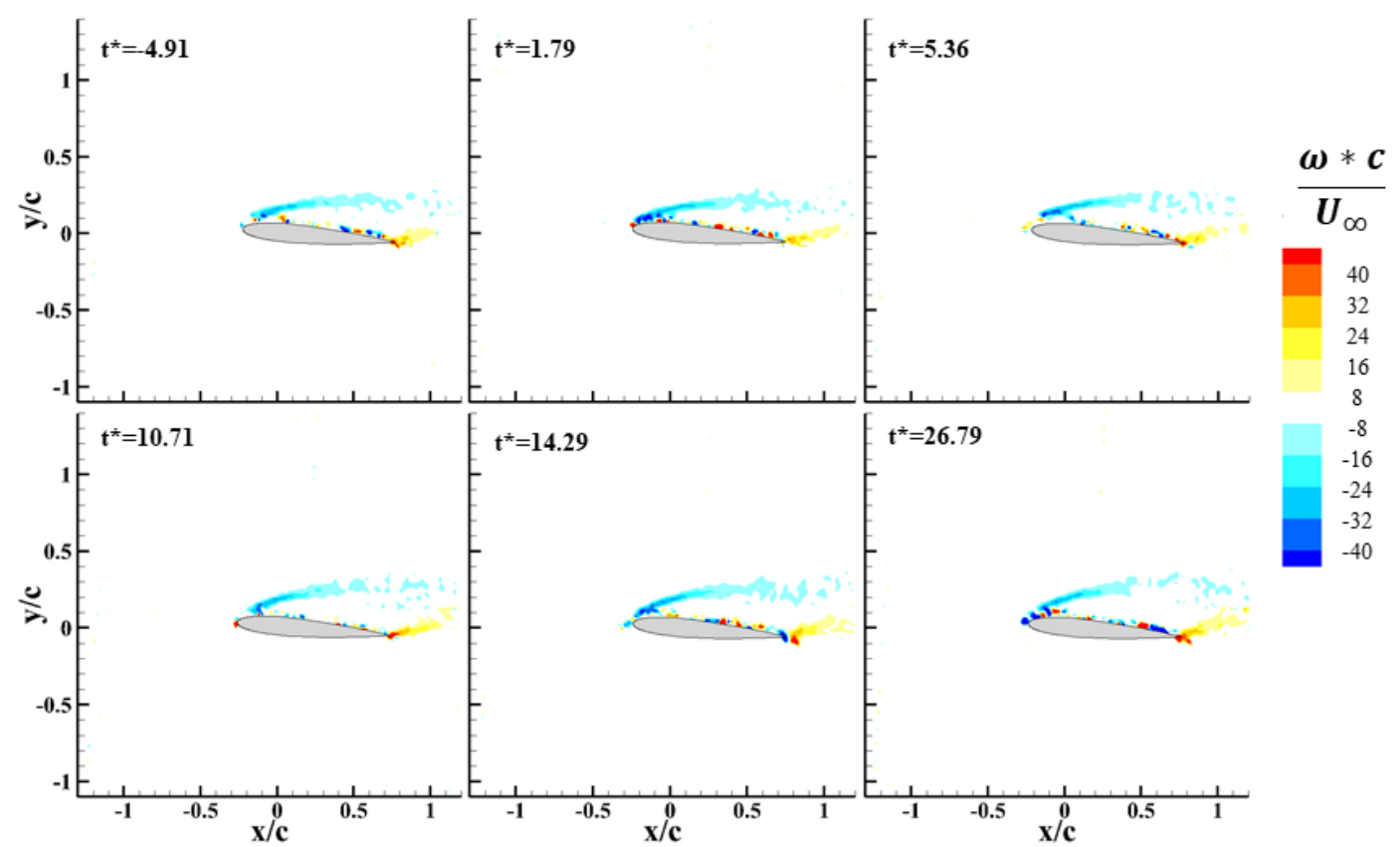

Figure 141: Actuating mid power gust; wing at 5-degree initial effective angle.

There is an immediate issue when viewing these images. It was known from previous tests, that the wing should not stall until after 5 degrees. However, the initial frames of this gust actuation case show the wing already experiencing stalled conditions. This means that the determination of the angle at which to place the wing to create an initial 5-degree effective angle of attack was incorrect and off by about 3 to 4 degrees. It is unsure how this mistake occurred; however, this highlights the difficulty in predicting the strength a gust will have after interacting with a wing. Clearly the prediction from the wing-less gust actuation case was not correct in this instant. Therefore, comparisons to static wings were not created as they were sure to be skewed from the actual data.

The final image sets come with the wing at what was assumed to be a 10 -degree initial effective angle of attack. They can be seen in Figure 142. 


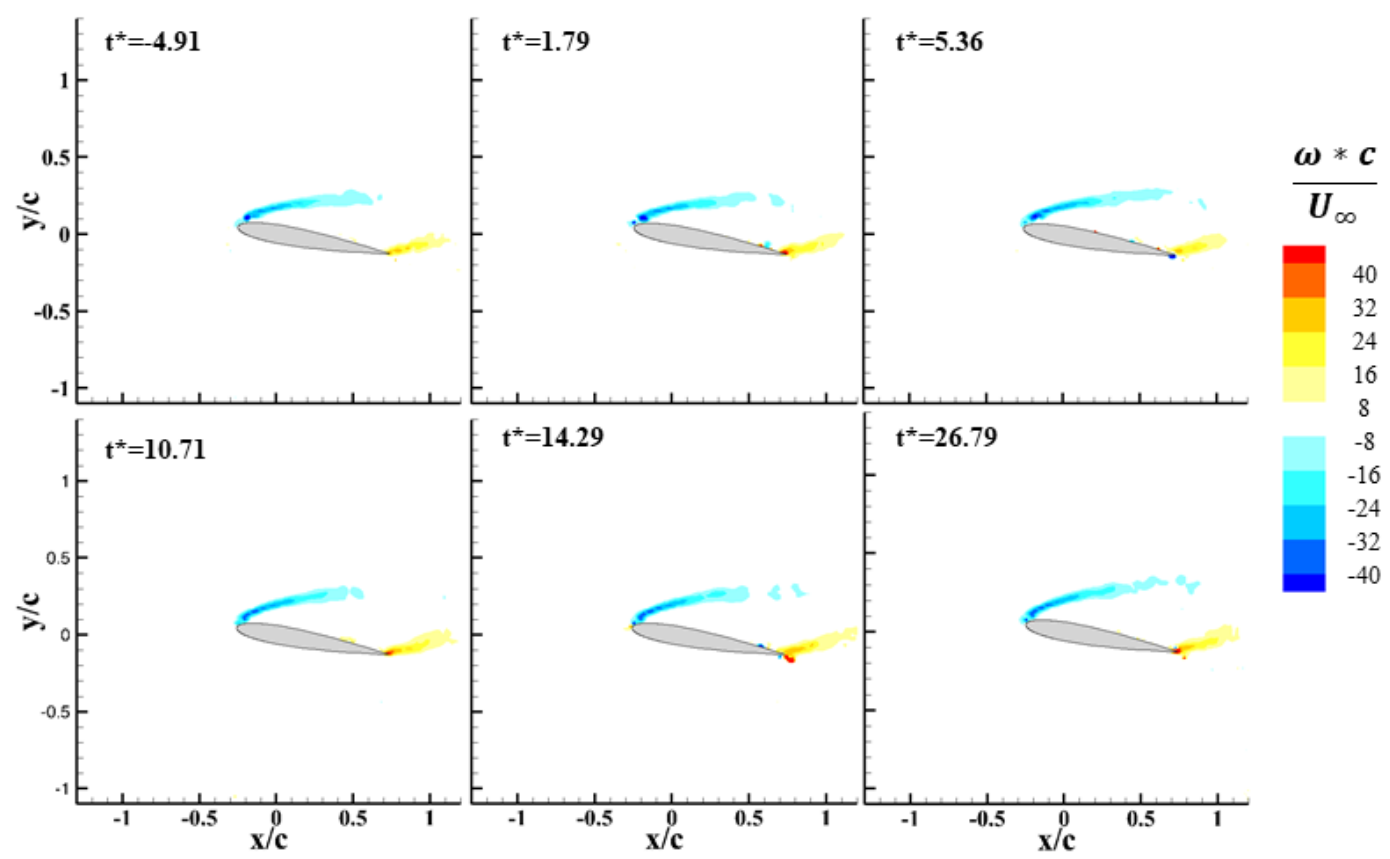

Figure 142: Actuating mid power gust; wing at 10-degree initial effective angle.

There is again some issue in these frames with the initial effective angle calculation. As before, the wing seems to be pitched too far and is creating an even higher initial effective angle than desired. Thus again, comparisons were not made as they would hold no scientific meaning. 


\section{Bibliography}

[1] T. J. Mueller, "Overview," in Fixed and Flapping Wing Aerodynamics for Micro Air Vehicle Applications, American Institute of Aeronautics and Astronautics, Inc., 2001, p. 3.

[2] "Small UAS," AeroVironment, [Online]. Available: https://www.avinc.com/innovative-solutions/small-uas. [Accessed 22 January 2018].

[3] "PD-100 PRS," FLIR, [Online]. Available: http://www.proxdynamics.com/products/pd-100-black-hornet-prs. [Accessed 22 January 2018].

[4] "AeroVironment, Developer of the Nano Hummingbird, Unveils Snipe ${ }^{\mathrm{TM}}$, A New, Stealthy Nano Quadrotor UAS," AeroVironment, 9 May 2017. [Online]. Available: https://www.avinc.com/resources/view/pressreleases/aerovironment-developer-of-the-nano-hummingbird-unveils-snipe-anew-stealth. [Accessed 22 January 2018].

[5] "Honeywell T-Hawk Micro Air Vehicle (MAV)," Army Technology, [Online]. Available: http://www.army-technology.com/projects/honeywell-thawk-mavus-army/. [Accessed 6 March 2018].

[6] J. Excell, "Insect Inspiration: UK Defence Drone Mimics Dragonfly Flight," The Engineer, 16 November 2016. [Online]. Available: https://www.theengineer.co.uk/insect-inspiration-uk-defence-drone-mimicsdragonfly-flight/. [Accessed 6 February 2018].

[7] "The Delfly Project," Delft University, [Online]. Available: http://www.delfly.nl/. [Accessed 22 January 2018].

[8] J. M. McMichael and M. S. Francis, "Micro Air Vehicles- Towards a New Dimension in Flight," DARPA Report, 1997.

[9] M. E. Dempsey, "U.S. Army Unmanned Aircraft Systems Roadmap 20102035," U.S. Army Report, 2010.

[10] K. Viswanath and D. K. Tafti, "Effect of Frontal Gusts on Forward Flapping Flight," AIAA Journal, vol. 48, no. 9, 2010.

[11] G. Bohrer, D. Brandes, M. T. James, K. L. Bildstein, T. A. Miller, M. Lanzone, T. Katzner, C. Maisonneuve and J. A. Tremblay, "Estimating Updraft Velocity Components Over Large Spatial Scales: Contrasting Migration Strategies of Golden Eagles and Turkey Vulture," Ecology Letter, vol. 15, no. 2, pp. 96-103, 2012.

[12] W. C. Conover and C. J. Wentzien, "Winds and Temperature to Forty Kilometers," Journal of Meteorology, vol. 12, pp. 160-164, 1954.

[13] S. Watkins, J. Milbank and B. J. Loxton, "Atmospheric Winds and Their Implications for Microair Vehicles," AIAA Journal, vol. 44, no. 11, 2006.

[14] R. C. Nelson, Flight Stability and Automatic Control, WCB McGraw-Hill, 1998. 
[15] U. Saxena and I. A. Faruque, "Gramian-Inspired Unmanned Aerial Flight Control for Atmospheric Energy Capture," in AIAA Atmospheric Flight Mechanics Conference, AIAA AVIATION Forum, (AIAA 2016-3712), Washington, D.C., 2016.

[16] B. G. Newman, "Soaring and Gliding FLight of the Black Vulture," Journal of Experimental Biology, vol. 35, no. 2, pp. 280-285, 1957.

[17] V. A. Tucker, "Gliding Birds: The Effect of Variable Wing Span," Journal of Experimental Biology, vol. 133, pp. 33-58, 1987.

[18] D. Lentink, U. K. Müller, E. J. Stamhuis, R. d. Kat, W. v. Gestel, L. L. M. Veldhuis, P. Henningsson, A. Hedenstrom, J. J. Videler and J. L. v. Leeuwen, "How swifts control their glide performance with morphing wings," Nature, vol. 446, pp. 1082-1085, 26 April 2007.

[19] H. Küssner, "Stresses Produced in Airplane Wings by Gusts," NACA Technical Memorandums, 1932.

[20] H. N. Murrow, K. G. Pratt and J. C. Houbolt, "NACA/NASA Research Related to Evolutions of U.S. Gust Design Criteria," in 30th Structures, Structural Dynamics and Materials Conference, Structures, Structural Dynamics, and Materials and Co-located Conferences, (1989-1373), Mobile, AL, 1989.

[21] T. L. Jordan, J. V. Foster, R. M. bailey and C. M. Belcastro, "AirSTAR: A UAV Platform for Flight Dynamics and Control System Testing," in 25th AIAA Aerodynamic Measurement Technology and Ground Testing Conference, Fluid Dynamics and Co-located Conferences, (AIAA 2006-3307), San Francisco, CA, 2006.

[22] M. Usui, A. Simpson, S. Smith and J. Jacob, "Development and Flight Testing of UAV with Inflatable-Rigidizable Wings," in 42nd AIAA Aerospace Sciences Meeting and Exhibit, Aerospace Sciences Meetings, (AIAA 2004-1373), Reno, NV, 2004.

[23] "TM1000 DSMX Full-Range Aircraft Telemtry," Spektrum, [Online]. Available: https://www.spektrumrc.com/Products/Default.aspx?ProdID=SPM9548. [Accessed 23 January 2018].

[24] J. P. How, B. Bethke, A. Frank, D. Dale and J. Vian, "Real-Time Indoor Autonomous Vehicle Test Environment," IEEE, vol. 28, no. 2, pp. 51-64, 2008.

[25] S. Zarovy, M. Costello, A. Mehta, G. Gremillion, D. Miller, B. Ranganathan, J. S. Humbert and P. Samuel, "Experimental Study of Gust Effects on Micro Air Vehicles," in AIAA Atmospheric Flight Mechanics Conference, Guidance, Navigation, and Control and Co-located Conferences, (AIAA 2010-7818), Toronto, On, 2010.

[26] R. R. Meyer, Jr., "A Unique Flight Test Facility: Description and Results," NASA, 1982.

[27] B. L. Stevens, F. L. Lewis and E. N. Johnson, Aircraft Control and Simulation, Hoboken: John Wiley \& Sons, Inc., 2016. 
[28] A. Sivakumar and C. K.-Y. Tan, "Formation Control for Lightweight UAVs Under Realistic Communications and Wind Conditions," in AIAA Guidance, Navigation, and Control Conference, Guidance, Navigation, and Control and Co-located Conferences, (AIAA 2009-5885), Chicago, Il, 2009.

[29] "Extend It," X Plane, [Online]. Available: http://www.xplane.com/desktop/extend-it/. [Accessed 6 February 2018].

[30] A. M. Murch, Y. C. Paw, R. Pandita, Z. Li and G. J. Balas, "A Low Cost Small UAV Flight Research Facility," 1st CESA Specialist Conference on Guidance, Navigation, and Control, pp. 29-40, 2011.

[31] V. Gavrilets, I. Martinos, B. Mettler and E. Feron, "Flight Test and Simulation Results for an Autonomous Aerobatic Helicopter," in IEEE, Digital Avionics Systems Conference, 2002. Proceedings. The 21st, 2002.

[32] R. V. Rhode and E. E. Lundquist, "Preliminary Study of Applied Load Factors in Bumpy Air," NACA-TN-374, Washington Dc, 1931.

[33] P. Donely, "An Experimental Investigation of the Normal Acceleration of an Airplane Model in a Gust," NACA-TN-595, Washington DC, 1939.

[34] T. D. Reisert, "Tests of a 1/17-scale Model of the XBDR-1 Airplane in the NACA Gust Tunnel," NACA, Washington DC, 1944.

[35] D. J. Maglieri and T. D. Reisert, "Gust-Tunnel Investigation of the Effect of a Sharp-Edge Gust on the Flapwise Blade Bending Moments of a Model Helicopter Rotor," NACA TN 3470, Washington DC, 1955.

[36] G. K. Hunt, D. R. Roberts and D. Walker, "Measurments of Transient Pressures on a Narrow-Delta Wing due to an Upward Gust," Ministry of Aviation, London, 1964.

[37] D. W. Holmes, "Lift and Measurments in an Aerofoil in Unsteady Flow," The American Society of Mechanical Engineers, 1973.

[38] M. H. Patel and G. J. Hancock, "A Gust Tunnel Facility," Procurment Executive Minstry of Defense, London, 1977.

[39] L. C. Garby, A. M. Kuethe and J. D. Schetzer, "The Generation of Gusts in a Wind Tunnel and Measurement of Unsteady Lift on an Airfoil," University of Michigan, Ann Arbor, 1957.

[40] A. M. Kuethe and J. D. Shetzer, "The Generation of Gusts in a Wind Tunnel and Measurment of Unsteady Lift on an Airfoil," The University of Michigan, Ann Arbor, 1957.

[41] R. J. Hakkinen and A. S. J. Richardson, "Theoretical and Experimental Investigation of Random Gust Loads. Part I- Aerodynamic Transfer Function of a Simple Wing Configuration in Incompressible Flow," NACA-TN-3878, Washington, 1957.

[42] E. A. L. Hufstedler, "Experimental Generation and Modeling of Vortical Gusts and Their Interactions with an Airfoil," PHD of Aeronautics Engineer, Caltech, Pasadena, CA, 2017. 
[43] H. Biler, "Gust Chareacterization of Flapping Wing Applications," Bachelors of Science, Aeronautical Engineering, Istanbul Technical University, Istanbul, 2015.

[44] J. M. Roadman and K. Mohseni, "Gust Characterization and Generation for Wind Tunnel Testing of Micro Aerial Vehicles," in 47th AIAA Aerospace Sciences Meeting including The New Horizons Forum and Aerospace Exposition, Aerospace Sciences Meetings, (AIAA 2009-1290), Orlando, FL, 2009.

[45] C. F. Reid and C. G. J. Wrestler, "An Investigation of a Device to Oscillate a Wind-Tunnel Airstream," NASA, Washington, 1961.

[46] J. J. Gilman and R. M. Bennett, "A Wind-tunnel Technique for Measuring Frequency-Response Functions for Gust Load Analyses," Journal of Aircraft, vol. 3, no. 6, pp. 535-540, 1966.

[47] D. A. Buell, "An Experimental Investigation of the Velocity Fluctuations Behind Oscillating Vanes," NASA-TN-D-5543, Washinton DC, 1969.

[48] R. M. Umbarger, "Wind Tunnel Gust Simulation," Master of Science Engineering, Princeton University, Princeton, NJ, 1970.

[49] N. D. Ham, P. H. Bauer and T. L. Lawrence, "Wind Tunnel Generation of Sinusiodal Lateral and Longitudinal Gust by Circulation Control of Twin Parellel Airfoils," NASA-CR-137547, ASRL-TR-174-3, Washington DC, 1974.

[50] D. M. Tang, P. G. A. Cizmas and E. H. Dowell, "Experiments and Analysis for a Gust Generator in a Wind Tunnel," Journal of Aircraft, vol. 33, no. 1, pp. 139148, 1996.

[51] H. Stapountzis, "An Oscillating Rig for the Generation of Sinusoidal Flows," Jounral of Physics E: Scientific Instruments, vol. 15, pp. 1173-1176, 1982.

[52] V. Brion, A. Lepage, Y. Amosse, D. Soulevant, P. Senecat and J. C. Abart, "Generation of Vertical Gusts in a Transonic Wind Tunnel," Experiments in Fluids, vol. 56, no. 145, 2016.

[53] P. M. G. J. Lancelot, J. Sodja, N. P. M. Werter and R. De Breuker, "Design and Testing of a Low Subsonic Wind Tunnel Gust Generator," 16th International Forum on Aeroelsticity and Structural Dynamics, 2015.

[54] L. Yi, X. Changchuan, Y. Chao and C. Jialin, "Gust Response Analysis and Wind Tunnel Test for a High-Aspect Ratio Wing," Chinese Journal of Aeronautics, vol. 29, no. 1, pp. 91-103, 2016.

[55] D. Kubo, "Gust Response Evaluation of Small UAS via Free-Flight in Gust Wind Tunnel," in AIAA Atmospheric Flight Mechanics Conference, AIAA SciTech Forum, (AIAA 2018-0297), Orlando, FL, 2018.

[56] J. Bicknell and A. G. Parker, "A Wind-Tunnel Stream Oscillation Apparatus," Journal of Aircraft, vol. 9, no. 6, pp. 446-447, 1972.

[57] H. Kobayashi and A. Hatanaka, "Active Generation of Wind Gust in a TwoDimensional Wind Tunnel," Journal of Wind Engineering and Industrial Aerodynamics, vol. 41, no. 1-3, pp. 959-970, 1992. 
[58] D. L. Grissom and W. J. Devenport, "Development and Testing of a Deterministic Disturbance Generator," in 10th AIAA/CEAS Aeroacoustics Conference, Aeroacoustics Conferences, (AIAA 2004-2956), 2004.

[59] F. L. J. Haan and P. P. Sarkar, "Development of an Active Gust Generation Mechanism on a Wind Tunnel for Wind Engineering and Industrial Aerodynamics Applications," Wind and Structures, vol. 9, no. 5, pp. 369-386, 2006.

[60] S. Ricci and A. Scotti, "Wind Tunnel Testing of an Active Controlled Wing under Gust Excitation," in 9th AIAA/ASME/ASCE/AHS/ASC Structures, Structural Dynamics, and Materials Conference (AIAA 2008-1727), Schaumburg, IL, 2008.

[61] H. Mai, J. Neumann and H. Hennings, "Gust Response: A Validation Experiment and Preliminary Numerical Simulations," IFASD, vol. 51, 2011.

[62] A. J. Saddington, M. V. Finnis and K. Knowles, "The Characterisation of a Gust Generator for Aerodynamics Testing," Journal of Aerospace Engineering, 2014.

[63] K. Granlund, B. Monnier, M. Ol and D. Williams, "Airfoil Longitudinal Gust Response in Seperated vs. Attached Flows," Physics of Fluids, vol. 26, no. 2, 2014.

[64] F. O. Carta, "A Comparison of the Pitching and Plunging Response of an Oscillating Airfoil," NASA-CR-3172, 1979.

[65] S. Sunada, K. Kawachi, A. Matsumoto and A. Sakaguchi, "Unsteady Forces on a Two-Dimensional Wing in Plunging and Pitching Motions," AIAA Journal, vol. 39, no. 7, pp. 1230-1239, 2001.

[66] W. Shyy, Y. Lian, J. Tang, D. Viieru and H. Liu, "Aerodynamics of Low Reynolds Number Flyers," Cambridge University Press, 2008.

[67] D. Quinn, D. Kress, A. Stein, M. Wegrzynski, L. Hamzah and D. Lentink, "How birds can negate gusts and maintain heading by crabbing into the wind passively," in 70th Annual Meeting of the APS Division of Fluid Dynamics, Denver, 2017.

[68] W. Shyy, Y. Lian, J. Tang, H. Liu, P. Trizila, B. Stanford, L. Bernal, C. Cesnik, P. Friedmann and P. Ifju, "Computational Aerodynamics of Low Reynolds Number Plunging, Pitching and Flexible Wings for MAV Applications," in AIAA Aerospace SSciences Meeting and Exhibit (AIAA 2008-523), Reno, NV, 2008.

[69] R. Wokoeck and R. Radespiel, "Experimental and Numerical Investigations of Pitch-Plunging Wing Aerodynamics at Low Reynolds Number," International Journal of Engineering Systems Modelling and Simulation, vol. 5, pp. 19-32, 2013.

[70] Y. S. Baik, L. P. Bernal, K. Granlund and M. V. Ol, "Unsteady force Generation and Vortex Dynamics of Pitching and Plunging Aerofoils," Journal of Fluid Mechancis, vol. 709, pp. 37-68, 2012. 
[71] G. Perrotta and A. R. Jones, "Transient Aerodynamics of Large Transverse Gusts and Geometrically Similar Maneuvers," in 54th AIAA Aerospace Sciences Meeting, AIAA SciTech Forum, (AIAA 2016-2074), San Diego, CA, 2016.

[72] P. R. R. J. Stevens and H. Babinsky, "Experiments to Investigate Lift Production Mechanisms on Pitching Flat Plates," Experiments in Fluids, vol. 58, no. 7, 2016.

[73] G. Perrotta and A. Jones, "Unsteady Forcing on a Flat-Plate Wing in Large Transverse Gusts," Experiments in Fluids, vol. 58, no. 101, 2017.

[74] S. Corkery, H. Babinsky and J. Harvey, "Response of a Flat Plate Wing to Transverse Gust at Low Reynolds Numbers," in 2018 AIAA Aerospace Sciences Meeting, AIAA SciTech Forum, (AIAA 2018-1082), Orlando, FL, 2018.

[75] H. Biler, C. Badrya and A. R. Jones, "Experimental and Computational Investigation of Transverse Gust Encounters," in 2018 AIAA Aerospace Sciences Meeting, AIAA SciTech Forum, (AIAA 2018-0571), Orlando, FL, 2018.

[76] V. V. Golubev and M. R. Visbal, "Modeling MAV Response in Gusty Urban Enviroment," International Journal of Micro Air Vehicles, vol. 4, no. 1, pp. 7992, 2012.

[77] R. E. Gordnier and M. R. Visbal, "Implicit LES Computation of a VorticalGust/Wing Interaction for Transitional Flow," in 53rd AIAA Aerospace Sciences Meeting, AIAA SciTech Forum, (AIAA 2015-1067), Kissimmee, FL, 2015.

[78] A. Zaide and D. Raveh, "Numerical Simulation and Reduced-Order Modeling of Airfoil Gust Response," AIAA Jounral, vol. 44, no. 8, 2006.

[79] G. Yang and S. Obayashi, "Numerical Analyses of Discrete Gust Response for an Aircraft," Journal of Aircraft, vol. 41, no. 6, 2004.

[80] E. H. Hirschel, H. Prem and G. Madelung, Aeronautical Research in Germany: From Lilienthal until Today, Berlin: Springer, 2004.

[81] T. Theodore, "General Theory of Aerodynamic Instability and the Mechanism of Flutter," NACA Report 496, Washington DC, 1935.

[82] C. White, E. W. Lim, S. Watkins, A. Mohamed and M. Thompson, "A feasability Study of Micro Air Vehicles Soaring Tall Buildings," Jounral of Wind Engineering and Industrial Aerodynamics, vol. 103, pp. 41-49, 2012.

[83] "DMC-40x0," Galil, [Online]. Available: http://www.galilmc.com/motioncontrollers/multi-axis/dmc-40x0. [Accessed 82 2018].

[84] A. K. Prasad, "Particle Image Velocimetry," Current Science, vol. 79, no. 1, pp. 51-60, 2000.

[85] R. J. Adrian and J. Westerweel, Particle Image Velocimetry, New York: Cambridge University Press, 2011.

[86] L. M. Lourenco, A. Krothapalli and C. A. Smith, Particle Image Velocimetry, Berlin: Springer, 1989.

[87] R. K. Cohn and M. M. Koochesfahani, "The Accuracy of Remapping Irreguarly Spaced Velocity Data onto a Regular Grid and the Computation of Vorticity," Experiments in Fluid, vol. 29, no. Supplement 1, p. S061-S069, 2000. 
[88] R. J. Margason, "The Path of a Jet Directed at Large Angles to a Subsonic Free Stream," NASA-TN-D-4919, Washington DC, 1968.

[89] J. H. W. Lee and P. Neville-Jones, "Initial Dilution of Horizontal Jet in Crossflow," Journal of Hydraulic Engineering, vol. 113, no. 5, pp. 615-629, 1987.

[90] A. Krothapalli, L. Lourenco and J. M. Buchlin, "Seperated Flow Upstream of a Jet in a Crossflow," AIAA Journal, vol. 28, no. 3, pp. 414-420, 1986.

[91] N. M. Shumway, M. Gabryszuk and S. J. Laurence, "Flapping Tandem-Wing Aerodynamics: Dragonflies in Steady Foward Flight," in 2018 AIAA Aerospace Sciences Meeting, AIAA SciTech Forum, (AIAA 2018-1290), Orlando, FL, 2018. 